\title{
DEVELOPMENT OF AN INTRAVASCULAR DOPPLER OPTICAL COHERENCE TOMOGRAPHY IMAGING TECHNIQUE FOR NEUROVASCULAR APPLICATIONS
}

\author{
by \\ Barry Vuong \\ Bachelor of Engineering, Computer Engineering, Ryerson, 2007 \\ Master of Applied Science, Electrical \& Computer Engineering, Ryerson, 2009 \\ A dissertation \\ presented to Ryerson University \\ in partial fulfillment of the \\ requirements for the degree of \\ Doctor of Philosophy \\ in the Program of \\ Electrical \& Computer Engineering \\ Toronto, Ontario, Canada, 2015 \\ (C)Barry Vuong 2015
}




\section{AUTHOR'S DECLARATION FOR ELECTRONIC SUBMISSION OF A DISSERTATION}

I hereby declare that I am the sole author of this dissertation. This is a true copy of the dissertation, including any required final revisions, as accepted by my examiners.

I authorize Ryerson University to lend this dissertation to other institutions or individuals for the purpose of scholarly research.

I further authorize Ryerson University to reproduce this dissertation by photocopying or by other means, in total or in part, at the request of other institutions or individuals for the purpose of scholarly research.

I understand that my dissertation may be made electronically available to the public. 
Development of an intravascular Doppler optical coherence tomography imaging technique for neurovascular applications Doctor of Philosophy 2015

Barry Vuong

Electrical \& Computer Engineering

Ryerson University

\begin{abstract}
The rupture of an intracranial aneurysm can cause spontaneous subarachnoid hemorrhage and result in sudden death. A large portion of intracranial aneurysms occurs near the center of the head, at the skull base, which poses significant technical challenge to neurosurgeons due to limited accessibility. The utilization of angiography is prominent during the treatment of intracranial aneurysms. However, malapposition of stent or incomplete packing of the intracranial aneurysm can be difficult to assess with angiography, and could lead to severe postoperative complications. As a result, angiography may not be sufficient in determining the risk of rupture as the compensatory mechanisms are known to occur at the microstructural level due to the local hemodynamics in the arterial lumen, as well as in evaluating the intraoperative treatment.

In this work, we describe a method for assessing intracranial aneurysm through the evaluation of blood flow within the lumen and morphological structures of the arterial wall with optical coherence tomography (OCT). Sterile intravascular fiber-optic catheters can be introduced in the artery to detect blood flow. Prior to this work, limited investigations of catheter based Doppler OCT (DOCT) were reported. A novel signal processing technique was developed to further reduce the effect of Doppler noise within a catheter based DOCT system. This technique consisted of splitting the interferogram of an OCT signal prior to estimating the Doppler shift. This split spectrum DOCT (ssDOCT) method was evaluated through flow models and porcine models, as well as through the correlation between ssDOCT algorithm
\end{abstract}


and computational fluid dynamic (CFD) models. It was observed that ssDOCT provided improved Doppler artefact suppression over the conventional DOCT technique. ssDOCT also provided the ability to estimate lower velocities within the DOCT image to measure the hemodynamic patterns around stent struts in both the internal carotid and patient specific flow phantoms. An OCT imaging study was also conducted consisting of surgically resected human intracranial aneurysms. Further enhancement of the detection of these key morphological structures was demonstrated by an optical-attenuation imaging variant of OCT. The presented techniques could provide further insights to the cause of intracranial aneurysm rupture and vascular healing mechanisms. 


\section{Acknowledgements}

One of the great joys of compiling my $\mathrm{PhD}$ research is to reflect over the journey that I have took and remember all the friends and family who have supported me throughout the years. First and foremost I would like to express my heartfelt gratitude to both my supervisors, Dr. Victor X.D. Yang and Dr. Michael C. Kolios for not only accepting me into their lab, but also being great mentors and role models.

I first met Dr. Michael C. Kolios during my first research assistantship in the summer of 2006. I remember he would be (and still is) excited and full of energy over every new data that came from the lab. This would resonate across to the seating area where I was working and how I view research. Over the course of my $\mathrm{PhD}$, Dr. Kolios would always take the time to meet with me to discuss about my projects and ensure that I don't deviate far from the apple tree.

During my masters I had sat in Dr. Victor X.D. Yang's biophotonics graduate course. The enthusiasm and inspiration he displayed in class was what drew me into my $\mathrm{PhD}$. Since then, I have not regretted this decision. Victor has opened countless opportunities for my career throughout my PhD. It began with encouraging me to join a national photonics student group, where I had become president shortly after. Then he presented me with the opportunity to send me to Massachusetts General Hospital (MGH) / Harvard to learn from one of the leading optical coherence tomography (OCT) group in the world, just as he did during his PhD. I will never forget the countless meeting and discussion we have had at his place (no matter what time of day or night) or even inside / outside the OR.

Both Dr. Victor X.D. Yang and Dr. Michael C. Kolios have provided me with a rare opportunity to evaluate both the clinical and scientific aspects of my research. They have always given me the freedom to explore and develop my own research directions without much discouragement. I could not be more proud to have been their $\mathrm{PhD}$ student and hope that I can pass on the research values that I have picked up from them over the years.

I would also like to thank the members of my thesis committee, Dr. Xijia Gu, Dr. Dimitri Androutsos, and Dr. Mathew Kyan, who have challenged me with their insightful questions and provided invaluable suggestions. In particular, I would like to thank to Dr. Gu and his laboratory for their support over the course of my $\mathrm{PhD}$.

I am also grateful for Dr. Guillermo Tearney, Dr. Brett Bouma, Dr. Joseph Gardecki, and Dr. Milen Shishkov at MGH for our research discussions. Their insights and support have had a significant impact throughout the course of my PhD. I would especially like to thank Dr. Tearney for the numerous scholarships and research ethic board support letters.

I further like to thank Dr. Tim-Rasmus Khiel at Toronto General Hospital for training me on the art of reading histology, as well as the numerous histological processing that his laboratory has conducted for my research.

Special thanks also goes to the members of the biophotonics and bioengineering laboratory (BBL) group at Ryerson that I have worked closely with over the past five years. More specifically, I am grateful for Timothy (the clutch) Luk for his endless hours of editing my work throughout my PhD, as well as our outings with Mark (ball lenz) Harduar. Thank you to Mark Harduar, Kenneth Lee, Kyle Cheng, Antonio Mauro, Carry Sun, and Michael Leung for showing me the ropes at the beginning of my PhD. 
I am also extremely thankful for Beau Standish and Adrian Mariampillai for our endless discussions on OCT and industrial experience. Thank you to Anna Farman, Patryk Skowron, Karen Yu, Michael Lu, and Diana Varyvoda for all their help over with various animal, tissue or human experiments. I am also extremely grateful to have Joel Ramjist be apart of our research group. He had assisted in a wide variety of research and non-research related tasks at all hours of the day. In addition, he also helped me when I was injured, as well as pointed out errors whenever they arise. Another BBL member I would like to acknowledge is Helen Genis for all her hard work and dedication over the course of the summer. Without our late night discussion on issues with simulation models, we could not have been achieved in developing our solutions. As for Ronnie Wong, Jamil Jivraj, Joel Ramjist, and Nicole Dinn, thank you for not only helping me with imaging, but reminding me how much fun research can be and keeping this group close. I know you guys will achieve great things with the BBL and its unfortunate that I won't able to witness this first hand. Last but not least, the BBL wouldn't be able to run without the management of Jenny Yeow.

Finally, I would like to thank my friends and family. The amount of work and stress involved with this thesis was overwhelming at times, from imaging specimens at all hours of the night to working $100+$ hours a week revising manuscripts. I would especially like to thank Bradley Steinfeld, Gladys Maria Ruiz, Karen Gore, Danielle Mills, and Jessica Stobbe for always being there for me when times were tough. Your support has been invaluable during my research. Furthermore, thank you to Adam Kohn, Richard Rzeszutek, Ricky Leung, Thomas Behan, Aditya Menon, Paolo Auciello, and Michael Hall for the "interesting" discussions. Most of all, I thank my parents for their constant encouragement and relentless support during my studies. 


\section{Contents}

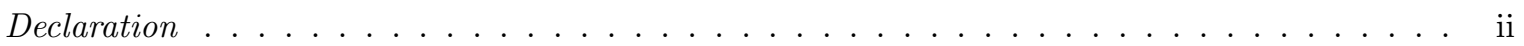

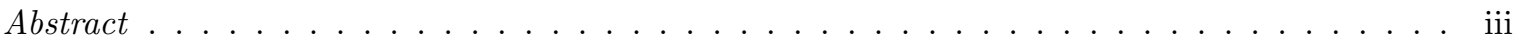

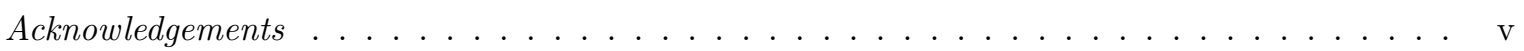

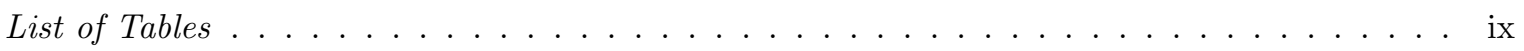

List of Figures . . . . . . . . . . . . . . . . . . . . . . . .

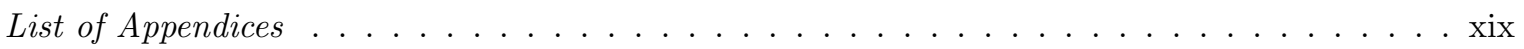

1 Introduction $\quad 1$

1.1 Intracranial aneurysm incidence, mortality and treatment options . . . . . . . . . . 1

1.2 Methods for monitoring neurovascular hemodynamic blood flow . . . . . . . . . . . . 4

1.3 Optical Coherence Tomography . . . . . . . . . . . . . . . . . . . . . . 6

1.4 Doppler Optical Coherence Tomography . . . . . . . . . . . . . . . . . . . 8

1.5 Thesis and Scientific Contributions . . . . . . . . . . . . . . . . . . . . 11

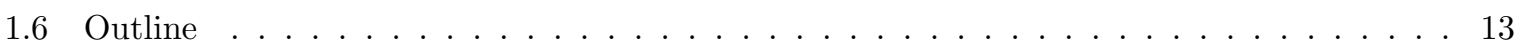

2 Intravascular split spectrum Doppler optical coherence tomography $\quad 14$

2.1 Intravascular Doppler optical coherence tomography . . . . . . . . . . . . . . . . 14

2.1.1 Intravascular Doppler optical coherence tomography system . . . . . . . . . . . 15

2.1.2 System characterization for Doppler imaging . . . . . . . . . . . 16

2.1.3 Phantom flow measurements . . . . . . . . . . . . . . . . 18

2.1.4 In vivo imaging of porcine animal model . . . . . . . . . . . . . . . . . . . . 19

2.2 Split spectrum Doppler optical coherence tomography . . . . . . . . . . . . . . 22

2.2.1 Split spectrum Doppler optical coherence tomography theory . . . . . . . . . . 23

2.2 .2 Doppler noise floor analysis . . . . . . . . . . . . . . . . . . . 29

2.2 .3 Low velocity flow phantom analysis . . . . . . . . . . . . . . . . . 31

2.2.4 In vivo split spectrum Doppler optical coherence tomography imaging . . . . . . . 33

3 Application of split spectrum Doppler optical coherence tomography in carotid stent$\begin{array}{ll}\text { ing } & \mathbf{3 7}\end{array}$

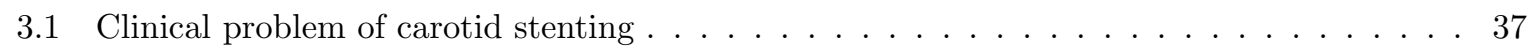


3.2 In vivo split spectrum Doppler optical coherence tomography imaging during carotid

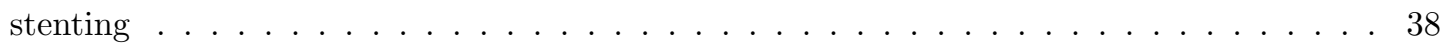

3.3 Computational fluid dynamics of stented carotid artery . . . . . . . . . . . . . . . . . . 42

4 Application of split spectrum Doppler optical coherence tomography in intracranial aneurysm flow phantoms

4.1 Intracranial aneurysm flow phantoms . . . . . . . . . . . . . . . . . . . . . . . . . . . 48

4.1.1 Simplified intracranial aneurysm flow phantoms . . . . . . . . . . . . . . . . 49

4.1.2 Patient specific intracranial aneurysm flow phantom . . . . . . . . . . . . 50

4.2 Split spectrum Doppler optical coherence tomography of an intracranial aneurysm flow

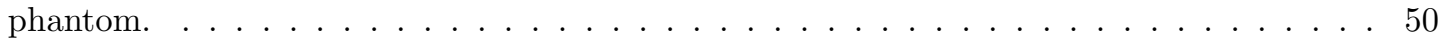

4.2.1 Imaging of a simplified intracranial aneurysm flow phantom . . . . . . . . . . . . 50

4.2.2 Imaging of patient specific intracranial aneurysm flow phantom . . . . . . . . . 51

4.3 Computational fluid dynamic models of intracranial aneurysm . . . . . . . . . . . . . . 52

4.3.1 Simplified elliptical shaped aneurysm simulations . . . . . . . . . . . . . 53

4.3.2 Patient specific aneurysm simulations . . . . . . . . . . . . . . . 55

4.3 .3 Histological findings . . . . . . . . . . . . . . . . . . . . 57

4.4 Study limitations . . . . . . . . . . . . . . . . . . . . . . . . 57

5 Ex-vivo imaging of intracranial aneurysms using optical coherence tomography 60

5.1 The natural history of intracranial aneurysms . . . . . . . . . . . . . . . . . . 61

5.2 Ex vivo structural optical coherence tomography imaging . . . . . . . . . . . . . . . . . . 61

5.2.1 Human specimens . . . . . . . . . . . . . . . . . . . . . . . 61

5.2 .2 Optical coherence tomography imaging . . . . . . . . . . . . . . . 62

5.2.3 Correlation between optical coherence tomography imaging and histology . . . . . 63

5.3 Ex vivo optical coherence tomography - optical attenuation imaging . . . . . . . . . . . . 64

5.3.1 Medulloblastoma animal model . . . . . . . . . . . . . . . 66

5.3.2 Optical coherence tomography - optical attenuation imaging . . . . . . . . . . . 66

5.3.3 Medulloblastoma and optical coherence tomography - optical attenuation . . . . 68

5.3.4 Intracranial aneurysm and optical coherence tomography - optical attenuation . . 68

5.3.5 Limitation of optical coherence tomography - attenuation imaging . . . . . . . . . 72

6 Summary, Conclusion, and Future Work 74

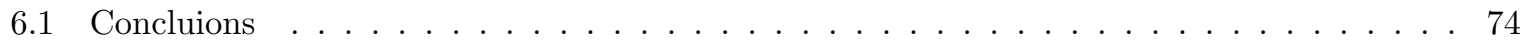

6.2 Future work ............................ . . . . . . . . . . . . . . .

\begin{tabular}{ll} 
References & 103 \\
\hline
\end{tabular} 


\section{List of Tables}

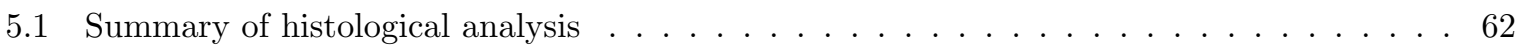

5.2 Summary of optical attenuation coefficient by tissue type [1]. (Reprinted with permission

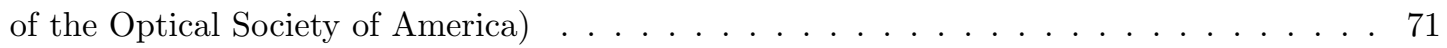




\section{List of Figures}

1.1 Anatomical representation of the circle of Willis. Courtesy of Jay Edry. . . . . . . . . . 2

1.2 A photograph of a clipped intracranial aneurysm. . . . . . . . . . . . . . 3

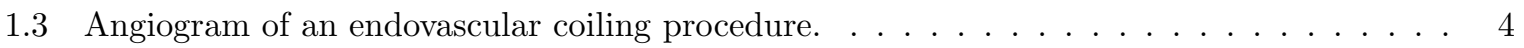

1.4 Simplified schematic of a TD-OCT system. Coupled light from a low coherence source is directed to a beam-splitter (BS). Half the light is split to a translating reference mirror and the remaining half is directed to a sample arm, which interrogates the tissue. The retroflected light is measured at the detector. . . . . . . . . . . . . . . 7

1.5 (a) The hematoxylin and eosin (H\&E) stained histologic of a human intracranial aneurysm. Layers (arrows) and calcium deposits $\left({ }^{*} \mathrm{Ca}\right)$ can be observed. (b) The corresponding structural OCT image. Scale bar: $1 \mathrm{~mm} . \ldots \ldots \ldots \ldots \ldots$

1.6 (a) A representation of the detected OCT A-scan from a single scatterer moving through the optical beam. (b) Sampled dataset of the OCT A-scan in slow time axis. . . . . . . . .

1.7 (a) Structural OCT image of a HHT patients fingertip with abnormal vasculature. (b) The corresponding DOCT image shows blood flowing through superficial micro vessels (arrows). Scale bar: $1 \mathrm{~mm} \mathrm{\ldots} \mathrm{.} \mathrm{.} \mathrm{.} \mathrm{.} \mathrm{.} \mathrm{.} \mathrm{.} \mathrm{.} \mathrm{.} \mathrm{.} \mathrm{.} \mathrm{.} \mathrm{.} \mathrm{.} \mathrm{.} \mathrm{.} \mathrm{.} \mathrm{.} \mathrm{.} \mathrm{.} 10$

1.8 Regenerated dataset of normalized standard deviation versus flow velocity (without Doppler angle correction). Each data point is the mean of 25,000 pixels $[2] \ldots \ldots \ldots \ldots$. . . . 11

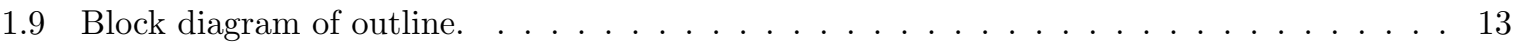

2.1 Schematic of the the custom data acquisition system. The interference OCT signal, Aline trigger (Trig) and k-clock (Clk) are coupled out to a PC based data acquisition card. The interference OCT signal is recorded on a high speed storage drive, while the graphical processing unit (GPU) performs inverse fast Fourier transforms to provide real time structural OCT images to a display. . . . . . . . . . . . . . . . 16

2.2 (a) Schematic of the C7 Dragonfly. The distal end of the catheter consists of a microprism fused to a GRIN fiber, coreless fiber, and single mode fiber (SMF). All optics were encased in an inner catheter sheath. (b) A photograph of the C7 Dragonfly catheter. . . . . . . 17

2.3 (a) OCT image of a stationary phantom. (b) Doppler OCT image without bulk motion compensation. (c) Doppler OCT image with bulk motion compensation. Scale bar 1mm. . 17 
2.4 Imaging of a slow flow phantom. (a) Structural image of $1.5 \%$ blood in saline mixture within the tube, where arrow A indicates the internal reflection of the imaging optics, and arrow B indicates the outer surface of the imaging catheter sheath. (b) Phase shift obtained from the internal reflection indicated by arrow A and catheter sheath indicated by arrow B in (a). The phase shift of the catheter sheath is the median of data along the catheter thickness. (c) Doppler shift image of the slow flow phantom. The dashed ring indicates the sheath of the imaging catheter, with phase shift induced by NURD in a radially constant manner throughout the image. (d) Doppler image after suppression of the motion artifact, which displays the phase shift induced by the slow flow inside the tube phantom. Scale bars $=1 \mathrm{~mm}$ [3]. (Reprinted with permission of the Optical Society

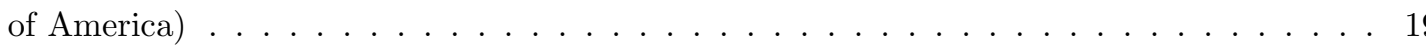

2.5 Imaging of a slow flow phantom at various blood and saline concentration. (a) DOCT image of $10 \%$ blood in saline mixture within the tube. (b) DOCT image of a blood in saline mixture of $5 \%$. (c) DOCT images were then taken after the blood in saline mixture was reduced to $2.5 \%$. (d) Finally, DOCT image acquired with a blood in saline mixture

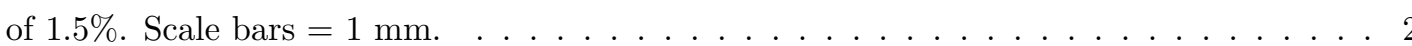

2.6 In vivo endovascular flow measurement. (a) 3D OCT image of the catheter and the vessel wall, which shows the angle between the catheter and the wall is $\sim 10^{\circ}$. (b) Simulation results of blood flow. (c) Cross-sectional OCT image of a porcine carotid artery with shadow casted by guide wire. (d) The same cross-section as (c) imaged by 500 A-lines/frame with Doppler shift overlaid, showing mainly noise. (e) and (f) (Media 1). (e) Phase shift image, 2500 A-lines/frame, without NURD induced phase artifact removal. It shows distorted phase contour lines. (f) Phase shift image after NURD induced phase artifact removal by tracking the phase shift in the catheter sheath. The corrected phase contour lines are as expected. The NURD induced artifact is time variant, as shown by Media 1. (g) A typical cross-sectional frame, 2500 A-lines/frame with Doppler shift overlaid, showing aliased phase changes. (h) The unwrapped phase map of (g), where * indicates the highest velocity region. The arrows indicate incorrect phase unwrapping due to noise and high shear rate near the vessel wall. Scale bars $=1 \mathrm{~mm}$ [3]. (Reprinted with permission of the Optical Society of America) . . . . . . . . . . . . . . . . 21

2.7 A simplified diagram that depicts two square wave (blue and red box) pulses emitting from an ultrasound transducer (top diagram). The result of the autocorrelation of these square waves results in a triangle waveform, which was used to derived the standard deviation of the Doppler shift using Eq. 2.1. It can be seen that a longer pulse length $\left(T_{p}\right)$ can result in a decrease in standard deviation given a transducer center frequency $\left(f_{o}\right)$ and the speed of sound (c). However, an increase in pulse repetition frequency $\left(f_{p r f}=\frac{1}{T_{p r f}}\right)$ and velocity of the moving scatterer $\left(v_{z}\right)$ would increase the standard deviation of the

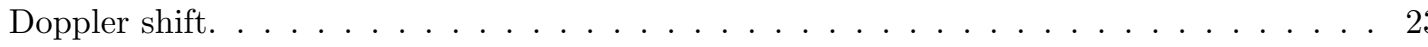


2.8 Normalized standard deviation of phase shift $\left(\sigma_{\Delta \Phi}\right)$ versus flow velocity. The result of Equation 2.22 with the coherence length $\left(l_{c}\right)$ of the system from [2] was plotted. Coherence length was increased by a factor of 2 and 4 (spectral bandwidth decreases to $50 \%$ and $25 \%$ ) via fractional bandwidth (FBW) and the respective phase noise $\left(\sigma_{\Delta \Phi}\right)$ of were plotted as well [4]. (Reprinted with permission of the Optical Society of America) . . . . . . . . . . 27

2.9 Data acquisition and processing scheme. The OCT rotary catheter was inserted into a vessel and images were acquired during blood flow. Multiple narrow window bands with different center frequencies were multiplied with the interferogram. These windowed spectra were stored into the corresponding windowed band frame and the mean phase shift was calculated by evaluating the phase difference between axial scans within the frame (Equation 2.23) [4]. (Reprinted with permission of the Optical Society of America) 28

2.10 A series of 20 structural OCT images were taken in B-mode of a stationary tissue phantom. (a) and (b): mean measurements of background phase noise (standard deviation of $\Delta \Phi$ ) using DOCT and ssDOCT at different fractional bandwidth (FBW) for high SNR(approx. $6 \mathrm{~dB}$ ) region and low SNR (approx. 2dB) region at an ensemble length $\mathrm{N}=10$. In all ssDOCT calculations, $M=1$. The catheter beam was then set at a static position and 20 structural OCT images were taken in M-mode. (c) and (d): mean measurements of background phase noise using DOCT and ssDOCT at various ensemble lengths (N) for high SNR region and low SNR region [4]. (Reprinted with permission of the Optical Society

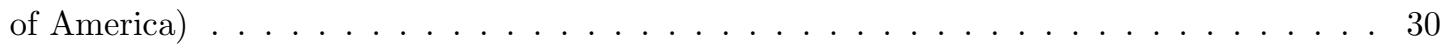

2.11 Structural image of a slow flow phantom with $1.5 \%$ diluted blood mixture realized using the full length apodization window $(B=1, M=2, N=10)$. (b) The corresponding structural SSP-OCT image $(B=4, M=1, N=10)$. (c) and (d) The Doppler shift of the slow flow phantom evaluated by DOCT and ssDOCT, respectively. Doppler artefacts were present in both images. (e) and (f) The corrected Doppler image computed from DOCT and ssDOCT techniques, respectively. The relative phase from (g) DOCT and (h) ssDOCT was then visualized by subtracting the averaged velocity profile. More phase noise is present in DOCT compared to ssDOCT. Scale bar $=1 \mathrm{~mm}$ [4]. (Reprinted with permission of the Optical Society of America) . . . . . . . . . . . . . . . . 32

2.12 (a) An ANSYS simulation fitted phase shift model of Figure 2.11 was generated. Scale $=$ $1 \mathrm{~mm}$ (b) The axial profile was taken (dashed line in (a)) from the center of the catheter (arrow) and the mean \& standard deviation were compared. The expected velocity profile was then plotted against the measured phase shift of (c) DOCT ( $B=1, M=2, F B W=100 \%)$ and (d) $\operatorname{ssDOCT}(\mathrm{B}=4, \mathrm{M}=1, \mathrm{FBW}=44 \%)$. The expected phase shift (red) and mean $\&$ standard deviation (blue) were plotted [4]. (Reprinted with permission of the Optical Society of America) . . . . . . . . . . . . . . . . . . . . . . 33 
2.13 The structural OCT image overlapped with Doppler image of a high flow region in a porcine carotid artery. (a) Doppler images produced by DOCT ( $\mathrm{B}=1, \mathrm{M}=4, \mathrm{FBW}=100 \%)$. (b) The corresponding $\operatorname{ssDOCT}(\mathrm{B}=2, \mathrm{M}=1, \mathrm{FBW}=60 \%)$ with approximately similar phase noise performance as shown in Figure 2.10(a). The arrows indicate less degradation in the visualization of the aliasing rings comparing (b) to (a). The unwrapped value in both DOCT and ssDOCT showed a peak velocity of $18.7 \pi$ (asterisk). Scale bar $=1$ mm [4]. (Reprinted with permission of the Optical Society of America) . . . . . . . . . . 34

2.14 (a) Structural image of a deployed stent in the carotid artery using the full length apodization window $(\mathrm{B}=1, \mathrm{M}=4, \mathrm{~N}=20)$. (b) The corresponding structural SSP-OCT image $(\mathrm{B}=2$, $\mathrm{M}=1, \mathrm{~N}=20, \mathrm{FBW}=60 \%$ ). (c) and (d)The corrected Doppler image computed from DOCT and ssDOCT techniques, respectively. (e) DOCT and (f) ssDOCT were thresholded at the same phase value. Note the two detected flow profiles (upper * \& lower *). The upper flow profile $\left(^{*}\right)$ may be caused from a stent strut upstream from the location of the OCT struts. Scale bar $=1 \mathrm{~mm}[4]$. (Reprinted with permission of the Optical Society

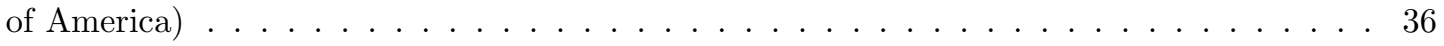

3.1 (a) Fluoroscopic image of the stent placement. (b) Contrast was injected into the artery in order to visualize the gross flow distribution of the vessel after stent intervention. Scale bar 10mm. (c) Magnified view of the region marked with the dashed box in (a), showing details of catheter and guidewire positions. (d) Magnified view with contrast injection, showing blood in-flow, mild spasm distal to the stent edge in an out-flow branch. Notice in this particular setting, the OCT imaging catheter and its guidewire are placed through the stent in one of the out-flow branches. Scale bar: $3 \mathrm{~mm}[5]$. (Reprinted with permission

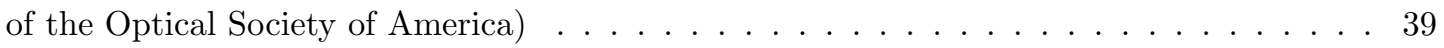

3.2 (a) In vivo structural OCT image of the carotid artery before stent deployment. (b) The corresponding ssDOCT image. Scale bar: $1 \mathrm{~mm}$. (c) Magnification at the neck of the carotid artery (dashed box). Scale bar: $0.5 \mathrm{~mm}$. [5]. (Reprinted with permission of the Optical Society of America) . . . . . . . . . . . . . . . . . . . . 40

3.3 (a) In vivo structural OCT image of the ICA after stent implantation. (b) The corresponding ssDOCT image. A magnification of the ssDOCT image revealed phase contours that envelope the stent struts. Scale bar: $1 \mathrm{~mm}[5]$. (Reprinted with permission of the Optical Society of America) . . . . . . . . . . . . . . . . . . . . 40

3.4 (a) In vivo structural OCT image of a protruded stent strut at the neck of the side branch.

(b) The corresponding ssDOCT image, where the dashed box indicates the protruded stent strut. (c) This magnified region of interest depicts a slow velocity profile (triangle) (d) After $1.25 \mathrm{~s}$, it was noted that there was a formation of an eddy current (arrow). (e) At 1.55 s from the initial frame, low to stagnant velocity measurements were observed $(*)$. Scale bar $1 \mathrm{~mm}$ [5]. (Reprinted with permission of the Optical Society of America) . . . . 41 
3.5 (a) In vivo structural OCT image after stent deployment in the ICA. (b) The corresponding ssDOCT image. (c) In vivo structural OCT image of backflow from the side branch to the main branch. (d) The corresponding ssDOCT image of the backflow. Scale bar: 1 mm [5]. (Reprinted with permission of the Optical Society of America) . . . . . . . . . . 42

3.6 (a) Structural OCT image in the transverse plane, demonstrating the parent vessel (*pv), side branch $\left({ }^{*} \mathrm{~b}\right)$, and the stent struts with shadows. (b) Reconstructed longitudinal plane of the blood vessel from OCT pull-back imaging was employed as a reference for the 3D geometry of the CFD model. (c) The velocity distribution before stent implantation with the OCT catheter from the CFD simulation of the carotid artery in the transverse plane with flow velocity contours. (d) Streamlines overlaid on the CFD simulated velocity distribution with the OCT catheter in the longitudinal plane. (e) The CFD simulation of the velocity distribution before stent placement without the OCT catheter. (f) The corresponding streamlines that was overlaid on the CFD simulated velocity distribution without the OCT catheter in the longitudinal plane. Scale bar: $1 \mathrm{~mm}$ [5]. (Reprinted with permission of the Optical Society of America) . . . . . . . . . . . . . 43

3.7 (a) The velocity distribution from the CFD simulation with the OCT catheter present after stent implantation covering the bifurcation in the longitudinal plane. Streamlines were overlaid onto the velocity distribution. An eddy was observed close to the stent strut near the entrance of the bifurcation (dashed region). (b) The corresponding CFD simulation without the OCT catheter within the lumen wall. Similarly, an eddy current was also observed around the stent strut near the entrance of the bifurcation (dashed region) (c) Contralateral to the position of the OCT catheter, a recirculation zone near a stent strut was observed. (d) The CFD simulation was re-sliced to view the distribution in the transverse plane with the OCT catheter. (e) The corresponding CFD simulation without the OCT catheter image. Scale bar: $1 \mathrm{~mm}[5]$. (Reprinted with permission of the Optical Society of America) . . . . . . . . . . . . . . . . . . . 46

3.8 (a) The normalized inlet velocity profile used during CFD modeling. Peak velocity (black arrow) was simulated in Figure 3.7, while ssDOCT were observed during late diastole (Green arrows) (b) The cross section of the CFD with an inlet velocity of $20 \mathrm{~cm} / \mathrm{s} \mathrm{(c)}$ Structural OCT image when the OCT catheter was place distally near the carina (d) The corresponding ssDOCT image. Scale bar: $1 \mathrm{~mm} . \ldots \ldots \ldots \ldots$. . . . . . . . . 47

4.1 (a) Photo of the stented simplified intracranial aneurysm flow phantom. (b) Photo of the patient specific intracranial aneurysm flow phantom. Scale bar: $5 \mathrm{~mm}$. . . . . . . . . 49

4.2 A CT image of a 63-year-old female with a PCA aneurysm. Scale bar: $5 \mathrm{~cm} . \quad \ldots \ldots$. . . 50

4.3 (a) OCT structural image of a simplified aneurysm model before stent implantation during the systolic cycle. (b) The corresponding ssDOCT image. Low velocities estimates were resolved at the neck of the aneurysm. (c) OCT structural image of a simplified aneurysm during the diastolic cycle. (d) The corresponding ssDOCT image. Majority of the flow profiles were mid to low velocities. Scale bar: $1 \mathrm{~mm} . \ldots \ldots \ldots \ldots$. . . . . . . 51 
4.4 (a) OCT structural image of a simplified aneurysm model after stent implantation during the systolic cycle. (b) The corresponding ssDOCT during systolic cycle. Low velocities were observed at the neck of the aneurysm. (c) The structural OCT image during diastolic cycle. (d) The corresponding ssDOCT during diastolic cycle. The laminar-like profile was observed in the neck of the aneurysm, whereas the parent vessel exhibited asymmetric flow profile. Scale bar: $1 \mathrm{~mm} \ldots \ldots \ldots \ldots \ldots$. . . . . . . . . . . . 52

4.5 (a) Structural OCT image of a patient-specific flow phantom during the diastolic flow. (b) The corresponding ssDOCT image. Two distinct flow regions were observed. (c) Structural OCT image of a patient-specific flow phantom during the systolic flow. (d) The corresponding ssDOCT image demonstrated higher flow velocity (increased phase contours). Separation of flow contours was also observed (dashed line). Scale bar: $1 \mathrm{~mm}$.

4.6 (a) Reconstructed longitudinal plane of the CFD elliptical aneurysm model during the systolic cycle. (b) Reconstructed longitudinal plane of the CFD elliptical aneurysm model during the diastolic cycle. (c) The corresponding longitudinal plane of the CFD elliptical aneurysm model during systolic cycle with the OCT catheter (d)The corresponding longitudinal plane of the CFD elliptical aneurysm model during the diastolic cycle with the OCT catheter (e) The cross sectional plane of the elliptical aneurysm model during the systolic cycle. (f) The cross sectional plane of the elliptical aneurysm model during diastolic cycle. (g) The corresponding cross sectional plane of the elliptical aneurysm model during the systolic cycle with the OCT catheter. (h)The corresponding cross sectional plane of the elliptical aneurysm model during diastolic cycle with the OCT catheter. Scale

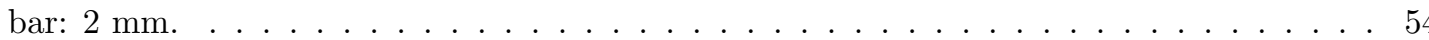

4.7 (a) Reconstructed longitudinal plane of the CFD elliptical aneurysm model after virtual stent deployment during the systolic flow. An inflow stream had formed due to the stent strut which diverted flow into the aneurysm sac (arrow). (b) The CFD elliptical aneurysm model after virtual stent deployment during the diastolic flow. (c) The corresponding systolic CFD model with the OCT catheter. (d) The resulting CFD model during diastolic flow with the OCT catheter. (e) The corresponding CFD elliptical aneurysm model after virtual stent deployment during the systolic flow in the transverse plane. (f) The corresponding the CFD elliptical aneurysm model after virtual stent deployment during the diastolic flow in the transverse plane. (g) The CFD model after the introduction of the OCT catheter during systolic flow. (h) The corresponding CFD model with the OCT catheter during diastolic cycle. Scale bar: $2 \mathrm{~mm} . \ldots \ldots \ldots \ldots$

4.8 (a) The ssDOCT image within an elliptical aneurysm model acquired just after peak velocity. A deviation in phase contours $(*)$ was observed with respect to the contours within the neck of the aneurysm. Scale bar: $1 \mathrm{~mm}$. (b) The corresponding CFD elliptical aneurysm model at the location of a vortex. The velocity dynamic range was decreased to simulate phase aliasing, which results in a separation of contours $\left(^{*}\right)$. Scale bar: $2 \mathrm{~mm} . \quad 57$ 
4.9 (a) Pre-operative model of a patient specific aneurysm CFD during the systolic cycle. (b) Pre-operative model of a patient specific aneurysm CFD during the diastolic cycle. The presence of a vortex was observed (arrow) during the systolic and the diastolic cycle (c) Cross sectional view of the patient specific aneurysm CFD during the systolic cycle. (d) Cross sectional view of the patient specific aneurysm CFD during diastolic cycle. Scale bar: $2 \mathrm{~mm} \ldots \ldots \ldots \ldots \ldots \ldots \ldots \ldots$

4.10 (a) Histologic sections of aneurysm stained with H\&E. Boxed regions show site of ruptured wall with extravasated blood. (b) The magnification of boxed region. (c) Corresponding histologic section stained with Movat pentachrome. Movat is a stain that highlights connective tissue. (d) Movat stain with 10x magnification of boxed area. . . . . . . . . . . 59

5.1 (a) $5 \mu \mathrm{m}$ section of a ruptured cerebral aneurysm sample from the ophthalmic artery $(\mathrm{H} \& \mathrm{E})$. Dense Fibrotic $\left({ }^{*} \mathrm{~F}\right)$ and hyaline $\left({ }^{*} \mathrm{Hy}\right)$ regions were observed throughout the specimen. (b) 10x magnification of the thin hyalinized wall and fibrotic $\left({ }^{*} \mathrm{~F}\right)$ regions. (c) There is a strong delineation between the thin wall and the collagenous layer in the OCT image. (d) Corresponding OCT magnification. Delineation between the Dense Fibrotic $\left({ }^{*} \mathrm{~F}\right)$ and thin hyalinized wall $\left({ }^{*} \mathrm{Hy}\right)$ was observed. Scale bar is $1 \mathrm{~mm} \ldots \ldots$. . . . . . . . . 63

5.2 (a) $5 \mu \mathrm{m}$ section of an unruptured cerebral aneurysm sample from the pericallosal artery, stained with H\&E. Various stages of fibrosis, such as regions of fibrotic tissue $\left({ }^{*} \mathrm{~F}\right)$ was observed throughout the sample. The presence of calcium $\left({ }^{*} \mathrm{Ca}\right)$ and dense hemosiderin $\left({ }^{*} \mathrm{H}\right)$ deposits suggests a healing process of the vessel. Hemosiderin is commonly found in vessels that have experienced prior hemorrhages. The deep purple area observed is the lumen of an organized thrombus (*OT; clear material on luminal surface is $2 \%$ agarose gel, related to OCT analysis). (b) OCT rendition of the aneurysm specimen. The hemosiderin $\left({ }^{*} \mathrm{H}\right)$ is clearly visible in the optical image. Additionally, the boundary between collagenous tissue and hemosiderin ${ }^{*} \mathrm{H}$ ) can be resolved. However, the calcium deposits could not be visualized due to attenuation of light through the degenerated tissue. (c) 10x magnification of the region with hemosiderin $\left({ }^{*} \mathrm{H}\right)$ and fibrosis $\left({ }^{*} \mathrm{~F}\right)$. (d) Corresponding OCT magnification. Alignment of hemosiderin $\left({ }^{*} \mathrm{H}\right)$ along the adventitial side was resolved. Furthermore, the delineation between the fibrotic $\left({ }^{*} \mathrm{~F}\right)$ and hyalinized regions was observed. (e) 3-dimensional OCT visualization of the intracranial aneurysm. Digital resection can be achieved (green dotted line) in the volumetric dataset. (f) This resulted in the detection of clusters of hemosiderin. Scale bar is $1 \mathrm{~mm} \ldots \ldots \ldots$. . . . . . . . 65 
5.3 (a) Gross histology of harvested Math1-GFP;Ptch ${ }^{+/-}$mouse model. (b) The corresponding fluorescence image. (c) The combined gross and fluorescence image. Normal cerebellum (cyan line) and an interface of normal/medulloblastoma (red line) scans were taken. (d) $\mathrm{H} \& \mathrm{E}$ section of the cerebellum. Grey matter consisting of molecular layer $\left({ }^{*} \mathrm{M}\right)$ and granular layer $\left({ }^{*} \mathrm{G}\right)$ was observed. Underneath the granular layer, regions of white matter are present $\left({ }^{*} \mathrm{~W}\right)$. (e) The associated structural OCT with minimal resolved features. (f) The corresponding OCT-OA image consisted of a layered structure. The shape and location of these layers are similar to the molecular layer $\left({ }^{*} \mathrm{M}\right)$ and granular layer $\left({ }^{*} \mathrm{G}\right)$. Scale bar $500 \mu \mathrm{m}$ [1]. (Reprinted with permission of the Optical Society of America) . . 69

5.4 (a) H\&E section of the cerebellum in a Math1-GFP;Ptch ${ }^{+/-}$model. Grey matter consisting of molecular layer $\left({ }^{*} \mathrm{M}\right)$ and granular layer $\left({ }^{*} \mathrm{G}\right)$ was observed. Underneath the granular layer, regions of white matter $\left({ }^{*} \mathrm{~W}\right)$ are present. Regions of medulloblastoma resided on the right side $\left({ }^{*} \mathrm{~T}\right)$. Within the red dashed box, medulloblastoma regions that were adjacent to normal cerebellum demonstrated very dense cellular proliferation (red arrow). On the right side of the medulloblastoma region, superficial cells appear to be sparsely spaced (green arrow); however a lining of dense cells was located below. (b) The corresponding structural OCT with minimal resolved features. (c) The OCT-OA image consisted of a layered structure and erratic high attenuation region. In normal tissue the layers consisted of the molecular layer $\left({ }^{*} \mathrm{M}\right)$ and granular layer $\left({ }^{*} \mathrm{G}\right)$. The erratic high attenuation region was where medulloblastoma $\left({ }^{*} \mathrm{~T}\right)$. Scale bar $500 \mu \mathrm{m}[1]$. (Reprinted with permission of the Optical Society of America) ～. . . . . . . . . . . . 70

5.5 (a) H\&E stained section of an unruptured intracranial aneurysm. A large density of hemosiderin $\left({ }^{*} \mathrm{H}\right)$ was observed. Scale bar: $1 \mathrm{~mm}$ (b) Magnification of the aneurysm wall. Irregular fibrous thickening $\left({ }^{*} \mathrm{IF}\right)$ along the vessel wall was observed. Scale bar: $200 \mu \mathrm{m}$. (c) Structural OCT image of the unruptured intracranial aneurysm. (d) The corresponding uncalibrated OCT-OA image. Scale bar: $1 \mathrm{~mm} . \quad \ldots \ldots \ldots \ldots$. . . . . 71

5.6 (a) H\&E stained section of a ruptured intracranial aneurysm. (b) Structural OCT image

(c) The corresponding uncalibrated OCT-OA image. Scale bar: $1 \mathrm{~mm} . \ldots \ldots$. . . . . . 72

6.1 A computer tomography image of a 74-year-old male after stenting. It was observed that the stent was not completely deployed on the carotid artery wall (arrow). Scale bar: $1 \mathrm{~cm} .77$

6.2 (a) Structural OCT image of a stented carotid artery. Coagulated blood was artificially injected into the vessel and attached to the stent struts (arrows). (b) The corresponding ssDOCT image. Scale bar: $1 \mathrm{~mm} . \ldots \ldots \ldots \ldots \ldots$. . . . . . . . . . . . . . . . . . 
6.3 OCT imaging from an in-vivo human airway. (a) Structural OCT image with DOCT overlay ( $\mathrm{B}=1, \mathrm{M}=8, \mathrm{~N}=32)$. (b) Split spectrum processing $(\mathrm{B}=4, \mathrm{M}=2, \mathrm{~N}=32, \mathrm{FBW}=60 \%)$ of the same imaging frame. Both images have identical Doppler display thresholds. The green circle indicates a true blood vessel confirmed by continuity between imaging frames. The red circle shows a Doppler artefact that was suppressed in the split spectrum processed image. The color-coded CDOCT scale bar spans $+/-8.5 \mathrm{~mm} / \mathrm{s}$. Scale bar: 0.5 mm [4]. (Reprinted with permission of the Optical Society of America) . . . . . . . . . 78

6.4 (a) A longitudinal view of in-vivo human airway. The Doppler image was overlaid onto the structural OCT image. A single blood vessel (arrow) could be clear resolved within the lung. Scale bar: $100 \mu \mathrm{m} . \ldots \ldots \ldots$. . . . . . . . . . . . . . . 79

3.1 Work flow chart of the tissue preparation and imaging used in this study. [6] $\ldots \ldots 6$

3.2 (a) Fiducial markers were cut into the agarose. (b) This represented starting position of

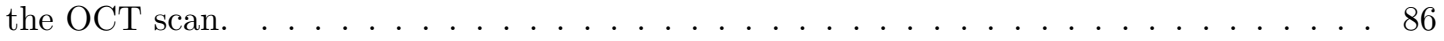

4.1 Flow diagram of a mean shift image segmentation based optical coherence tomography optical attenuation imaging . . . . . . . . . . . . . . . 87 


\section{List of Appendices}

1 Derivation of the Kasai velocity estimaton $\quad 80$

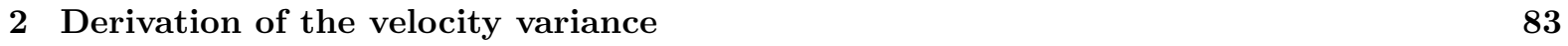

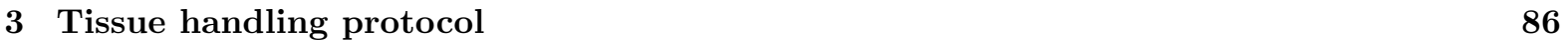

4 Optical coherence tomography - optical attenuation flow diagram $\quad 87$

5 Permission $\quad \mathbf{8 8}$

5.1 The Optical Society of America (Optics Express and Biomedical Optics Express) . . . . 88

6 List of Contributions $\quad 89$ 


\section{Chapter 1}

\section{Introduction}

This introductory chapter provides the background and motivations for this thesis, including the prevalence of intracranial aneurysms, current treatment options, optical coherence tomography, and Doppler optical coherence tomography as an imaging modality capable of measuring and monitoring hemodynamic changes.

\subsection{Intracranial aneurysm incidence, mortality and treatment options}

One of the most catastrophic events that could occur in the neurovascular system is the rupture of an intracranial aneurysm. Following rupture, spontaneous subarachnoid hemorrhage (SAH) occurs, possibly leading to the onset of severe headaches, unconsciousness and neurological deficits [7]. In North America and Western Europe, SAH has an incidence rate of approximately 10-11 cases per 100,000 people per year. In the general population the incidence rate can increase up to 35 cases per 100,000 people per year, where the Finnish and Japanese populations are known to have one of the highest incidences. Between the years of 1987 and 1992, the mortality rate decreased by 10\% [7]. At the present time, it is believed that improvements in mortality may be due to the advancements in surgical techniques, advancement in endovascular devices and aggressive management of complications from SAH [8].

An intracranial aneurysm is a bulging, "berry-like" deformity of the vessel wall that typically originates at a bifurcation in the circle of Willis, a network of arteries that supply blood to the brain (Figure 1.1). Currently, the mechanism of aneurysm formation is not well understood. It is believed that the direct hemodynamic stress from the blood flow is the key contributing factor to the thinning or degeneration of the focal point leading to early stage formation of aneurysms. This demonstrated good correlation with the reported increase in the number of detected aneurysms at the posterior communicating artery (30 - 35\%), anterior communicating artery (30 - 35\%), bifurcation of internal carotid, and bifurcation of middle cerebral artery $(20 \%)$. The degeneration of the focal point leading to the formation of an intracranial aneurysm has also been linked to heritable connective tissue diseases, such 


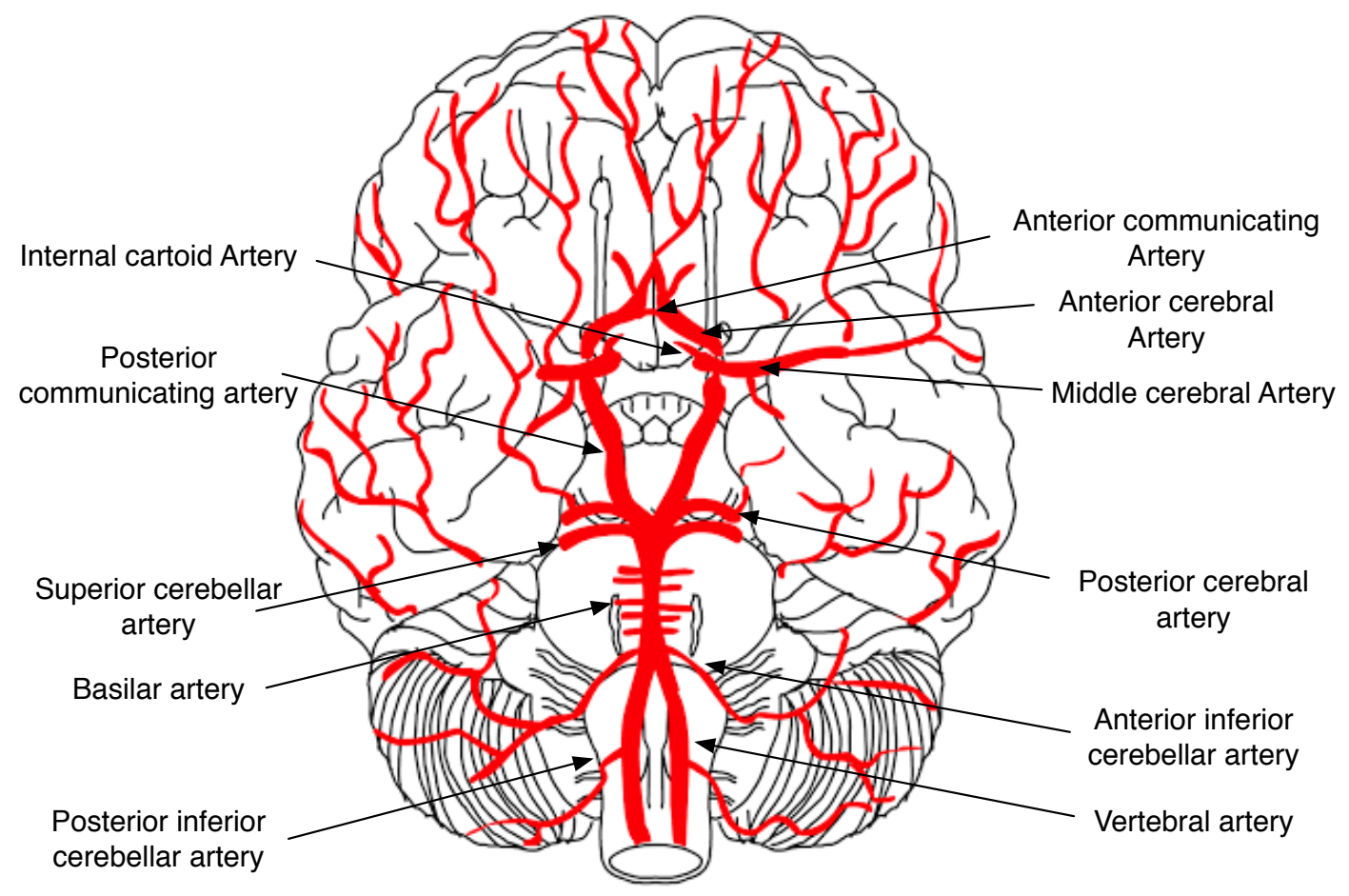

Figure 1.1: Anatomical representation of the circle of Willis. Courtesy of Jay Edry.

as polycystic kidney/liver disease, Ehlers-Danlos syndrome, Marfan syndrome, hereditary hemorrhagic telangiectasia (HHT), neurofibromatosis type 1, pseudoxanthoma elasticum, and tuberous sclerosis [9].

In general, no specific symptoms are associated with unruptured aneurysms and they are often accidentally detected. Patients diagnosed with an unruptured aneurysm are subjected to angiography to assess the size, shape, and location to determine the risk of rupture and the appropriate course of treatment. More specifically, it is well established that the anterior communicating artery and pericallosal artery are more likely to rupture than the middle cerebral artery [10]. In terms of the size, aneurysms measuring between 7 to $12 \mathrm{~mm}$ (posterior communicating artery) have a 14.5\% risk of rupture (5 - year risk), whereas aneurysm $<7 \mathrm{~mm}$ have a $3.4 \%$ risk (with previous SAH) [11]. Treatment of aneurysms predominately focuses on the prevention of SAH with physicians considering the aforementioned angiographic factors, as well as patient factors and subjective surgical and interventional experiences during the decision making process. Common standard of care treatments of intracranial aneurysms include microsurgical clipping and stenting/coiling. The following paragraphs summarize these treatments, their benefits, and associated risk.

Microsurgical clipping is an invasive approach to isolate and treat intracranial aneurysms. This treatment involves the temporary removal of bone from the skull to gain access to the brain. Once 


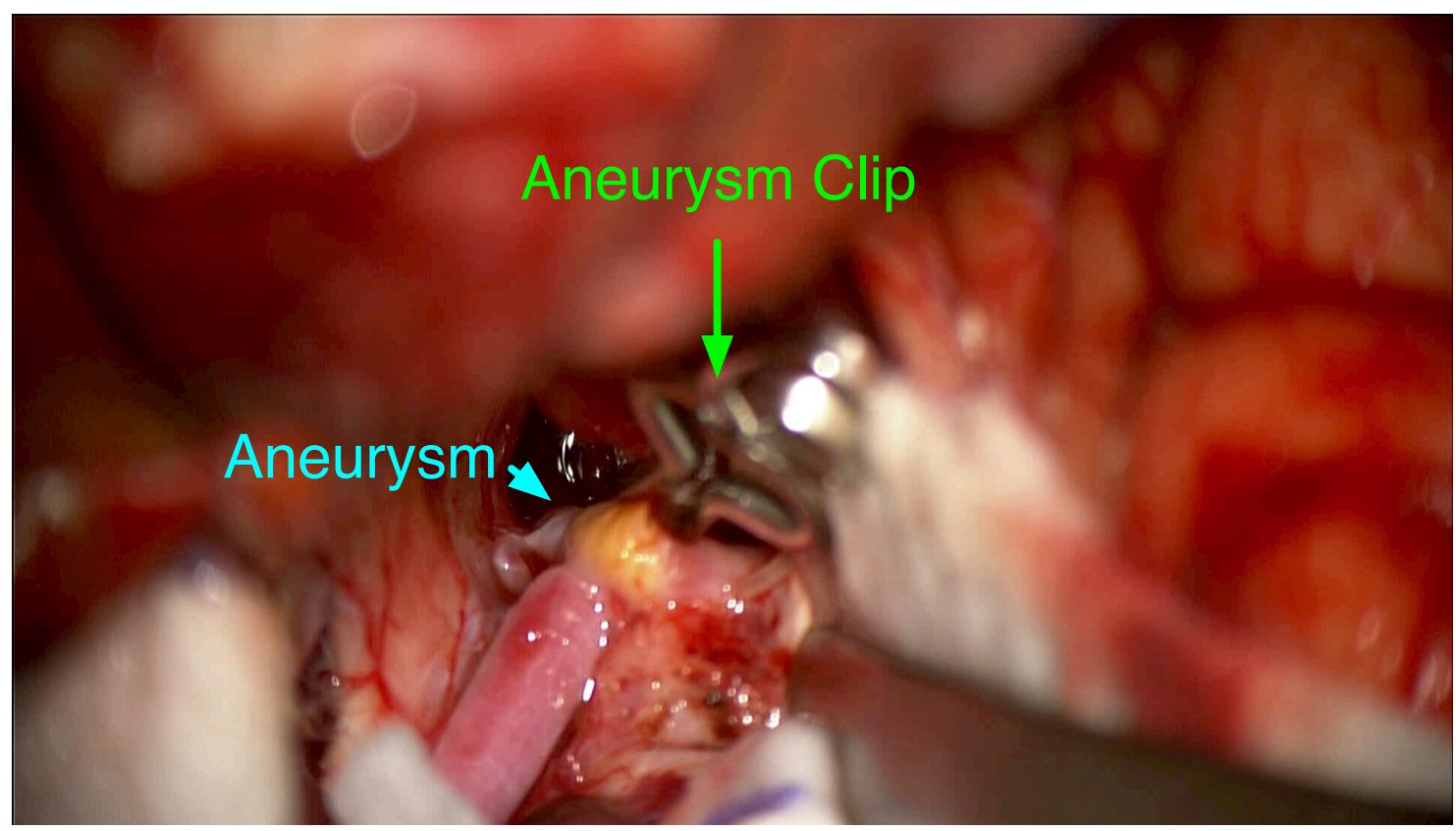

Figure 1.2: A photograph of a clipped intracranial aneurysm.

the aneurysm is located, a titanium clip is placed across the neck of the aneurysm in order to restrict the flow of blood from entering. Finally, the resection of the aneurysm is sometimes performed, while the clip is permanently situated in the brain (Figure 1.2). Within in the first 5 years of microsurgical clip placemement, the incidence of a SAH has been reported to be between $1 \%$ and $2 \%$ [12-14]. However, reformation of aneurysms has been reported to be $10 \%$ after nine years post-operative clipping intervention, which could lead to another SAH [15].

Endovascular coiling is an emerging treatment option for aneurysms that was first introduced in 1990 and is considered to be minimally invasive. During the treatment, a small incision is made into the femoral artery. Catheters are then guided through the blood vessels to the neck of the aneurysm by fluoroscopy. Contrast agent is injected into the blood vessels to assist in visualization of the aneurysm and its peripheral vessels. The tip of a microcatheter is steered to the neck of the aneurysm and platinum coils are deposited into the sac (Figure 1.3). Coils are compacted into the aneurysm until flow into the aneurysm is obstructed. If the width of the neck of the aneurysm is wide ( $>4 \mathrm{~mm})$ or irregular in shape, stents are deployed to ensure coil placement. Several complications associated with aneurysm coiling can occur such as the formation of blood cots (thrombogenesis), vasospasm (narrowing of blood vessel), or the reformation of the aneurysm. The reoccurrence of aneurysms after this treatment has reported to be as high as $34 \%$ [16]. As a result, it has been recommended that patients should follow-up with angiographic monitoring.

Flow diverting stents, also referred to as pipeline embolization devices (PED) were first approved for clinical use in 2011 by the food and drug administration (FDA). These PEDs have been used to treat complex aneurysm cases, often associated with large sizes and wide necks. The goal of the PED 


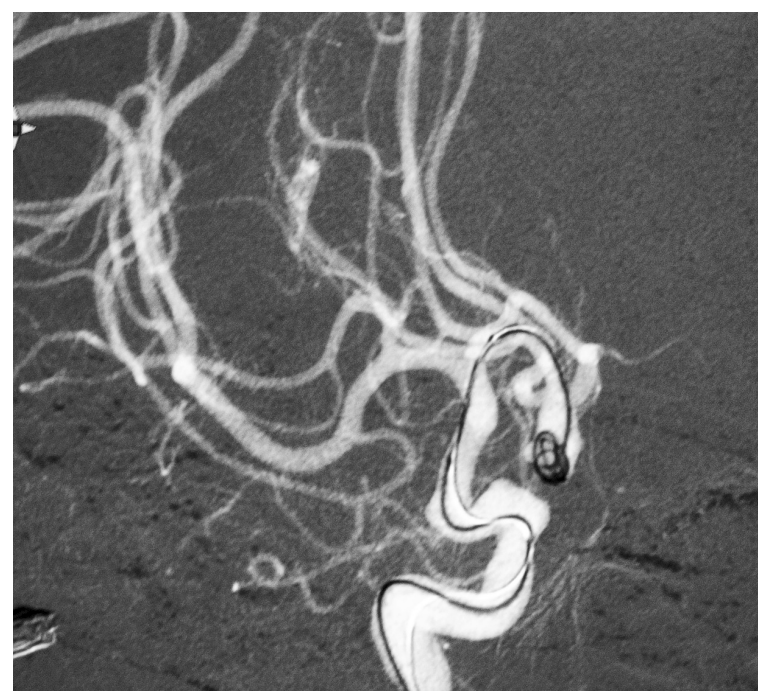

Figure 1.3: Angiogram of an endovascular coiling procedure.

is to limit flow into the aneurysm by redirecting the flow away, resulting in thrombosis within the sac. Preliminary clinical studies have demonstrated a success rate between $74 \%$ and $94 \%$ [17]. Despite the success rate of PEDs, there still remains a $3.2 \%$ risk of aneurysm rupture.

Overall the goal of these treatment options is to attempt to restore regular blood flow in the main artery. However, both pre- and post-operative treatment of intracranial aneurysms dramatically changes the hemodynamics. It is believed that the hemodynamic alternations and their interactions with the lumen of the arterial wall initiate pathological remodeling that leads to the post-surgical complications.

\subsection{Methods for monitoring neurovascular hemodynamic blood flow}

Currently, the gold standard for the evaluation of most neurovascular diseases is angiography. The most common angiography procedure (X-ray angiography) consists of subjecting the patient to low dose radiation, while contrast agent is injected into the vascular system. Other angiographic imaging techniques, such as digital subtraction angiography (DSA), computed tomography angiography (CTA), and magnetic resonance angiography (MRA) also require the injection of contrast agent and can only provide imaging resolution at $>100 \mu \mathrm{m}$. This is sufficient in determining the general geometric features of the aneurysm. These methods have also been proven useful for the detection of carotid stenosis and plaque ulceration [18]. In intracranial aneurysms, angiographic imaging can be utilized as a long-term monitoring tool; however, this imaging modality cannot provide detailed information about aneurysm wall microstructure or local hemodynamics. For patients presenting with unruptured cerebral aneurysms, another complication stems from the lack of well-established clinical criteria for determining whether to treat an unruptured aneurysm (and subject patients to treatment-related risks). This could also be due 
the limited quantitative capabilities to evaluate the hemodynamic changes with cerebral angiography.

Complications associated with angiography have been well documented during carotid or coronary imaging. The most common complication is acute renal failure, due to the iodine contrast agent, which occurs within 48 hours of radiographic imaging in $\sim 7 \%$ of patients [19]. In cerebral angiography, neurological complications can also occur [20]. Prospective analysis of $\sim 3000$ patients with complications had shown that $0.7 \%$ of patients resolved their complications within 24 hours (transient), $0.2 \%$ were within 24 to 7 days (reversible) and $0.5 \%$ were permanent [20]. A study which only involved patients with intracranial aneurysms receiving angiography demonstrated a $0.7 \%$ transient complication, $0.3 \%$ reversible complications and no reported permanent complications [21]. Finally, general complications that are inherent to the minimally invasive procedures are present. This includes infection, allergic reactions to medications, stroke, seizure, vascular injury, dissection, and pseudoaneurysm. During the stent placement, the main risk of the procedure is stroke, which exhibits a total risk of $\sim 4 \%$ ( $\sim 2 \%$ major and $\sim 2 \%$ minor stroke) and a $1 \%$ risk of myocardial infarction [22].

Transcranial Doppler (TCD) is a standard of care tool and has been utilized as a non-invasive imaging tool to assess cerebral hemodynamics, as well as the integrity of intracranial vasculature. Clinical application of TCD include detecting the presence of microemboli [23], assessing ischemia [24], stenosis, and vasospasm [25]. Monitoring can be performed during cerebrovascular procedures such as carotid endarterectomy (CEA) [26] or at the bedside. The key features that are used to evaluate these clinical indicators are flow velocities, change in frequency, and direction of flow. It has been shown that TCD monitoring reduced the number of non-disabling intraoperative strokes from $4.8 \%$ to $1.5 \%$ [27]. However, quantitative analysis through this imaging method is difficult, as it is largely influenced by the experience of the operator. In addition, measurements of cerebral blood flow velocities are limited to certain segments of intracranial vessels as typical TCD transducers are $1 \mathrm{MHz}$ (resolution $\approx 1.5 \mathrm{~mm}$ and flow sensitivity of $>1 \mathrm{~mm} / \mathrm{s}[28])$.

An invasive Doppler ultrasound technique has also been investigated during aneurysm clipping procedures. This procedure requires open craniotomies and involves specialized Doppler probes that are $\sim 1$ $\mathrm{mm}$ in diameter and operating at $16 \mathrm{MHz}$ (resolution $\approx 95 \mu \mathrm{m}$ and flow sensitivity of $\approx 1 \mathrm{~mm} / \mathrm{s}[28]$ ) are placed on the sac of the aneurysm [29]. Evaluation of the enveloped waveform, flow spectrum and acoustic signal were used as a metric to assess level of stenosis in the vessel and the amount of occlusion of the aneurysm sac. In general, regular cardiac flow patterns measured after placement of clip often signifies subsided SAH or no preceding bleeding; however, the absence of flow denotes total occlusion of the parent vessel. These indicators would determine if the clip would require repositioning. Studies have shown that $90 \%$ of vessels could be evaluated through this microvascular Doppler ultrasound technique [29]. Although the technique has shown clinical success, reports showed technological challenges. More specifically, flow from a residual aneurysm neck is below the resolution of the imaging modality, which, left undetected can lead to stroke or rebleeding.

At the current standard of care, the previously mentioned methods for monitoring of neurovascular hemodynamic blood flow are not optimized for monitoring and/or predicting successful treatment of neurovascular diseases. As a result, a higher resolution, low cost, lower risk, and real-time imaging system may be necessary to evaluate the state of the neurovasculature and its local hemodynamics. 
The following section provides an introduction to optical coherence tomography and Doppler optical coherence tomography imaging technology that aims to fill the gap between microscope and clinical methods, while providing the capability to measure hemodynamic changes in vivo and microstructural features.

\subsection{Optical Coherence Tomography}

Optical Coherence Tomography (OCT) is an emerging high resolution $(\sim 10 \mu \mathrm{m})$ imaging modality with initial clinical success in ophthalmology [30] and potential for significant clinical impact in other specialties such as cardiology [31], gastroenterology [32], and respirology [33]. These specialized fields are currently at different stages of technology development and clinical trials. OCT imaging systems have the capability to visualize subsurface tissue microstructural composition which is achieved through low coherence interferometry. It is common to compare OCT to ultrasound, as there are several similarities between the two modalities; however, instead of measuring backscattering pressure waves, OCT measures backscattering light through an interferometer. In the early stage of development, Fercher et al. [34] first employed this imaging technique on biological systems by evaluating the retinal pigment epithelium [35]. Fujimoto et al. [35] continued this work with their introduction of a fiber-optic based OCT imaging system. These initial systems were referred to as time-domain OCT (TD-OCT) and commonly consist of a Michelson interferometer configuration, as shown in Figure 1.4.

Light from a low coherence light source would be equally split, where $50 \%$ is directed to a reference arm mirror and the other $50 \%$ is directed to sample arm for tissue interrogation. The light is retroflected back, which produces constructive or destructive interference based on the optical path differences between the sample and reference arms. The intensity of the interference signal will be proportional to the local reflectivity of the tissue. As a result, by translating the reference mirror along the optical beam, depth information can be encoded with the local reflectivity, producing an axial scan or A-line. This spatial localization can be achieved by the use of a broadband source to produce interference patterns within a detectable range defined by its coherence length.

Sequential scanning of the A-line in the lateral direction produces a two-dimensional (2D) structural image known as a B-scan. For OCT imaging, both the axial and lateral resolution is decoupled. The axial resolution is inversely proportional to the bandwidth of the source and is defined by

$$
\delta z=l_{c}=\frac{2 \ln 2}{\pi \eta} \frac{\left(\lambda_{o}\right)^{2}}{\Delta \lambda},
$$

where $\Delta \lambda$ is the spectral bandwidth of the source, $\lambda_{o}$ is the center wavelength of the source, and $\eta$ is the refractive index of the tissue.

As for the lateral resolution, it is defined by the optics at the sample arm.

$$
\delta x=0.37 \frac{\lambda_{o}}{N A},
$$

where $N A$ is the numerical aperture. 


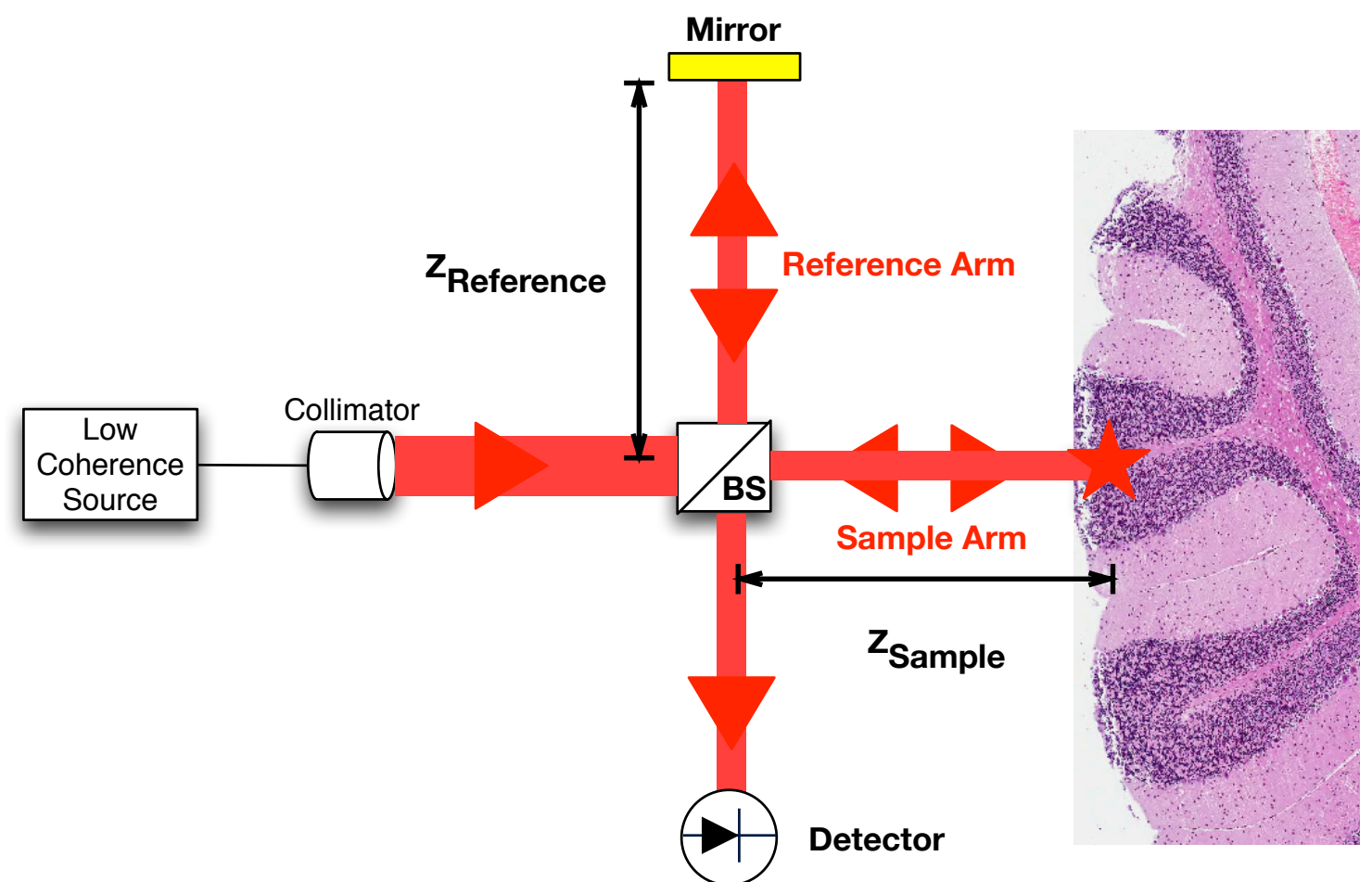

Figure 1.4: Simplified schematic of a TD-OCT system. Coupled light from a low coherence source is directed to a beam-splitter (BS). Half the light is split to a translating reference mirror and the remaining half is directed to a sample arm, which interrogates the tissue. The retroflected light is measured at the detector.

Advancements in OCT technology has progressed to a smaller form factor, improved signal to noise ratio (SNR), reduction in system complexity and higher A-scan scan rates compared to early TD-OCT systems. This recent generation of OCT systems, known as Fourier domain, is achieved through two methodologies: Spectral Domain OCT and Swept Source OCT (SS-OCT). In this work, we achieve high speed OCT imaging through SS-OCT imaging. The basic configuration of SS-OCT is similar to TDOCT. However, the distances for the reference and sample arms are held constant while the wavelength of the source is swept. The measured interference signal $(I(k))$ can be approximated as:

$$
I(k)=S(k)\left(1+2 \int_{0}^{\infty} r(z) \cos (2 k \eta z) d z\right),
$$

where $S(k)$ is the spectral intensity as a function of $k$ (the swept source), $r(z)$ the reflective profile of the sample, and $z$ is the depth coordinate [36].

Assuming that $r(z)$ is symmetrical with respect to $z$, we can replace $r(z)$ by a symmetric expansion 
$\bar{r}(z)$, which gives

$$
I(k)=S(k)\left(1+\int_{-\infty}^{\infty} \bar{r}(z) \cos (2 k n z) d z\right) .
$$

Taking the inverse Fourier transform of $I(k)$ we get

$$
F T^{-1}\{I(k)\}=F T^{-1}\{S(k)\} \otimes \delta(Z)+\frac{1}{2}\left(F T^{-1}\{S(k)\} \otimes \int_{-\infty}^{\infty} \bar{r}(z) \delta(Z \pm 2 n z) d z\right) .
$$

It can be seen that the symmetric reflective profile, as well as $r(z)$ can be gathered. The first term in equation 1.5 attributes to a DC $(z=0)$ term, while the second term is a cross correlation term that forms the A-line. This simplified equation neglects any autocorrelation terms which are associated with side lobes at $z=0$. The complex OCT signal can be then expressed as

$$
S=I+j Q
$$

where $I$ and $Q$ are the in-phase and quadrature of the decomposed inverse Fourier transform of $I(k)$.

To form a $2 \mathrm{D}$ (B-mode) structural OCT image, $S$, the magnitude is taken, $S=\sqrt{I^{2}+Q^{2}}$. The averaged B-Mode structural OCT image can be achieved by

$$
\left\langle S^{2}\right\rangle=\frac{1}{M N} \sum_{m=1}^{M} \sum_{n=1}^{N}\left[I_{m, n}^{2}+Q_{m, n}^{2}\right]
$$

where $M$ represents the depth window length, $N$ represents the lateral window length, $m$ and $n$ are the indices for the depth and lateral direction.

Figure 1.5 depicts a structural OCT image of resected intracranial aneurysm and its corresponding histology. Similarities between the gold standard histology and the structural OCT image can be observed. In-depth analysis and correlation of intracranial aneurysm will be presented in Chapter 5 .

a)

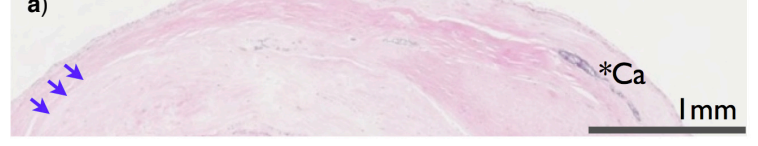

b)

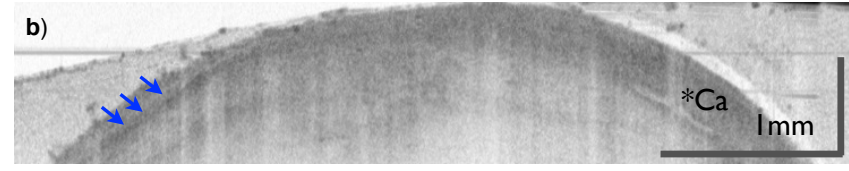

Figure 1.5: (a) The hematoxylin and eosin $(\mathrm{H} \& \mathrm{E})$ stained histologic of a human intracranial aneurysm. Layers (arrows) and calcium deposits $\left({ }^{*} \mathrm{Ca}\right.$ ) can be observed. (b) The corresponding structural OCT image. Scale bar: $1 \mathrm{~mm}$.

\subsection{Doppler Optical Coherence Tomography}

Similar to ultrasound, OCT has the capability to provide functional information. Although there are considerable differences between OCT and ultrasound (such as physical interaction, wavelength, and 
velocity), there are also similarities from the signal processing prospective. When two successive A-lines are acquired at a stationary location within a short period of time, the signals become shifted in time as a result of the scatterers motion. Figure 1.6 depicts this received signal over multiple acquisitions stacked on top of each other, as the single scatterer is moving within the optical beam. At a depth of interest (dashed line), samples are equally taken and traced out in Figure 1.6(b), which is known as the slow time axis signal. This slow time axis signal can be used to estimate the frequency shift between the successive A-lines, commonly referred to as the Doppler frequency shift $\left(f_{D}\right)$. The frequency relationship between the slow time axis signal and the measured phase shift is given by
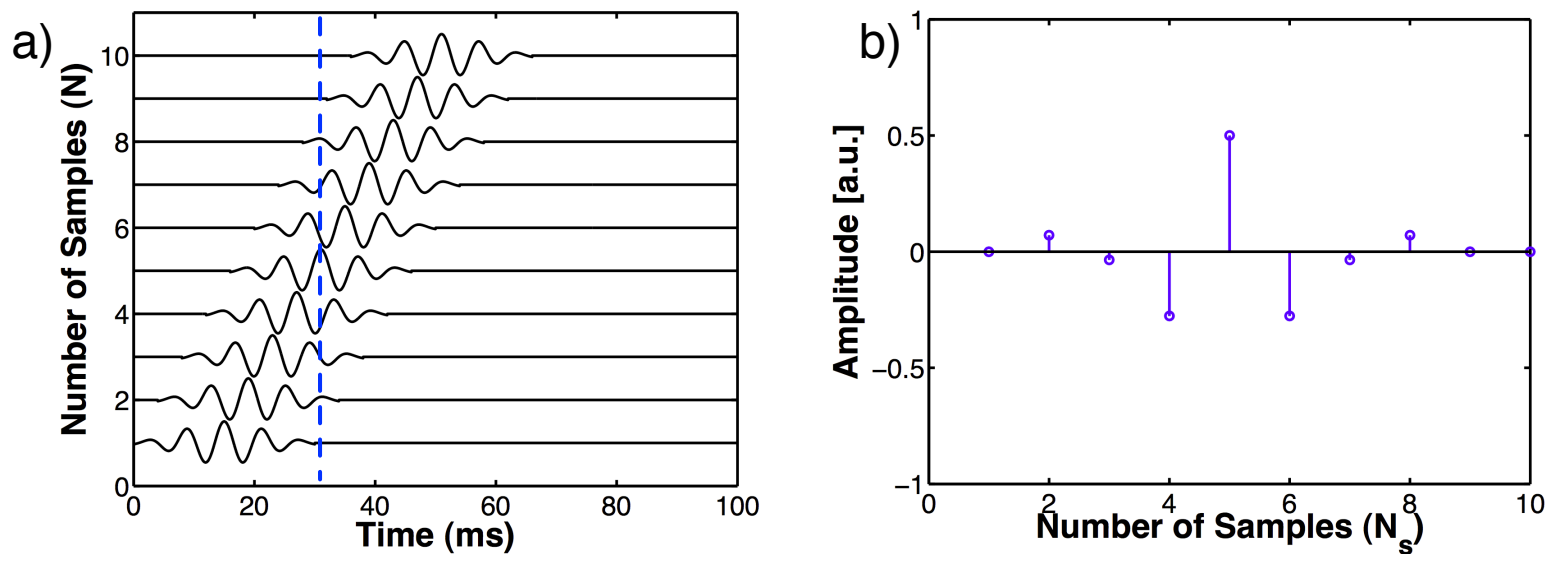

Figure 1.6: (a) A representation of the detected OCT A-scan from a single scatterer moving through the optical beam. (b) Sampled dataset of the OCT A-scan in slow time axis.

$$
f_{D}=\Omega f_{a},
$$

where $\Omega$ is the phase shift and $f_{a}$ is the axial scanning rate.

It should be noted that this is not the classic Doppler effect of measuring the frequency change from the receiver relative to the source frequency. Instead, the measurement of the Doppler frequency shift has been established to be an artefact in a pulsed wave system in ultrasound. One approach to estimate the Doppler frequency shift is to evaluate the local phase change by comparing adjacent depth scans. The most common method to measure Doppler frequency shifts is the utilization of an autocorrelation technique called the Kasai velocity estimator, which is given by:

$$
f_{D}=\frac{f_{a}}{2 \pi} \arctan \left\{\frac{\frac{1}{M(N-1)} \sum_{m=1}^{M} \sum_{n=1}^{N-1}\left(I_{m, n+1} Q_{m, n}-Q_{m, n+1} I_{m, n}\right)}{\frac{1}{M(N-1)} \sum_{m=1}^{M} \sum_{n=1}^{N-1}\left(Q_{m, n+1} Q_{m, n}+I_{m, n+1} I_{m, n}\right)}\right\}=\frac{f_{a}}{2 \pi} \arctan \left\{\frac{\langle Y\rangle}{\langle X\rangle}\right\} .
$$

The derivation of this equation in one of the axises can be found in Appendix 1. These frequency shifts are then used to form a Doppler OCT (DOCT) image. Figure 1.7 shows the structural OCT 
image of a patient with HHT. As previously mentioned, HHT has been associated with the formation of an intracranial aneurysm. HHT is a rare genetically heterogeneous vascular dysplasia characterized by abnormal vasculature. As a result, Doppler frequency shifts would be localized at the blood vessels seen in the corresponding DOCT image (Figure 1.7).

a)

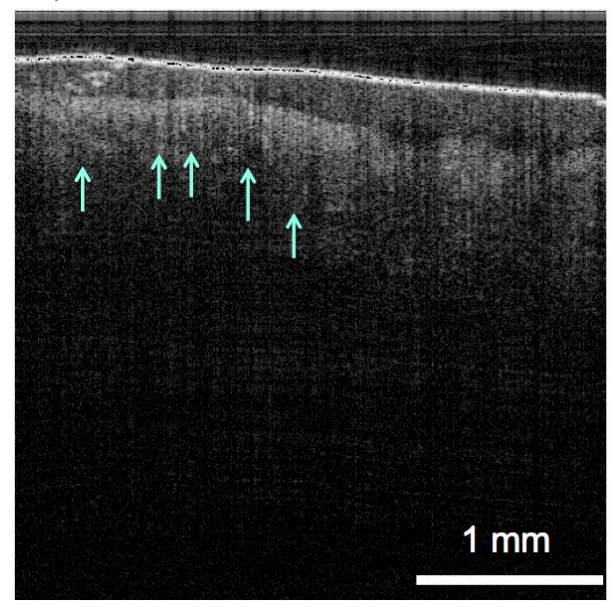

b)

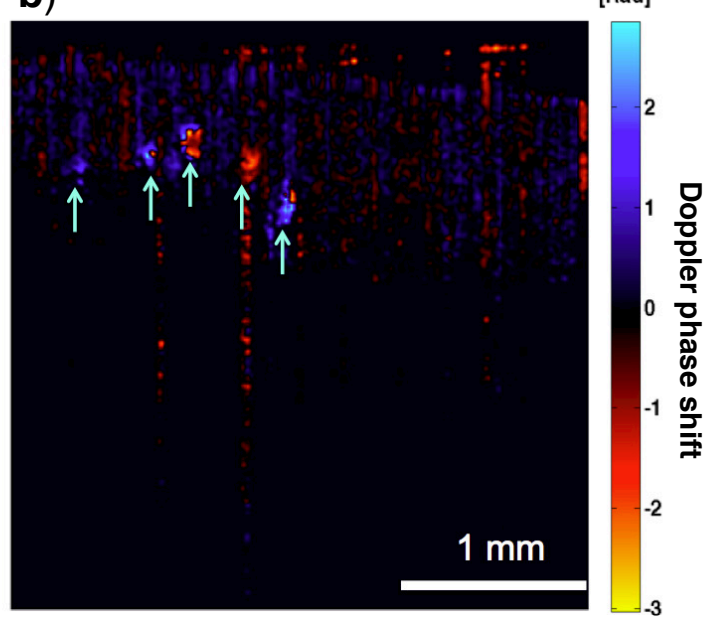

Figure 1.7: (a) Structural OCT image of a HHT patients fingertip with abnormal vasculature. (b) The corresponding DOCT image shows blood flowing through superficial micro vessels (arrows). Scale bar: $1 \mathrm{~mm}$

As blood moves through the optical beam at an angle $\theta$ (Doppler angle), the measured mean velocity $(<V>)$ can be related to the phase shift by,

$$
<V>=\frac{\lambda_{o} \Omega f_{a}}{4 \eta \cos (\theta)} .
$$

This method of analysis provides a quantifiable measure of the blood vessel and is commonly utilized in clinical ultrasound. Yang et al. [2] had evaluated this autocorrelation based estimator technique and had reported a relationship between the set velocity (without Doppler angle correction) and phase variance. This was depicted in Figure 1.8 in terms of normalized standard deviation. It was speculated that the trend had followed an inverted Gaussian; however no formal proof was presented. During high flow estimation, several contour rings had occurred due to aliasing phase wrapping. The minimum detectable velocity was reported to be $+/-2 \mu \mathrm{m} / \mathrm{s}$ and the maximum velocity was $+/-1.9 \mathrm{~mm} / \mathrm{s}$ without aliasing [2].

There have been a significant amount of studies that have demonstrated the clinical advantage for the use of DOCT. However, there are practical issues that need to be overcome. One of the major factors is the physiological or bulk tissue motion, which can be orders of magnitude larger than blood flow velocities [37]. Yang et al. [37] have demonstrated the ability to remove a majority of these artefacts 


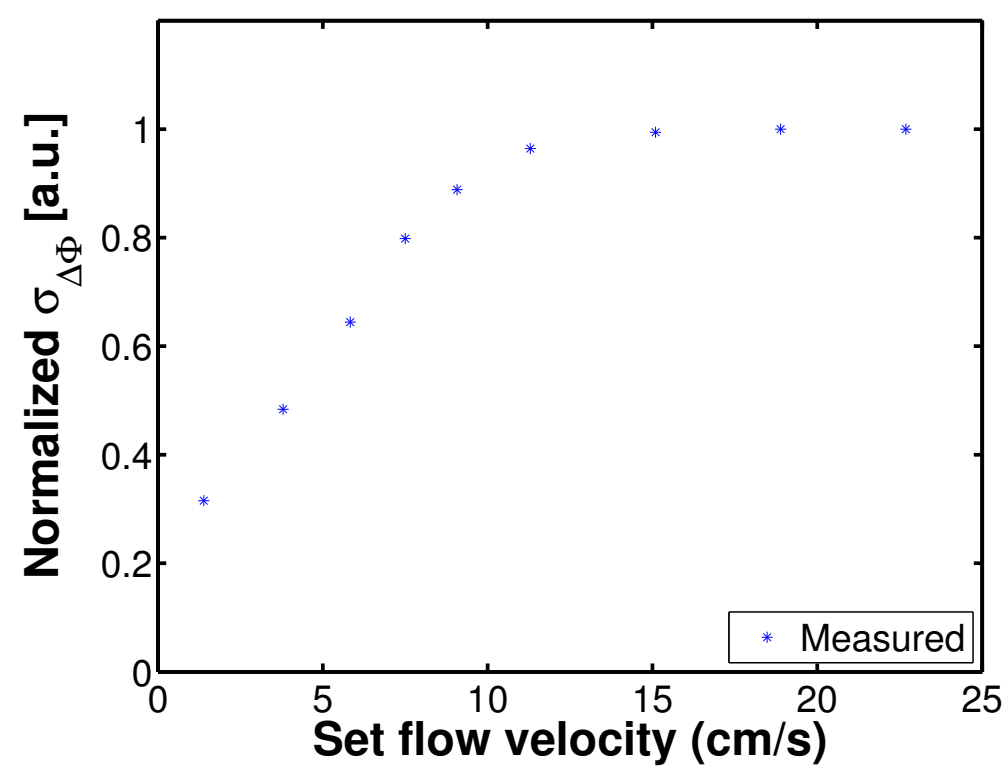

Figure 1.8: Regenerated dataset of normalized standard deviation versus flow velocity (without Doppler angle correction). Each data point is the mean of 25,000 pixels [2].

through a histogram-based velocity segmentation method. Furthermore, during B-scanning to form 2D OCT images, lateral displacement between each A-line leads to increase in Doppler noise [2]. Reduction of this effect can generally be achieved through dense or oversampling scans, but at the cost of a lowered frame rate.

Another important factor that contributes to Doppler noise is SNR. It has been established that the flow sensitivity estimates are fundamentally limited by phase stability of the OCT system [38]. This phase stability is limited by the OCT system noise floor, which has been shown to be related to the SNR. This SNR limited Doppler noise is considered to be the limiting phase shift that can be measured between two A-scans. In a scenario where the SNR is good (signal>> noise), the standard deviation of phase shifts can be written as [38],

$$
\sigma_{\Delta \phi}=\frac{1}{S N R^{1 / 2}}
$$

\subsection{Thesis and Scientific Contributions}

The previously described method of reducing Doppler noise has shown success in the bulk laboratory bench-top scanning environment. However, it is often difficult to translate these successes to the clinical setting. In order for DOCT to demonstrate clinical utility, fiber-optic devices designed for seamless integration in the physician's workflow must replace the laboratory bench-top scanning system. This introduces many technological and practical hurdles, which would require an alternative approach from the laboratory environment. 
Inspired by Kasai et al. [39] and Yang et al. [2], we proposed in this thesis an intravascular DOCT imaging method to visualize hemodynamic changes during neurovascular procedures. Prior to this $\mathrm{PhD}$ work, limited developments of intravascular DOCT imaging were reported. Although the initial feasibility study utilized similar techniques to estimate flow in blood vessel, the major contribution of this $\mathrm{PhD}$ work was the development a novel signal processing technique known as split spectrum Doppler optical coherence tomography (ssDOCT) that suppresses Doppler noise sources. ssDOCT consists of splitting or separating the interferometric signal into multiple bands which then utilized to estimate the flow velocity. Intravascular Doppler system characterization methods were designed to compare conventional DOCT and ssDOCT techniques. ssDOCT exhibited an improved Doppler noise suppressed over the conventional DOCT technique and allowed for higher sensitivity to low velocity measurements. We then demonstrated, to the best of our knowledge, the first in vivo Doppler imaging of carotid stent placement in pigs with ssDOCT. Computational fluid dynamic (CFD) models were also utilized to provide further understanding of the local hemodynamics and showed similarities to the ssDOCT images. It should be noted that the ability to evaluate the hemodynamics change due to malapposition of stents could provide insight into the cause of post-procedural complications such as stroke. The interaction between blood flow and stent struts on the lumen has been observed externally through flow phantoms and patient specific CFD models. As a result, intravascular ssDOCT imaging has the potential to monitor human carotid stenting.

The knowledge that was gained from the carotid stenting study was then applied to intravascular ssDOCT imaging of intracranial aneurysms. Both hemodynamic and microstructural changes are believed to be key factors in the rupture of intracranial aneurysms. This resulted in the demonstration of intravascular ssDOCT imaging to evaluate hemodynamic events using ideal and fabricated patientspecific intracranial flow phantoms. Certain flow profiles have been known to promote thrombogenesis which could result in the rupture of the intracranial aneurysm. The deposition of thrombi as well as key morphological identifiers that are associated with vascular remodeling can be observed in the structural OCT images. As a result, we presented, to the best of our knowledge, the largest human ex vivo study of intracranial aneurysms with a bench-top structural OCT system. Tissue handling protocols were developed to accurately correlate histological sections with structural OCT images. OCT imaging showed the capability to resolve several morphological identifiers. However, the evaluation of the tissue would require a trained OCT technician. To provide pathologists the ability to associate the structural OCT visualization of the tissue, we developed an image segmentation guided optical attenuation imaging technique. This OCT optical attenuation (OCT-OA) imaging provided enhanced contrast to the traditional OCT image. Both the structural OCT imaging and OCT-OA methods could be utilized in an intravascular OCT catheter setting. Overall, we show that intravascular DOCT imaging has the potential to evaluate both the hemodynamic and microstructural changes in neurovascular diseases. The contribution that had disseminated from this $\mathrm{PhD}$ could provide a role to translate intravascular DOCT imaging technology to the surgical operating room. 


\subsection{Outline}

The key contributions that were described in the previous section are presented in their respective chapters, and is summarized in Figure 1.9. Chapter 2 describes the development and characterization of an intravascular DOCT system and had demonstrated several Doppler artefacts. This resulted in the implementation and optimization of the novel ssDOCT technique. Phantom flow models and in vivo porcine models were utilized to demonstrate the improved Doppler noise suppression over the conventional DOCT method. In Chapter 3, we present our in vivo animal experiments using the developed ssDOCT system and algorithm. More specifically, we demonstrated the evaluation of carotid stent apposition and its hemodynamic effect. CFD modeling was employed to provide additional understanding of the flow mechanisms. Chapter 4 further demonstrates the ssDOCT in ideal and patient specific intracranial aneurysm flow models. The other critical factor in the assessment of intracranial aneurysms consisted of the microstructural composition. Chapter 5 describes the correlation between OCT and histology of resected human intravascular aneurysms. Chapter 6 concludes this work with a summary and the future directions for further improvement and increasing clinical utility of intravascular DOCT imaging.

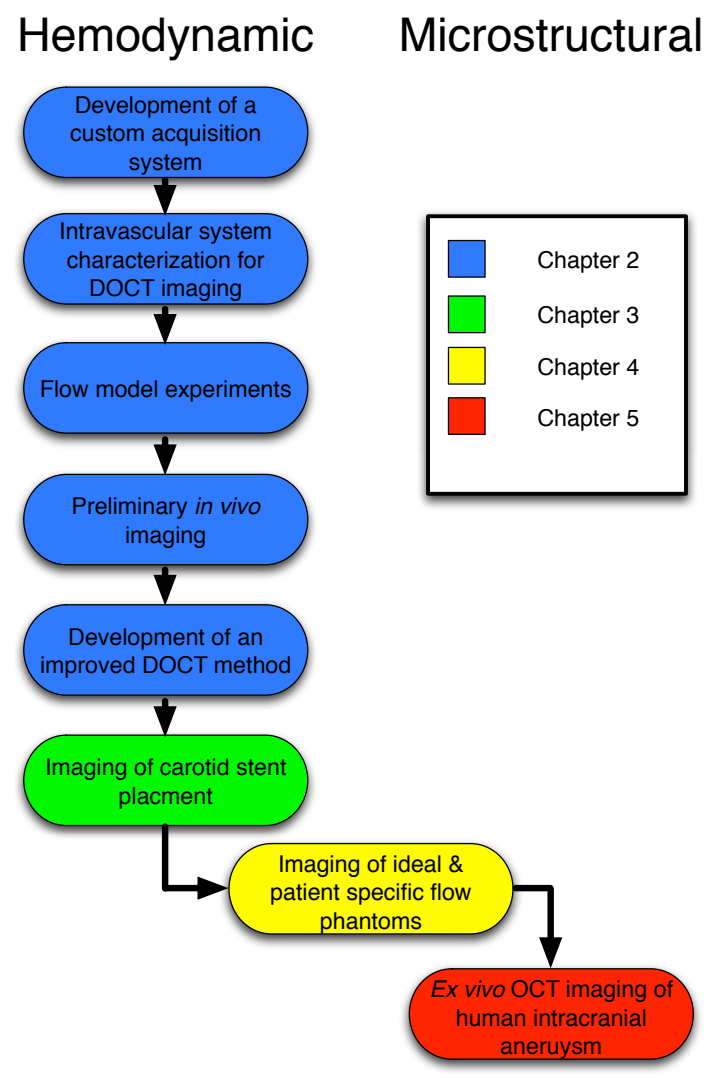

Figure 1.9: Block diagram of outline. 


\section{Chapter 2}

\section{Intravascular split spectrum Doppler optical coherence tomography}

As previously mentioned, morphological changes are directly linked to the interaction of blood and the vessel wall. As a result, functional imaging could serve as a metric to predict hemodynamic outcomes. The following chapter describes the development of split spectrum Doppler OCT in a rotary catheter regime for in vivo imaging.

The work presented in this chapter resulted in two journal publications. In our initial publication, the feasibility of DOCT imaging and issues in adaptation of the rotary catheter regime were outlined and described.

In the second publication, the split spectrum Doppler OCT (ssDOCT) technique was described, Doppler noise flow characterization was measured, flow phantom measurements were performed and in vivo measurements were validated against the computational fluid dynamic simulations (CFD).

1. Vuong, B., Lee, A.M.D., Luk, T.W.H., Sun, C., Lam, S., Lane, P., Yang, V.X.D. (2014) High speed, wide velocity dynamic range Doppler optical coherence tomography (Part IV): split spectrum processing in rotary catheter probes. Optics Express. 22:7399-7415 (PhD Work).

2. Sun, C., Nolte, F., Vuong, B., Lee, K.K.C, Standish, B.A., Courtney, B., Standish, B.A., Marotta, T.R., A., Mariampillai, A., Yang, V.X.D. (2012) In vivo feasibility of endovascular Doppler optical coherence tomography. Biomedical Optics Express 3:2600-2610 (PhD Work).

\subsection{Intravascular Doppler optical coherence tomography}

Prior to this work, intravascular ultrasound (IVUS) has been reported to evaluate blood flow in the coronary arteries [40]. Current IVUS catheters have a resolution of 70 to $200 \mu \mathrm{m}$ (45 MHz to $20 \mathrm{MHz}$ ) with a penetration depth $>5 \mathrm{~mm}[41,42]$. However, IVUS has difficulty delineating between thrombus 
formations and fibrotic plaques [43]. These catheters consist of a rotating single-element and can quantify blood flow using decorrelation-based methods [44].

Intravascular OCT has been used in vivo to image normal coronary artery (with or without mild intimal thickening), coronary atherosclerotic plaques (with or without calcification), and thrombosis or dissection pathologies. Li et al. [45] first presented a prototype DOCT rotary catheter with an accurate estimation of flow profile in phantoms. This prototype rotary catheter provided a circumferentially scanning catheter probe, resulting in visualization of cross-sectional flow contours. However, prior to this $\mathrm{PhD}$ work, limited in vivo velocity estimation with rotary catheter had emerged since the initial work. Linear translating needle-based probes have been investigated during in vivo estimation of velocity of gastrointestinal tracts $[46,47]$ and monitoring of microvasculature during therapy. It was observed that mechanical vibrations due to rapid linear scanning of the probe and substantial motions were key factors in the formation of Doppler noise. These technological hurdles would also be present in rotary catheters, as well as other challenges.

\subsubsection{Intravascular Doppler optical coherence tomography system}

The current commercially available intravascular OCT system (C7-XR by LightLab Imaging, St. Jude Medical Inc., USA) is a rotary catheter based imaging system that is food and drug administration (FDA) approved for human coronary imaging. This intravascular swept source OCT system was utilized for all animal model-imaging experiments. The intravascular swept source OCT system consisted of a spectral bandwidth of $110 \mathrm{~nm}$, centered at $1310 \mathrm{~nm}$, at an A-line rate of $50.4 \mathrm{kHz}$. The default scanning mode for imaging within an artery is a spiral scan consisting of a rotating radial scan coupled with a retraction (pullback) of the imaging probe, with each motion being independently controlled. The image data set consists of a sequential set of longitudinally spaced, cross-sectional image frames often referred to as a helical scan. This system has an imaging frame rate to $\sim 100$ frames per second during helical scan. During DOCT acquisition, 2,500 A-lines were acquired at a frame rate of $\sim 20$ frames per second.

It should be noted that the C7-XR clinical software does not estimate velocity or produce DOCT images. To estimate Doppler frequency shift, the interferogram between adjacent depth scans is required. This resulted in the construction of a custom acquisition system to capture the interference OCT signal from the C7-XR [48]. The acquisition system consisted of a high-speed data acquisition card (ATS9350, AlazarTech, Canada), which was connected to the k-clock, A-scan trigger, and the detected interference signals of the C7-XR (Figure 2.1). Data collected from the system was resampled to linear k-space by the k-clock, which allowed for Doppler frequencies measurements. One of the practice issues with swept source based OCT imaging is that data acquisition cards (DAQ) sample the detected signal linear in time. Recalibration from time to k-space is necessary to resolve the depth information. This resampled interference OCT signal is then directly stored onto a high speed solid state drive (RevoDrive $3 \mathrm{X} 2$, OCZ, USA). A graphical processing unit (GeForce GTX 460 1GB, NVIDIA, USA) computes the inverse fast Fourier transform for each detected OCT signal to provide real-time structural OCT feedback. DOCT images were post-processed from the store datafile.

The sample arm of the C7-XR system was connected to a disposable OCT rotary catheter (Figure 


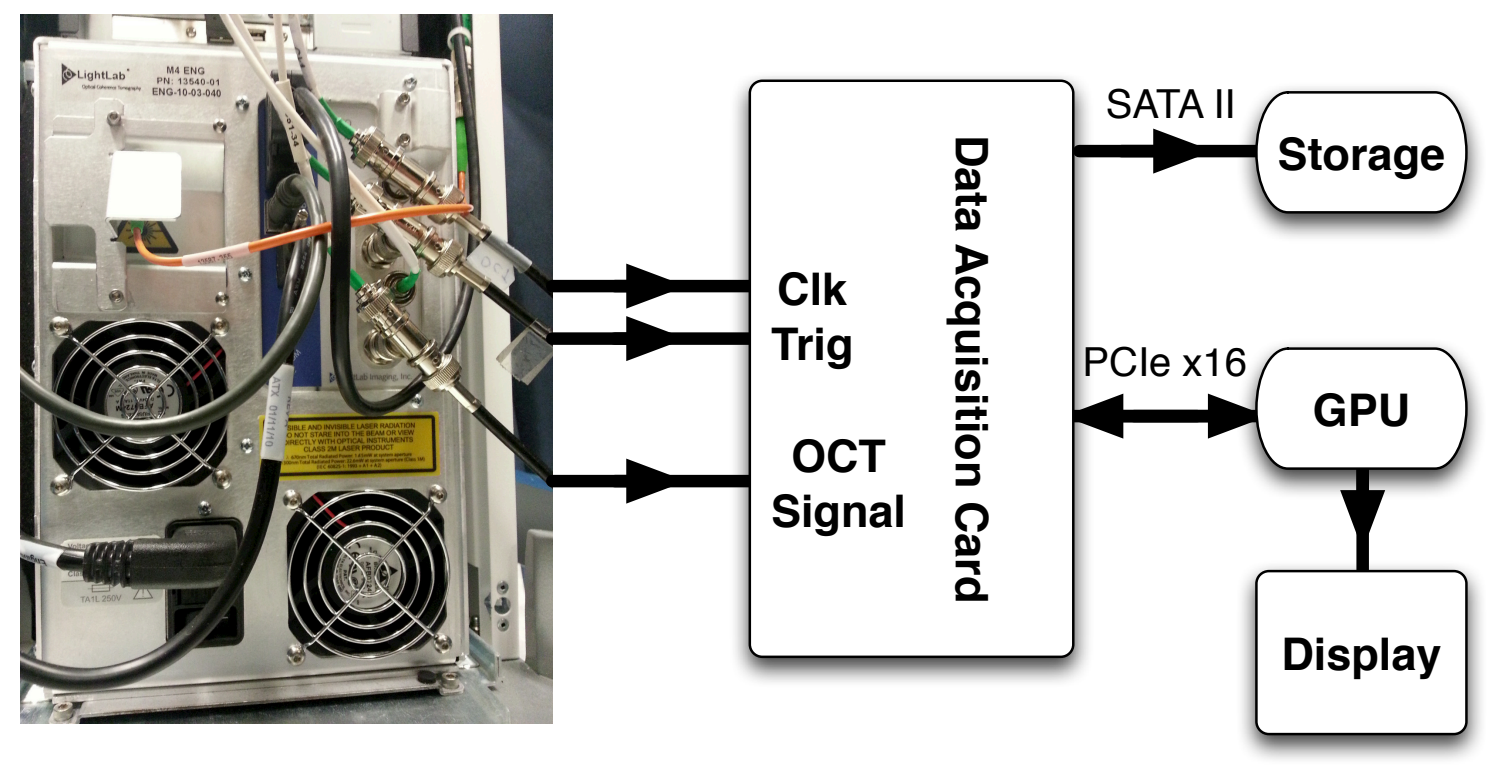

Figure 2.1: Schematic of the the custom data acquisition system. The interference OCT signal, A-line trigger (Trig) and k-clock (Clk) are coupled out to a PC based data acquisition card. The interference OCT signal is recorded on a high speed storage drive, while the graphical processing unit (GPU) performs inverse fast Fourier transforms to provide real time structural OCT images to a display.

2.2(a) and Figure 2.2(b); C7 Dragonfly, St. Jude Medical Inc. St. Paul, Minnesota, USA). The typical OCT rotary catheter consists of a single-mode optical fiber at the proximal end and is fused to a graded index (GRIN) fiber followed by a microprism to direct light perpendicular to the direction of flow. The angle between the axis of the catheter and incident beam was measured to be $\sim 70$ degrees in air with a spot size of $25 \mu \mathrm{m}$ and working distance of $\sim 1.5 \mathrm{~mm}$. It was observed that there was a $\sim 80 \%$ beam overlap between adjacent A-line scans at a radial distance of $\sim 4 \mathrm{~mm}$, which was sufficient to calculate Doppler phase shift [3].

The optical fiber probe is attached to a torsion cable to improve structural integrity while providing rotation for circumferential scans. This inner component of the catheter is decoupled from an outer catheter sheath, where it is kept stationary. During volumetric imaging, the inner component rotates and is pulled back to a maximum distance of $\sim 50 \mathrm{~mm}$.

\subsubsection{System characterization for Doppler imaging}

A major contributor to Doppler phase noise is motion artefacts. During in vivo navigation of the OCT catheter to the vessel of interest, bending and twisting of the catheter sheath would occur. While high-speed circumferential scans were being acquired, the coupled tension from the sheath induced vibrations. This introduced undesired relative motion in both radial and longitudinal direction. In severe occurrences, the movement of the catheter sheath and variations in rotational speed produced artifacts in the structural imaging known as non-uniform rotational distortion (NURD) [49, 50]. 
a)

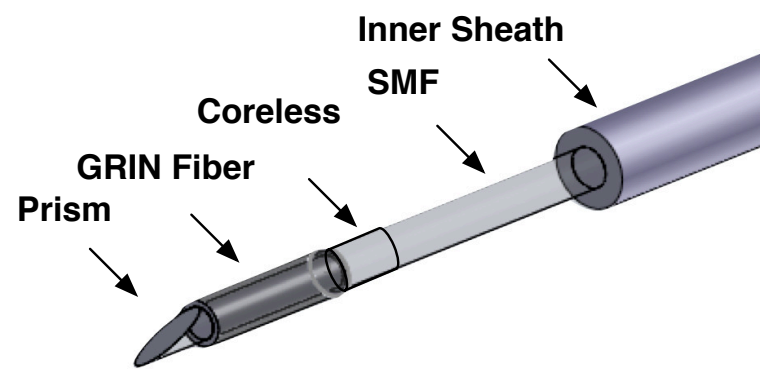

b)

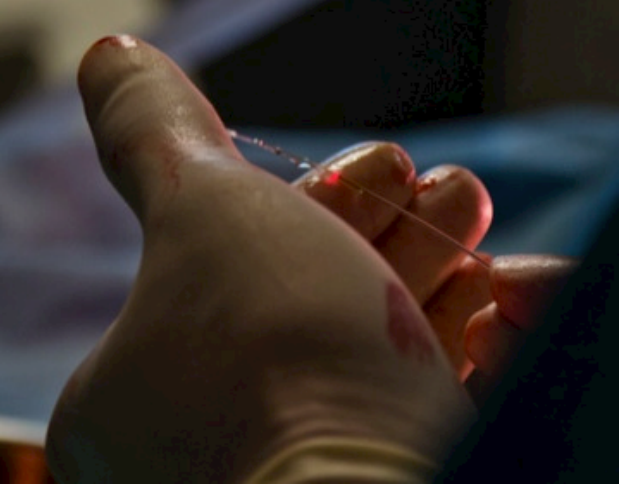

Figure 2.2: (a) Schematic of the C7 Dragonfly. The distal end of the catheter consists of a microprism fused to a GRIN fiber, coreless fiber, and single mode fiber (SMF). All optics were encased in an inner catheter sheath. (b) A photograph of the C7 Dragonfly catheter.

It has been suggested that Doppler shift estimation is more sensitive to these relative motions, and significant artefacts can occur without the presence of structural distortions in the image $[3,51]$. These factors would limit the minimum detectable velocities of the system. As a result, characterization of the intravascular system was necessary to determine the velocity sensitivity.

The sensitivity of measuring flow is fundamentally dependant on the phase stability of the given OCT system [38]. The measurement of the phase stability of the intravascular system was achieved by introducing a reflector in the sample arm [4]. An accumulation of phase shifts was measured at the stationary sample reflector. The standard deviation of the phase was found to be $0.01 \mathrm{rad}$ and was considered to be the phase stability or the phase noise floor of the system [4].
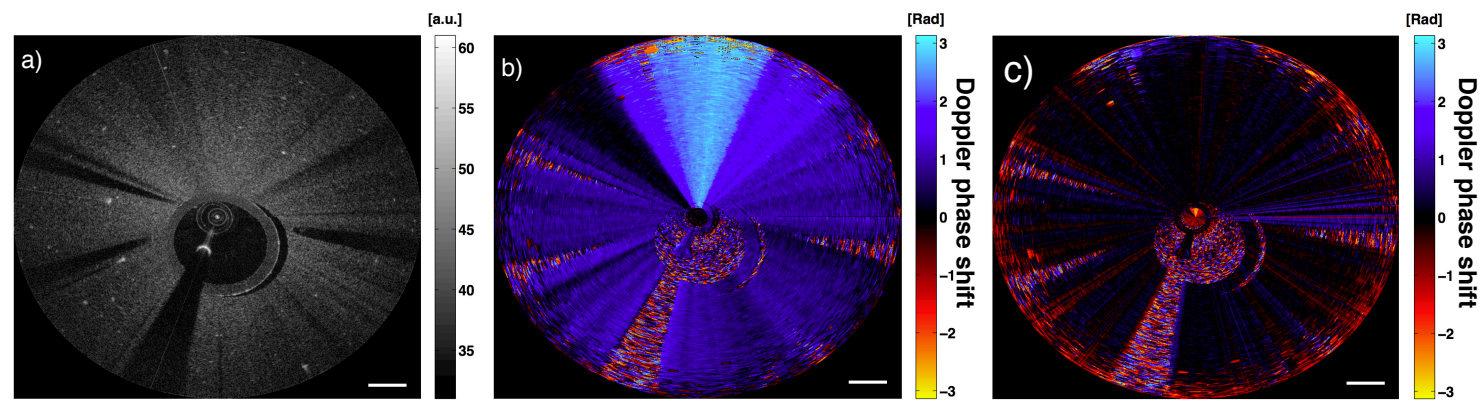

Figure 2.3: (a) OCT image of a stationary phantom. (b) Doppler OCT image without bulk motion compensation. (c) Doppler OCT image with bulk motion compensation. Scale bar $1 \mathrm{~mm}$.

To determine the minimum detectable velocity during a circumferential scan, a titanium dioxide $\left(\mathrm{TiO}_{2}\right)$ gelatin phantom was fabricated. This type of phantom is commonly used to model the optical properties of tissue. To guide the catheter, a clear plastic tube with $\sim 3 \mathrm{~mm}$ outer diameter was embedded in the gelatin phantom. Once the OCT catheter was guided to the location of interest, cross sectional images were acquired (Figure 2.3). Utilizing Equation 1.9 in Chapter 1 with a Kasai autocorrelation 
window of $2 \times 10(\mathrm{M} \times \mathrm{N})$, the corresponding Doppler imaging is shown in Figure 2.3(b). This initial Kasai autocorrelation window of 2 by 10 was selected based on the improved velocity detection sensitivity in the controlled phantom flow experiment in the next section. A constant radial phase shift from the outer catheter sheath was observed in the $3 \mathrm{~mm}$ insertion tube and phantom. The constant radial phase shift artefact observed in the intravascular DOCT image may be due to the mechanical vibrations [3]. Any Doppler shift within this artefact would be completely masked. As a result, the characterization of the Doppler noise floor of the intravascular OCT system was evaluated after compensation.

Previous work has shown vertical bands as artefacts due to bulk motion in bench-top DOCT imaging systems, and can be compensated by a histogram-based velocity segmentation method (Chapter 1). This method was employed to suppress the Doppler noise artefact in the DOCT image. After compensation, it can be seen that the artefact was removed. Five regions in the phantom were utilized to calculate the Doppler noise floor. This characterization technique is similar to the single reflector measurement; an assumed stationary phantom was utilized to measure phase stability at 2500 A-lines per frame and 500 A-lines per frame as a function of radial distance, which in turn is a function of SNR. The two A-line rates demonstrate different sampling in the angular direction. Furthermore, it has been well established in both ultrasound and OCT that the phase stability is inversely proportional to SNR [38].

Further intravascular catheterization consisted of the measurement of Doppler shifts in the internal reflection of the catheter (Figure 2.4(a)) and comparing this to the Doppler shifts in the outer catheter sheath (Figure2.4(b)). The Doppler shift of the catheter sheath was significantly larger $\left(S T D_{c} \approx 3 \mathrm{rad}\right)$ than the Doppler shift exhibited by the internal reflection $\left(S T D_{i r} \approx 0.02 \mathrm{rad}\right)[3]$.

\subsubsection{Phantom flow measurements}

The estimation of velocity and depth penetration in DOCT is correlated to the optical attenuation and scattering from the moving particle. More specifically, multiple scattering (often occurring in blood) results in the decrease in SNR with depth. As a result, low SNR regions may lead to unresolvable Doppler shifts. In order to improve the OCT catheter's depth penetration, numerous studies have suggested the use of a saline injection for visualization of the lumen $[43,45,52]$. However, the lack of scatterers in pure saline would not produce measurable Doppler shifts. To measure Doppler shifts in arterial vessels, a mixture of saline \& blood was utilized as a contrast agent. An infusion pump injected $50 \mathrm{ml} / \mathrm{hr}(\sim 3.5 \mathrm{~mm} / \mathrm{s}$ peak velocity) of the mixture into a phantom flow model. It was found that a mixture of blood ( $1 \%$ to $1.5 \%$ by volume) in saline was the optimal concentration to measure Doppler shifts, while maintaining visualization of the arterial wall. The structural OCT image is shown in Figure 2.4(c). Figure 2.4(c) and Figure 2.4(d) demonstrated the corresponding uncompensated DOCT image and compensated averaged DOCT image, respectively. The measured peak average phase shift was 0.4 $\mathrm{rad}$, which corresponds to a velocity of $4 \mathrm{~mm} / \mathrm{s}$ and was comparable to the expected velocity. However, it was observed that a blood in saline mixture greater than $\sim 2.5 \%$ demonstrated increase in Doppler noise in the unaveraged DOCT images (Figure 2.5). 

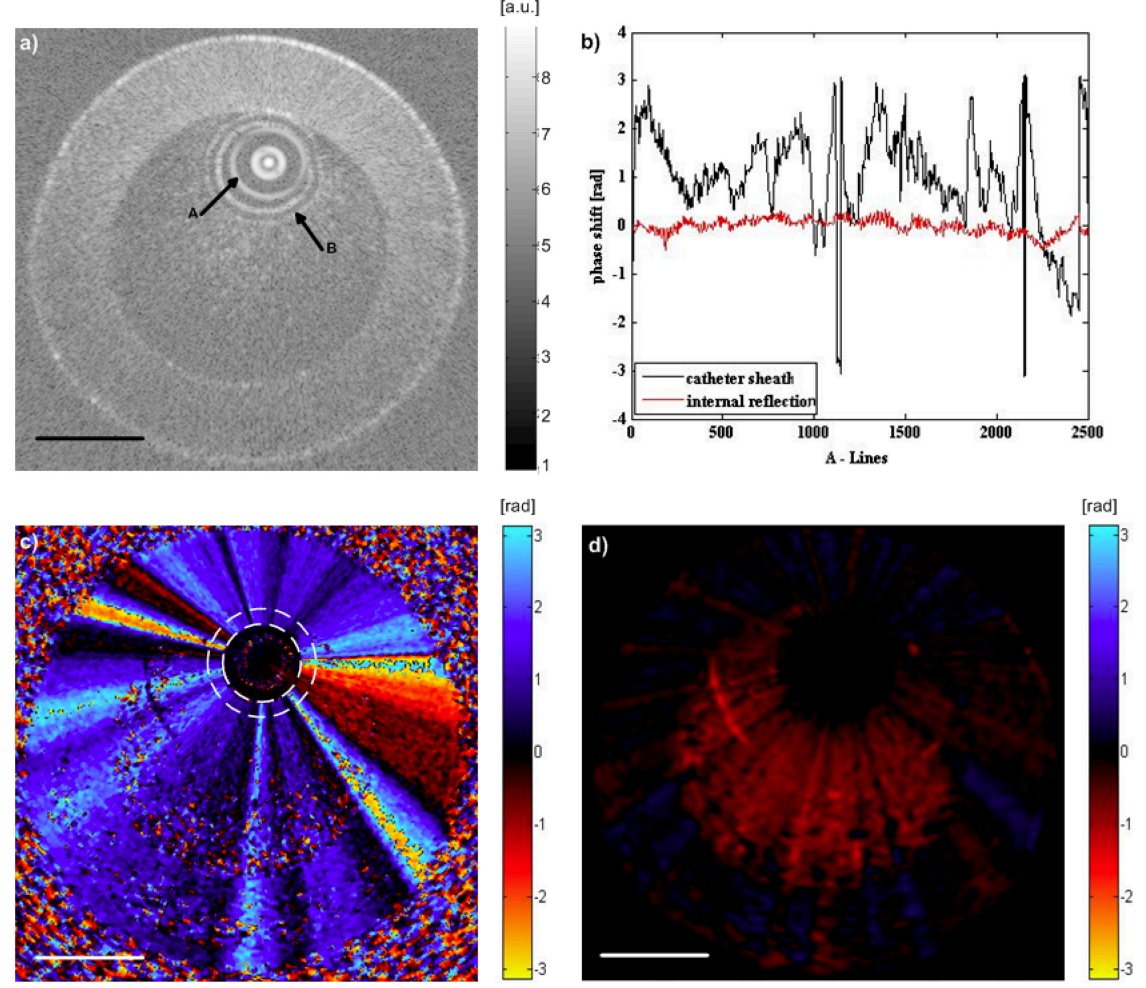

Figure 2.4: Imaging of a slow flow phantom. (a) Structural image of 1.5\% blood in saline mixture within the tube, where arrow A indicates the internal reflection of the imaging optics, and arrow B indicates the outer surface of the imaging catheter sheath. (b) Phase shift obtained from the internal reflection indicated by arrow A and catheter sheath indicated by arrow B in (a). The phase shift of the catheter sheath is the median of data along the catheter thickness. (c) Doppler shift image of the slow flow phantom. The dashed ring indicates the sheath of the imaging catheter, with phase shift induced by NURD in a radially constant manner throughout the image. (d) Doppler image after suppression of the motion artifact, which displays the phase shift induced by the slow flow inside the tube phantom. Scale bars $=1 \mathrm{~mm}[3]$. (Reprinted with permission of the Optical Society of America)

\subsubsection{In vivo imaging of porcine animal model}

Porcine is thought to be a suitable experimental animal model for the human common carotid bifurcation. The external iliac diameter $(6-7 \mathrm{~mm})$ in the lower weight swine is similar to the known human common carotid artery [53]. The greatest similarity was found between the diametrical ratios of the human internal carotid artery to common carotid artery (ICA / CCA mean 0.63) and the swine profunda to external iliac (profunda / Iliac mean 0.68). This suggest that the swine iliac bifurcation can be used as a comparable anatomy to the human carotid bifurcation [53]. All procedures were carried out with institutional approval at the St. Michaels Hospital, Toronto, Canada and Sunnybrook Health Science Centre, Toronto, Canada.

The surgical procedure generally involved the following: a small catheter $(\sim 5 \mathrm{~F})$ is first inserted by femoral puncture and then navigated to the common carotid artery. After angiograms are completed to 

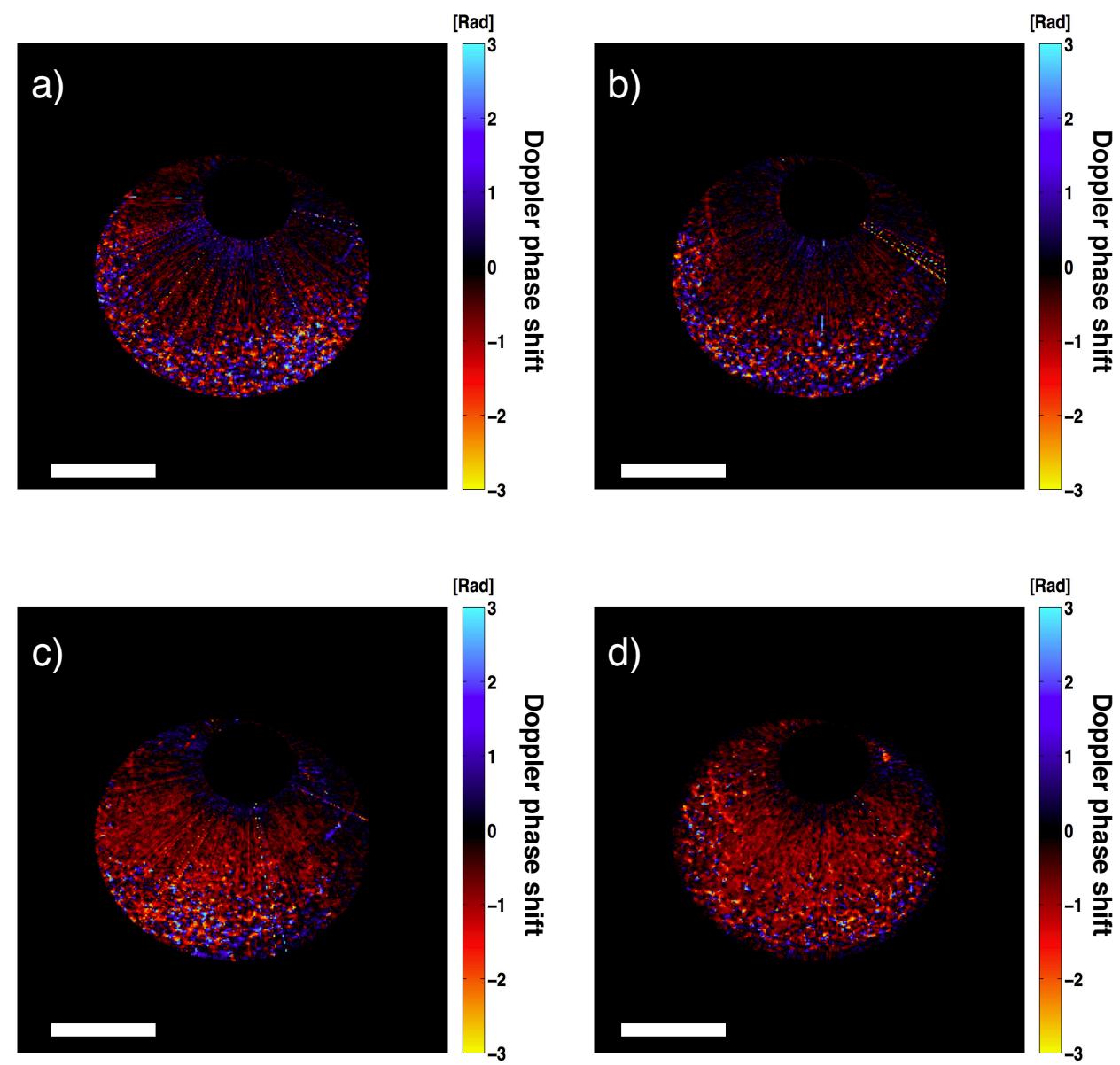

Figure 2.5: Imaging of a slow flow phantom at various blood and saline concentration. (a) DOCT image of $10 \%$ blood in saline mixture within the tube. (b) DOCT image of a blood in saline mixture of $5 \%$. (c) DOCT images were then taken after the blood in saline mixture was reduced to $2.5 \%$. (d) Finally, DOCT image acquired with a blood in saline mixture of $1.5 \%$. Scale bars $=1 \mathrm{~mm}$.

determine the degree of carotid stenosis and establish baseline intracranial circulation in comparison to extracranial circulation, this catheter was placed in the external carotid artery. A larger catheter $(\sim 6 \mathrm{~F})$ was exchanged and situated in the common carotid artery. A distal embolic protection device (e.g., AngioGuard, Cordis Corp., Miami, USA) with its guidewire was then placed into the carotid artery. The guidewire is compatible with the intravascular OCT catheter using a monorail rapid exchange technique. Once the intravascular OCT catheter was positioned in the region of interest, a volumetric scan was performed to deduce the Doppler angle (Figure 2.6(a)). A cross-sectional DOCT image of both 2500 A-lines per frame and 500 A-lines per frame was acquired. It was observed that the 500 A-lines per frame was sufficient to resolve flow within the carotid artery (Figure 2.6(d)). The 2500 A-lines per frame provided ample sampling for velocity estimation. Uncompensated and compensated of DOCT images from a different trial are shown in Figure 2.6(e) and Figure 2.6(f), respectively. Doppler 

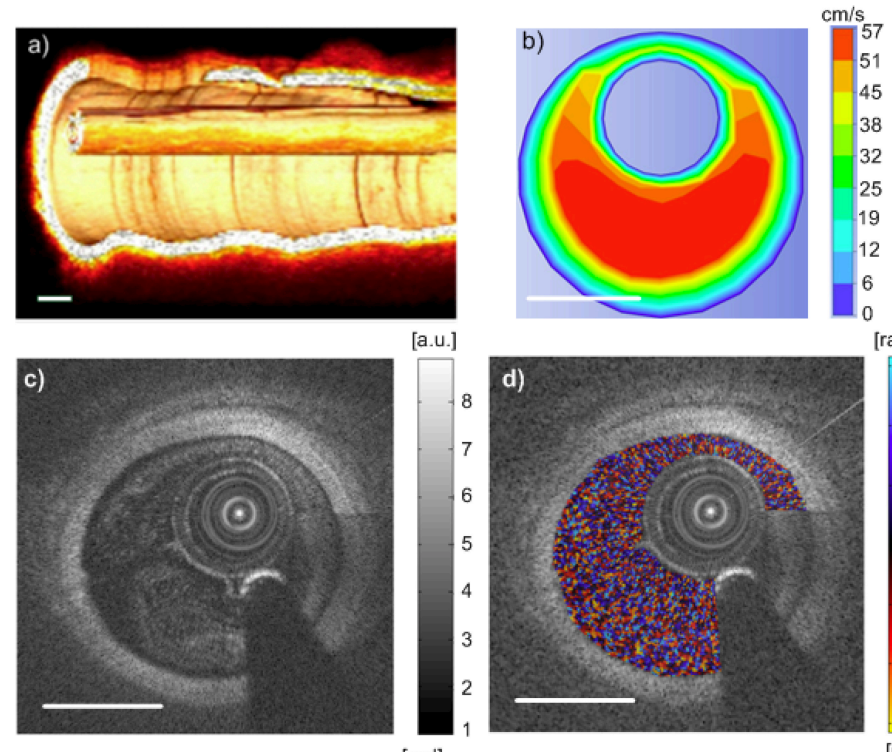

[rad]
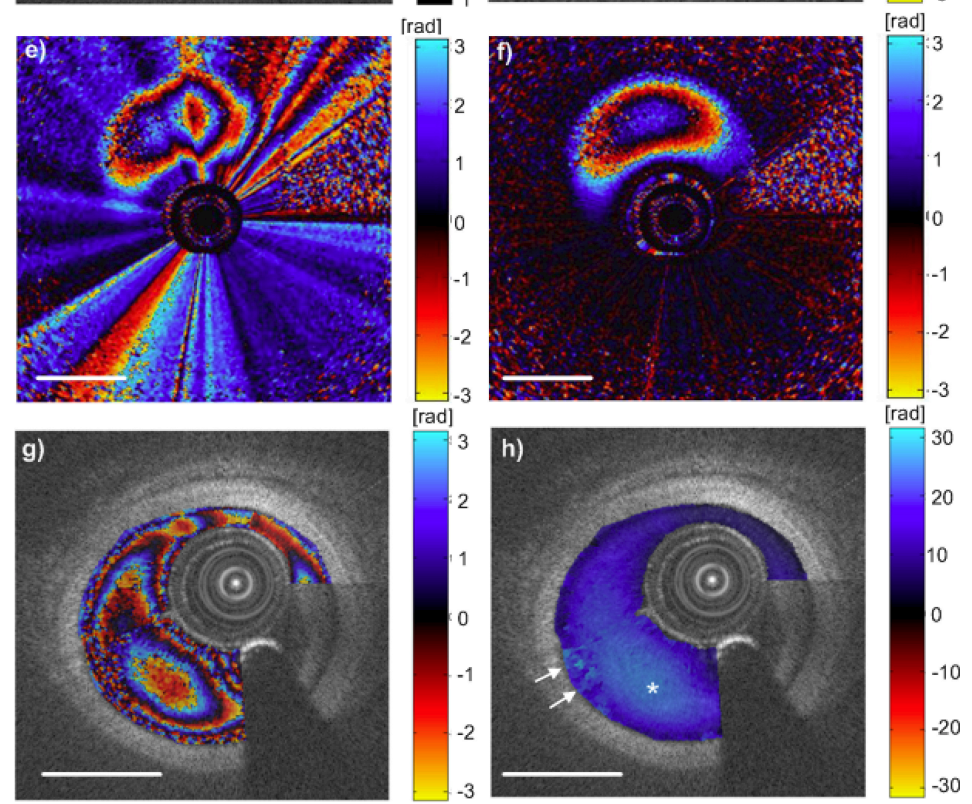

Figure 2.6: In vivo endovascular flow measurement. (a) 3D OCT image of the catheter and the vessel wall, which shows the angle between the catheter and the wall is $\sim 10^{\circ}$. (b) Simulation results of blood flow. (c) Cross-sectional OCT image of a porcine carotid artery with shadow casted by guide wire. (d) The same cross-section as (c) imaged by 500 A-lines/frame with Doppler shift overlaid, showing mainly noise. (e) and (f) (Media 1). (e) Phase shift image, 2500 A-lines/frame, without NURD induced phase artifact removal. It shows distorted phase contour lines. (f) Phase shift image after NURD induced phase artifact removal by tracking the phase shift in the catheter sheath. The corrected phase contour lines are as expected. The NURD induced artifact is time variant, as shown by Media 1. (g) A typical cross-sectional frame, 2500 A-lines/frame with Doppler shift overlaid, showing aliased phase changes. (h) The unwrapped phase map of (g), where * indicates the highest velocity region. The arrows indicate incorrect phase unwrapping due to noise and high shear rate near the vessel wall. Scale bars $=1 \mathrm{~mm}[3]$. (Reprinted with permission of the Optical Society of America) 
phase contours were susceptible to the NURD and Doppler artefacts induced by motion artefacts as significant distortions and asymmetry of the Doppler phase profile were observed. After compensation, the expected symmetric Doppler phase profile was correctly estimated. High velocities is present in the ICA which would result in the formation of aliasing phase contours (Figure 2.6(g)). This leads to difficulty in quantifying peak velocity. Previous studies have employed phase unwrapping techniques to calculate high flow velocities [3]. A quality-guided phase unwrapping algorithm [54] was utilized to unwrap the Doppler phase image in Figure 2.6(g). A mask was used in order to achieve a successful phase unwrapping of the alias rings. This is one of the limitation of the phase unwrapping method, which will be discuss later. The resultant image demonstrated a peak velocity of $\sim 24 \mathrm{rad}$, which responds $\sim 51$ $\mathrm{cm} / \mathrm{s}$ (Doppler angle of 80 degrees). It can be seen from this figure that the multiple phase contours correspond to high velocity. A computational fluid dynamics model (CFD, ANSYS, V8.0), was used to verify the unwrapped velocity measurement. Blood was assumed to be incompressible with a fluid density of $1060 \mathrm{~kg} / \mathrm{m}^{3}$ and a viscosity of $0.003 \mathrm{~Pa} \cdot \mathrm{s}$. The simulated vessel had a diameter of $2.5 \mathrm{~mm}$ and the catheter diameter was $0.9 \mathrm{~mm}$ as measured from the structural OCT image. This simulation resulted in a peak velocity of $\sim 57 \mathrm{~cm} / \mathrm{s}$. The expected and measured values are considered to be comparable, as the CFD model is idealized. Mainly, the assumptions in the simulation were a non-deformable straight tube with homogenous material properties, fluid density and viscosity of blood. Overall, this initial work demonstrated the feasibility of intravascular DOCT imaging. To the best of our knowledge, this work was the first demonstration of DOCT imaging in rotary catheter in the carotid artery.

\subsection{Split spectrum Doppler optical coherence tomography}

In the previous section, it was observed that the Doppler noise floor was $\sim 0.02 \mathrm{rad}$ (internal reflection), while the fundamental limit or phase stability of the system is $\sim 0.01 \mathrm{rad}$. Improved phase stability of the OCT laser source and its wavelength sweeping synchronization (swept source OCT) with the data acquisition, are critical in the design of a sensitive DOCT system. Improved phase stability and synchronization can be achieved in a variety of methods, including measuring the phase of a stationary reflective element or compensating for the unstable phase numerically [38,55]. Methods that do not require additional optical or electronic components have also been demonstrated [56].

It was seen in section 2.1 that the ability to resolve Doppler shifts are degraded by mechanical vibrations induced by the rotation of the optical components. Regions were carefully selected during histogram-based velocity segmentations to correct for these artefacts. Intra-frame techniques like the Kasai autocorrelation estimator have been reported to be less sensitive to bulk motion from tissue and probe [51]. An ideal method for improving degraded Doppler images involves averaging over multiple frames of the same location. In practice, this method may be difficult to achieve due to the inter-frame displacements, which are influenced by breathing [57], blood pulsation [58], or other environmental motion. 


\subsubsection{Split spectrum Doppler optical coherence tomography theory}

The concept of split spectrum was first derived in radar to resolve concealed targets in randomly distributed reflection, known as clutter [59]. This concept was later applied to ultrasound as a frequency agility technique for flaw detection in materials without the common reduction of spatial resolution associated with axial averaging. It has been suggested that the decomposition of the received wideband ultrasound signal into a narrowband ensemble results in higher SNR than conventional temporal averaging [60].

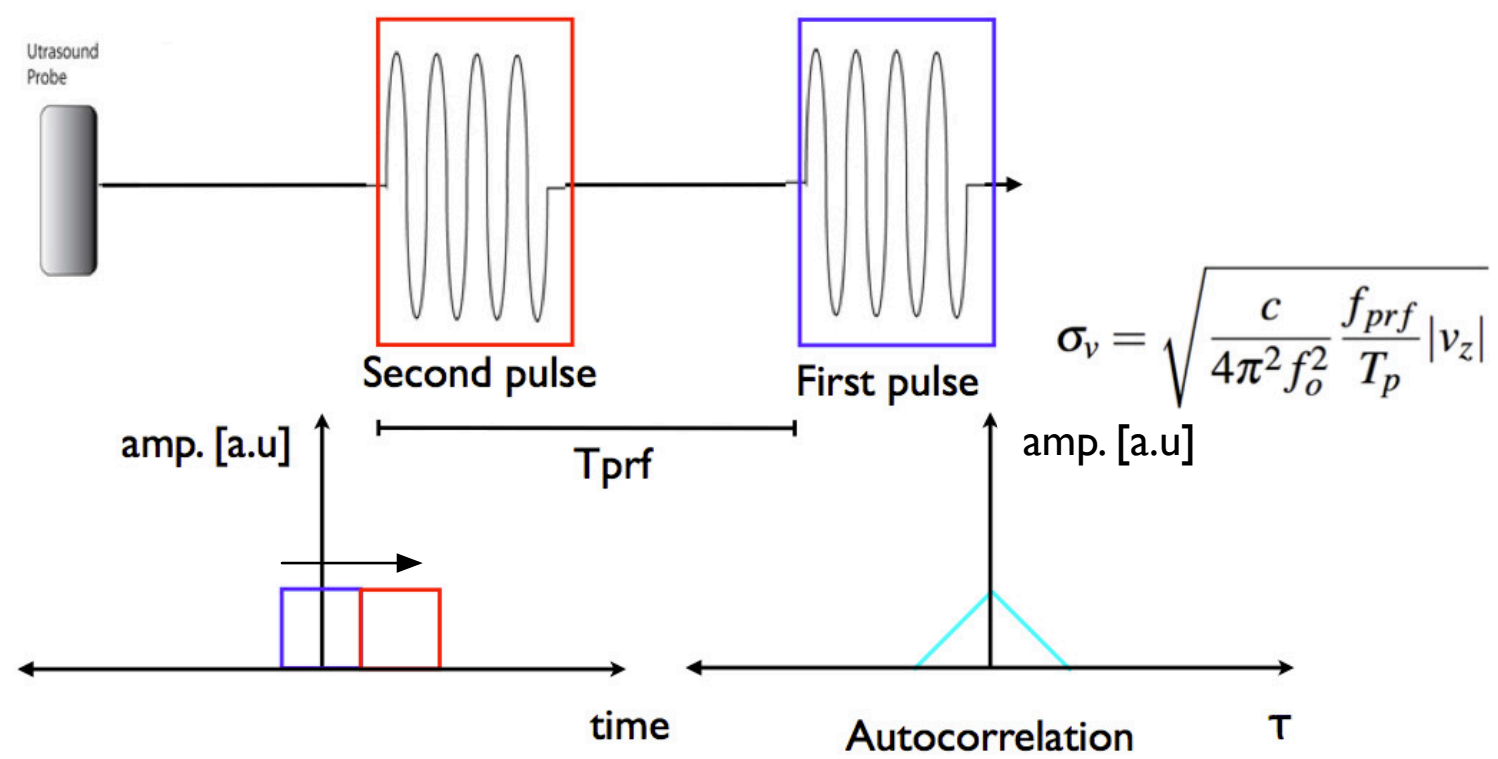

Figure 2.7: A simplified diagram that depicts two square wave (blue and red box) pulses emitting from an ultrasound transducer (top diagram). The result of the autocorrelation of these square waves results in a triangle waveform, which was used to derived the standard deviation of the Doppler shift using Eq. 2.1. It can be seen that a longer pulse length $\left(T_{p}\right)$ can result in a decrease in standard deviation given a transducer center frequency $\left(f_{o}\right)$ and the speed of sound (c). However, an increase in pulse repetition frequency $\left(f_{p r f}=\frac{1}{T_{p r f}}\right)$ and velocity of the moving scatterer $\left(v_{z}\right)$ would increase the standard deviation of the Doppler shift.

In the field of ultrasound, the transducer bandwidth has influence in the standard deviation of velocity measurements. It has been established that the narrowing of the frequency spectrum leads to a longer-range gate and higher velocity resolution. Generally, longer-range gate results in high amounts of power deposition leading to higher SNR [61]. Jensen [62] derived an inverse relationship between pulse duration (or transducer bandwidth) and standard deviation of Doppler shift based on a two square wave pulse system (Figure 2.7), though this does not apply to OCT. However, the foundation of the variance in Doppler shift can be applied to OCT and is demonstrated by the following [62]:

$$
\sigma_{D}^{2} \approx \frac{2}{T_{p r f}^{2}}\left(1-\frac{R_{g g}\left(T_{p r f}\right)}{R_{g g}(0)}\right)=\frac{2}{T_{p r f}^{2}}\left(1-\frac{\left|R\left(T_{p r f}\right)\right|}{R(0)}\right)
$$


where $T_{p r f}$ is the pulse repetition period, $\left|R\left(T_{p r f}\right)\right|$, is determined by the autocorrelation function of the envelope of the pulse, $R(0)$ is the power of the received signal, and $R_{g g}\left(T_{p r f}\right)$ denotes the value found from correlating two measured RF lines. The derivation of this equation can be found in Appendix 2 .

The time displacement between two RF lines is

$$
t_{s}=\frac{2 v_{z}}{c} T_{p r f}
$$

where $\mathrm{c}$ is the speed of sound and $v_{z}$ is the axial velocity of the sample.

In OCT, the axial displacement between two adjacent A-lines is

$$
\Delta z=v_{z} T=\frac{v_{z}}{f_{a}},
$$

where $f_{a}$ is the axial scan rate of the OCT. When the velocity increases, the Doppler shift estimation error increases [2] as $\Delta z$ becomes increasingly larger in comparison to the coherence gate $\left(l_{c}\right)$ of a time-domain OCT system.

In the past decade, the OCT research field has advanced from time-domain to frequency domain OCT, including swept source OCT. Yun et al. [63] (Eq. (18)) modelled the depth profile of a swept source OCT in terms of depth $(\mathrm{Z})$, within a coherence volume $(C V(x, y, z))$

$$
F(Z) \approx C V(x, y, z) \int_{-\infty}^{\infty} \exp \left(-4 \ln 2 \frac{k^{2}}{\left(2 k_{1} T \sigma\right)^{2}}\right) \exp \left(i\left(2 k_{o}+k\right)\left(Z-z_{o}-\frac{v_{z}}{2 k_{1}} k\right)\right) d k
$$

where

$$
C V(x, y, z)=\gamma P_{o} \iiint r(x, y, z) g\left(x-x_{b}, y-y_{b}\right) \exp \left(-i 2 k_{o} Z\right) d x d y d z,
$$

The relationship between the axial profile and the swept source OCT system characteristics can be shown as:

$$
\begin{aligned}
& F(Z) \propto \gamma P_{o} \iiint r(x, y, z) \exp \left(-i 2 k_{o} z_{o}\right) \exp \left(-4 \ln 2 \frac{\left(x-x_{b}\right)^{2}}{w_{o}^{2}}\right) \\
& \quad \exp \left(-4 \ln 2 \frac{\left(y-y_{b}\right)^{2}}{w_{o}^{2}}\right) \exp \left(-4 \ln 2 \frac{\left(Z-\left[z_{o}+\left(k_{o} / k_{1} T\right) \Delta z\right]\right)^{2}}{\delta z_{o}^{2}\left(1+4 \sigma^{2} \Delta z^{2} / \delta z_{o}^{2}\right)}\right) d x d y d z,
\end{aligned}
$$

where $\gamma$ is the photon-to-electron conversion efficiency, $P_{o}=\sqrt{P_{r}(t) P_{s}(t)}, P_{r}(t)$ is the optical power returned from the reference arm, $P_{s}(t)$ is the optical power turned from the sample arm (sample at $100 \%$ reflection), $r(x, y, z)$ denote the complex-valued backscattering coefficient, $g(x, y, z)$ represents the intensity profile of the probe beam in the sample arm, $\left(x_{b}, y_{b}, z_{b}\right)$ is the coordinates of the probe beam at zero path length difference of the interferometer, $w_{o}$ is the beam waist, $\sigma k_{1} T$ is the full width half maximum (FWHM) tuning range in wavenumber, $k(t)=k_{o}+k_{1} t$ is the output wavenumber that is tuned linearly in time, and $\delta z_{o}=\frac{4 \ln (2)}{k_{1} T \sigma} \mathrm{FWHM}$ axial resolution, equivalent to $l_{c}$, the coherence gate in a time domain system. The FWHM tuning envelop of the swept source laser is defined by $\sigma T$, where $T$ is the tuning period (A line rate). Typical swept source laser have a $\sigma$ between 0.5 to 0.8 [63].

From Equation 2.6, we let $\Upsilon$ be the coherent sum of all backscattered light from a coherence region 
that has a size of $w_{0}$ by $w_{0}$

$$
\Upsilon=\gamma P_{o} \iiint r(x, y, z) \exp \left(-i 2 k_{o} z_{o}\right) \exp \left(-4 \ln 2 \frac{\left(x-x_{b}\right)^{2}}{w_{o}^{2}}\right) \exp \left(-4 \ln 2 \frac{\left(y-y_{b}\right)^{2}}{w_{o}^{2}}\right) d x d y d z
$$

Then Equation 2.6 is simplified to,

$$
F(Z) \propto \Upsilon \exp \left(-4 \ln 2 \frac{\left(Z-\left[z_{o}+\left(k_{o} / k_{1} T\right) \Delta z\right]\right)^{2}}{\delta z_{o}^{2}\left(1+4 \sigma^{2} \Delta z^{2} / \delta z_{o}^{2}\right)}\right),
$$

To simplify the equation, let

$$
z^{\prime}=z_{o}+z_{D}
$$

and $[63]$

$$
z_{D}=\frac{k_{o}}{k_{1} T} \Delta z=\frac{\pi \sigma}{2 \ln 2} \frac{\delta z_{o}}{\lambda} \Delta z
$$

Therefore Equation 2.8 becomes

$$
F(Z) \propto \Upsilon \exp \left(-4 \ln 2 \frac{\left(Z-z^{\prime}\right)^{2}}{\delta z_{o}^{2}\left(1+4 \sigma^{2} \Delta z^{2} / \delta z_{o}^{2}\right)}\right) .
$$

When measuring the Doppler shift between two adjacent A-line scans, the autocorrelation of the depth profiles is calculated. From the Wiener-Khinchin theorem, the autocorrelation function is the following:

$$
\overline{F(Z)} \cdot F(Z)=R_{g g}(\tau)=\int_{-\infty}^{\infty} f(\tau) f(\tau+t) d \tau,
$$

where $F(Z)$ is the Fourier transform of $f(k)$ and $\overline{F(Z)}$ is the complex conjugate

If the initial A-line scan is $F_{T_{o}}(Z)$ and the adjacent A-line scan is $F_{T_{1}}$ then,

$$
F_{T_{o}}(Z) \propto \Upsilon \exp \left(-4 \ln 2 \frac{\left(Z-z_{T_{o}}^{\prime}\right)^{2}}{\delta z_{o}^{2}\left(1+4 \sigma^{2} \Delta z^{2} / \delta z_{o}^{2}\right)}\right)
$$

and

$$
F_{T_{1}}(Z) \propto \Upsilon \exp \left(-4 \ln 2 \frac{\left(Z-z_{T_{1}}^{\prime}\right)^{2}}{\delta z_{o}^{2}\left(1+4 \sigma^{2} \Delta z^{2} / \delta z_{o}^{2}\right)}\right) .
$$

The autocorrelation between the two adjacent lines is

$$
\overline{F_{T_{1}}(Z)} \cdot F_{T_{o}}(Z)=\bar{\Upsilon} \exp \left(4 \ln 2 \frac{\left(Z-z_{T_{1}}^{\prime}\right)^{2}}{\delta z_{o}^{2}\left(1+4 \sigma^{2} \Delta z^{2} / \delta z_{o}^{2}\right)}\right) \cdot \Upsilon \exp \left(-4 \ln 2 \frac{\left(Z-z_{T_{o}}^{\prime}\right)^{2}}{\delta z_{o}^{2}\left(1+4 \sigma^{2} \Delta z^{2} / \delta z_{o}^{2}\right)}\right)
$$




$$
=|\Upsilon|^{2} \exp \left(-4 \ln 2 \frac{2 Z \Delta z^{\prime}}{\delta z_{o}^{2}\left(1+4 \sigma^{2} \Delta z^{2} / \delta z_{o}^{2}\right)}\right) \exp \left(4 \ln 2 \frac{z_{T_{1}}^{\prime 2}-z_{T_{o}}^{\prime 2}}{\delta z_{o}^{2}\left(1+4 \sigma^{2} \Delta z^{2} / \delta z_{o}^{2}\right)}\right),
$$

where $\Delta z^{\prime}$ is difference in the depth of a moving scatterer and is related to its velocity. This equation determines OCT equivalent to $R_{g g}(\tau)$ in Equation 2.1. $R_{g g}\left(T_{p r f}\right)$ is the value evaluated from correlating the RF lines in ultrasound. In OCT the value from the correlating the two depth measurements is $R_{g g}\left(\Delta z^{\prime}\right)$ and $\Delta z^{\prime}=z_{T_{1}}^{\prime}-z_{T_{0}}^{\prime}=v_{z} T$. As a result we substituted $Z=\Delta z^{\prime}$, Equation 2.16 becomes

$$
R_{g g}\left(\Delta z^{\prime}\right)=|\Upsilon|^{2} \exp \left(-4 \ln 2 \frac{2 \Delta z^{\prime 2}}{\delta z_{o}^{2}\left(1+4 \sigma^{2} \Delta z^{2} / \delta z_{o}^{2}\right)}\right) \exp \left(4 \ln 2 \frac{z_{T_{1}}^{\prime 2}-z_{T_{o}}^{\prime 2}}{\delta z_{o}^{2}\left(1+4 \sigma^{2} \Delta z^{2} / \delta z_{o}^{2}\right)}\right)
$$

The power of the received signal, $R(0)$ is

$$
R_{g g}(0)=|\Upsilon|^{2} \exp \left(4 \ln 2 \frac{z_{T_{1}}^{\prime 2}-z_{T_{o}}^{\prime 2}}{\delta z_{o}^{2}\left(1+4 \sigma^{2} \Delta z^{2} / \delta z_{o}^{2}\right)}\right) .
$$

Inserting Equation 2.17 and Equation 2.18 into Equation 2.1 yields

$$
\begin{gathered}
\sigma_{D}^{2} \approx 2 f_{a}^{2}\left(1-\frac{\left|R\left(\Delta z^{\prime}\right)\right|}{R(0)}\right) \\
\approx 2 f_{a}^{2}\left(1-\exp \left(-4 \ln 2 \frac{2 \Delta z^{\prime 2}}{\delta z_{o}^{2}\left(1+4 \sigma^{2} \Delta z^{2} / \delta z_{o}^{2}\right)}\right)\right) \\
\approx 2 f_{a}^{2}\left(1-\exp \left(-4 \ln 2 \frac{2\left(v_{z} T\right)^{2}}{\delta z_{o}^{2}\left(1+4 \sigma^{2}\left(v_{z} T\right)^{2} / \delta z_{o}^{2}\right)}\right)\right) .
\end{gathered}
$$

The standard deviation of the Doppler shift estimate is then [4]

$$
\sigma_{D} \approx f_{a} \sqrt{2\left(1-\exp \left(-4 \ln 2 \frac{2\left(v_{z} T\right)^{2}}{\delta z_{o}^{2}\left(1+4 \sigma^{2}\left(v_{z} T\right)^{2} / \delta z_{o}^{2}\right)}\right)\right)} .
$$

The equation is applicable to both time domain and swept source OCT systems. In Chapter 1, it was shown that there is an increase in standard deviation of phase as a function of set velocities based on work by Yang et al. [2]. Yang et al. [2] had estimated an inverted Gaussian curve without any developed theoretical basis. The standard deviation of the Doppler shift estimate derived above shows the relationship with velocity, as well as other factors. Figure 2.8 shows the result of the equation given the characteristics of the original TD-OCT system $\left(l_{c}=12 \mu \mathrm{m}\right)$. It can be seen that Equation 2.22 demonstrated good agreement with the previously reported data. We also showed that there is a decrease in standard deviation of velocity estimate when the coherence length is lengthened (narrowing in the spectrum) by a fraction of the available bandwidth (FBW).

Recently, the SSP technique has been utilized in OCT to increase amplitude-decorrelation SNR and reduce speckle. In both of these applications, the SSP was only applied to the magnitude intensity of the OCT signal and not to phase measurements. In an effort to overcome the limitations of conventional 


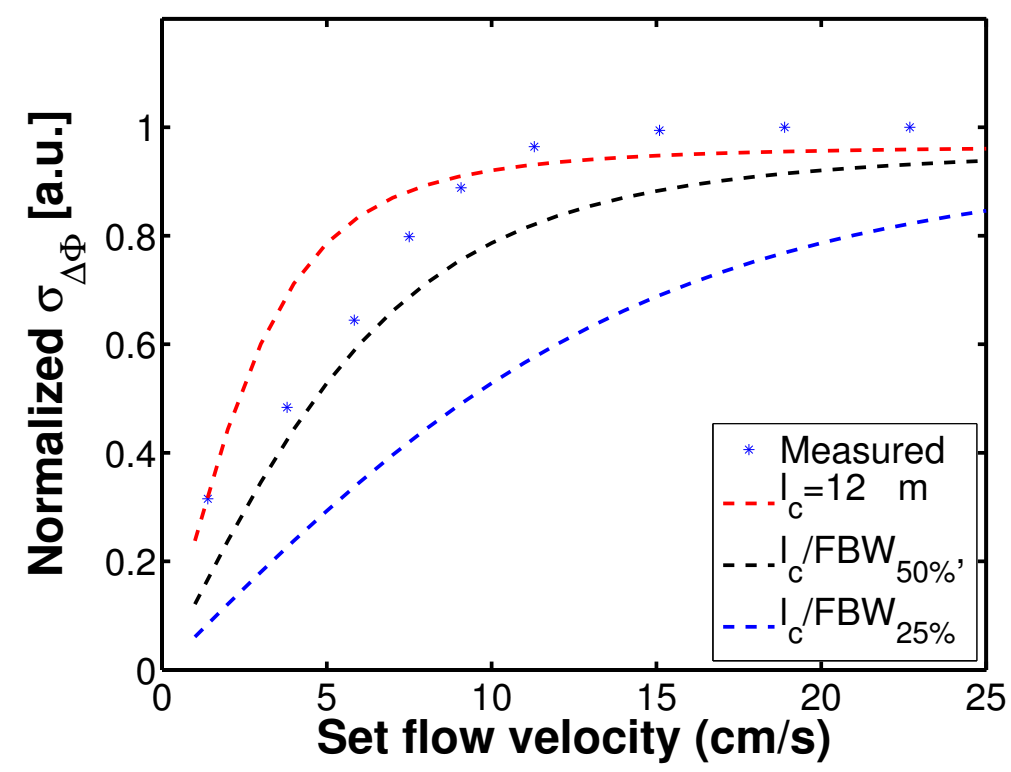

Figure 2.8: Normalized standard deviation of phase shift $\left(\sigma_{\Delta \Phi}\right)$ versus flow velocity. The result of Equation 2.22 with the coherence length $\left(l_{c}\right)$ of the system from [2] was plotted. Coherence length was increased by a factor of 2 and 4 (spectral bandwidth decreases to $50 \%$ and $25 \%$ ) via fractional bandwidth (FBW) and the respective phase noise $\left(\sigma_{\Delta \Phi}\right)$ of were plotted as well [4]. (Reprinted with permission of the Optical Society of America)

averaging techniques, a novel intra-frame DOCT clutter-filtering algorithm was developed, which we term split spectrum Doppler OCT (ssDOCT). The algorithm for split spectrum velocity estimation, in its most basic form, requires calculating the Doppler shift from the interferogram that is split into multiple narrow window bands. Figure 2.9 shows this basic form of the ssDOCT algorithm, where window functions $W_{b}\left(k, k_{o}\right)$ splits the interferogram $S(k)$ into multiple bands. To ensure no clipping at the border of the available range, the bands have a continuous profile and are overlapped such that it spans the entire spectrum of the interferogram. This reduces any artefacts and/or loss of information in the DOCT signal. Each of these bands is also equally spaced across the entire interferogram, with a center wavenumber of $k_{o}$. The inverse fast Fourier transformed $\left(F^{-1}\right)$ evaluated each zero-padded (to the length of the original record length) band, resulting in a complex OCT signal $(S)$ for each band $(b)$ with respect to the optical path difference $(z)$ between the sample and reference arms.

As a result, the Kasai velocity estimator in Chapter 1 (Equation 1.9) was modified to incorporate the split bands resulting in the following. 


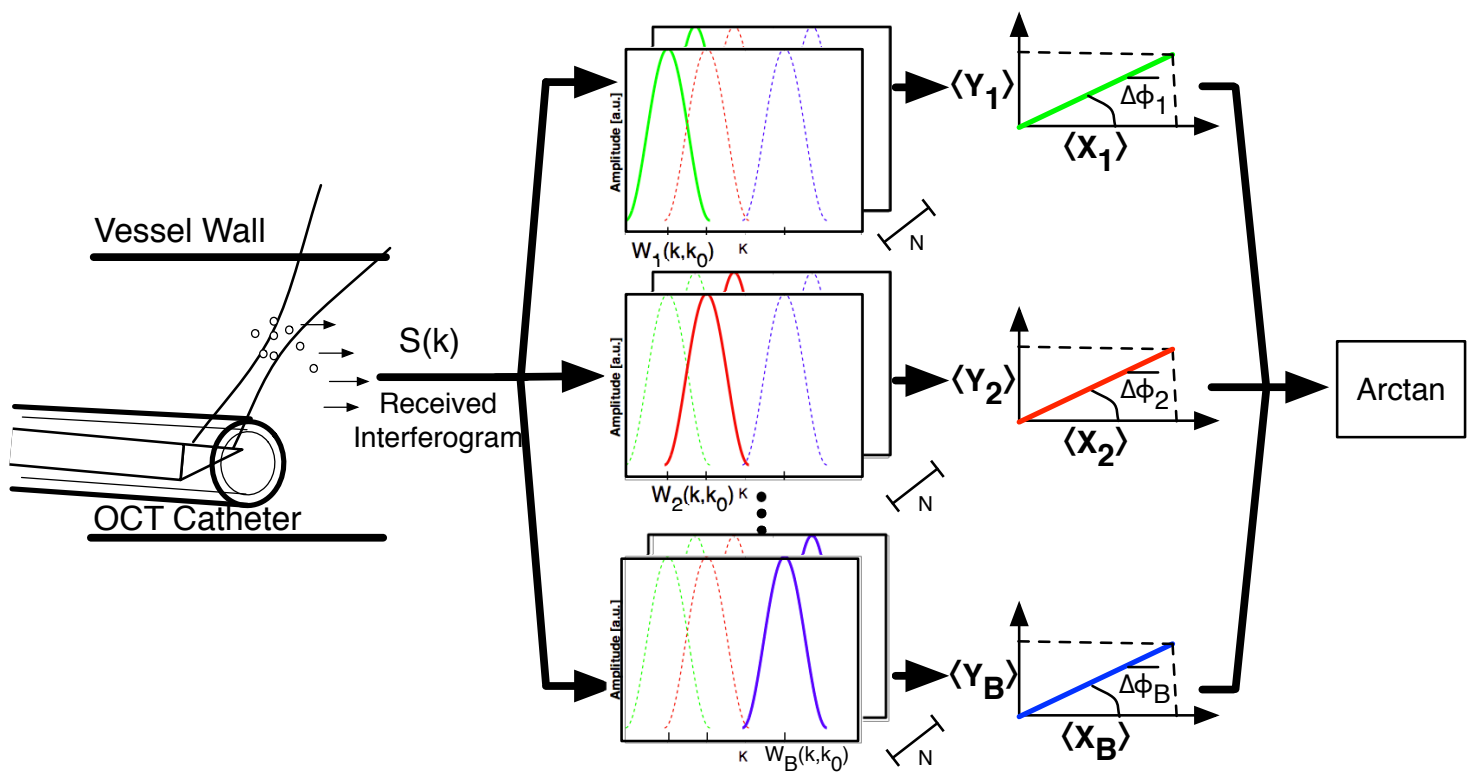

Figure 2.9: Data acquisition and processing scheme. The OCT rotary catheter was inserted into a vessel and images were acquired during blood flow. Multiple narrow window bands with different center frequencies were multiplied with the interferogram. These windowed spectra were stored into the corresponding windowed band frame and the mean phase shift was calculated by evaluating the phase difference between axial scans within the frame (Equation 2.23) [4]. (Reprinted with permission of the Optical Society of America) 


$$
\begin{gathered}
f_{D}=\frac{f_{a}}{2 \pi} \arctan \left\{\frac{\frac{1}{B M(N-1)} \sum_{b=1}^{B} \sum_{m=1}^{M} \sum_{n=1}^{N-1}\left(I_{b, m, n+1} Q_{b, m, n}-Q_{b, m, n+1} I_{b, m, n}\right)}{\frac{1}{B M(N-1)} \sum_{b=1}^{B} \sum_{m=1}^{M} \sum_{n=1}^{N-1}\left(Q_{b, m, n+1} Q_{b, m, n}+I_{b, m, n+1} I_{b, m, n}\right)}\right\} \\
=\frac{f_{a}}{2 \pi} \arctan \left\{\frac{\frac{1}{B} \sum_{b=1}^{B}\left\langle Y_{b}\right\rangle}{\frac{1}{B} \sum_{b=1}^{B}\left\langle X_{b}\right\rangle}\right\} .
\end{gathered}
$$

The formation of a 2D structural OCT was also modified to the following

$$
\left\langle S^{2}\right\rangle=\frac{1}{B M N} \sum_{b=1}^{B} \sum_{m=1}^{M} \sum_{n=1}^{N}\left[I_{b, m, n}^{2}+Q_{b, m, n}^{2}\right]
$$

\subsubsection{Doppler noise floor analysis}

From Equation 2.23, there are several parameters such as bandwidth of each band, the amount of overlap between the window bands, and the number of window bands that could influence Doppler phase noise. Optimization of these parameters was investigated using a Hamming apodization window $W_{b}\left(k, k_{o}\right)$ and the same characterization methods as the previous section were employed. The amount of overlap between bands is characterized by FBW and is denoted as $F B W=F W H M_{b} / F W H M_{\text {full }} \%$, where it is the ratio between the full width half maximum (FWHM) of a single band $\left(F W H M_{b}\right)$ and the FWHM of the full window $F W H M_{\text {full }}$.

A high SNR $(\sim 6 \mathrm{~dB})$ and a low SNR $(\sim 2 \mathrm{~dB})$ region of the stationary phantom were selected. The mean standard deviation of phase $(\Delta \Phi)$ with a Kasai autocorrelation window ensemble length $\mathrm{N}=10$ was evaluated over 20 frames and shown in Figure 2.10(a) and Figure 2.10(b). This figure depicts various percentages of FBW and number of window bands (B) is investigated, as well as compared to the conventional Kasai window averaging in the axial direction (M). It was observed that the utilization of multiple bands suppressed Doppler phase noise. There were also a local minima in the background Doppler phase noise as a result of ssDOCT in both high SNR and low SNR regions. As the percentage of FBW increased, the overlaps of bands approach the single apodization window condition $(B=1)$, which resulted in the increase of standard deviation of phase $(\Delta \Phi)$. In this single apodization window condition, the non-split interferogram utilized the maximum resolution and spectral power. In the ssDOCT algorithm, the interferogram was split into narrow bands, which Equation 2.22 demonstrates is a decreased standard deviation of the velocity estimation. However, one of the consequences of the narrow bands is the decreased spectral power for flow velocity estimation. The averaging over each of the unique bands results in higher velocity resolution while most of the spectral power is recovered.

As the percentage of FBW decreased, the bands become sparsely spaced leading to limited or no overlap, producing uncorrelated signals and potential artefacts. When this occurs there would be a loss of information between the filter bands. Thus, the selection of the FBW parameter was necessary in order to minimize the Doppler phase noise. This was particularly critical in low SNR regions as the local minima became more pronounced (Figure 2.10). When the FBW and B parameters were carefully chosen, a larger suppression of the Doppler phase noise was observed in low SNR regions compared to 

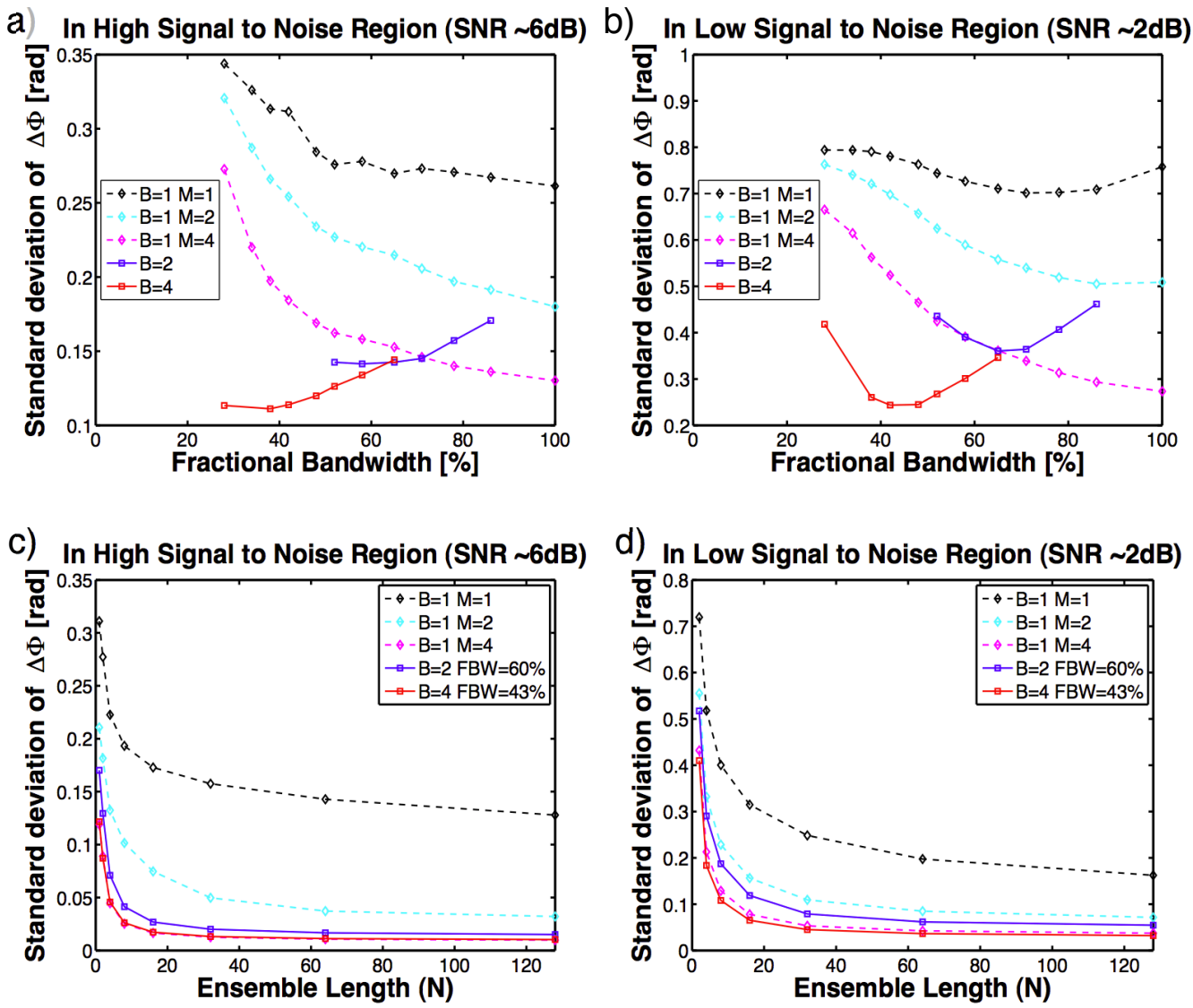

Figure 2.10: A series of 20 structural OCT images were taken in B-mode of a stationary tissue phantom. (a) and (b): mean measurements of background phase noise (standard deviation of $\Delta \Phi$ ) using DOCT and ssDOCT at different fractional bandwidth (FBW) for high SNR(approx. 6dB) region and low SNR (approx. 2dB) region at an ensemble length $\mathrm{N}=10$. In all ssDOCT calculations, $\mathrm{M}=1$. The catheter beam was then set at a static position and 20 structural OCT images were taken in M-mode. (c) and (d): mean measurements of back- ground phase noise using DOCT and ssDOCT at various ensemble lengths (N) for high SNR region and low SNR region [4]. (Reprinted with permission of the Optical Society of America) 
their equivalent axial averaging.

In order to further understand the effects of ssDOCT, the catheter was held in a static position. Large ensemble averaging in the transverse direction $(\mathrm{N})$ would be difficult to achieve as the beam overlap between each A-line scan was finite. Meanwhile, insufficient beam overlap can result in poor velocity estimates. The static position of the catheter permitted large ensemble lengths to be evaluated and compared between the conventional DOCT and ssDOCT (Figure 2.10(c) and Figure 2.10(d)). It was observed that utilizing $\mathrm{B}=2$ and $\mathrm{B}=4$ in ssDOCT resulted in a Doppler phase noise convergence at $\mathrm{N}=32$. Similar performance had occurred when $\mathrm{M}=4$ for conventional DOCT algorithm. The performance of both DOCT and ssDOCT algorithm approached the limit of system performance as previously measured by the common path interferometer benchmark $\left(\Delta \Phi_{o}=0.01 \mathrm{rad}\right)$. Based on these findings of our Doppler phase noise characterization, ssDOCT provided comparable phase noise when averaging over 2 bands, while the same phase noise suppression can be achieved by averaging over 4 pixels in the axial direction. This had lead to a decrease in degradation of resolution when employing averaging over 2 bands rather than 4 pixels (conventional DOCT).

\subsubsection{Low velocity flow phantom analysis}

Comparison of conventional DOCT (left column) and ssDOCT (right column) was again evaluated through flow phantom models (Figure 2.11). The conventional structural OCT image was evaluated by Equation 1.9 (Figure 2.11(a)) and the SSP structural OCT image was evaluated by Equation 2.24 (Figure 2.11(b)). The corresponding Doppler shift images (DOCT $[\mathrm{B}=1, \mathrm{M}=2, \mathrm{~N}=10]$ Figure 2.11(c) $\& \operatorname{ssDOCT}[\mathrm{B}=4, \mathrm{M}=1, \mathrm{~N}=10]$ Figure 2.11(d)) depict the same artefacts as shown in the previous section. Histogram-based velocity segmentation compensation of conventional DOCT and ssDOCT are presented in Figure 2.11(e) and Figure 2.11(f), respectively. It was observed that less phase noise had influenced the Doppler image in ssDOCT than in DOCT at low SNR regions (1.7 $\mathrm{mm}$ to $2 \mathrm{~mm}$ from the catheter). To show the overall phase noise within both Doppler images, the DOCT and ssDOCT velocity profiles for the vessel were generated using an averaging filter with a $32 \times 32$ kernel. The result from the subtraction of the mean velocity profile with the respective compensated Doppler images can be seen in Figure 2.11(g) and Figure 2.11(h). The evaluation of the room mean square value of the background Doppler noise resulted in a lower RMS value in $\operatorname{ssDOCT}(\mathrm{RMS}=0.994 \mathrm{rad})$ than in DOCT $(\mathrm{RMS}=1.428$ $\mathrm{rad})$. A T-test had shown that this was statistically significant $(\mathrm{P}<0.01)$.

The improved Doppler noise suppression in ssDOCT can further be observed by comparing the flow profile with CFD simulations. Based on the dimensions of the flow phantom, an ANSYS simulation fitted phase shift model was constructed and shown in Figure 2.12(a). A single axial section (white dashed line) from the center of the catheter to the lumen wall was extracted from the CFD simulations. A narrow region of the same location in DOCT and ssDOCT phase measurements was also extracted and overlaid onto the CFD A-line (Figure 2.12(b)). The standard deviation of ssDOCT was observed to have nearly half the value on the whole $3 \mathrm{~mm}$ axial scan than DOCT. The various phase shifts in the entire flow profile of the ANSYS simulation fitted model (expected phase shift) was then plotted against the measured phase shift for both DOCT (Figure 2.12c) and ssDOCT (Figure 2.12(d)). Again, 


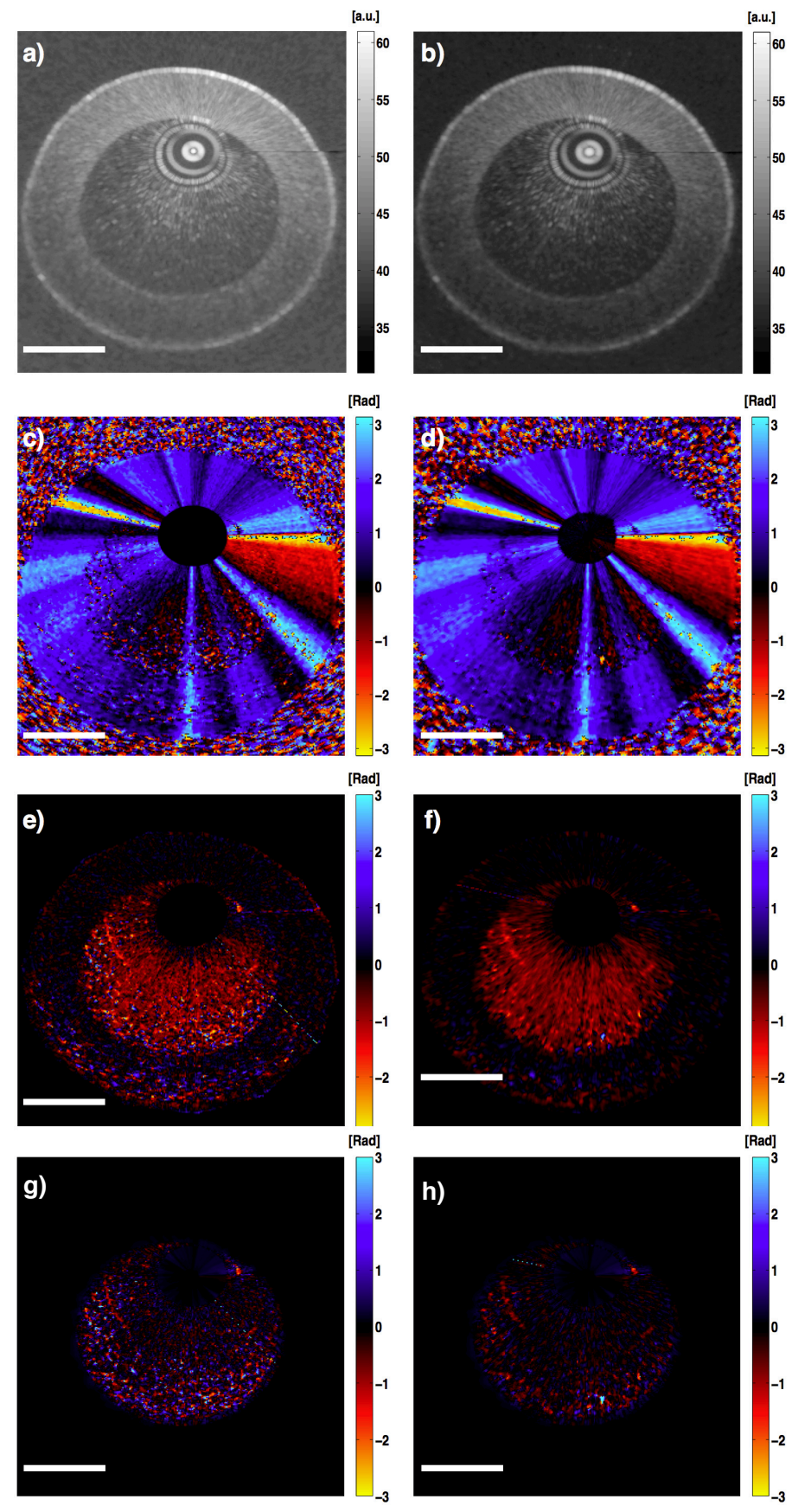

Figure 2.11: Structural image of a slow flow phantom with 1.5\% diluted blood mixture realized using the full length apodization window $(\mathrm{B}=1, \mathrm{M}=2, \mathrm{~N}=10)$. (b) The corresponding structural SSP-OCT image $(B=4, M=1, N=10)$. (c) and (d) The Doppler shift of the slow flow phantom evaluated by DOCT and ssDOCT, respectively. Doppler artefacts were present in both images. (e) and (f) The corrected Doppler image computed from DOCT and ssDOCT techniques, respectively. The relative phase from (g) DOCT and (h) ssDOCT was then visualized by subtracting the averaged velocity profile. More phase noise is present in DOCT compared to ssDOCT. Scale bar $=1 \mathrm{~mm}$ [4]. (Reprinted with permission of the Optical Society of America) 
it was observed that the standard deviation as a result of ssDOCT proccessing was reduced to nearly half while a significant reduction in outlier data points were seen. Tighter clustering of data points at lower velocity estimates $(<-0.1 \mathrm{rad})$ in ssDOCT compared to DOCT suggests that ssDOCT has the ability to detect lower velocities.

a)
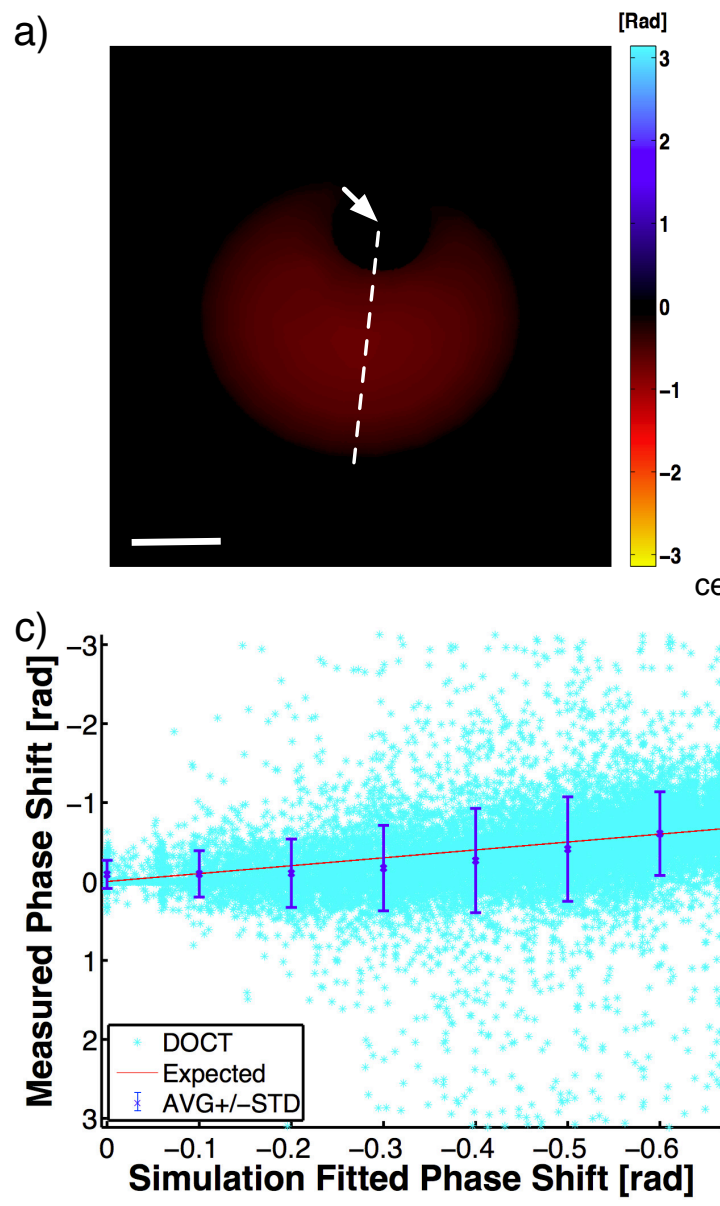

b)
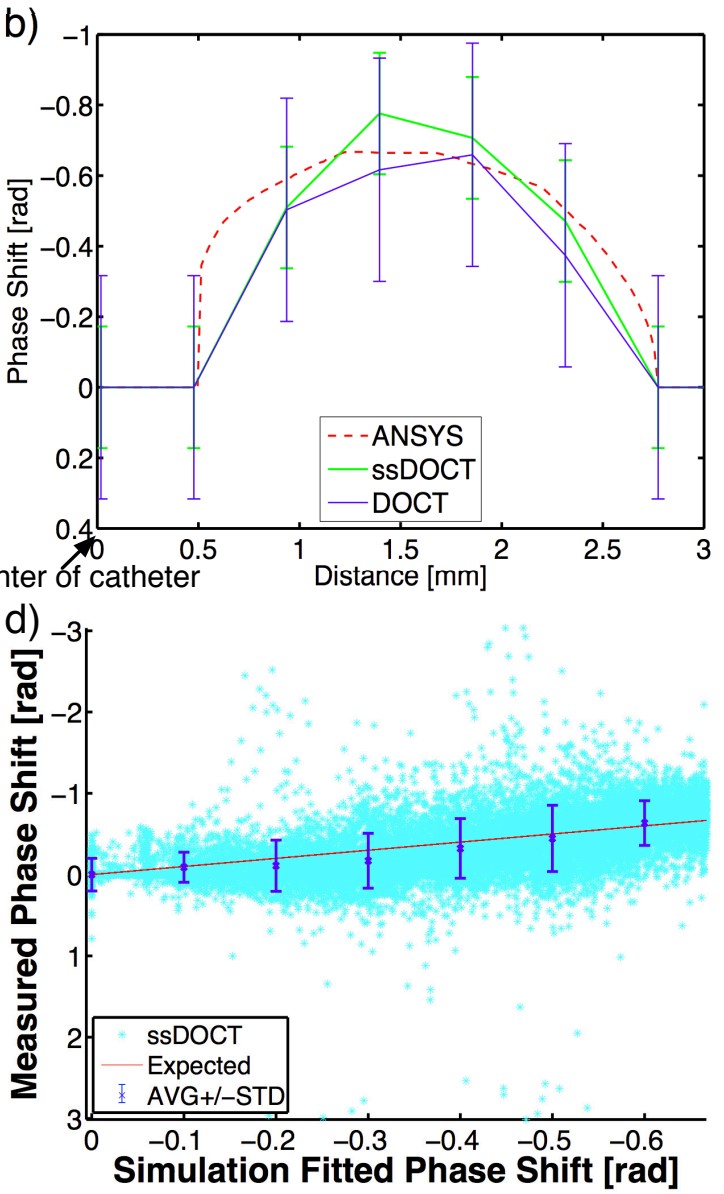

Figure 2.12: (a) An ANSYS simulation fitted phase shift model of Figure 2.11 was generated. Scale $=1 \mathrm{~mm}$ (b) The axial profile was taken (dashed line in (a)) from the center of the catheter (arrow) and the mean \& standard deviation were compared. The expected velocity profile was then plotted against the measured phase shift of (c) DOCT $(B=1, M=2, F B W=100 \%)$ and (d) ssDOCT $(B=4, M=1$, FBW=44\%). The expected phase shift (red) and mean \& standard deviation (blue) were plotted [4]. (Reprinted with permission of the Optical Society of America)

\subsubsection{In vivo split spectrum Doppler optical coherence tomography imaging}

High flow imaging in the carotid artery was initially evaluated using ssDOCT in the in vivo porcine model (Figure 2.13). As previously mentioned, phase aliasing between $-\pi$ to $\pi$ occurs during high flow velocities. In the case of the carotid artery, this produced phase contours in the form of rings. 
A comparative assessment of these aliasing phase rings suggested that there was a clearer delineation (arrow) in ssDOCT than DOCT techniques. The distinctions between the aliasing rings were lost in portions of the DOCT suggesting insufficient axial resolution. This degradation between unique rings would also degrade the velocity estimate.
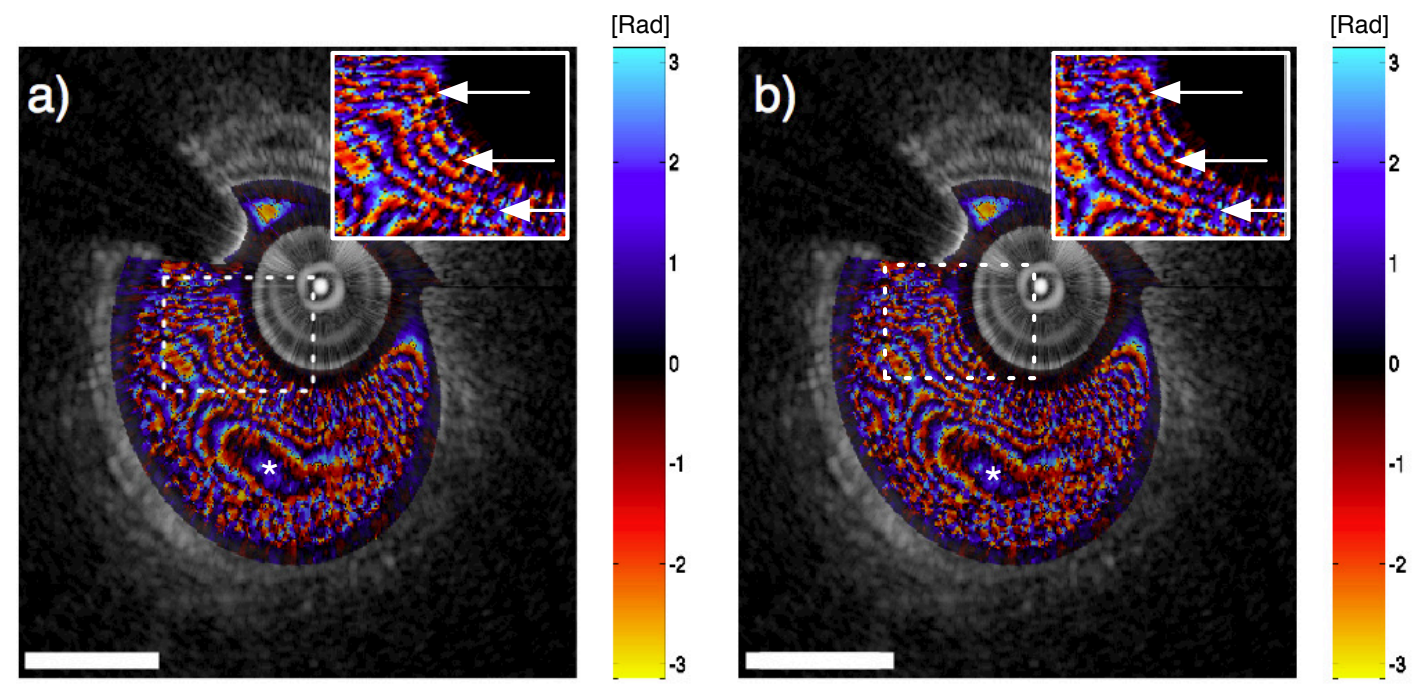

Figure 2.13: The structural OCT image overlapped with Doppler image of a high flow region in a porcine carotid artery. (a) Doppler images produced by DOCT ( $\mathrm{B}=1, \mathrm{M}=4, \mathrm{FBW}=100 \%$ ). (b) The corresponding $\operatorname{ssDOCT}(\mathrm{B}=2, \mathrm{M}=1, \mathrm{FBW}=60 \%)$ with approximately similar phase noise performance as shown in Figure 2.10(a). The arrows indicate less degradation in the visualization of the aliasing rings comparing (b) to (a). The unwrapped value in both DOCT and ssDOCT showed a peak velocity of $18.7 \pi$ (asterisk). Scale bar $=1 \mathrm{~mm}$ [4]. (Reprinted with permission of the Optical Society of America)

ssDOCT processing was also employed in low flow velocities in a stented carotid artery. As mentioned in Chapter 1, stents are commonly used for the treatment of aneurysms and vascular diseases. The interaction of blood and stents plays an important role in its design and application. To our knowledge, this was the first work to report direct visualization of in vivo flow patterns around stent struts using OCT with its inherent high spatial resolution and preserved by ssDOCT.

Conventional structural OCT and split spectrum OCT images of a deployed stent in the carotid artery can be seen in Figure 2.14(a) and Figure 2.14(b), as well as the corresponding compensated Doppler phase images of DOCT (Figure 2.14(c)) and ssDOCT (Figure 2.14(d)). Here the Doppler phase noise was indistinguishable from the vessel wall and stent struts. The same phase thresholded value was then applied to DOCT (Figure 2.14(e)) and ssDOCT (Figure 2.14(f)), which resulted in better visualization. For the case of ssDOCT, significant amount of Doppler phase noise occurred relative to DOCT. Prominent blood flow patterns can be seen in both techniques. For example a flow profile adjacent to one of the stent strut (lower *) was observed. This was believed to be an eddy current from that strut. A second perceived eddy current (upper *) was detected which may be caused from a stent strut upstream from the location of the OCT catheter. Finally ssDOCT resolved the weak Doppler 
signal exhibited from two stent struts (arrows) at the $8 \& 9$ O'clock direction and separated it from the background noise.

This chapter has outlined the development of an intravascular DOCT and ssDOCT. ssDOCT has a number of advantages over conventional DOCT; namely greater sensitivity in velocity estimation and preservation of resolution compared to averaging. Further details of the evaluation of stents and its interaction with blood using ssDOCT will be presented in the next chapter. 

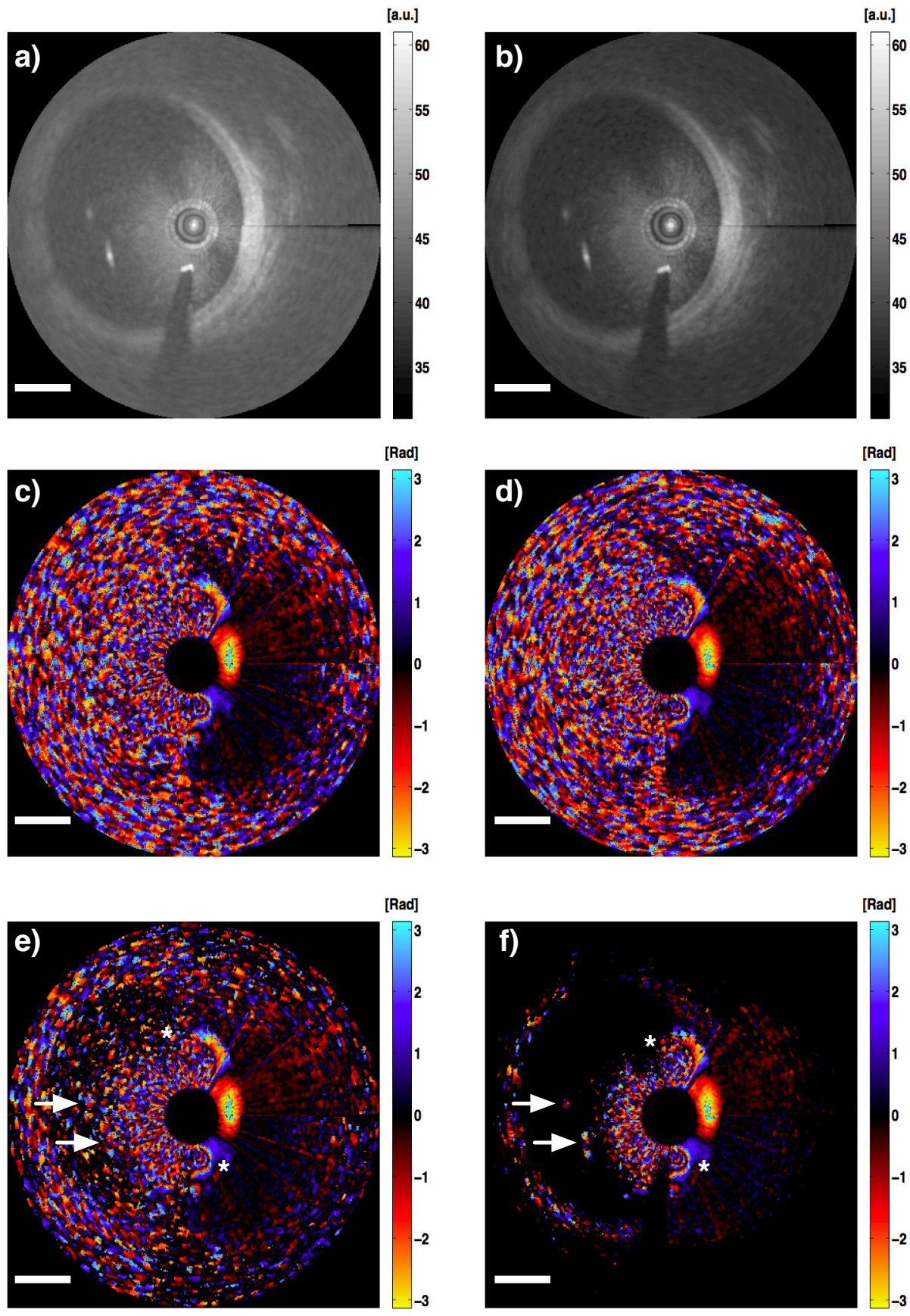

Figure 2.14: (a) Structural image of a deployed stent in the carotid artery using the full length apodization window $(\mathrm{B}=1, \mathrm{M}=4, \mathrm{~N}=20)$. (b) The corresponding structural SSP-OCT image $(\mathrm{B}=2, \mathrm{M}=1, \mathrm{~N}=20$, FBW $=60 \%)$. (c) and (d)The corrected Doppler image computed from DOCT and ssDOCT techniques, respectively. (e) DOCT and (f) ssDOCT were thresholded at the same phase value. Note the two detected flow profiles (upper $* \&$ lower $*)$. The upper flow profile $(*)$ may be caused from a stent strut upstream from the location of the OCT struts. Scale bar $=1 \mathrm{~mm}$ [4]. (Reprinted with permission of the Optical Society of America) 


\section{Chapter 3}

\section{Application of split spectrum Doppler optical coherence tomography in carotid stenting}

The techniques developed in Chapter 2 include the implementation of split spectrum Doppler optical coherence tomography on swept source OCT systems. This chapter highlights the contributions made using these techniques to a clinical problem. In particular, the application of ssDOCT to monitoring stent deployment and visualizing blood flow patterns in the treatment of atherosclerotic plaques is observed. The methods and metrics developed in this chapter can then be translated to the treatment of intracranial aneurysms.

The work presented in this chapter has been published in the following journal.

1. Vuong, B., Genis, H., Wong, R., Ramjist, J., Jivraj, J., Farooq, H., Sun, C., Yang, V.X.D. (2014) Evaluation of flow velocities after carotid artery stenting through split spectrum Doppler optical coherence tomography and computational fluid dynamics modeling. Biomedical Optics Express 5:4405-4416. (PhD Work)

\subsection{Clinical problem of carotid stenting}

Stroke is a leading cause of death (15,000 patients/year) and disability (300,000 patients/year) in Canada [64], resulting in a $\$ 3.6$ billion CDN price tag to the Canadian economy [65]. Atherosclerotic lesions in the intracranial arteries and the resulting stenosis can contribute to the onset of ischemic stroke [66]. Carotid endarterectomy (CEA) is a vascular surgical procedure and was traditionally the standard of care for both patients with symptomatic and asymptomatic high-grade carotid stenosis [67]. Carotidartery stenting (CAS) initially emerged as a minimally invasive alternative, specifically for patients with an increased risk for undergoing CEA. In recent years, CAS has been increasingly performed due 
to its decreased procedural length and patient-recovery times [68]. Recent randomized clinical trials comparing the outcomes of CEA versus CAS have determined both are clinically effective, although each demonstrates individual benefits and complications. The results presented in the CREST (Carotid Revascularization Endarterectomy versus Stenting Trial) study [22] found that stenting results were slightly better for patients aged 69 and younger, with increasing benefit as age decreased. Conversely, for patients older than 70, surgical results were slightly superior to stenting, with the benefits increasing along with the age [69]. Furthermore, endarterectomy poses an increased risk of myocardial infarction while stenting results in greater risk of stroke [22]. Endovascular treatment holds additional concerns, such as the further narrowing of the arterial lumen, known as restenosis, following the procedure. This complication may arise due to the alterations in blood velocities and overall hemodynamic patterns following stent implantation [70]. As a result, it is critical to determine the effect of stent placement in vivo during the endovascular procedure to reduce the risk of peri- and post-operative complications. Carotid stenting results in significant alterations to the treated artery in terms of the vascular morphology and hemodynamics. Although the exact mechanism involved in the development of restenosis is unknown, irregular hemodynamic patterns have been correlated with intimal thickening. Stent implantation can cause non-laminar flow [71]. Specifically, recirculation zones may form near the stent struts, increasing platelet residence time and allowing subsequent aggregation [72]. In addition, stent malapposition can also lead to platelet aggregation and progressive thrombus formation [73]. Further complications also include plaque prolapse through the stent into the arterial lumen, which can induce cerebral embolization [73]. Unfortunately, there is no standard intraoperative technique to identify these micro-defects within the carotid artery.

In the previous chapter, we have demonstrated that ssDOCT provides further suppression of phase noise without incorporating external bulk optical devices. In this chapter, velocity estimates of a bifurcated junction within the subclavian artery (SA) and common carotid artery (CCA) of the porcine model was evaluated with ssDOCT processing before and after stent implantation. Changes in the hemodynamics due to stent placement in the vessels are presented. CFD models was created to support the analysis of ssDOCT provided additional evaluation of a bifurcated junction within the vessel.

\subsection{In vivo split spectrum Doppler optical coherence tomogra- phy imaging during carotid stenting}

Three-porcine models were utilized in this study. The protocol outlined in Chapter 2 for guiding the intravascular OCT catheter was the same. Fluoroscopy and volumetric OCT images were first acquired to locate a side branch in the carotid or subclavian artery of the porcine model. This anatomical location best simulates a bifurcated human carotid artery. After the region of interest was found, ssDOCT images were collected at the bifurcation. The stent was guided by fluoroscopy (Figure 3.1) to the approximate site of the OCT image and was deployed. Similar to the previous chapter, a blood and saline mixture was injected in order to measure the Doppler frequency shifts.

Before stent implantation, the ssDOCT had measured high velocities at the neck of the bifurcation 

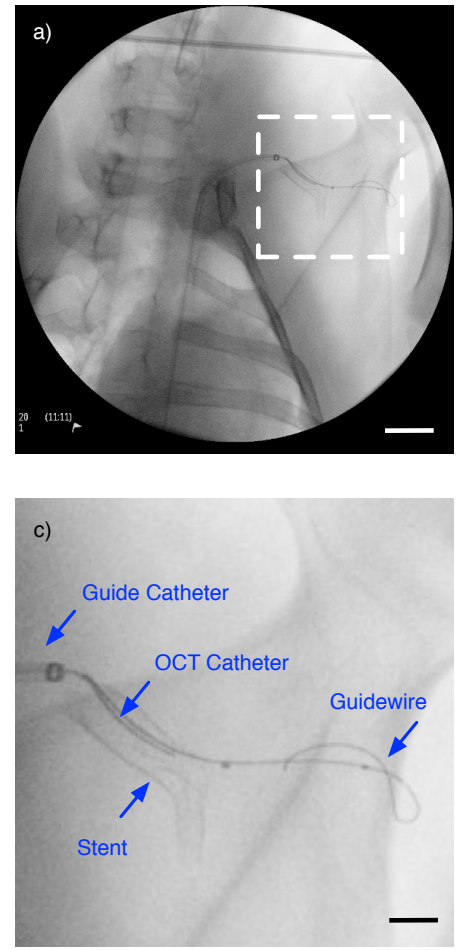
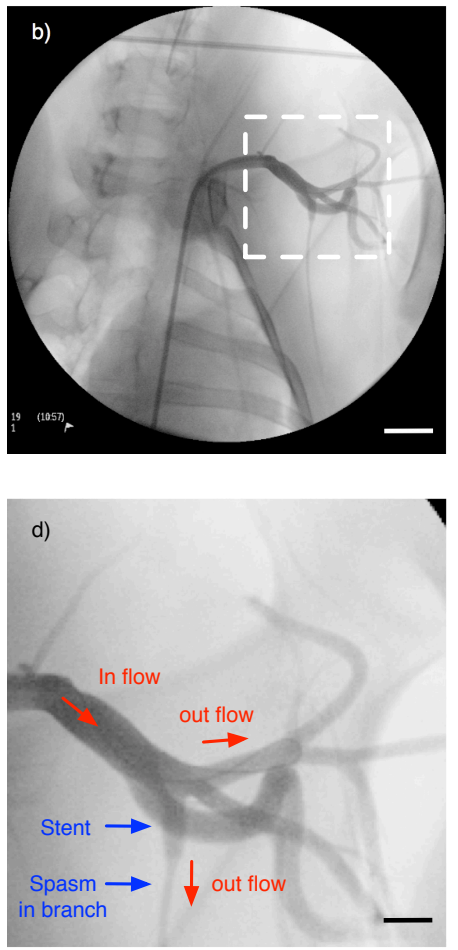

Figure 3.1: (a) Fluoroscopic image of the stent placement. (b) Contrast was injected into the artery in order to visualize the gross flow distribution of the vessel after stent intervention. Scale bar 10mm. (c) Magnified view of the region marked with the dashed box in (a), showing details of catheter and guidewire positions. (d) Magnified view with contrast injection, showing blood in-flow, mild spasm distal to the stent edge in an out-flow branch. Notice in this particular setting, the OCT imaging catheter and its guidewire are placed through the stent in one of the out-flow branches. Scale bar: $3 \mathrm{~mm}$ [5]. (Reprinted with permission of the Optical Society of America)

(Figure 3.2). An increase in blood concentration was observed at the neck of the side branch resulting in unresolvable velocity estimates. Distal to that location and within the parent vessel, lower velocity (fewer phase contours) was visualized.

After stent implantation, ssDOCT images revealed several different hemodynamic flow changes to the vessel. Stent malapposition was observed as unanchored stent struts on the vessel wall left a pocket where blood can flow through (Figure 3.3(a)). The hemodynamic flow pattern differentiated when the stent struts were placed on the vessel wall (white dashed box). It can be seen that the Doppler phase profile enveloped the single strut for a portion of the time without significant disruptions. However, dense stent strut spacings at the 5 O'clock portion demonstrated low velocities at the vessel wall, while exhibiting minimal perturbation of flow.

Other flow patterns were seen at the stented bifurcation (Figure 3.4). Low signal-to-noise Doppler phase shifts could be seen at the neck of the bifurcation, where the edge of a protruded stent strut had occurred. The phase contours extended beyond the stent strut and along the vessel wall of the side branch. In Figure 3.4(b), Doppler phase had enveloped around the protruded stent strut. By evaluating 
a)

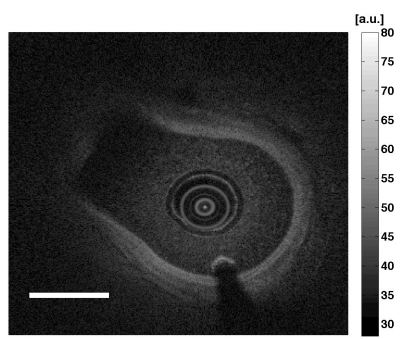

b)

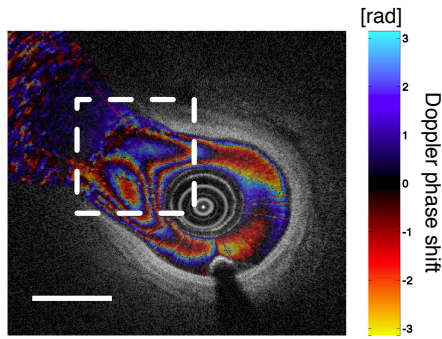

c)

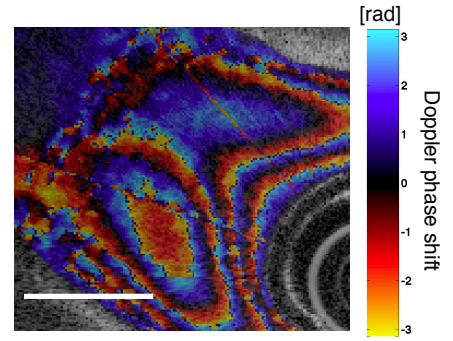

Figure 3.2: (a) In vivo structural OCT image of the carotid artery before stent deployment. (b) The corresponding ssDOCT image. Scale bar: $1 \mathrm{~mm}$. (c) Magnification at the neck of the carotid artery (dashed box). Scale bar: $0.5 \mathrm{~mm}$. [5]. (Reprinted with permission of the Optical Society of America)
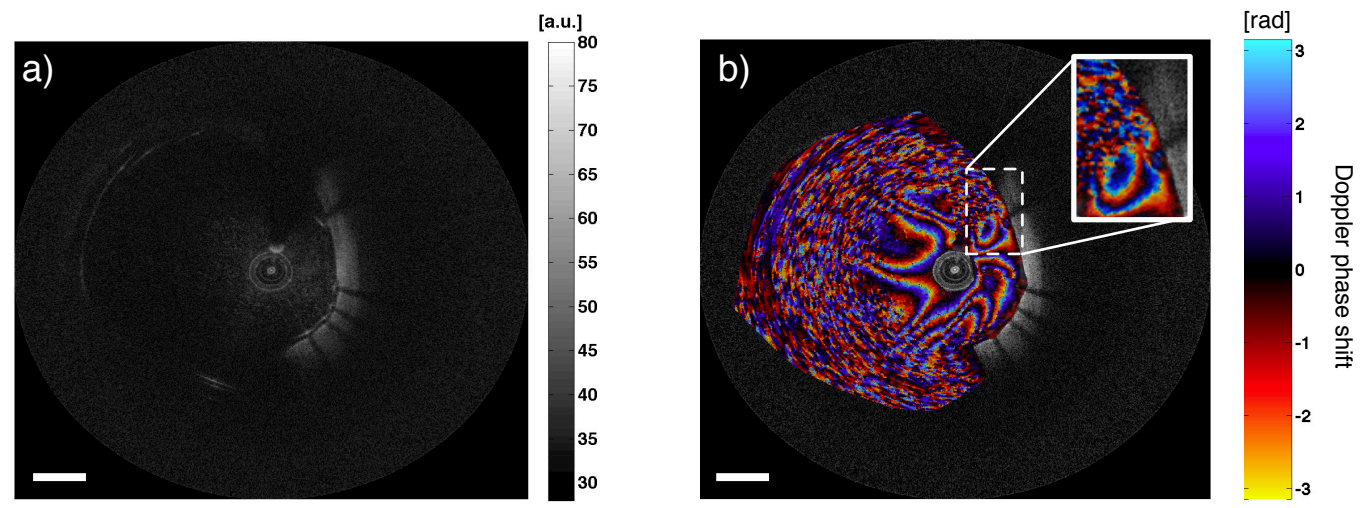

Figure 3.3: (a) In vivo structural OCT image of the ICA after stent implantation. (b) The corresponding ssDOCT image. A magnification of the ssDOCT image revealed phase contours that envelope the stent struts. Scale bar: $1 \mathrm{~mm}$ [5]. (Reprinted with permission of the Optical Society of America)

the Doppler phase contour over several frames, an eddy current was believed to have formed around the struts in the ssDOCT image (Figure 3.4(d)). Over time the eddy current dissipated, leaving low to stagnant regions.

Low to stagnant regions were primarily observed in the ssDOCT images of a stented ICA. Stagnant to low velocity, as well as low wall shear stress (or endothelial shear stress) areas, have been associated with increased formation of thrombus [74]. This formation is due to the accumulation of fibrin which activates platelets leading to thrombogenesis $[72,75]$. Further supporting evidence have suggested that the presence of low wall shear stress and contributes to stent restenosis [74]. Malappositioned stent struts could interrupt the homeostasis of the vessel wall which may be detected by the endothelium of the vessel wall. This stent position triggers vascular remodeling leading to intimal hyperplasia, formation of atherosclerosis or early onset of embolization [72,74,76].

The presence of an enveloped phase contour suggested that there was little perturbation of the 

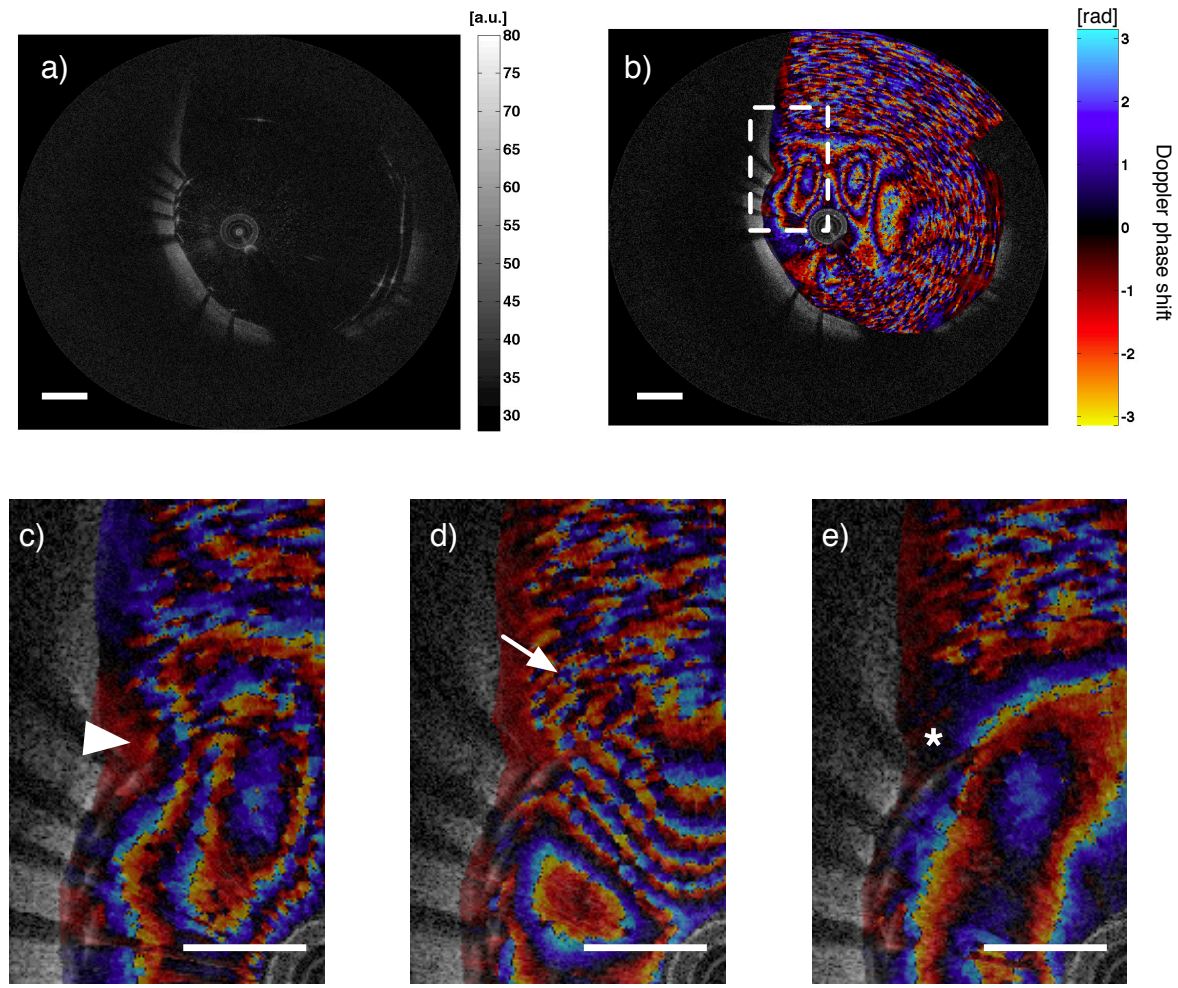

Figure 3.4: (a) In vivo structural OCT image of a protruded stent strut at the neck of the side branch (b) The corresponding ssDOCT image, where the dashed box indicates the protruded stent strut. (c) This magnified region of interest depicts a slow velocity profile (triangle) (d) After 1.25s, it was noted that there was a formation of an eddy current (arrow). (e) At $1.55 \mathrm{~s}$ from the initial frame, low to stagnant velocity measurements were observed $(*)$. Scale bar $1 \mathrm{~mm}[5]$. (Reprinted with permission of the Optical Society of America)

flow and proper placement of the stent. Flow patterns around the stent struts have been extensively studied $[77,78]$. When the stent is properly placed onto the vessel wall, a flow dynamic phenomenon known as backward facing step flow or forward facing step flow occurs [78]. Briefly, a small region between the vessel wall and stent strut forms a separation zone. In this zone, the fluid does not mix with the fluid in the main flow of the artery [78]. These regions also exhibit stagnant flow which could result in the accumulation of thrombi and create other complications including injury to the smooth muscle cells, endothelial denudation, and disrupt the elastic laminae [78]. Furthermore, this enveloped phase contour has also been observed in sparsely positioned stent struts and indicates that the fluid reattaches between the struts [79]. Dense positioning of stent struts often results in recirculation zones or stagnant regions [79].

Another advantage to the utilization of ssDOCT was the observation of the overall local flow patterns in the ICA. Figure 3.5(a) depicts a proper stent placement in a bifurcated ICA, where two struts had bridged the neck of the bifurcation. In the corresponding ssDOCT image, low-velocity laminar flow was 
measured (Figure 3.5(b)). Subsequent frames demonstrate a further decrease in velocity, then a backflow of blood from the side branch to the main branch. This occurrence has not been reported in previous literature. As a result, CFD modeling was utilized to attempt to understand this hemodynamic event.
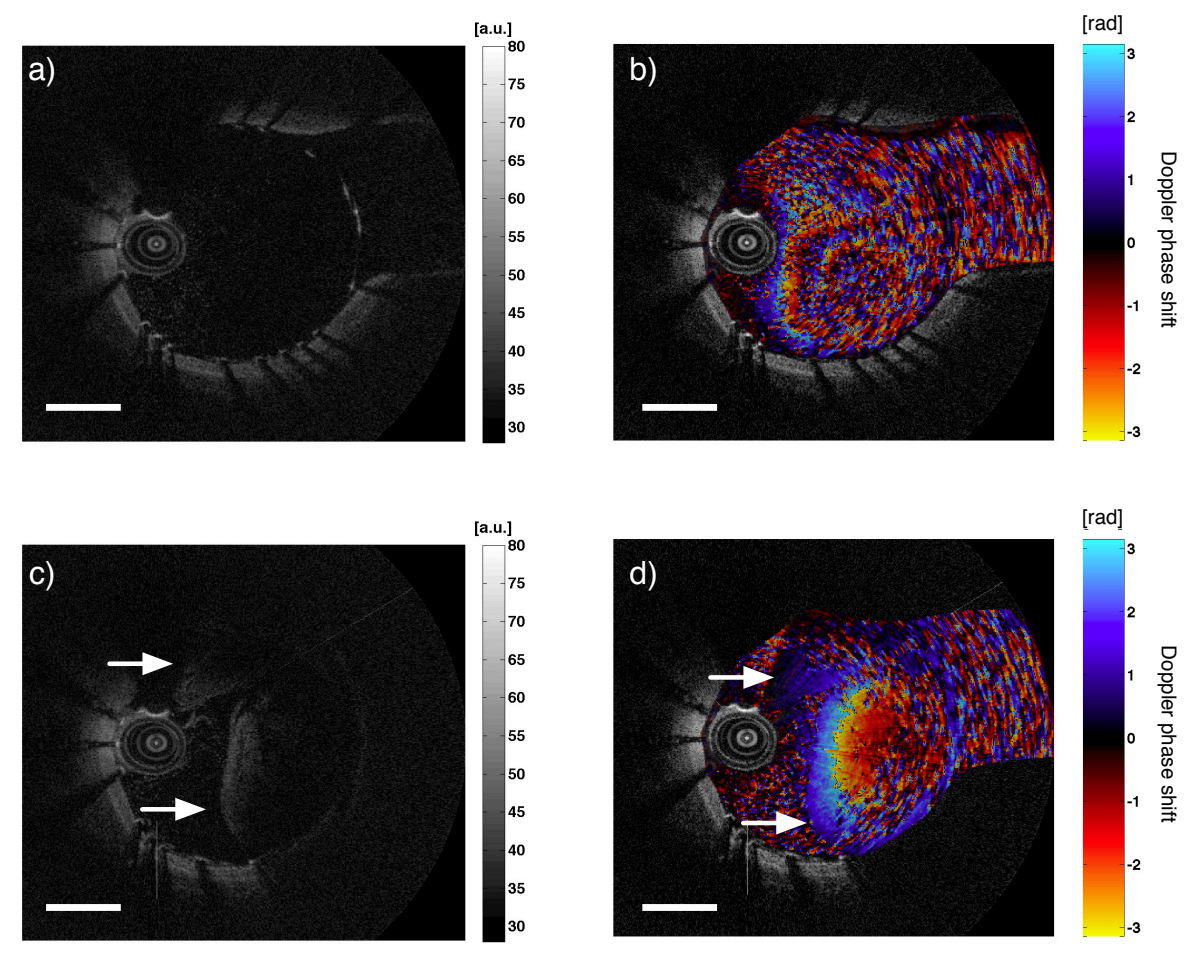

Figure 3.5: (a) In vivo structural OCT image after stent deployment in the ICA. (b) The corresponding ssDOCT image. (c) In vivo structural OCT image of backflow from the side branch to the main branch. (d) The corresponding ssDOCT image of the backflow. Scale bar: $1 \mathrm{~mm}$ [5]. (Reprinted with permission of the Optical Society of America)

\subsection{Computational fluid dynamics of stented carotid artery}

Over the past decade, CFD models have been implicated in various predictions of blood flow in a multitude of virtual arteries and bifurcations [80-86]. The geometry and flow boundary conditions used in these studies are a simplification of the physical vessel. However, the basic blood flow characteristics can play an important role in understanding many vascular diseases.

In this work, the virtual geometry was generated in Solidworks (V2014, Dassault Systèmes SolidWorks Corp., France) based on dimensions of the ICA that were measured using the structural OCT image. The approximate shape and size of the stent used in the in vivo experiments was virtually created and placed near the location of the bifurcation, as observed in the volumetric OCT image. Time-dependent flow analysis of the blood flow was modeled using incompressible Newtonian fluid using a multi-physics 
a)

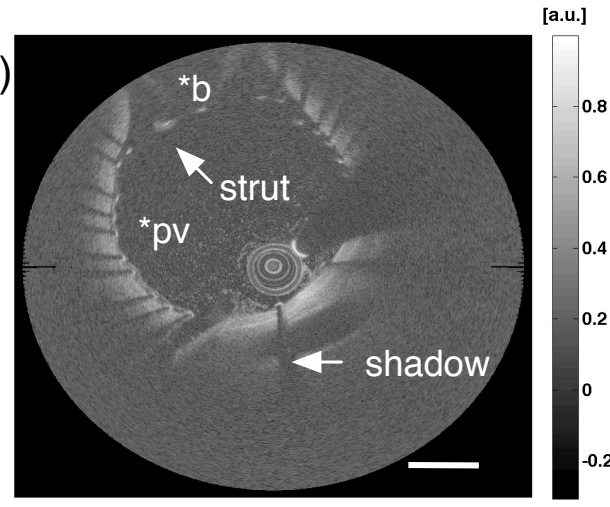

c)

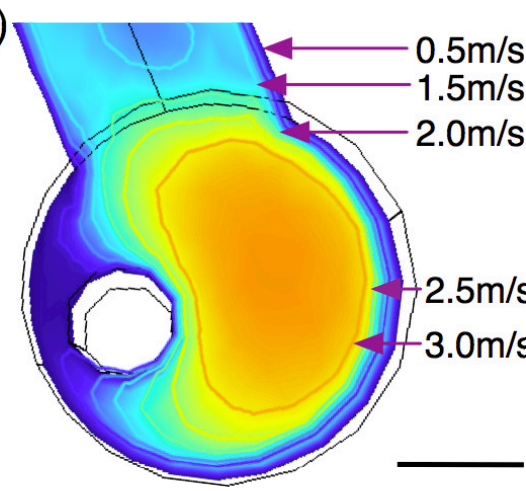

e)
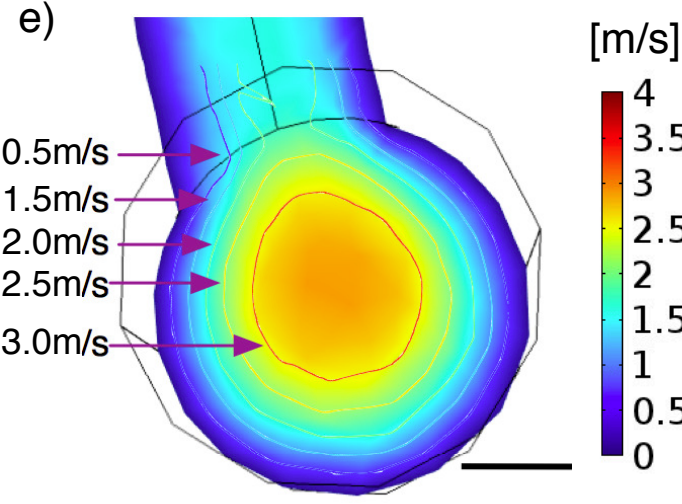

$[\mathrm{m} / \mathrm{s}]$

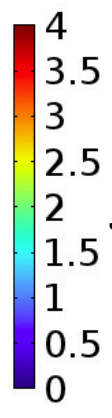

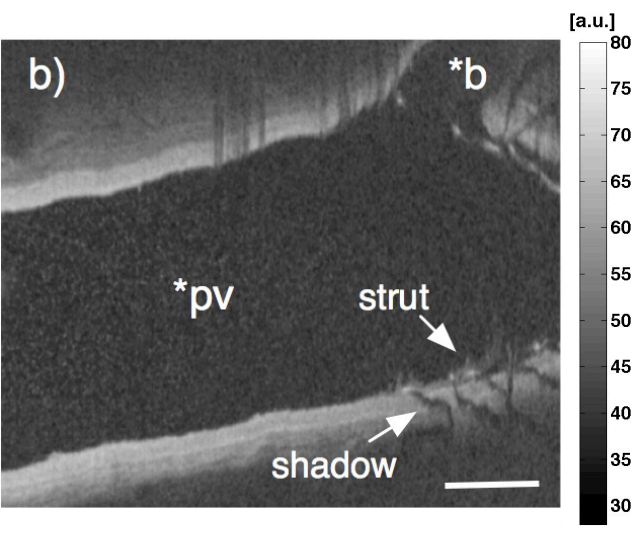

d)

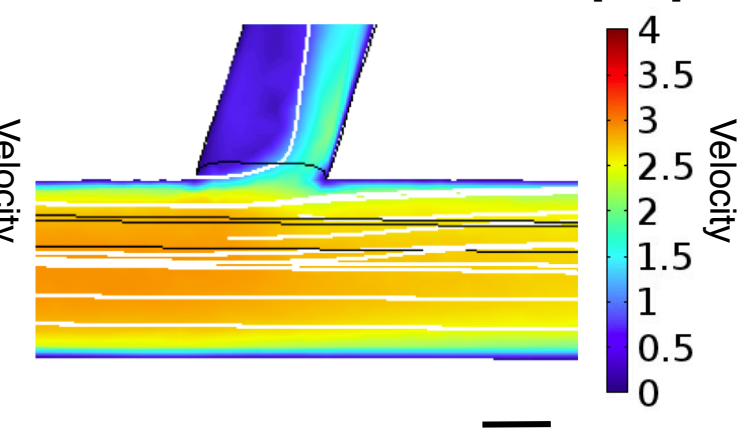

f)

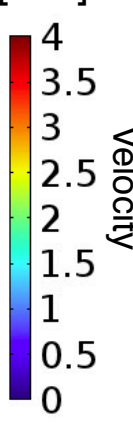

$[\mathrm{m} / \mathrm{s}]$

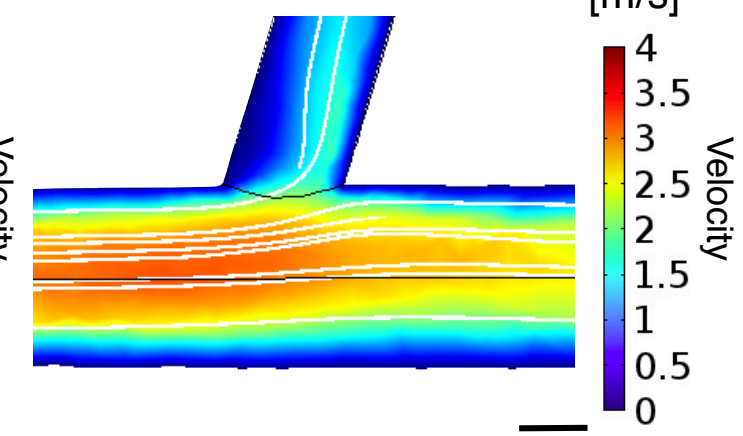

Figure 3.6: (a) Structural OCT image in the transverse plane, demonstrating the parent vessel (*pv), side branch $(* \mathrm{~b})$, and the stent struts with shadows. (b) Reconstructed longitudinal plane of the blood vessel from OCT pull-back imaging was employed as a reference for the 3D geometry of the CFD model. (c) The velocity distribution before stent implantation with the OCT catheter from the CFD simulation of the carotid artery in the transverse plane with flow velocity contours. (d) Streamlines overlaid on the CFD simulated velocity distribution with the OCT catheter in the longitudinal plane. (e) The CFD simulation of the velocity distribution before stent placement without the OCT catheter. (f) The corresponding streamlines that was overlaid on the CFD simulated velocity distribution without the OCT catheter in the longitudinal plane. Scale bar: $1 \mathrm{~mm}$ [5]. (Reprinted with permission of the Optical Society of America) 
simulator (COMSOL V4.4, USA). The fluid dynamic properties of both blood and arterial wall were accounted for during the simulation. Cardiac cycle was modeled after a measured phase contrast MRI waveform from a previously reported human ICA [87]. A peak velocity was selected to be $2.3 \mathrm{~m} / \mathrm{s}$, as previous literature has shown it to be a threshold for severely stenosed ICA [88]. The CFD models were also assumed to have a laminar flow profile and were solved with Navier-Stokes equations.

The flow distribution of the virtual 3-dimensional (3-D) bifurcation with and without the influence of the OCT catheter can be seen in Figure 3.6. Laminar flow was observed in the parent vessel of the simulated ICA while the side branch exhibited diverted flow at the entrance. The eccentrically placed OCT catheter had also resulted in a laminar-like flow in the parent vessel. At the entrance of the bifurcation, higher velocities proximal to the carina (junction point of the bifurcation) and low to stagnant velocity regions distal to the carina were demonstrated both with and without OCT catheter placement. The consistent high-velocity streams that flowed through the neck were also visualized in the ssDOCT image. From the transverse plane (cross-sectional view) of the CFD model, it was noted that there was a consistent flow at the neck of the side branch which then gradually decreased in velocity as blood flowed away from the neck of the side branch.

Following virtual stent implantation, flow distributions within the arterial vessel were significantly altered. More specifically, the space between each of the stent strut at the neck of the side branch exhibited velocity slipstreams with and without the presence of the OCT catheter. In both scenarios, formation of eddy currents were observed near the stent struts that were located at the entrance of the side branch (Figure 3.7(a) and Figure 3.7(b)). At approximately $1.5 \mathrm{~mm}$ distal from the carina within the side branch, stagnant flow regions were observed. Additionally, a laminar like profile with increased velocity in the parent vessel was observed with the introduction of the OCT catheter. Figure 3.7(a) and Figure 3.7(b) demonstrated low-velocity flow patterns, where overlaid streamlines had enveloped around the strut. Contralateral to the placement of the OCT catheter, recirculation or vortices had formed in Figure 3.7(c) at various stent strut locations. These recirculation zones were also observed without the influence of the OCT catheter. This CFD model had agreed with the ssDOCT image shown in Figure 3.4(d), where isolated eddies were believed to have occurred around the protruded stent strut from the entrance of the side branch. However, in regards to the backflow from the side branch into the main branch, the CFD model was not able predict this hemodynamic event. It was noted that this rare phenomenon might only occur under specific pressure and velocity conditions. Unfortunately, due to the simplification of the CFD modeling of the in vivo experiment, we were unable to recreate this phenomenon.

Other limitations included the construction of the virtual stent, which used a generic strut pattern. The design of the stent struts (diameter, length and the thickness) was an approximation of the stent used in the in vivo porcine experiment. Furthermore, the position of the stent relative to the entrance of the side branch was approximated using the volumetric OCT image. However, these approximations would significantly affect the flow slipstreams, as extensive studies have shown that the shape of the stent struts, its spacing and its position are critical stent parameters [89]. This stent design and position approximation may have resulted in the discrepancies between the ssDOCT images and CFD model.

It should also be noted that ssDOCT imaging consisted of the injection of a blood \& saline mixture 
in order to provide both visualization of the lumen wall and scattering for Doppler phase estimates. This would reduce the local viscosity of the blood flow and alter its hemodynamics. This was not considered during the CFD simulations and further investigations of time varying viscosity should be explored in future studies. Furthermore, the hemodynamic influence of the OCT catheter within the lumen is still not well understood. Control flow chambers would be necessary to evaluate its influence in the Doppler imaging. Finally, the inlet velocity was assumed to exhibit a peak velocity that is consistent with a stenosed ICA. The velocity in a healthy ICA is between $20 \mathrm{~cm} / \mathrm{s}$ to $112 \mathrm{~cm} / \mathrm{s}$ [90]. Unfortunately, the inlet velocity was not measured during the experiments. Furthermore, the CFD image in Figure 3.7 was simulated at peak systole (Black arrow in Figure 3.8(a)). When ssDOCT images are acquired at systole, the images are completely aliased. In this study, phase shifts were resolved during the diastole portion of the cardiac cycle (Green arrow in Figure 3.8(a)). It is speculated that the measurement at different portion of the cardiac cycle had resulted in the large discrepancy seen between the CFD and ssDOCT.

Despite these approximations, similarities between the ssDOCT images and CFD were observed. Figure 3.8 demonstrates further correlation between CFD and ssDOCT. In this case, the inlet velocity was assumed to be $20 \mathrm{~cm} / \mathrm{s}$ and the stent was neglected for simplification. The peak velocity in the ssDOCT was estimated to be approximately $13.4 \mathrm{~cm} / \mathrm{s}\left(\right.$ Doppler angle $\left.=80^{\circ}\right)$. This measurement correlates well with the CFD, which had estimated a peak velocity of $14 \mathrm{~cm} / \mathrm{s}$ The results could provide metric for surgeons to assess the quality of the stent placement in order to reduce post-operative complications. This same feedback could be used in stenting or coiling of intracranial aneurysms. In the next chapter, we applied similar ssDOCT imaging and CFD simulations in patient-specific aneurysm models. 
a)
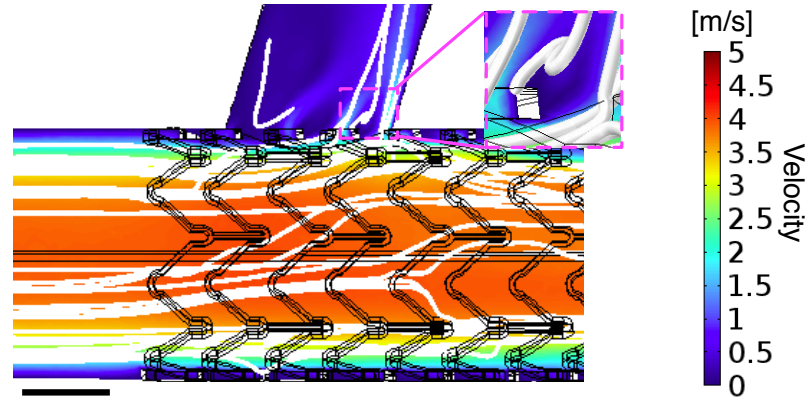

b)

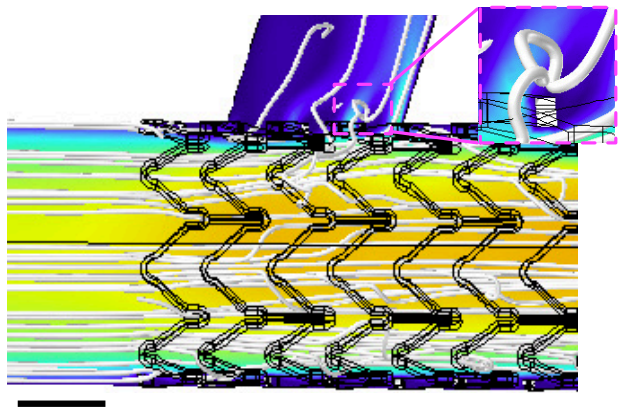

$[\mathrm{m} / \mathrm{s}]$

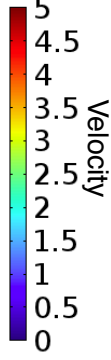

c)

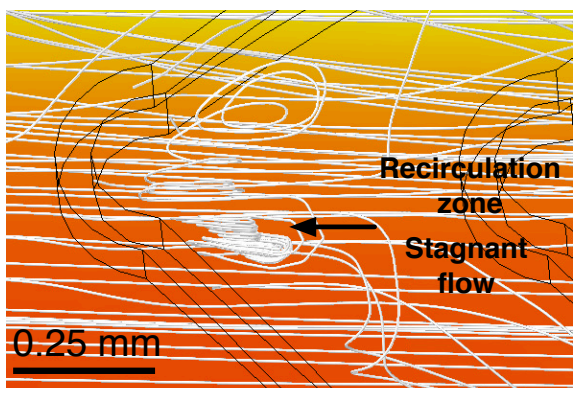

$[\mathrm{m} / \mathrm{s}]$
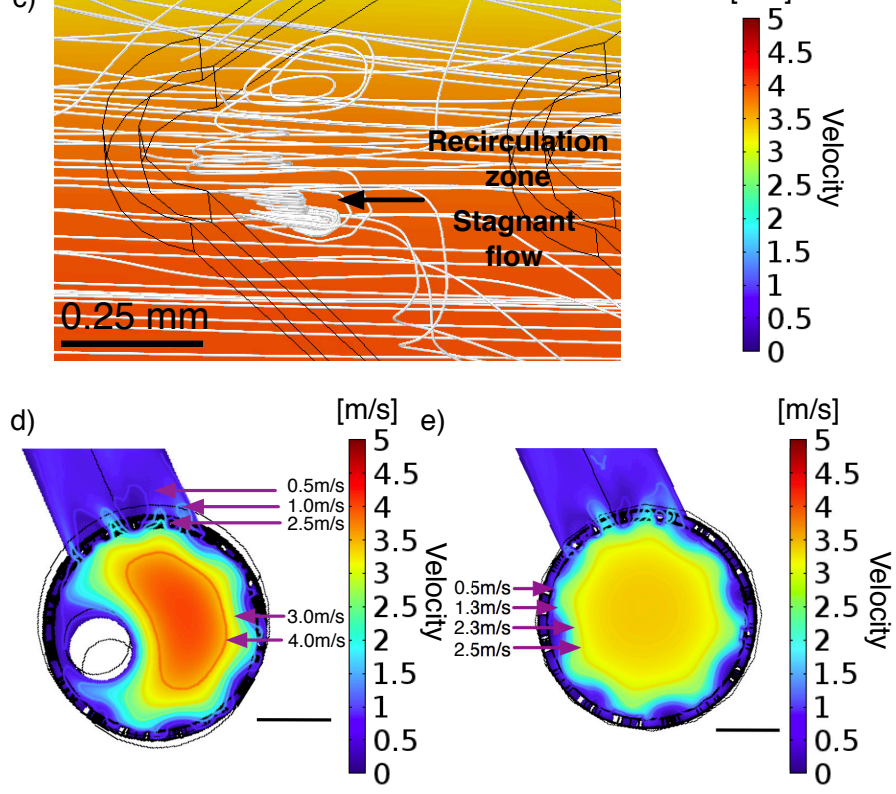

Figure 3.7: (a) The velocity distribution from the CFD simulation with the OCT catheter present after stent implantation covering the bifurcation in the longitudinal plane. Streamlines were overlaid onto the velocity distribution. An eddy was observed close to the stent strut near the entrance of the bifurcation (dashed region). (b) The corresponding CFD simulation without the OCT catheter within the lumen wall. Similarly, an eddy current was also observed around the stent strut near the entrance of the bifurcation (dashed region) (c) Contralateral to the position of the OCT catheter, a recirculation zone near a stent strut was observed. (d) The CFD simulation was re-sliced to view the distribution in the transverse plane with the OCT catheter. (e) The corresponding CFD simulation without the OCT catheter image. Scale bar: $1 \mathrm{~mm}$ [5]. (Reprinted with permission of the Optical Society of America) 
a)
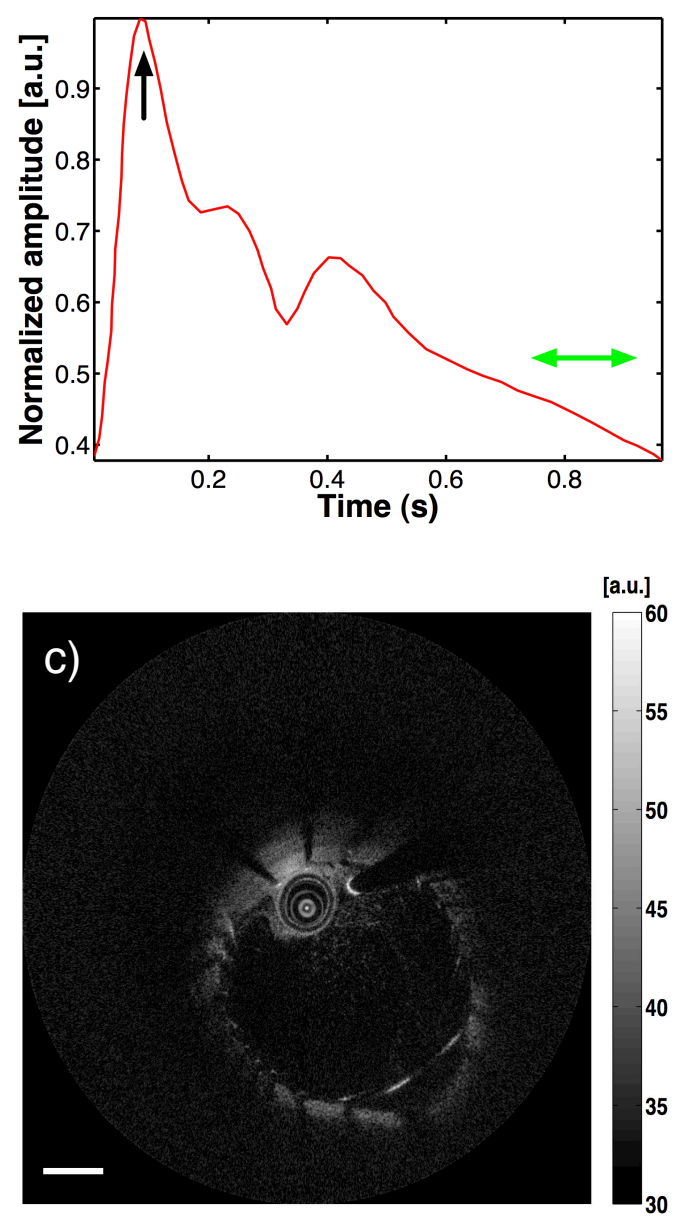

b)
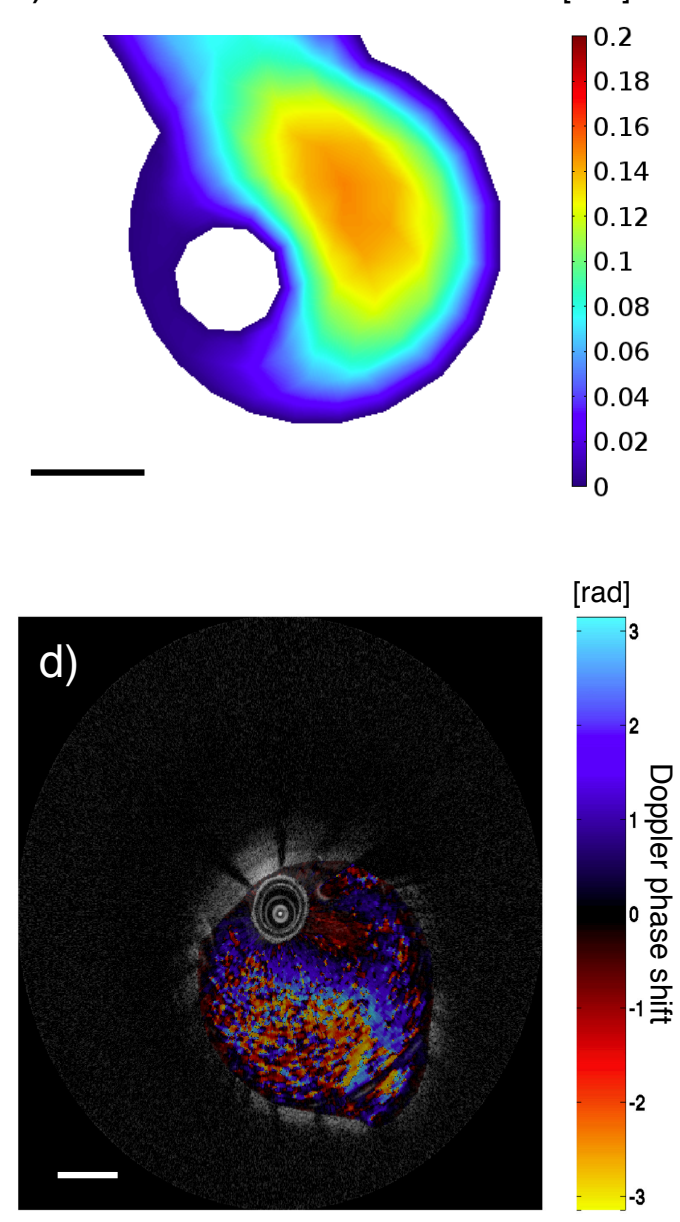

Figure 3.8: (a) The normalized inlet velocity profile used during CFD modeling. Peak velocity (black arrow) was simulated in Figure 3.7, while ssDOCT were observed during late diastole (Green arrows) (b) The cross section of the CFD with an inlet velocity of $20 \mathrm{~cm} / \mathrm{s}$ (c) Structural OCT image when the OCT catheter was place distally near the carina (d) The corresponding ssDOCT image. Scale bar: 1 $\mathrm{mm}$. 


\section{Chapter 4}

\section{Application of split spectrum Doppler optical coherence tomography in intracranial aneurysm flow phantoms}

The progression of the work presented in Chapter 2-3 has resulted in the ability to measure velocity in the ICA. This chapter demonstrates the potential application of intravascular ssDOCT imaging within an intracranial aneurysm using a flow phantom. To our knowledge, this is the first study that demonstrates Doppler OCT imaging of a modeled intracranial aneurysm.

The work presented in this chapter has been submitted to the following journal.

1. Vuong, B., Ramjist, J., Genis, H., Wong, R., Jivraj, J., Jakubovic, R., Keith, J., Kiehl, T.R., Yang, V.X.D. (2015) Evaluation of hemodynamics in intravascular aneurysms through split spectrum Doppler optical coherence tomography and computational modeling techniques. American Journal of Neuroradiology. (PhD Work) (Submitted)

\subsection{Intracranial aneurysm flow phantoms}

Recently, the C7-XR intravascular OCT system was used for cadaveric imaging to evaluate deployment of embolization devices in the circle of Willis [91]. Difficulty in the advancement of the OCT catheter through the carotid siphon was reported. As a result, it is unknown if the current state of the art OCT catheter is suitable or safe for imaging of intracranial aneurysm. In addition, there has also been a significant amount of literature that utilized patient specific CFD modeling. These studies have demonstrated the ability to estimate the flow dynamics within intracranial aneurysms through MRI, 
CTA, and flow phantoms to investigate the factors that contribute to the rupture of an intracranial aneurysm [92-94].

In terms of the treatment of a wide neck or large intracranial aneurysm, the common standard of care is the utilization of a stent and coils. However, these interventions have been associated with possible thromboembolic complications [95]. Similar to coronary and carotid complications following stent implantation, the factors that contribute to thromboembolic events are not well understood and can cause significant neurologic deficits. As a result, this study investigates the hemodynamics associated with intracranial aneurysms using intravascular OCT catheters.

\subsubsection{Simplified intracranial aneurysm flow phantoms}

Initial ssDOCT imaging was performed on a silicone based elliptical shaped intracranial aneurysm phantom flow model with an outer diameter of $13 \mathrm{~mm}$ (width) x $10 \mathrm{~mm}$ (height) x $8 \mathrm{~mm}$ (depth). An adjustable fluid injection pump coupled to a 3-way stop connector which pulsed intralipid fluid at approximately 60 beats per minute into the phantom. The outlet port siphoned the intralipid into a reservoir from the fluid injection pump. Insertion of the OCT catheter was a simplified protocol of the previously outlined protocol for stent deployment during the in vivo porcine model study discussed in Chapter 3. Briefly, a guide-wire was inserted in the available port and positioned near the neck of the elliptical aneurysm. The OCT imaging catheter was advanced using a monorail rapid exchange technique (Figure 4.1a).
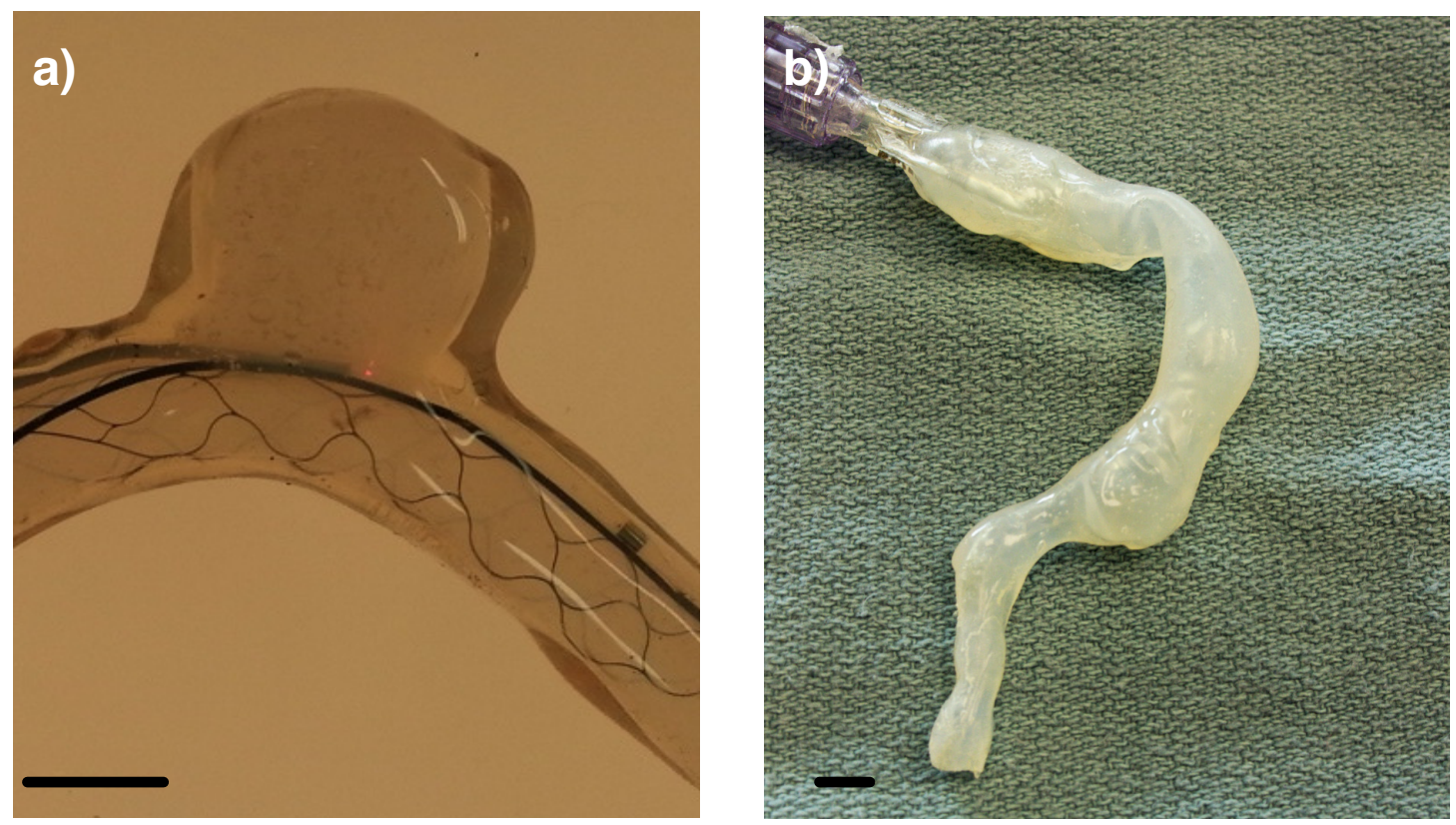

Figure 4.1: (a) Photo of the stented simplified intracranial aneurysm flow phantom. (b) Photo of the patient specific intracranial aneurysm flow phantom. Scale bar: $5 \mathrm{~mm}$ 


\subsubsection{Patient specific intracranial aneurysm flow phantom}

The patient specific intracranial aneurysm flow phantom consisted of a silicone lumen wall (Figure 4.1b). This model was derived from a 63-year-old female (Figure 4.2) exhibiting a symptomatic right posterior cerebral artery aneurysm (PCA). CTA assessment of the aneurysm had indicated a diameter of $12 \mathrm{~mm}$ (pre-operative). After the placement of a flow diverting embolization device in the PCA, the patient's health degraded and she passed away. Consent was obtained from the patient's family for autopsy, histological evaluation (CVpath Institute Inc.) and medical images. The 3-D angiogram of the PCA was used for the fabrication process and the CTA data set was employed to verify the correct scaling. The inlet of the flow phantom models was coupled to an electronically controlled fluid injection pump, which pulsed the intralipid fluid at a rate of $\sim 60$ beats per minute.

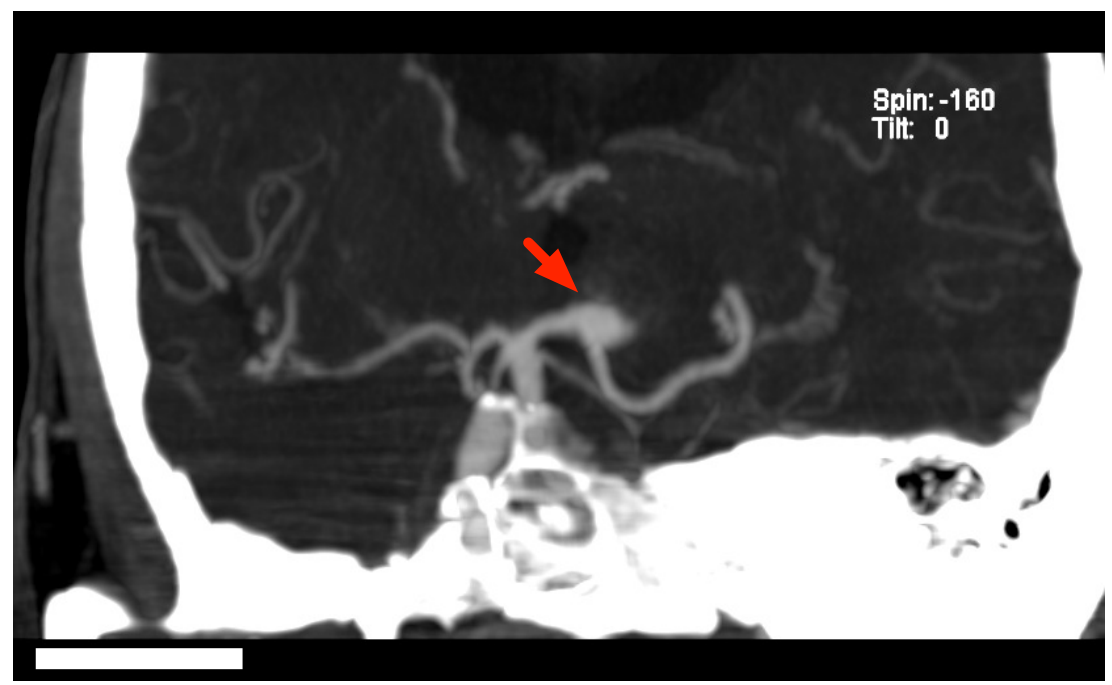

Figure 4.2: A CT image of a 63-year-old female with a PCA aneurysm. Scale bar: $5 \mathrm{~cm}$.

\subsection{Split spectrum Doppler optical coherence tomography of an intracranial aneurysm flow phantom.}

\subsubsection{Imaging of a simplified intracranial aneurysm flow phantom}

During the diastolic portion (Pump ON to OFF) of the injection pump cycle, mid to low velocities in both the parent vessel and the neck of the aneurysms were observed in the pre-stent implantation. Peak velocities at the parent vessel were consistently higher than velocities at the neck. During systolic flow, the velocities estimates in the parent vessel could not be resolved due to the aliasing of high velocity flow (Figure 4.3(a) and Figure 4.3(b). At the neck of the aneurysm, lower velocities were observed distally from the parent vessel. The asymmetric contours exhibited by both the parent vessel and neck suggest a non-laminar profile. 

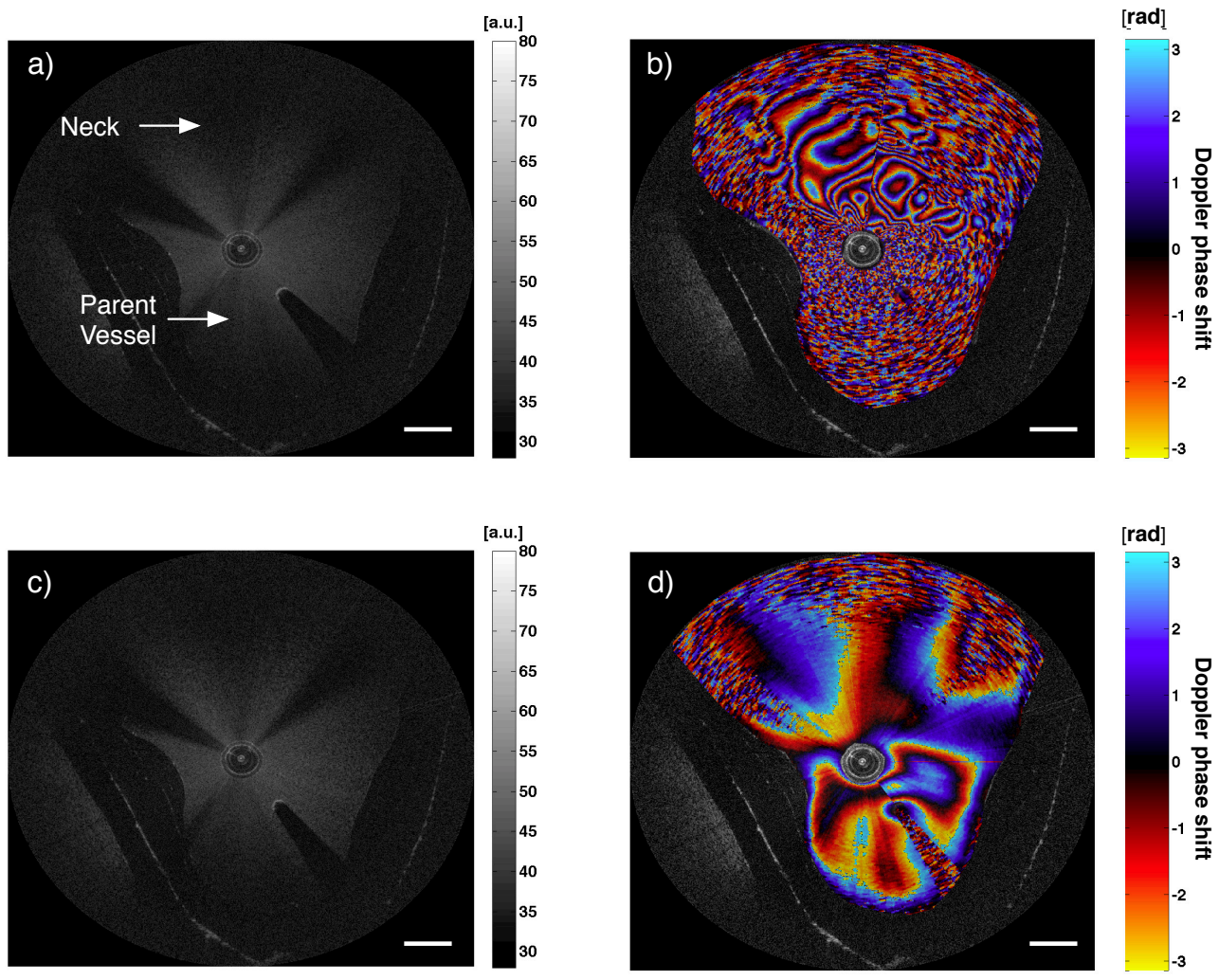

Figure 4.3: (a) OCT structural image of a simplified aneurysm model before stent implantation during the systolic cycle. (b) The corresponding ssDOCT image. Low velocities estimates were resolved at the neck of the aneurysm. (c) OCT structural image of a simplified aneurysm during the diastolic cycle. (d) The corresponding ssDOCT image. Majority of the flow profiles were mid to low velocities. Scale bar: $1 \mathrm{~mm}$.

Figure 4.4(a) to Figure 4.4(d) depicts the diastolic and systolic portion of a stented aneurysm phantom. Similar to the pre-stented aneurysm, the parent vessel also exhibited higher velocities than the neck of the aneurysm during the diastolic cycle. At the end of the systolic peak, lower velocities were observed at the neck of the aneurysm. This differed from the pre-stented implantation as the visualization of velocities distal to the parent vessel were more difficult to resolve throughout the entire systole period. At the neck of the aneurysm, several contour lines were observed, which is indicative of a high velocity region. During diastolic flow, laminar-like flow was seen within the parent vessel, while uniform flow was noted at the neck of the aneurysm flow model.

\subsubsection{Imaging of patient specific intracranial aneurysm flow phantom}

Similar to the simplified elliptical intracranial aneurysm flow phantom imaging, ssDOCT images were acquired in a patient-specific flow phantom during the diastolic (Figure 4.5(a) and Figure 4.5(b)) and 

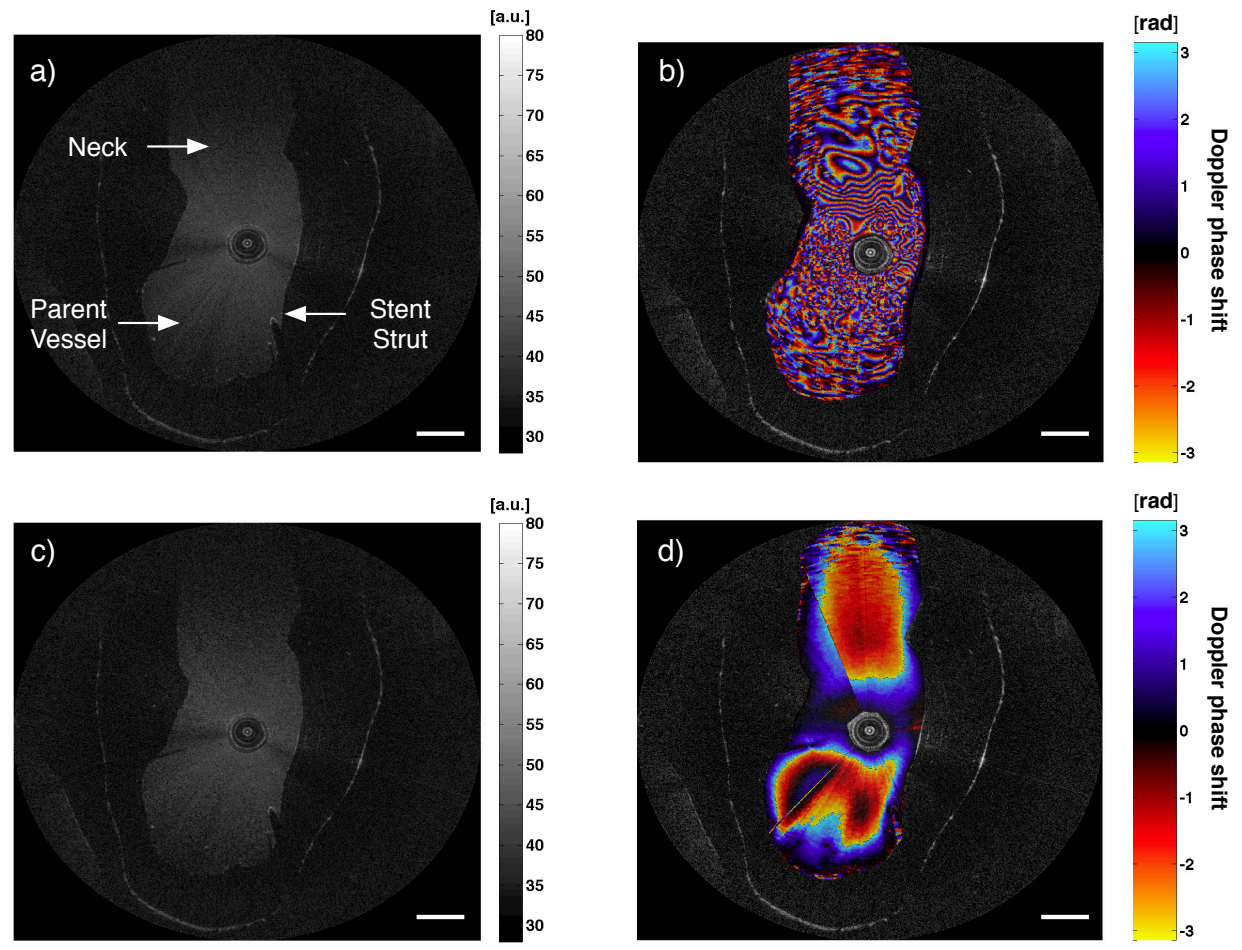

Figure 4.4: (a) OCT structural image of a simplified aneurysm model after stent implantation during the systolic cycle. (b) The corresponding ssDOCT during systolic cycle. Low velocities were observed at the neck of the aneurysm. (c) The structural OCT image during diastolic cycle. (d) The corresponding ssDOCT during diastolic cycle. The laminar-like profile was observed in the neck of the aneurysm, whereas the parent vessel exhibited asymmetric flow profile. Scale bar: $1 \mathrm{~mm}$.

systolic (Figure 4.5(c) and Figure $4.5(\mathrm{~d})$ ) portions of the injection pump cycle. At the diastolic phase, ssDOCT measured two distinct flow regions. In color Doppler ultrasound, particles moving away from the transducer are represented by a blue region, while particles moving towards the transducer are indicated by a red region. This color Doppler ultrasound algorithm is similar to ssDOCT which demonstrates two flow regions that are flowing in opposite directions in Figure 4.5(b). Separation of contours (dashed line) was also observed during the systolic ssDOCT image (Figure 4.5(d)), which indicates two districts flow regions but at higher velocity (multiple phase contours) compared to the diastolic ssDOCT image.

\subsection{Computational fluid dynamic models of intracranial aneurysm}

Much like the CFD models that evaluated the carotid artery, the elliptical shaped aneurysm was generated in Solidworks. The geometry of the patient specific artery wall was also generated from 3D angiography and CTA. Both these models were imported into COMSOL. The visualized arteries were assigned inlet and outlet in the simulation, where the flow of blood had a viscosity of $0.004 \mathrm{~Pa} \cdot \mathrm{s}$ and 

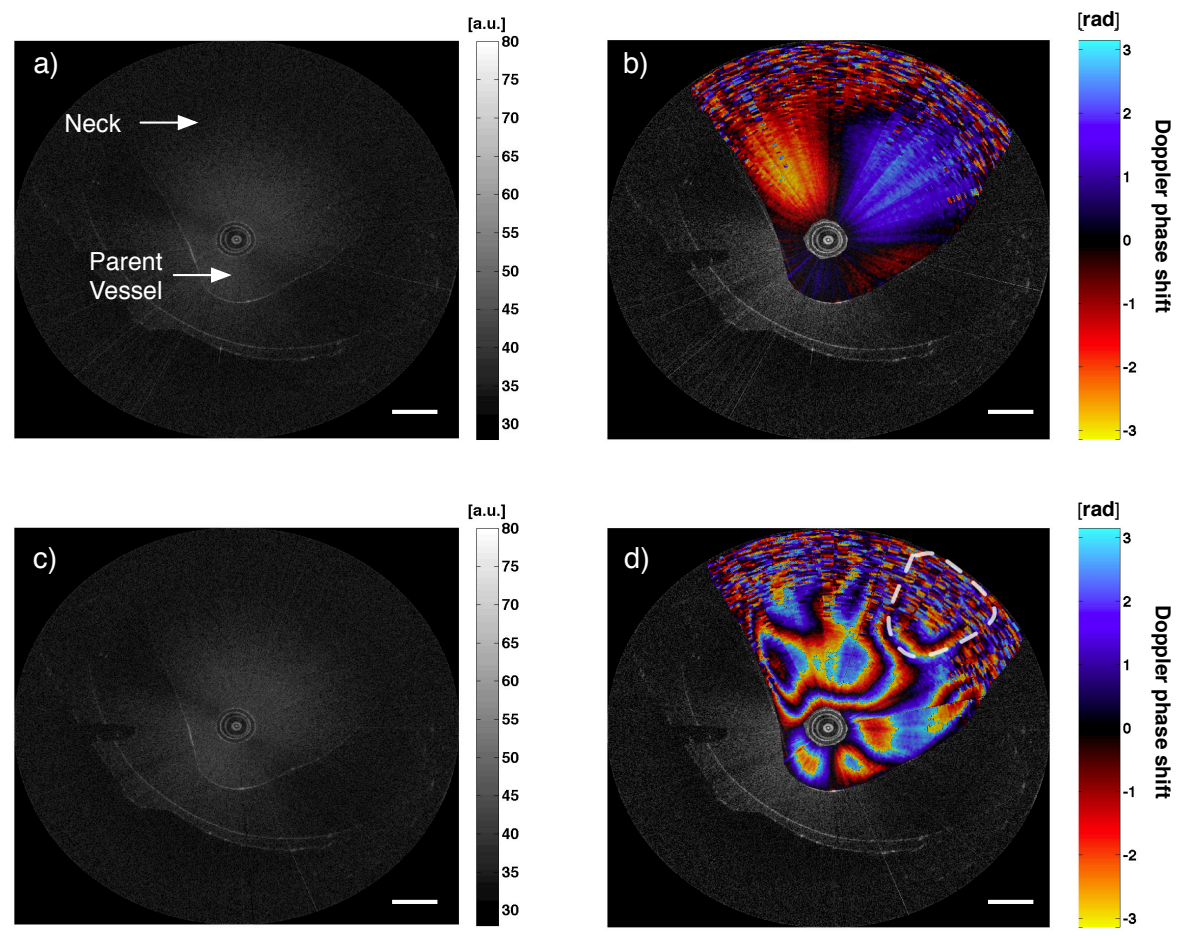

Figure 4.5: (a) Structural OCT image of a patient-specific flow phantom during the diastolic flow. (b) The corresponding ssDOCT image. Two distinct flow regions were observed. (c) Structural OCT image of a patient-specific flow phantom during the systolic flow. (d) The corresponding ssDOCT image demonstrated higher flow velocity (increased phase contours). Separation of flow contours was also observed (dashed line). Scale bar: $1 \mathrm{~mm}$.

a density of $1060 \mathrm{~kg} / \mathrm{m}^{3}$. A peak velocity of $3 \mathrm{~cm} / \mathrm{s}$ was scaled based on velocity that was previously reported [96].

\subsubsection{Simplified elliptical shaped aneurysm simulations}

The simplified elliptical shaped aneurysm was constructed in SolidWorks. The parent vessel was approximated by a hollow cylinder of $\sim 6.7 \mathrm{~mm}$ in outer diameter with a wall thickness of $1 \mathrm{~mm}$, while the elliptical shaped aneurysm was modeled after an ellipsoid having a major axis length of $13 \mathrm{~mm}$ and minor axis length of $10 \mathrm{~mm}$. Figure 4.6a-b depicts the longitudinal view of the simplified aneurysm model. The OCT catheter was then introduced into the CFD model, as shown in Figure 4.6c-d. It was observed that a large recirculation zone or vortex had formed in the sac of the aneurysm during the diastolic phase. This hemodynamic event has been well reported in the literature and is found to be stagnant in the center leading to thrombus formation [93]; however during the systolic cycle, inflow and outflow had entered and exited the neck of the aneurysm.

The cross sectional view of this aneurysm showed higher velocities in the parent vessel than the 


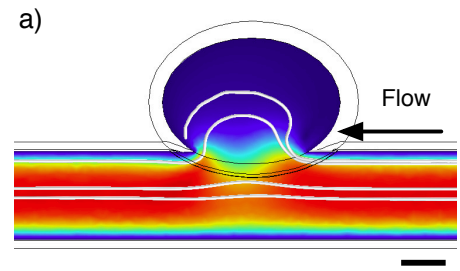

c)

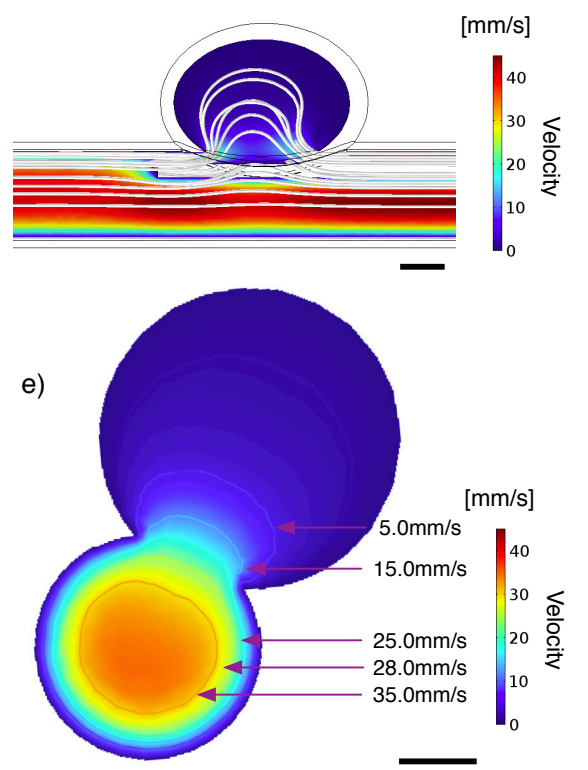

b)
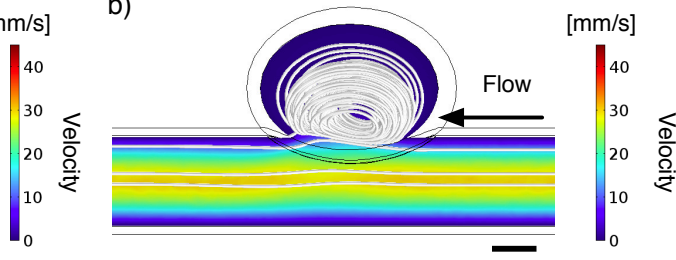

d)

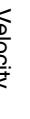

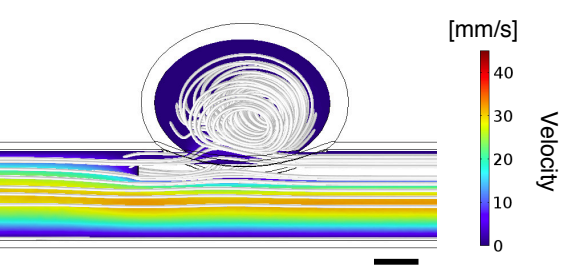

f)
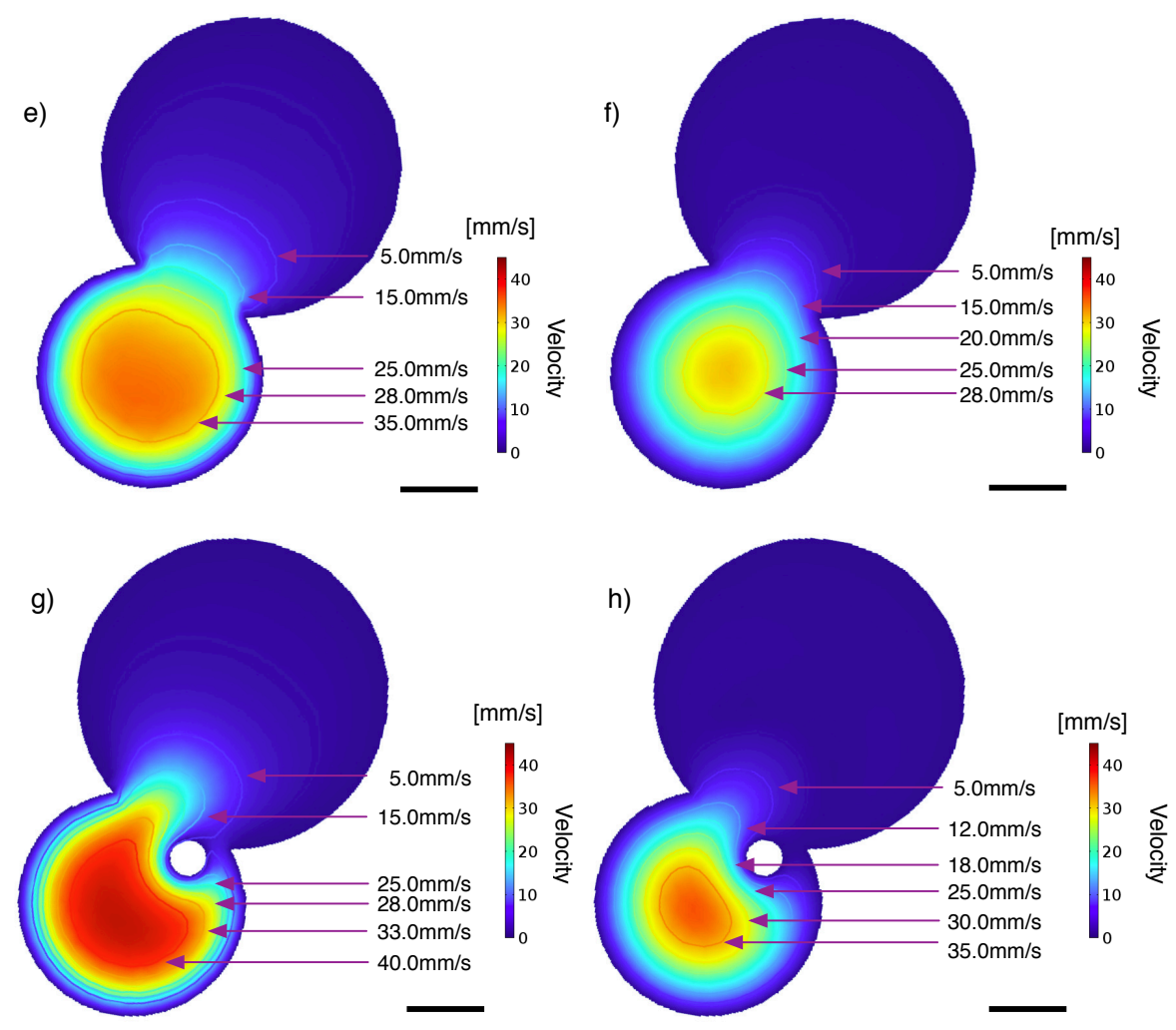

Figure 4.6: (a) Reconstructed longitudinal plane of the CFD elliptical aneurysm model during the systolic cycle. (b) Reconstructed longitudinal plane of the CFD elliptical aneurysm model during the diastolic cycle. (c) The corresponding longitudinal plane of the CFD elliptical aneurysm model during systolic cycle with the OCT catheter (d)The corresponding longitudinal plane of the CFD elliptical aneurysm model during the diastolic cycle with the OCT catheter (e) The cross sectional plane of the elliptical aneurysm model during the systolic cycle. (f) The cross sectional plane of the elliptical aneurysm model during diastolic cycle. (g) The corresponding cross sectional plane of the elliptical aneurysm model during the systolic cycle with the OCT catheter. (h)The corresponding cross sectional plane of the elliptical aneurysm model during diastolic cycle with the OCT catheter. Scale bar: $2 \mathrm{~mm}$. 
aneurysm sac. A decrease in velocity was observed at the center of the parent vessel and into the neck of the aneurysm during both the systolic and diastolic cycle (Figure 4.6(e) and Figure 4.6(f)). However, a narrowing of the flow profile had occurred adjacent to the position of the OCT catheter. Furthermore, there was an increase in velocity within the parent vessel. There were similarities between the ssDOCT images and CFD models. During the systolic cardiac cycle, low velocities were present at the neck of the aneurysm. This agreed well with the ssDOCT as lower velocity estimates were resolved at the neck of the aneurysm. This may be the result of the inflow and outflow pattern observed in the CFD. In the parent vessel, velocities were high resulting in severe aliasing in the ssDOCT image. As the velocity decreased during the diastolic cycle, resolvable velocities were seen. Overall, higher velocities were observed in the parent vessel compared to the neck of the aneurysm with ssDOCT imaging.

A virtual stent was then placed across the neck of the ideal aneurysm model (Figure 4.7). Similar to the previous chapter, the stent was virtually generated based on the shape and dimensions of intracranial stents deployed during the treatment of aneurysms. Overall, higher velocities were observed within the stented parent vessel. There were hemodynamic changes at the neck and sac of the intracranial aneurysm after stent apposition. More specifically, the stent strut that had bridged the neck of the aneurysm created a narrow stream which had diverted flow into the sac of the aneurysm (Figure 4.7(a) and Figure 4.7 (c)). Small vortices or recirculation zones had formed proximal to the neck of the aneurysm. During the diastolic cycle, streamline overlays demonstrated a separation of flow. Vortices had increased in sized compared to the systolic cycle, while some of the flow had exited the neck of the intracranial aneurysm. This flow pattern would suggest a turbulent flow near the neck of the aneurysm with a stagnant region near the center of the aneurysm sac. It was observed that the turbulent flow was more apparent with the introduction of the OCT catheter, resulting in an asymmetric vortex. The turbulent flow as well as an asymmetric vortices could lead to further injury of the endothelium and potentially decrease the structural integrity of the aneurysm wall. In the transverse plane where a vortex had formed, the sac of the intracranial aneurysm exhibited similar flow contours as the flow profile prior to the placement of the stent. By decreasing the maximum velocity range of the CFD model, it simulated the aliasing of high velocity observed in the ssDOCT image. The velocity contours from the CFD model had demonstrated similarities to the ssDOCT phase contours acquired just after peak systolic velocity. Several contours had formed in both CFD and ssDOCT images. Furthermore, an isolated contour had presented within the aneurysm. This separation in flow contours may be indicative of the deviation in phase contours observed in Figure 4.8(a), which could suggest the presence of a vortex within the sac of the aneurysm.

\subsubsection{Patient specific aneurysm simulations}

Figure 4.9 shows the result of the pre-operative CFD simulation. A relatively high velocity slipstream had appeared to flow into the sac and curved out into the connecting blood vessel. This was similar to the simplified elliptical aneurysm which had flowed through the neck of the aneurysm. A vortex or recirculation zone had formed near the entrance of the aneurysm during the diastolic cycle (Figure 4.9(b)). It was also observed that the direction of the inflow stream had diverted closer to the center of the aneurysm wall. The cross sectional view of the CFD simulation also demonstrated higher flow 
a)

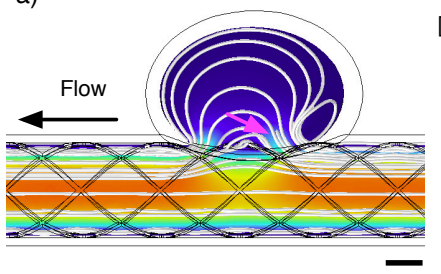

b)

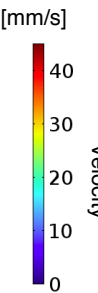

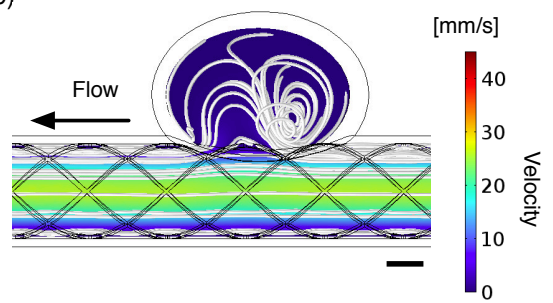

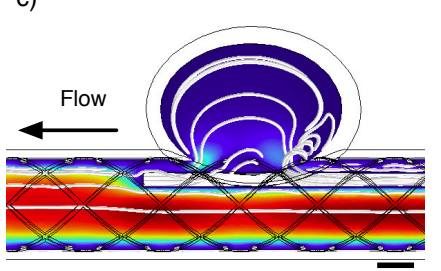

e)

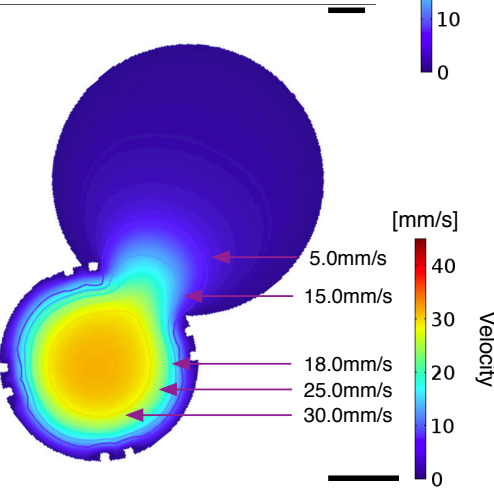

g)

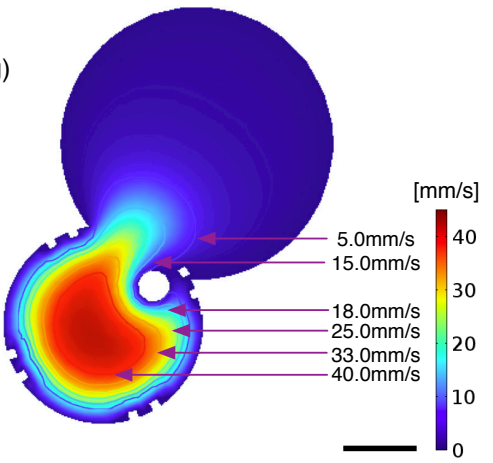

d)
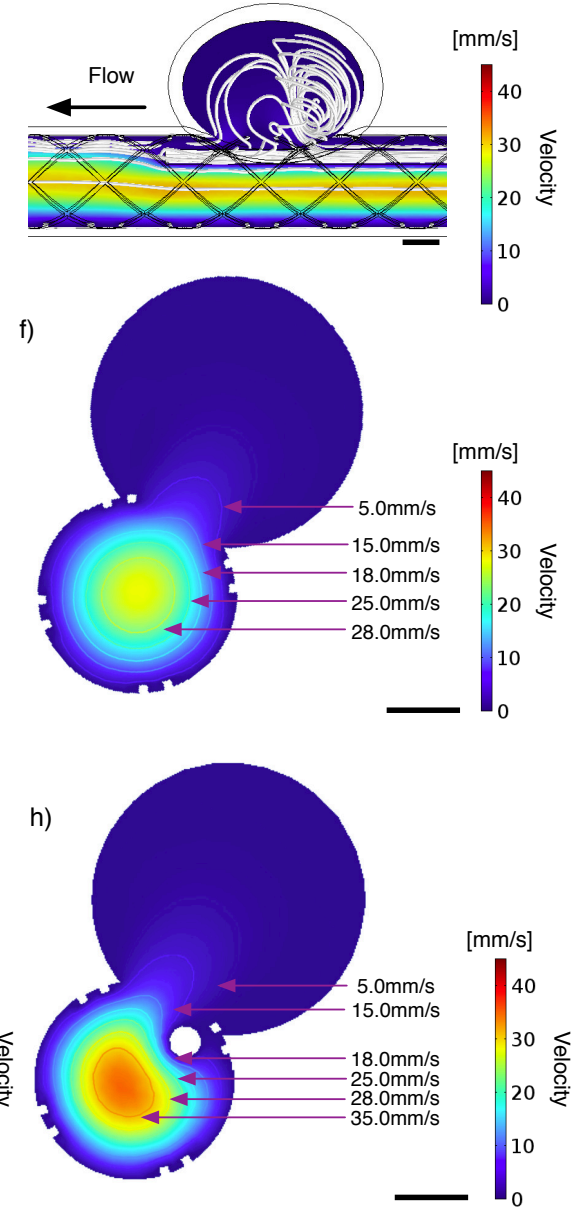

Figure 4.7: (a) Reconstructed longitudinal plane of the CFD elliptical aneurysm model after virtual stent deployment during the systolic flow. An inflow stream had formed due to the stent strut which diverted flow into the aneurysm sac (arrow). (b) The CFD elliptical aneurysm model after virtual stent deployment during the diastolic flow. (c) The corresponding systolic CFD model with the OCT catheter. (d) The resulting CFD model during diastolic flow with the OCT catheter. (e) The corresponding CFD elliptical aneurysm model after virtual stent deployment during the systolic flow in the transverse plane. (f) The corresponding the CFD elliptical aneurysm model after virtual stent deployment during the diastolic flow in the transverse plane. (g) The CFD model after the introduction of the OCT catheter during systolic flow. (h) The corresponding CFD model with the OCT catheter during diastolic cycle. Scale bar: $2 \mathrm{~mm}$. 
a)

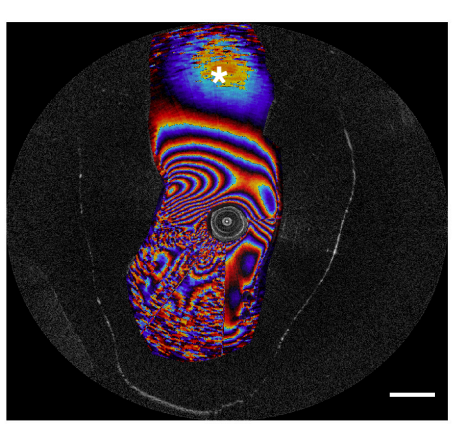

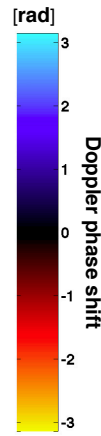

b)

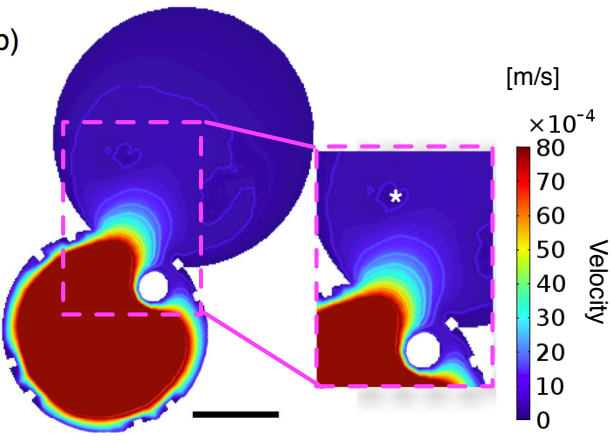

Figure 4.8: (a) The ssDOCT image within an elliptical aneurysm model acquired just after peak velocity. A deviation in phase contours $\left({ }^{*}\right)$ was observed with respect to the contours within the neck of the aneurysm. Scale bar: $1 \mathrm{~mm}$. (b) The corresponding CFD elliptical aneurysm model at the location of a vortex. The velocity dynamic range was decreased to simulate phase aliasing, which results in a separation of contours $(*)$. Scale bar: $2 \mathrm{~mm}$.

through the intracranial aneurysm sac. This was well correlated with the ssDOCT images as the systolic frame had showed high velocity (higher number of contours) in the sac of the aneurysm. In the previous section, the presence of separated contours could be the result of a vortex or recirculation zone. This could be further supported in the diastolic ssDOCT image (Figure 4.5(d)) as the two separate regions demonstrated opposing flow direction. The ability to assess the vortex pattern is advantageous as vortices exhibit a stagnant region during the systolic-diastolic cycle. Alternatively, asymmetric vortices could result in flow which impinges the aneurysm wall resulting in a high pressure region [97].

\subsubsection{Histological findings}

The histological evaluation of the PCA aneurysm is shown in Figure 4.10. Within the sac of the aneurysm, the fibrous wall contained layered thrombus composed primarily of red blood cells, fibrin, and a sparse collection of leukocytes. Thrombus that were located at the neck of the aneurysm had demonstrated focal peripheral organization. It was also observed that blood had been extravasated from a section of damaged aneurysm wall into the adjacent cerebral tissue. It is unknown if the extravasated region is due to the change in inflow stream, which could have impinged the aneurysm wall.

\subsection{Study limitations}

The predominant limitation of this work revolved around the modeling of aneurysms. Differences between the flow phantom and CFD were demonstrated. The lumen walls of the CFD were smooth and symmetric. It was seen in the structural OCT images that the lumen wall was not completely smooth and symmetrical, which resulted in disruption in the ssDOCT images near the wall of the phantom. Furthermore, the parent vessel wall was curved in the phantom model, which was not accounted for in 
a)

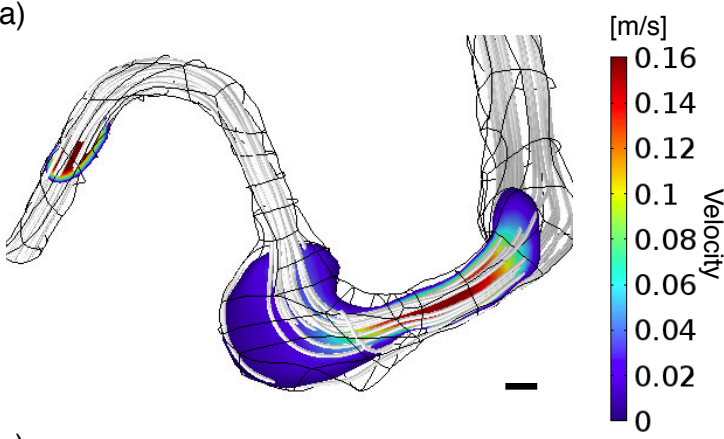

c)

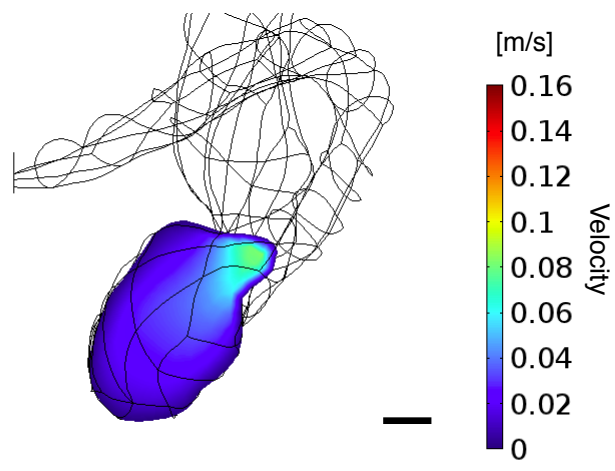

b)

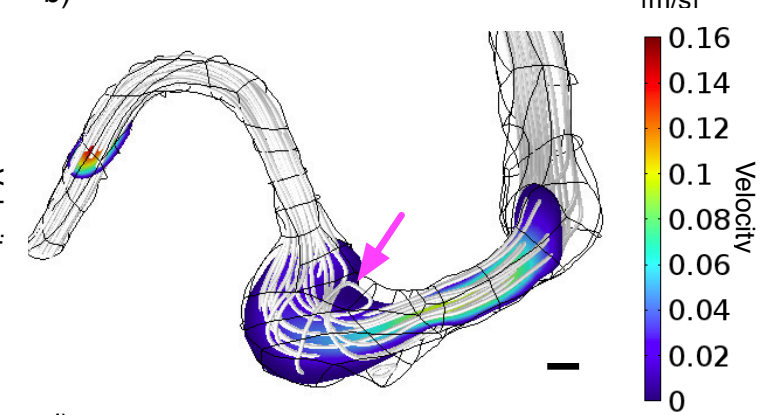

d)

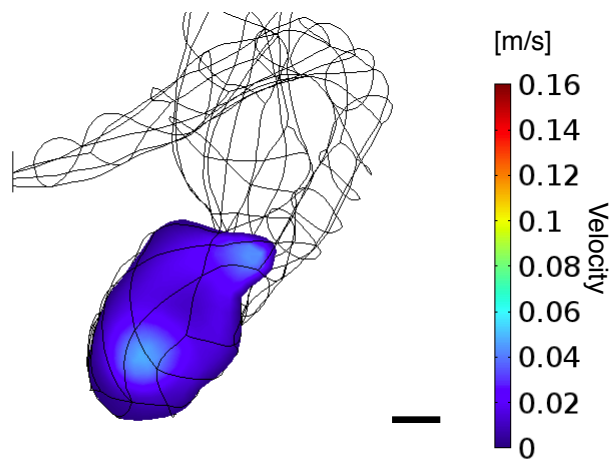

Figure 4.9: (a) Pre-operative model of a patient specific aneurysm CFD during the systolic cycle. (b) Pre-operative model of a patient specific aneurysm CFD during the diastolic cycle. The presence of a vortex was observed (arrow) during the systolic and the diastolic cycle (c) Cross sectional view of the patient specific aneurysm CFD during the systolic cycle. (d) Cross sectional view of the patient specific aneurysm CFD during diastolic cycle. Scale bar: $2 \mathrm{~mm}$.

the CFD. Finally, the injection pump of the flow phantoms did not allow for a specific cardiac profile.

The discrepancies in geometry between the CFD model and the phantom were not as pronounced as the simplified aneurysm model. Although both CFD models had indicated the presence of a vortex flow pattern within the sac of the aneurysm, the shape and speed of the vortex was different. Furthermore due to the complexity of the patient specific model, a virtual stent could not be accounted for during the simulation.

Despite these limitations, the utilization of both CFD modeling and ssDOCT imaging can provide further understanding of the local hemodynamics within the lumen of the phantom. This assessment can potentially identify significant factors (e.g. vortices, stagnant regions), that would benefit a surgeon during treatment. However, the use of this work in the clinical setting is left for future advancement, which is outlined in detail in the next chapter. 


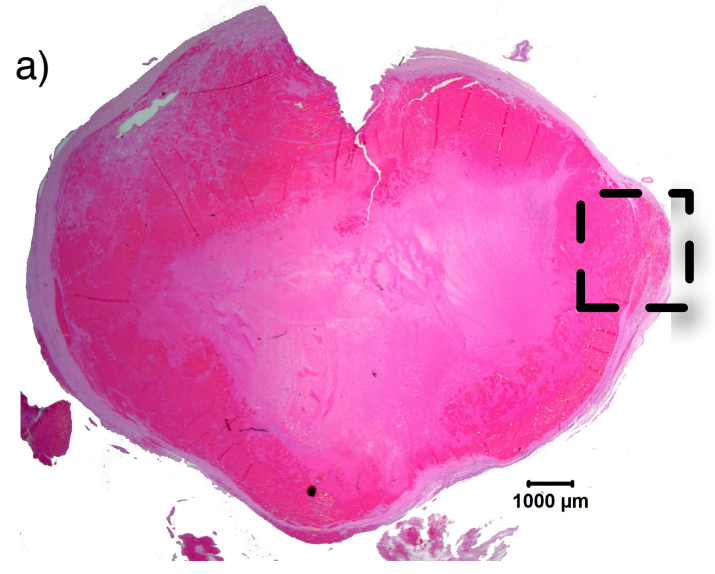

b)
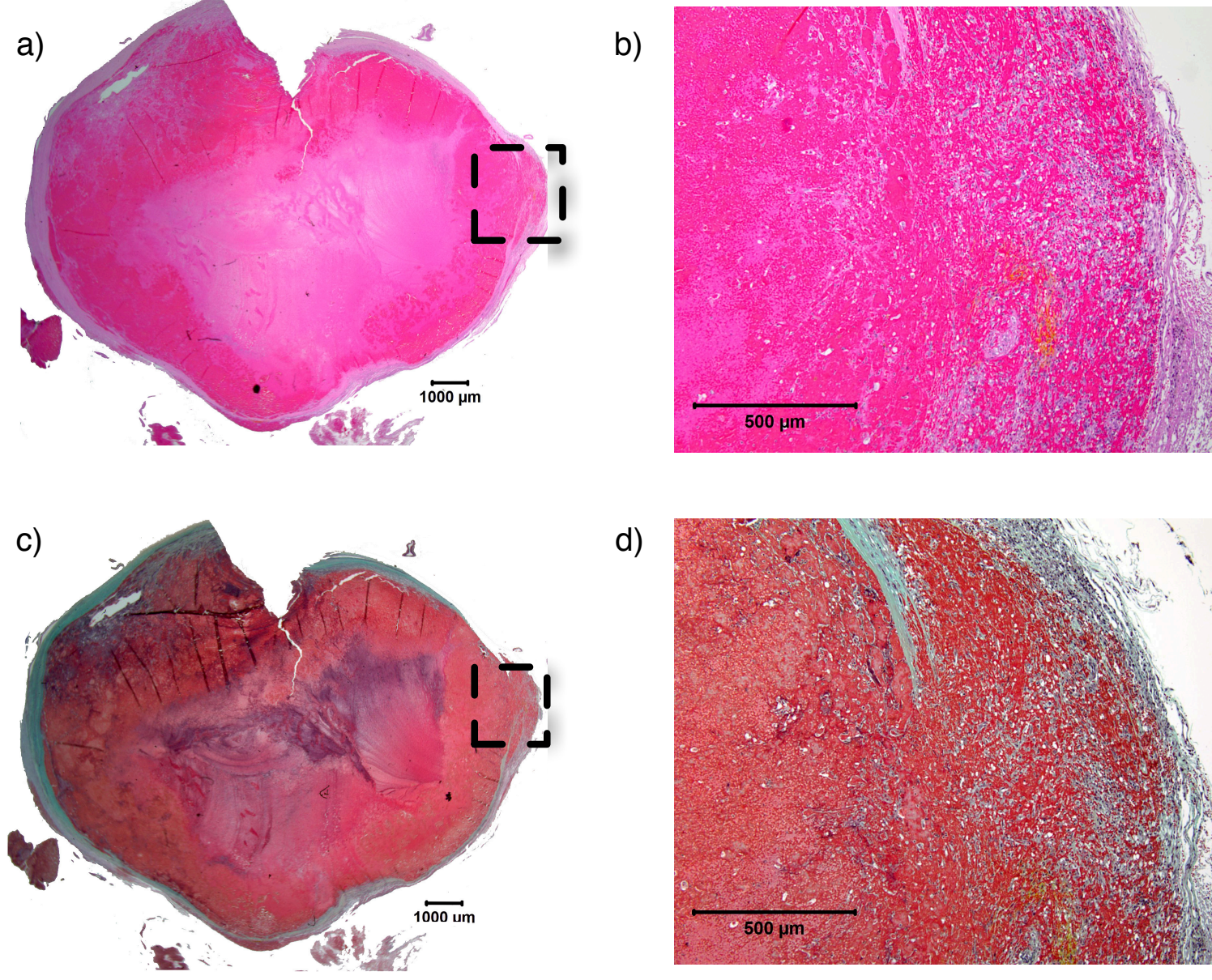

d)

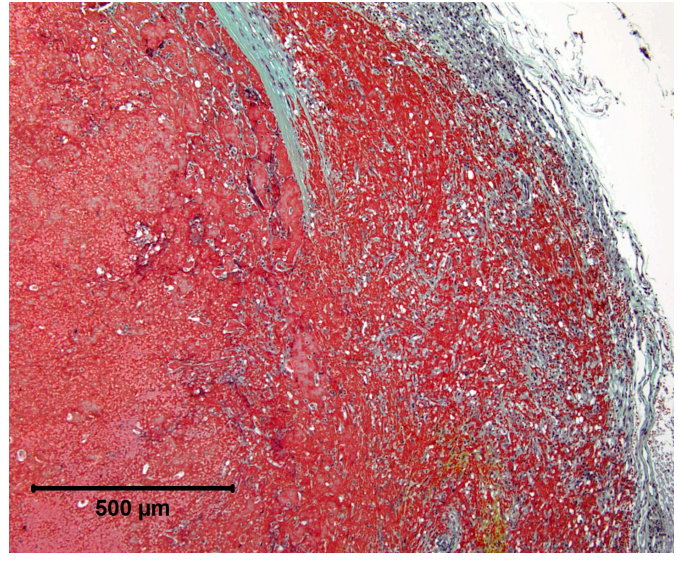

Figure 4.10: (a) Histologic sections of aneurysm stained with H\&E. Boxed regions show site of ruptured wall with extravasated blood. (b) The magnification of boxed region. (c) Corresponding histologic section stained with Movat pentachrome. Movat is a stain that highlights connective tissue. (d) Movat stain with 10x magnification of boxed area. 


\section{Chapter 5}

\section{Ex-vivo imaging of intracranial aneurysms using optical coherence tomography}

The following chapter describes ex vivo OCT imaging of human intracranial aneurysms. As observed in the previous chapters, flow dynamics can influence the arterial wall changes; however, the understanding of the clinical utility of OCT imaging to evaluate the morphological changes is limited. Furthermore, due to the size, sensitivity and limited availability of intracranial aneurysm specimens, several challenges would arise during OCT imaging and correlation with histology. We present in this chapter a tissue handling protocol for accurate correlation between histology and OCT. Several key morphological identifiers are presented that were only prominent in ruptured aneurysm specimens. However, these key identifiers observed in OCT images can be difficult to classify without histological correlations or a trained OCT reader. As a result, an optical attenuation based imaging technique will be described to assist in the correlation of histology for clinicians. The initial study was conducted on a medulloblastoma mouse model, though several intracranial aneurysm specimens were evaluated using the optical attenuation based method and are presented in this chapter. The features detected in both structural OCT and optical attenuation based OCT images may provide a priori knowledge of the neurovascular hemodynamic landscape.

The results were presented in two peer reviewed conference proceedings and a submission in the Journal of Biomedical Optics. A brief summery of the study will be presented in the following section.

1. Vuong, B., Skowron, P., Kiehl, TR., Kyan, M., Garzia, L., Sun, C., Talyor, M.D., Yang, Y.X.D. (2014) Measuring the optical characteristics of medulloblastoma using optical coherence tomography. Biomedical Optics Express. 5:4405-4416 (PhD Work).

2. Vuong, B., Sun, C., Kiehl, T., Gardecki, J.A., Standish, B.A., da Costa, L., de Morais, J.V., Tearney, G.K., Yang, V.X.D. (2012) Feasibility and methodology of optical coherence tomography 
imaging of human intracranial aneurysm: ex vivo pilot study. Proceedings of the SPIE Photonics West. 82074M-82074M-8 (PhD Work).

3. Vuong, B., Standish, B., Sun, C., Tearney, G., Kiehl, R., van der Kwast, T., Koo, J., Wilson, B.C., de Morais, J., da Costa, L., and Yang V.X.D. (2011) 3D Optical Coherence Tomography and Digital Pathology. Conference on Lasers and Electro-Optics/Pacific Rim. C353 (PhD Work).

\subsection{The natural history of intracranial aneurysms}

The mechanism for the formation and rupture of intracranial aneurysms is still unknown. There has been extensive research in the pathological genesis of intracranial aneurysms which may have identified early indicators of structural failure of the blood vessels involved. Initial understanding of the formation and growth of intracranial aneurysms was related to a thinning of the vascular wall due to high hemodynamic stress [9]. More recent studies have suggested that a morphological or remodeling of vessel wall occurs prior to rupture [98-100]. Under the normal environment, endothelial and smooth muscle cells located within the arterial wall produce extracellular matrix proteins to maintain the structural integrity of the tissue under hemodynamic pressure. Upon an increase in hemodynamic stress on the arterial wall, damage to the vascular endothelium can occur. A natural compensating mechanism is triggered, causing an inflammatory response including increased luminal migration of smooth muscle cells. This mechanism has also been associated with the development of atherosclerotic plaques leading to the infiltration of white blood cells such as macrophages and leukocytes. These cells secrete enzymes that degrade the extracellular matrix protein, and thus are considered the leading factor in the rupture of aneurysms [99-101].

As previously mentioned, current clinical imaging modalities lack the high resolution required to visualize these morphological changes. OCT has the potential capability to identify the morphology of the aneurysm wall at near histological resolution in real-time. Previous studies have demonstrated visualization of the healing process in both post-endovascular coiling in ex vivo canine models and in vivo human case reports. Various indicators associated with vessel wall healing have been observed, such as the presence of endothelialization and thrombogenesis. This chapter presents initial data and identifiable features acquired through OCT imaging that are associated with histological differences between ruptured and unruptured aneurysms. To the best of our knowledge, this is the largest human ex vivo study with intracranial aneurysms using OCT.

\subsection{Ex vivo structural optical coherence tomography imaging}

\subsubsection{Human specimens}

In this study, 21 patients had undergone surgical clipping resulting in 28 distal sac resections. At the time of surgical intervention, patient information such as age, medical history, and location of the aneurysm 
Table 5.1: Summary of histological analysis

\begin{tabular}{|c|c|c|}
\hline Variable & Unruptured $(\mathbf{n}=\mathbf{6})$ & Ruptured (n = 15) \\
\hline Age Range & $37-65$ (Average: 55) & 35 -76 (Average: 52) \\
\hline Gender & 1 Female/ 5 Males & 8 Females / 7 Males \\
\hline Previous SAH (remote history) & 0 & 2 \\
\hline Hypertension & 3 & 9 \\
\hline Smoking & 2 & 1 \\
\hline Location & & 3 \\
\hline \hline Middle Cerebral Artery & 3 & 2 \\
\hline Anterior Communicating Artery & 2 & 0 \\
\hline Opthalmic & 0 & 5 \\
\hline Pericallosal & 1 & 11 \\
\hline Posterior Communicating Artery & 0 & 3 \\
\hline Posterior Inferior Cerebellar Artery & 0 & 11 \\
\hline \hline Histology findings & & 3 \\
\hline Fibrosis & 3 & 8 \\
\hline Calcifications & 3 & \\
\hline Hemosiderin & 1 & 1 \\
\hline Fresh Thrombosis & 4 & \\
\hline Organized Thrombosis & & \\
\hline
\end{tabular}

was collected. This information is presented in Table 5.1. All patients gave written informed consent, in accordance with the local ethics committees.

The resected aneurysm specimens were immediately placed in a tissue fixative solution (10\% Formalin) for two months. Due to the small size (2 to $5 \mathrm{~mm}$ ), specimens were mounted on paper using a low melting point 2\% Agarose gel (A9414, Sigma-Aldrich, USA) prior to OCT imaging. Fiducial markers were cut into the agarose in order to maintain the shape and orientation of the tissue [6,102]. In addition, arrows were marked on the bottom of the paper to signify the scan direction during 3D imaging. The development of this protocol persevered the registration landmarkers during histological processing and is outlined in Appendix 3.

\subsubsection{Optical coherence tomography imaging}

OCT imaging was performed in collaboration with Dr. Guillermo Tearney and Dr. Brett Bouma at Harvard Medical School in the Wellmen Center for Photomedicine (Boston, USA). This OCT imaging system (commonly referred to as optical frequency domain imaging (OFDI)) used in this study has been previously described [103]. Briefly, the imaging system is a polygon-based swept source laser with a center 
wavelength of $1310 \mathrm{~nm}$, a bandwidth of $141 \mathrm{~nm}$ and a scanning A-line rate of $41 \mathrm{kHz}$. The galvanometers on the bench top OCT system was set to a $5 \mathrm{~mm}$ x $5 \mathrm{~mm}$ interrogation region for all specimens. Digital sectioning on the volumetric OCT data was performed using OsiriX (Pixmeo, Switzerland) and used to correlate OCT images with histology.
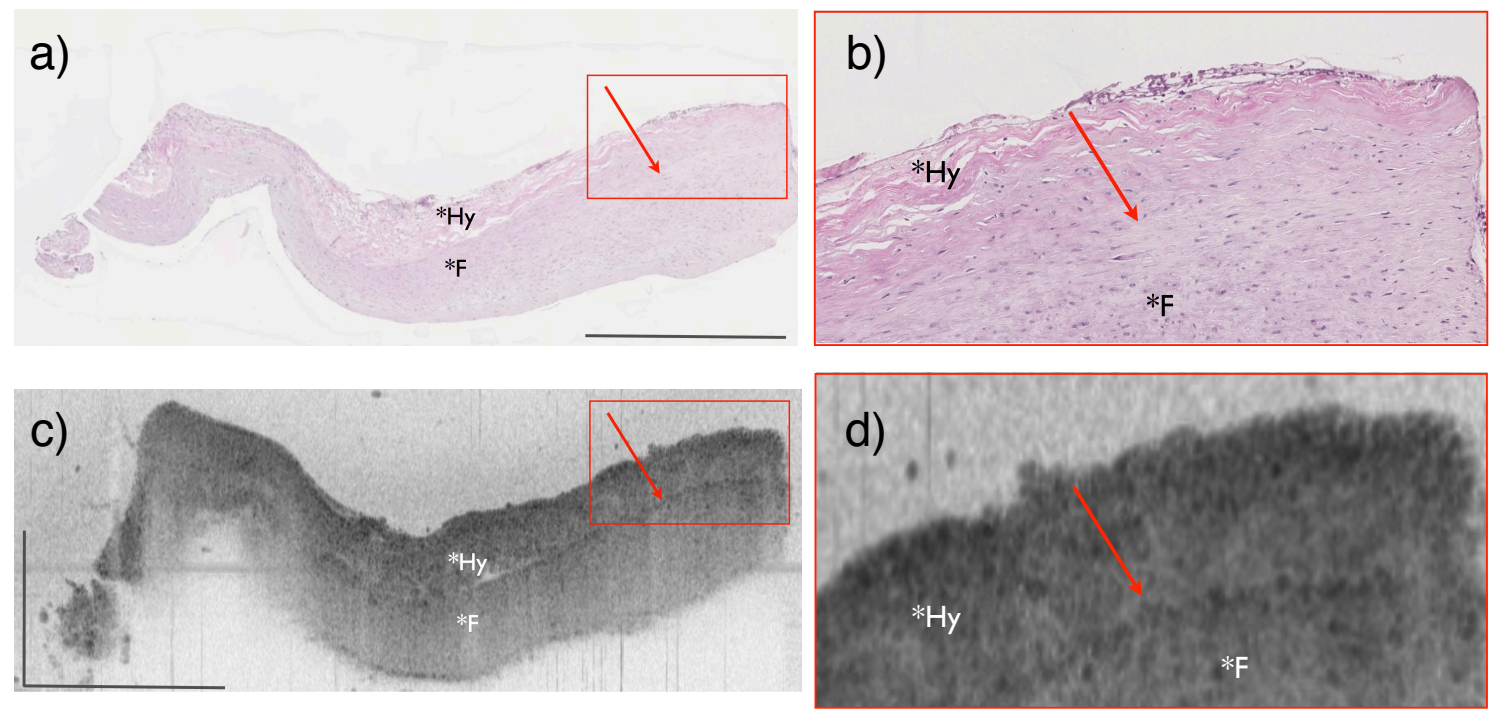

Figure 5.1: (a) $5 \mu \mathrm{m}$ section of a ruptured cerebral aneurysm sample from the ophthalmic artery $(\mathrm{H} \& \mathrm{E})$. Dense Fibrotic $\left({ }^{*} \mathrm{~F}\right)$ and hyaline $\left({ }^{*} \mathrm{Hy}\right)$ regions were observed throughout the specimen. (b) $10 \mathrm{x}$ magnification of the thin hyalinized wall and fibrotic $\left({ }^{*} \mathrm{~F}\right)$ regions. (c) There is a strong delineation between the thin wall and the collagenous layer in the OCT image. (d) Corresponding OCT magnification. Delineation between the Dense Fibrotic $\left({ }^{*} \mathrm{~F}\right)$ and thin hyalinized wall $\left({ }^{*} \mathrm{Hy}\right)$ was observed. Scale bar is $1 \mathrm{~mm}$.

\subsubsection{Correlation between optical coherence tomography imaging and his- tology}

The gross orientation of the tissue was successfully matched with OCT images. Key histological features were identified in 8 of the 28 specimens. In the healthy arterial wall, there are four distinct layers: intima, internal elastic lamina, media and adventitia layers. Remodeling of the arterial walls commonly results in a composition of intima, fibro-hyaline tissue, and adventitia. This may be due to perforation of the arterial wall, which results in the disposition of fibrin, preceded by aggregation of blood and intramural thrombosis [100]. Fibroblast infiltration occurs in chronic aggregation of blood cells [100]. As a result, fibroblast infiltration may be seen as a compensatory mechanism to maintain structural integrity, as it is common during wound healing. In addition, the presence of hyalinized tissue has been connected to mild to moderate hypertension [104]. The differentiation between the cellular fibrotic tissue and paucicellular hyaline (Hy) tissue were seen in a ruptured aneurysms specimen in OCT. Specifically, the separation between the densely cellular fibrotic (F) tissue and hyaline tissue were seen in Figure 5.1. The corresponding histological analysis showed fibroblast infiltration in the densely cellular fibrotic tissue 
and few infiltrations in the hyaline tissue. These identifiable features were also observed in pathological studies of unruptured and ruptured aneurysm [101]. Kataoka et al. [100] reported the presence of thick intima-like walls in unruptured aneurysms, whereas ruptured aneurysm exhibit thin hyalinized walls.

Figure 5.2 shows an unruptured aneurysm specimen that consists of fibrous tissue (F) and hemosiderin granules $(\mathrm{H})$ near the adventitial side and micro-calcification. Hemosiderin granules were visualized as highly reflective elements in the corresponding OCT image (Figure 5.2(b)). The majority of hemosiderin laden regions are the resultant form of macrophages within organized thrombosis. Generally, prior hemorrhage can lead to the accumulation of macrophages and take up hemosiderin. Minor hemorrhages can often proceed to a major rupture within hours, days or weeks. Fibrous tissue could be observed above the hemosiderin deposit. Volumetric rendering of the specimens provided visualization of large calcium deposits (Ca) and clusters of hemosiderin near the adventitia. The presence of large calcium deposits is a key identifier of the vessels' compensatory mechanism, as they are believed to increase the structural integrity of the vessel wall.

An inflammatory response leading to the formation of atherosclerotic calcification was observed in this study. OCT demonstrated clear delineation of large calcifications from fibrotic tissue in unruptured aneurysm specimens. However, micro-calcifications were predominantly found in ruptured specimens and could not be identified with OCT. This would suggest that large atherosclerotic calcification provides structural support in the aneurysm wall.

One of the major technical limitations of this study was the handling of the aneurysm specimen. It was found that a number of specimens either suffered from tissue folding, inability to identify the lumen wall during imaging, and presence of excess surface blood. Improvement of the transition from resection to tissue embedding is required, as well as methods to reduce excess blood are required prior to imaging. Furthermore, the long fixation period would degrade the OCT image quality. Unfortunately, ex-vivo aneurysm specimens are rare as standard of care for the treatment of aneurysm is endovascular coiling or clipping without resection.

\subsection{Ex vivo optical coherence tomography - optical attenuation imaging}

In the previous section, the differentiation of key microstructurual features can assist in the risk assessment of an unruptured aneurysm. However to identify these key morphological features requires a trained OCT reader. Due the near histological resolution of OCT, optical attenuation coefficient can be utilized as a mechanism to visualize OCT as a pseudo histology image. This was initially employed on genetically modified mice with medulloblastoma. Preliminary images of several intracranial aneurysms were also assessed using the optical attenuation imaging technique.

Medulloblastoma is among the most prominent malignant pediatric tumors and has the greatest tendency to metastasize [105]. Generally, this tumor infiltrates the cerebellum and can cause occlusion of cerebrospinal fluid, which can result in hydrocephalus [106]. The current standard of care is to treat this tumor with a combination of surgical resection, high-dose radiation, and chemotherapy. However, 

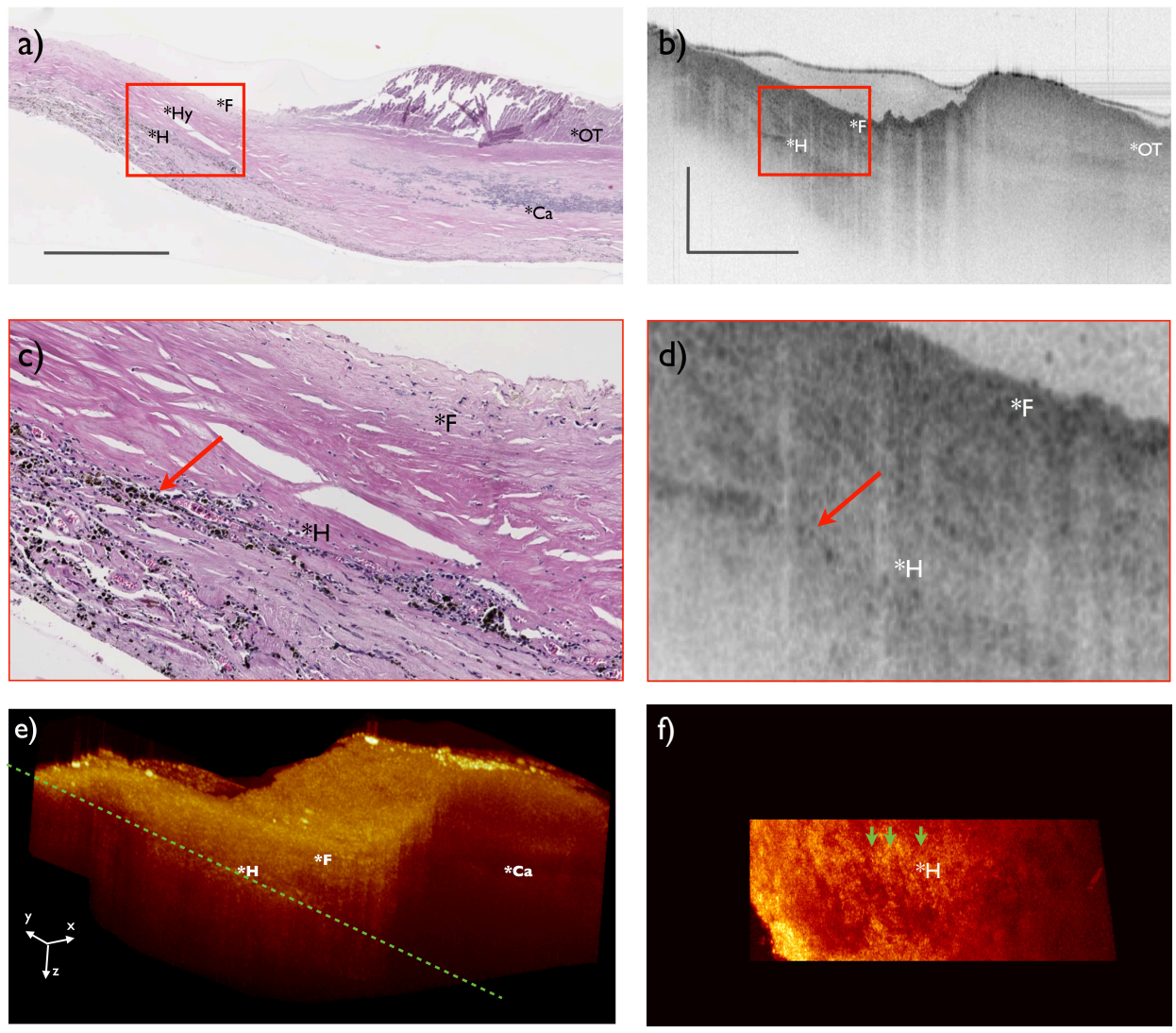

Figure 5.2: (a) $5 \mu \mathrm{m}$ section of an unruptured cerebral aneurysm sample from the pericallosal artery, stained with $\mathrm{H} \& \mathrm{E}$. Various stages of fibrosis, such as regions of fibrotic tissue $\left({ }^{*} \mathrm{~F}\right)$ was observed throughout the sample. The presence of calcium $\left({ }^{*} \mathrm{Ca}\right)$ and dense hemosiderin $\left({ }^{*} \mathrm{H}\right)$ deposits suggests a healing process of the vessel. Hemosiderin is commonly found in vessels that have experienced prior hemorrhages. The deep purple area observed is the lumen of an organized thrombus $\left({ }^{*} \mathrm{OT}\right.$; clear material on luminal surface is $2 \%$ agarose gel, related to OCT analysis). (b) OCT rendition of the aneurysm specimen. The hemosiderin $\left({ }^{*} \mathrm{H}\right)$ is clearly visible in the optical image. Additionally, the boundary between collagenous tissue and hemosiderin ${ }^{*} \mathrm{H}$ ) can be resolved. However, the calcium deposits could not be visualized due to attenuation of light through the degenerated tissue. (c) 10x magnification of the region with hemosiderin $\left({ }^{*} \mathrm{H}\right)$ and fibrosis $\left({ }^{*} \mathrm{~F}\right)$. (d) Corresponding OCT magnification. Alignment of hemosiderin $\left({ }^{*} \mathrm{H}\right)$ along the adventitial side was resolved. Furthermore, the delineation between the fibrotic $\left({ }^{*} \mathrm{~F}\right)$ and hyalinized regions was observed. (e) 3-dimensional OCT visualization of the intracranial aneurysm. Digital resection can be achieved (green dotted line) in the volumetric dataset. (f) This resulted in the detection of clusters of hemosiderin. Scale bar is $1 \mathrm{~mm}$. 
radiation has shown to cause significant impairment of intelligence and neurocognitive dysfunction [107]. Statically, the completeness of surgical resection is directly correlated to the child's quality of life [108]. Unfortunately, during surgical resection, the optical contrast between normal brain and tumor tissue can be difficult to distinguish without additional tools.

\subsubsection{Medulloblastoma animal model}

In this study, 5 genetically modified mouse model (MATH1-GFP;Ptch+/- ) were utilized. These mouse models expressed a green fluorescent protein (GFP) reporter, which was restricted to medulloblastoma tumor tissue that had developed in the cerebellum [1]. Normal mouse model $(\mathrm{N}=2)$ were also employed as a control. In both tissue types, the entire mouse brain was harvested and placed on ice in artificial cerebrospinal fluid prior to imaging. Fluorescent images of the models were taken using a Olympus SzX16 microscope that consisted of a $395 \mathrm{~nm}$ excitation laser. This protocol was approved by the Animal Care Committee at The Toronto Centre for Phenogenomics (Toronto, Canada).

\subsubsection{Optical coherence tomography - optical attenuation imaging}

The harvested brains were imaged using a laboratory built polygon based swept source laser, which had a full width half maximum bandwidth $110 \mathrm{~nm}$ centered at $1310 \mathrm{~nm}$ at an A-line frequency of $36 \mathrm{kHz}$. A fiber-based interferometer was utilized to combine the light from the reference and sample arm, which was was detected by a balanced photodetector (PDB-440 Thorlabs, USA).

The OCT optical attenuation (OCT-OA) imaging has been previously demonstrated in ex vivo and in vivo images of human glioma specimens [109-111], atherosclerosic plaques [112-115], human axillary lymph [116], and other various tissues types [117-119]. The basis of the OCT-OA algorithm consists of a model that accounts for the depth roll-off of the swept source laser and the focusing optics in the sample arm. Assuming that only single scattering events were measured, the measured OCT can be approximated by

$$
<I_{d}(z)>=I_{o} T(z) S(z) \exp \left[-\mu_{t} z\right]
$$

where the measured OCT signals, $I_{d}(z)$, is averaged over several realizations, denoted by $<>$. Averaging improved the signal-to-noise ratio and removed speckle generated by the tissue. In this study, 4 consecutive frames were used. $I_{o}$ is the locally available intensity and $\mu_{t}$ is the optical attenuation coefficient. The longitudinal point spread function of the OCT system is denoted as $T(z)$ and is characterized by $[113]$

$$
T(z)=\left[\left(\frac{z-z_{o}}{z_{R}}\right)^{2}+1\right]^{-\frac{1}{2}},
$$

where the position of the beam waist was $z_{o} \approx 19.4 \mu \mathrm{m}$ and the Rayleigh length was $z_{R} \approx 1 \mathrm{~mm}$. The 
signal roll-off with depth $(S(z))$ was taken into account as [113]

$$
S(z)=\exp \left[-\left(\frac{z}{z_{w}}\right)^{2}\right]
$$

where $z_{w}$ is the roll-off function and was measured to be $3 \mathrm{~mm}$ by translating a mirror in the sample arm until the amplitude had decreased to $8 \mathrm{~dB}$ [120]. Linearization of the intensity signal was achieved by logarithmic compression [113]

$$
i_{d}=\log \left[<I_{d}(z)>\right]-\log [T(z)]-\log [S(z)] .
$$

Thresholding was used to detect the surface of the tissue and a least square fit was performed on Equation 5.4 with a variable minimum window length of the following model [113],

$$
i(z)=\log \left[I_{o}\right]-\mu_{t} z .
$$

The goal of the algorithm is to linearly fit Equation 5.5 to Equation 5.4. This was achieved by a cost versus fit procedure within an isolate homogeneous region to estimate $\mu_{t}$ (the slope). In previous literature, the minimum length of the window for this fitting procedure was fixed to a set length. However in this study, an image segmentation technique was used to dynamically adjust the minimum length and initialize the boundary of the tissue layers. The image segmentation technique used was a mean shift segmentation algorithm, which is a robust feature space analysis [121]. This method operates by initially choosing a random data point. Evaluation of the mean data points of the cluster was performed within a set radius, where the center of the radius is shifted to the calculated mean point. The process is repeated until the calculated center radius converged. Mean shift segmentation was chosen because the center of the cluster region that the technique converges at is an estimate of the location of a homogenous tissue boundary. Furthermore, the estimate is performed without the user's input (unsupervised).

A calibration step was utilized in order to remove the OCT system dependencies $(S(z) \& T(z))$. A homogeneous phantom (polystyrene microspheres diluted in distilled water) with known optical properties $\left(\mu_{p}\right.$ and $\left.I_{p}\right)$ was imaged prior to imaging of the specimen [116]. This calibration process involved the modification of Equation $5.4 \& 5.5$ to account for the optical properties of polystyrene microspheres.

$$
\begin{gathered}
i_{c}=\log \left[<I_{d}(z)>\right]-\log \left[<I_{p}(z)>\right] \\
i(z)=\log \left[\frac{I_{o}}{I_{p o}}\right]-\mu_{c} z \\
\mu_{c}=\mu_{t}-\mu_{p},
\end{gathered}
$$

where $\left\langle I_{p}(z)\right\rangle$ is the measured OCT signals of the phantom, $I_{p o}$ is the local available intensity of the phantom, $\mu_{c}$ is the calibrated optical attenuation coefficient and $\mu_{p}$ is the optical attenuation coefficient of the phantom $\left(\mu_{p}=0.69 \mathrm{~mm}^{-1}\right)$, as previously reported [116]. The simplified flow diagram of the 
optical coherence tomography technique can be found in Appendix 4.

\subsubsection{Medulloblastoma and optical coherence tomography - optical attenu- ation}

OCT-OA imaging was initially applied to medulloblastoma tissues. Figure 5.3 shows the conventional OCT imaging of a normal cerebellum section in the mice model (MATH1-FGP;Ptch+/-). Normal cerebellum consists of a molecular layer, granular layer, Purkinje cell layer and white matter. It can be seen that OCT-OA could clearly distinguish the molecular layer $\left({ }^{*} \mathrm{M}\right)$, granular layer $\left({ }^{*} \mathrm{G}\right)$ and white matter $\left({ }^{*} \mathrm{~W}\right)$, which can be difficult to distinguish in conventional OCT. More specifically, the granular layer exhibited a higher optical attenuation coefficient than the molecular layer and white matter. Contralateral to this hemisphere, there was a boundary between normal cerebellum and a large tumor mass. From the gross histology of the mouse brain, the differentiation between normal cerebellum and medulloblastoma was difficult to determine (Figure 5.3(a)). Figure 5.4(b) presents an OCT image at a boundary between normal cerebellum and infiltrated medulloblastoma. Different cellular densities within the tumor were observed in the OCT-OA and confirmed by H\&E. The OCT-OA image clearly identifies the regions of medulloblastoma and normal tissue, whereas conventional OCT was not as distinct. This was due to higher optical attenuation coefficient exhibited in infiltrated medulloblastoma. Overall, the optical attenuation coefficient mean / standard deviation were noticeably different between key morphological structures and are summarized in Table 5.2. For each specimen, the tissue types from Table 5.2 were identified from both the histology and OCT-OA. A region of interest was selected and used for calculate the mean and standard deviation. A T-test was performed with respect to each tissue type and is well described in the previous literature [1].

It was observed that the high cellularity in medulloblastoma consisted of a mean optical attenuation coefficient of $>7.6 \mathrm{~mm}^{-1}$. Previous studies have reported that renal cell carcinoma was measured to have a median optical attenuation coefficient value of $8.85 \mathrm{~mm}^{-1}$ [122]. In terms of normal tissues, healthy coronary lumen consisted of an optical attenuation coefficient value between 2 to $5 \mathrm{~mm}^{-1}$ [113]. However, the optical attenuation coefficient values was not the unique quantifier. The geometric shape and location of the OCT-OA image was accounted for in order to identify the tissue type. For example, an "inverse boot-like" shape of high optical attenuation was classified as granular layer, which was verified through histology.

\subsubsection{Intracranial aneurysm and optical coherence tomography - optical at- tenuation}

Preliminary studies of OCT-OA imaging were also conducted with ex vivo human intracranial aneurysms using a Thorlabs swept source laser. Briefly, the swept source laser had a center wavelength of a 1310 $\mathrm{nm}$ with a bandwidth of $\sim 110 \mathrm{~nm}$ and an A-scan rate of $8 \mathrm{kHz}$. Figure 5.5(a) shows the histology of an unruptured aneurysm consisting of irregular fibrous thickening $\left({ }^{*} \mathrm{IF}\right)$, fibrotic tissue $\left({ }^{*} \mathrm{~F}\right)$ and hemosiderin $\left({ }^{*} \mathrm{H}\right)$. Similar to the previously presented structural OCT images, hemosiderin had exhibited 

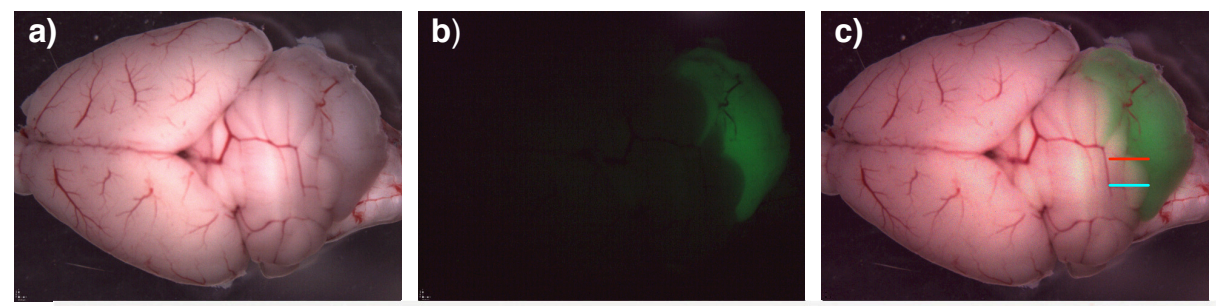

d)

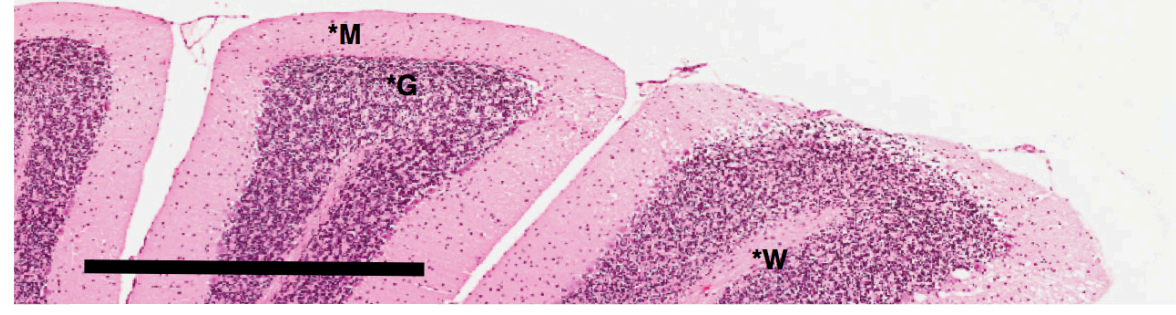

e)

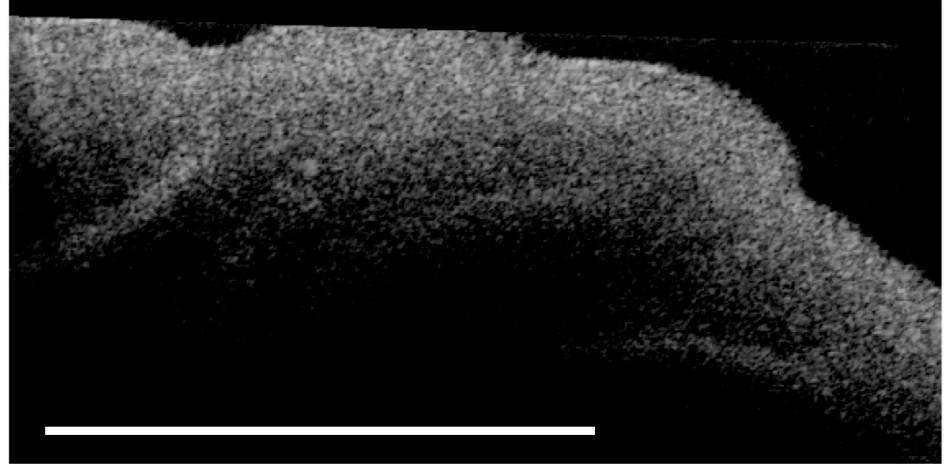

[a.u.]

f)
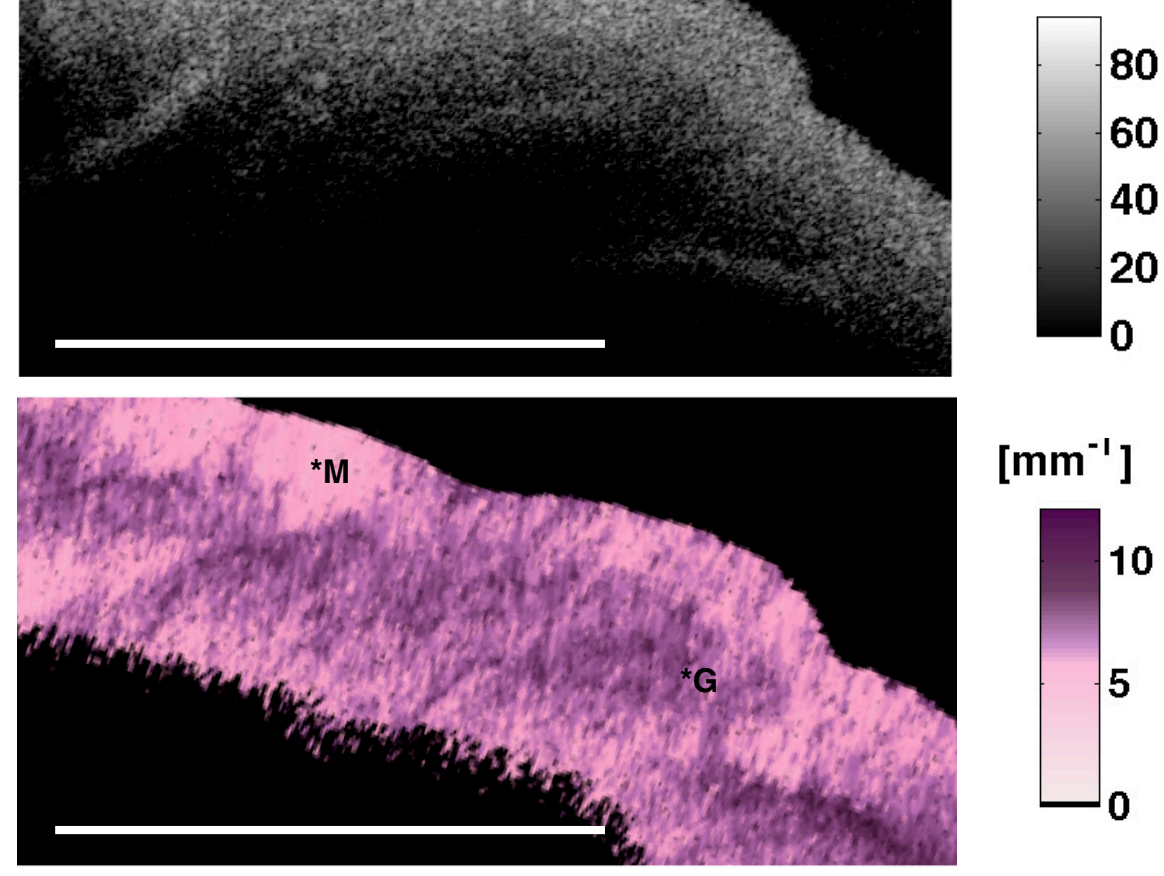

Figure 5.3: (a) Gross histology of harvested Math1-GFP;Ptch ${ }^{+/-}$mouse model. (b) The corresponding fluorescence image. (c) The combined gross and fluorescence image. Normal cerebellum (cyan line) and an interface of normal/medulloblastoma (red line) scans were taken. (d) H\&E section of the cerebellum. Grey matter consisting of molecular layer $\left({ }^{*} \mathrm{M}\right)$ and granular layer $\left({ }^{*} \mathrm{G}\right)$ was observed. Underneath the granular layer, regions of white matter are present $\left({ }^{*} \mathrm{~W}\right)$. (e) The associated structural OCT with minimal resolved features. (f) The corresponding OCT-OA image consisted of a layered structure. The shape and location of these layers are similar to the molecular layer $\left({ }^{*} \mathrm{M}\right)$ and granular layer $\left({ }^{*} \mathrm{G}\right)$. Scale bar $500 \mu \mathrm{m}$ [1]. (Reprinted with permission of the Optical Society of America) 

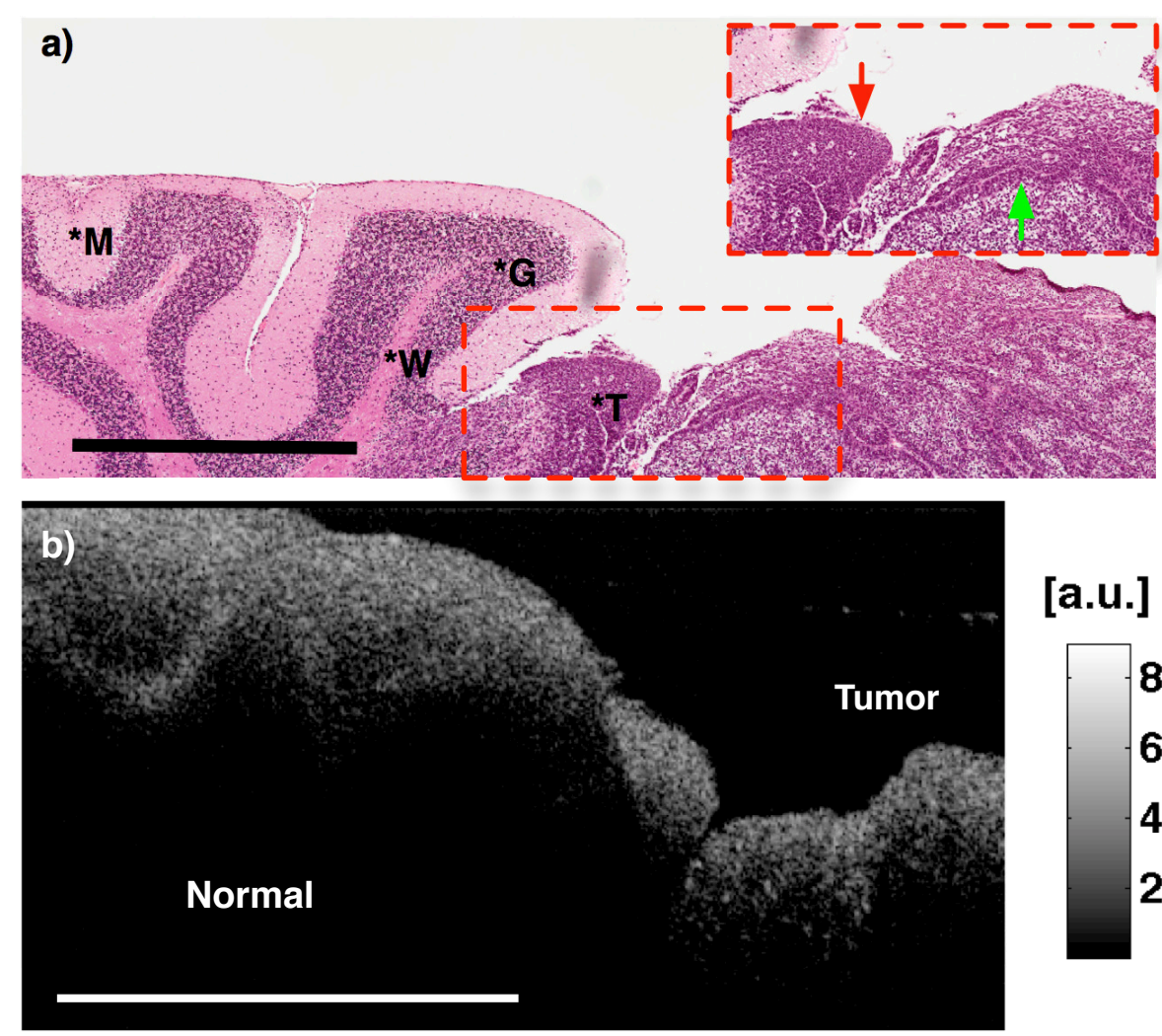

[a.u.]
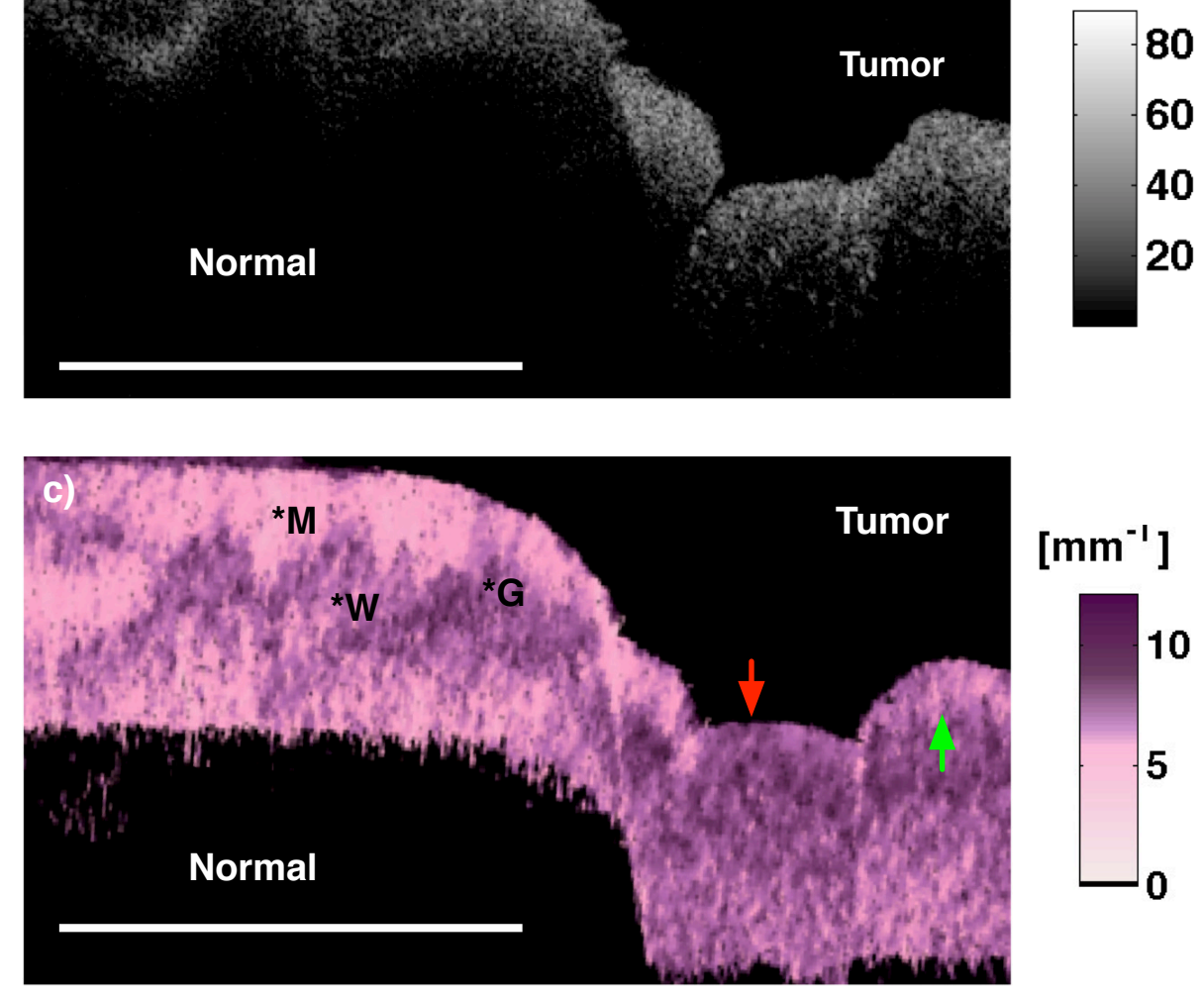

20

(1)

Figure 5.4: (a) H\&E section of the cerebellum in a Math1-GFP;Ptch ${ }^{+/-}$model. Grey matter consisting of molecular layer $\left({ }^{*} \mathrm{M}\right)$ and granular layer $\left({ }^{*} \mathrm{G}\right)$ was observed. Underneath the granular layer, regions of white matter $\left({ }^{*} \mathrm{~W}\right)$ are present. Regions of medulloblastoma resided on the right side $\left({ }^{*} \mathrm{~T}\right)$. Within the red dashed box, medulloblastoma regions that were adjacent to normal cerebellum demonstrated very dense cellular proliferation (red arrow). On the right side of the medulloblastoma region, superficial cells appear to be sparsely spaced (green arrow); however a lining of dense cells was located below. (b) The corresponding structural OCT with minimal resolved features. (c) The OCT-OA image consisted of a layered structure and erratic high attenuation region. In normal tissue the layers consisted of the molecular layer $\left({ }^{*} \mathrm{M}\right)$ and granular layer $\left({ }^{*} \mathrm{G}\right)$. The erratic high attenuation region was where medulloblastoma $\left.{ }^{*} \mathrm{~T}\right)$. Scale bar $500 \mu \mathrm{m}$ [1]. (Reprinted with permission of the Optical Society of America) 
Table 5.2: Summary of optical attenuation coefficient by tissue type [1]. (Reprinted with permission of the Optical Society of America)

\begin{tabular}{|l|l|l|}
\hline Tissue Type & $\mu_{t}\left(\mathrm{~mm}^{-1}\right)$ & $\mathrm{p}$-value \\
\hline Molecular Layer $(\mathrm{n}=3)$ & $4.8+/-0.6$ & 0.015 \\
\hline Granular Layer $(\mathrm{n}=3)$ & $7.1+/-0.3$ & 0.078 \\
\hline White Matter $(\mathrm{n}=3)$ & $5.2+/-0.4$ & 0.034 \\
\hline Medulloblastoma $(\mathrm{n}=3)$ & $7.8+/-0.4$ & 0.002 \\
\hline
\end{tabular}

a high reflectivity and was seen at the bottom of the image. However, irregular fibrous thickening on the aneurysm wall was difficult to distinguish in the structural OCT. The corresponding uncalibrated OCT-OA image demonstrated a high optical attenuation coefficient layer on the wall of the sample, which had corresponded to the irregular fibrous thickening. Several regions of fibrotic tissue (regular) were observed throughout the tissue. These regions had exhibited lower optical attenuation coefficients. Lastly, the hemosiderin that was seen in both the histology and structural OCT showed a higher optical attenuation coefficient.
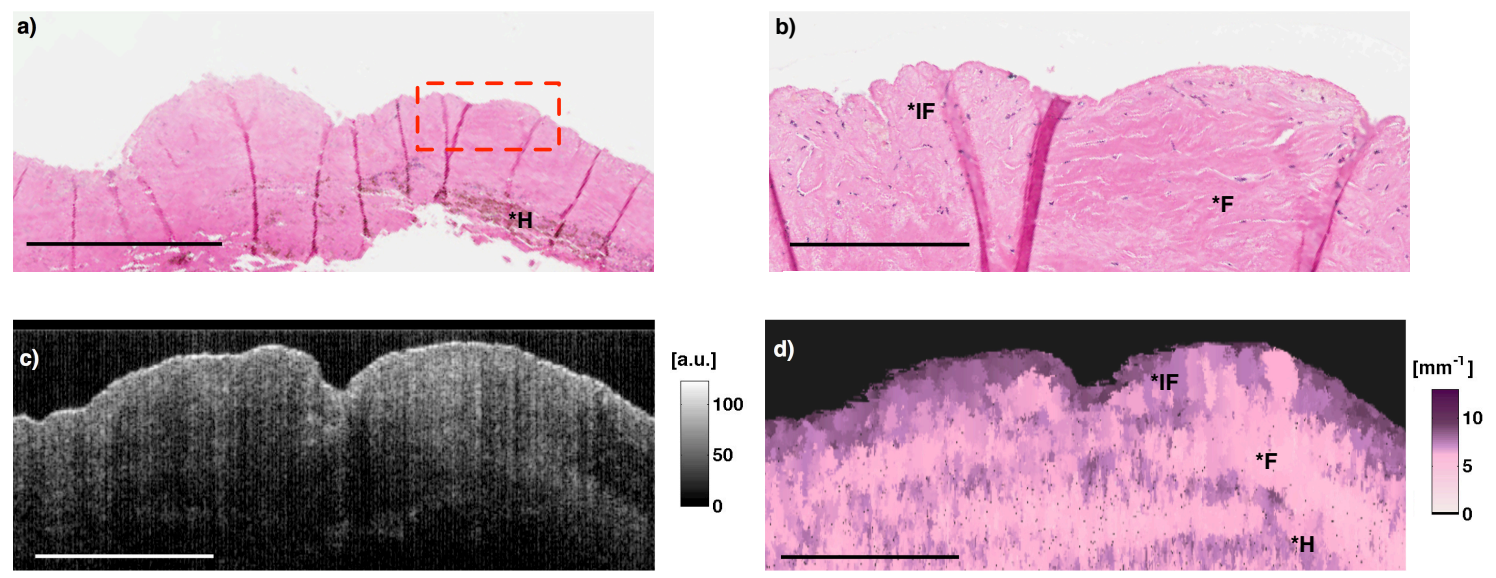

Figure 5.5: (a) H\&E stained section of an unruptured intracranial aneurysm. A large density of hemosiderin $\left({ }^{*} \mathrm{H}\right)$ was observed. Scale bar: $1 \mathrm{~mm}$ (b) Magnification of the aneurysm wall. Irregular fibrous thickening $\left({ }^{*} \mathrm{IF}\right)$ along the vessel wall was observed. Scale bar: $200 \mu \mathrm{m}$. (c) Structural OCT image of the unruptured intracranial aneurysm. (d) The corresponding uncalibrated OCT-OA image. Scale bar: $1 \mathrm{~mm}$.

A ruptured aneurysm specimen was also imaged using the uncalibrated OCT-OA technique (Figure 5.6). Histological evaluation of the tissues showed a thickening of the aneurysm wall. A layer of cellular structure was seen in between non-cellular layers. A large dense cellular layer/region was observed in the OCT-OA image and was believed to be the cellular portion of the media. Lower attenuation coefficient surrounding the high attenuation coefficient (cellular layer) was indicative of the non-cellular fibrotic tissue observed in the tissue. 

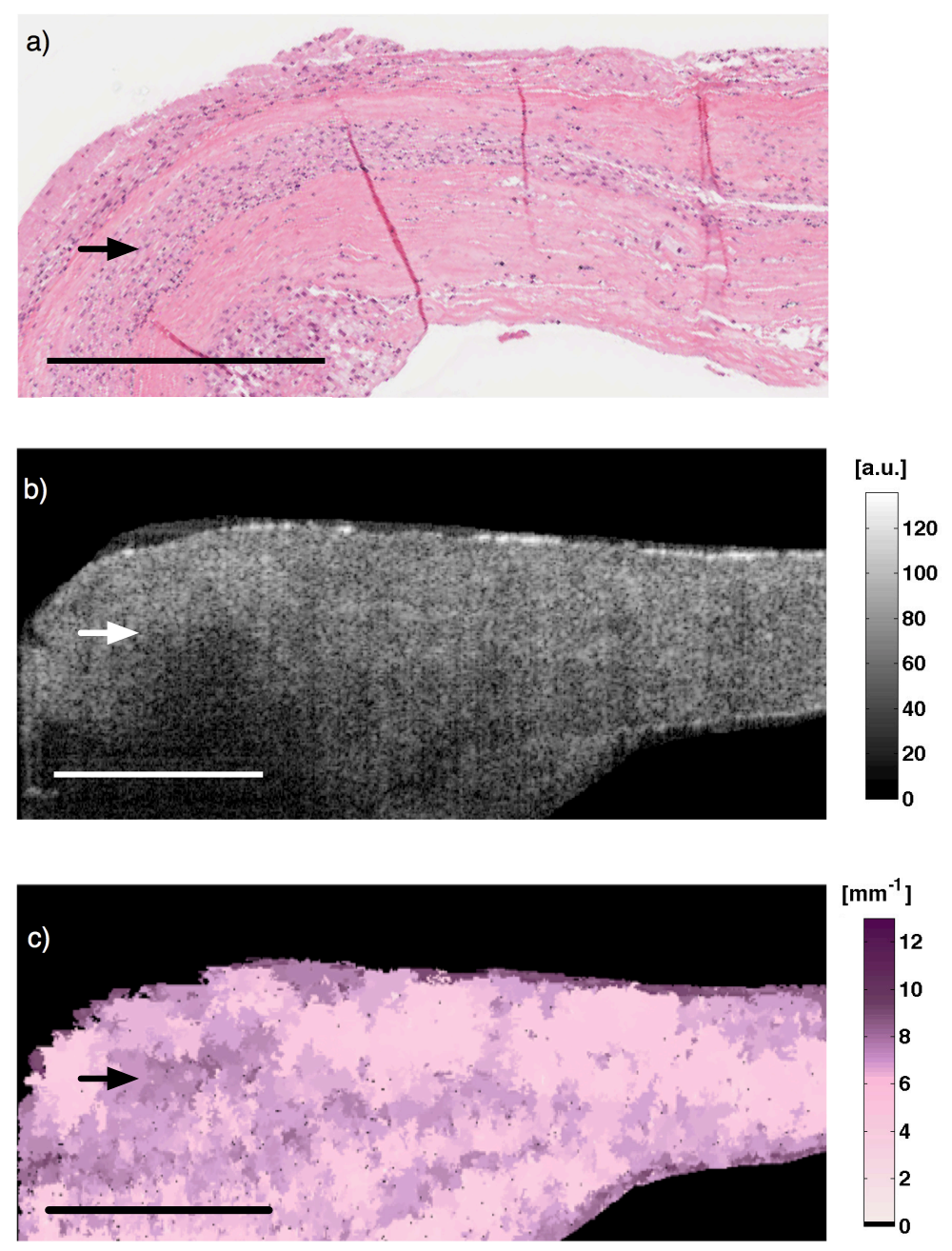

Figure 5.6: (a) H\&E stained section of a ruptured intracranial aneurysm. (b) Structural OCT image (c) The corresponding uncalibrated OCT-OA image. Scale bar: $1 \mathrm{~mm}$.

From this preliminary result, OCT-OA had provided further identification of key morphological structures in the aneurysm wall. The combination of the structural OCT imaging observed in the previous section and OCT-OA may provide additional information during the rupture risk assessment of intracranial aneurysms.

\subsubsection{Limitation of optical coherence tomography - attenuation imaging}

The predominant limitation of this, which was previously outlined by van Soest et al. [113], was the inability to measure the backscattering coefficient. This had resulted in artefacts and overestimates of optical attenuation coefficients at sharp interfaces between different tissues. Among the previously reported limitations of OCT-OA [113], the mean shift segmentation process had significantly increased 
the computation time. In order to translate the method to in vivo OCT-OA imaging, parallel computing and graphical processing units could be employed for real-time imaging. Further optimization of the radius parameter in the mean shift segmentation would also need to be explored, as well as other speckle reduction techniques [115]. Additionally, while the mean shift segmentation was initially utilized other segmentation methods should be explored. Despite these factors, the preliminary results from this study may be utilized in brain tissue and exploited in the clinical setting for surgical guidance or risk assessment. 


\section{Chapter 6}

\section{Summary, Conclusion, and Future Work}

\subsection{Concluions}

In this thesis, a novel intravascular Doppler signal processing technique was presented for the purpose of evaluating neurovascular treatments in the operating room. A series of studies were developed to assess the intravascular OCT system's ability to estimate flow. We demonstrated that the current intravascular system was suitable for DOCT imaging. Blood flow profiles were measured in flow phantoms and porcine models with confirmation of these results through CFD models. The development a novel DOCT method was then presented and evaluated through the same intravascular Doppler characterization experiments. This alternative method exhibited a lower Doppler noise floor compared to the conventional DOCT algorithm. Improved velocity sensitivity would allow for visualization of low velocity regions around treatment devices such as neurovascular stents. We demonstrated the application of DOCT imaging in stent apposition within the ICA. This imaging was performed before and after stent implantation, which showed dramatic change in the hemodynamics of the arterial vessel.

Intravascular ssDOCT imaging also demonstrates a potential clinical application in intracranial aneurysm treatment. Both the hemodynamics and morphological changes are believed to contribute to the rupture of an intracranial aneurysm. Furthermore, DOCT imaging during treatment of aneurysm could provide an insight into post procedural complications, as well as the flow conditions during vascular remodeling.

In summary, the following scientific contributions were made to attempt to provide an imaging method for physicians to evaluate neurovascular treatments.

- It can be observed in this thesis that significant efforts were focused on developing methods to accurately estimate blood velocity with an intravascular OCT catheter. Prior to this work, limited demonstration of DOCT imaging was reported. Custom acquisition hardware was constructed to collect the OCT interference signal from the detectors of the intravascular OCT system. Flow 
phantom studies were devised to characterize the velocity dynamic range as well as a blood-insaline contrast agent to resolve the moving scatterers within the blood vessel. Furthermore, we also observed that insufficient A-line sampling would result in the inability to resolve Doppler phase shifts. Initial in vivo experiments were then developed to demonstrate DOCT imaging within the carotid artery through conventional techniques. The results of this experiment was, to the best of our knowledge, the first demonstration of in vivo DOCT imaging with an intravascular rotary catheter OCT probe.

- The aforementioned conventional DOCT technique showed that intravascular DOCT imaging was feasible on a commercial OCT system. However, it was observed that Doppler phase noise would still exist within the DOCT images, resulting in phase estimate outliers from the expected result. Traditional averaging is commonly employed to resolve this noise; however it would lead to degradation of resolution. As a result, an alternative method was developed to improve phase estimates and sensitivity without significant degradation of velocity resolution. The approach presented in this thesis was a novel signal processing technique that split the interferogram of the detected OCT signal into multiple bands and was termed ssDOCT. Various parameters associated with ssDOCT were tested to provide optimal performance. Comparative analysis of ssDOCT and conventional DOCT imaging demonstrated increased suppression of the phase estimate outliers with the ssDOCT method.

- The first application of ssDOCT was utilized in carotid stenting. Three distinct flow patterns were visualized after stent placement. Low to stagnant low regions were observed in malapposition stent deployment or protruded stent struts at a bifurcation junction in the ICA. CFD simulation provided additional information of the local hemodynamics within the blood vessel. A number of studies have suggested that these regions have an increased risk in the formation of thrombosis, which in turn could lead to a stroke or other post-operative complications. Stent struts that are sparsely placed on the lumen wall had no significant effect on the main flow profile of the vessel. However, stent struts that were on the wall and densely clustered consisted of low flow velocities in between the struts, which in turn could promote thrombus formation. The clinical utility for identifying these low flow regions could provide potential risk of post-operative complications.

- The factors leading to the formation and rupture of an intracranial aneurysm is currently not well understood. It is believed that the these factors are due to the combination between the local interaction of blood with the arterial wall and the morphological compensatory mechanism to maintain the arterial wall's structural integrity. As a result, intravascular ssDOCT imaging has the potential to evaluate both the state of the local hemodynamics and microstructure of the arterial wall. This technique would further provide additional insight to surgeons during intraoperative treatment of intracranial aneurysms. We presented, to the best of our knowledge, the first evaluation of flow dynamics in intracranial aneurysms with DOCT imaging. Both ideal and patient specific flow models were imaged. CFD simulation were also employed for both scenarios. Similarities were observed in both the DOCT imaging and CFD models for ideal and patient 
specific flow models. Key flow events were observed through DOCT, which suggest the presence of recirculation zones or vortices. These events are believed to be cause of thrombus deposition and could lead to stroke.

- Another potential metric for evaluation of intracranial aneurysm rupture is the microstructural changes that are believed to occur due to vascular remodelling of the artery. Structural OCT imaging of 28 resected aneurysms from 21 patients were acquired. To our knowledge, this is the largest human ex vivo study to identify key morphological structures that are associated with intracranial aneurysm rupture. We demonstrated that structural OCT had the capability to identify key features such as the presence of cellular fibrotic tissue, paucicellular hyaline, and calcification. The formation of thrombosis was apparent in several of the specimen which may be a result of damaged endothelium from the blood flow. Further identification of these layers was demonstrated using OCT-OA. We had initially utilized this technique on medulloblastoma tissue. This is also believed to be the first evaluation of medulloblastoma with OCT imaging. The ability to differentiate the composition of the tissue could assist in the risk assessment of intracranial aneurysm rupture or potential post-operative complications.

\subsection{Future work}

During this $\mathrm{PhD}$, there was widespread development of DOCT platforms and structural OCT algorithms. The systems and methods that were presented in this thesis are the initial phase to translate intravascular DOCT imaging to the surgical operating room. However, several technological hurdles and scientific questions remain.

As previously mentioned in Chapter 3, the formation of thrombi along the stent struts relative to its placement is still unknown. In this work, we demonstrated the potential ability to evaluate the local hemodynamics of healthy blood vessels. Longitudinal analysis of stent placement and its risk of complication is necessary. Stent malapposition may lead to low velocity within the blood vessel and form regions of aggregated blood over a period of time, possibly leading to microemboli. This might have been the case for a 74-year-old male with atherosclerotic plaque in his carotid artery. It was decided that a 10 $\mathrm{mm}$ diameter stent would be deployed in the artery for treatment. However, during the deployment of the stent, it was unknown if the stent strut was correctly appositioned. Standard of care imaging could not provide the appropriate indication of stent malapposition. The postoperative CTA of the patient's carotid artery (Figure 6.1) suggested stent malapposition. Unfortunately, the patient lost vision in his left eye shortly after the operation, potentially due to a microemboli. This is one particular case where intravascular ssDOCT could have been used to assess the stent deployment. Currently, clinical trials are being developed to introduce intravascular ssDOCT imaging during angioplasty or stent interventions.

To understand the hemodynamic effect of clots in a stented artery, coagulated blood was injected into a stented carotid artery in a porcine model. The coagulated blood had attached to the stent struts and is believed to have artificially simulated large clot formation along the stented arterial vessel wall. The erratic flow generated from the clots near the lumen wall can result in turbulent flow within the 


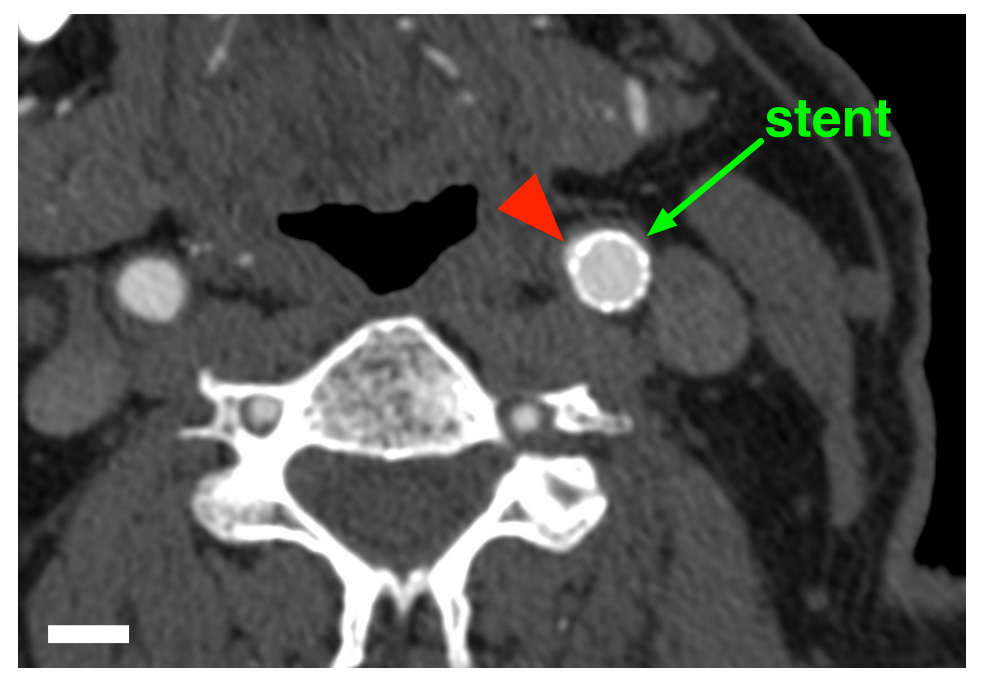

Figure 6.1: A computer tomography image of a 74-year-old male after stenting. It was observed that the stent was not completely deployed on the carotid artery wall (arrow). Scale bar: $1 \mathrm{~cm}$.

vessel. If enough stress is applied onto the wall due to the turbulent flow, leading to endothelium lesions. Previous studies have suggested that this could be a factor in restenosis [74]. This artificially simulated study does not account for the vascular compensatory mechanism, which would effect the flow patterns within the blood vessel.

a)

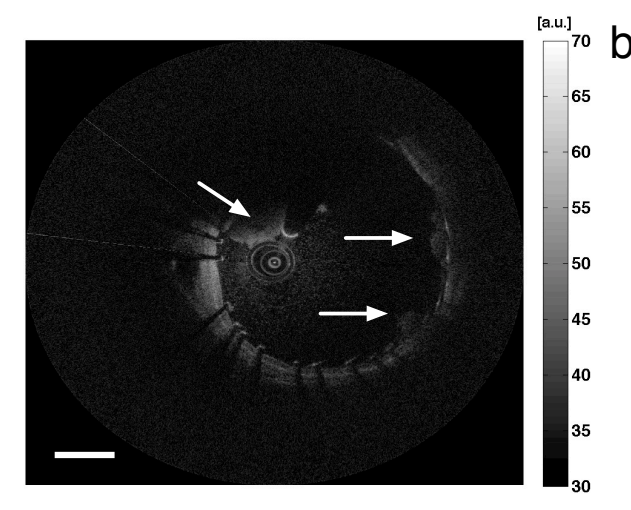

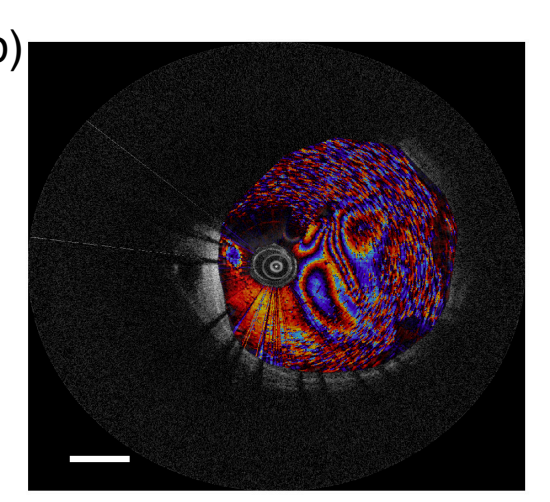

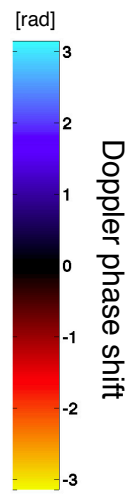

Figure 6.2: (a) Structural OCT image of a stented carotid artery. Coagulated blood was artificially injected into the vessel and attached to the stent struts (arrows). (b) The corresponding ssDOCT image. Scale bar: $1 \mathrm{~mm}$.

The positioning of the OCT catheter and its influence within the blood vessel has not been fully investigated. The results presented in this thesis suggested minimal influence on the hemodynamics during DOCT imaging. However, various positioning of the OCT catheter within a controlled environment would be need to be characterized prior to translation into the clinical setting. It should be noted that 
the most optimal position of the OCT catheter within the blood vessel may be difficult to achieve during intervention. As a result, taking the position of the catheter into account during velocity estimates would increase the accuracy.

In previous chapters, structural OCT and OCT-OA imaging of intracranial aneurysms was presented. These images were acquired using a bench-top system; however, catheter based OCT and OCT-OA imaging would provide a practical method to evaluate the aneurysm in vivo. Calibration of the system would also be necessary to eliminate variation in the optical parameters between each catheter. Reduction of the overall size of the OCT catheter would be required; however, tortuous vessels would be a challenge, as advancement of the distal tip of the OCT catheter may result in its damage. Furthermore, the commercially available OCT catheter was designed to image coronary arteries. As a result, an alternative design of the OCT catheter would be necessary to increase the working distance in order to visualize flow closer to the sac of the aneurysm or side branch. Furthermore, the size of the catheter would need to decrease without sacrificing the structural integrity of the OCT probe.

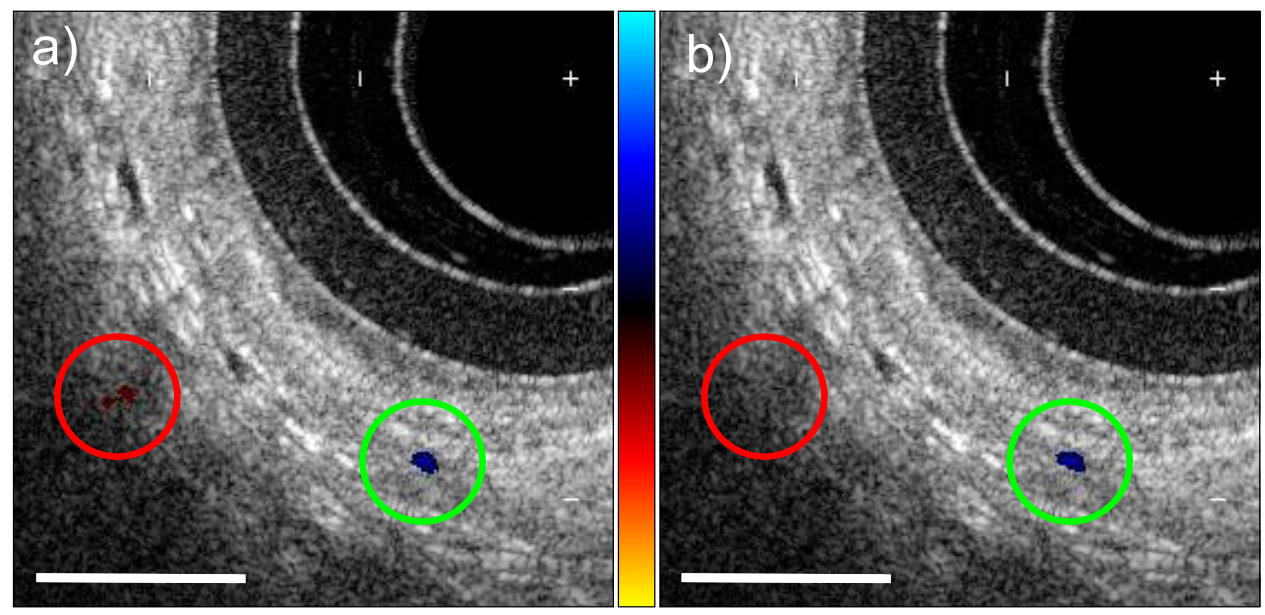

Figure 6.3: OCT imaging from an in-vivo human airway. (a) Structural OCT image with DOCT overlay $(\mathrm{B}=1, \mathrm{M}=8, \mathrm{~N}=32)$. (b) Split spectrum processing $(\mathrm{B}=4, \mathrm{M}=2, \mathrm{~N}=32, \mathrm{FBW}=60 \%)$ of the same imaging frame. Both images have identical Doppler display thresholds. The green circle indicates a true blood vessel confirmed by continuity between imaging frames. The red circle shows a Doppler artefact that was suppressed in the split spectrum processed image. The color-coded CDOCT scale bar spans +/-8.5 $\mathrm{mm} / \mathrm{s}$. Scale bar: $0.5 \mathrm{~mm}$ [4]. (Reprinted with permission of the Optical Society of America)

The application of ssDOCT could also be expanded to other vascular imaging fields. We had employed ssDOCT in a single case study in which the patient exhibited increase vascularture in the lung. In the imaged human airway, two low velocity blood vessels were detected using conventional OCT (Figure 6.3(a)). However, ssDOCT only resolved a single blood vessel. Confirmation of the existence of a single blood vessel was performed by observing the same feature in adjacent frames from a volumetric Doppler OCT image set. A longitudinal view of the blood vessel was also utilized to confirmed the presence of the single blood vessel (Figure 6.4). ssDOCT can be utilized to reduce false positive detection of small blood vessels. 


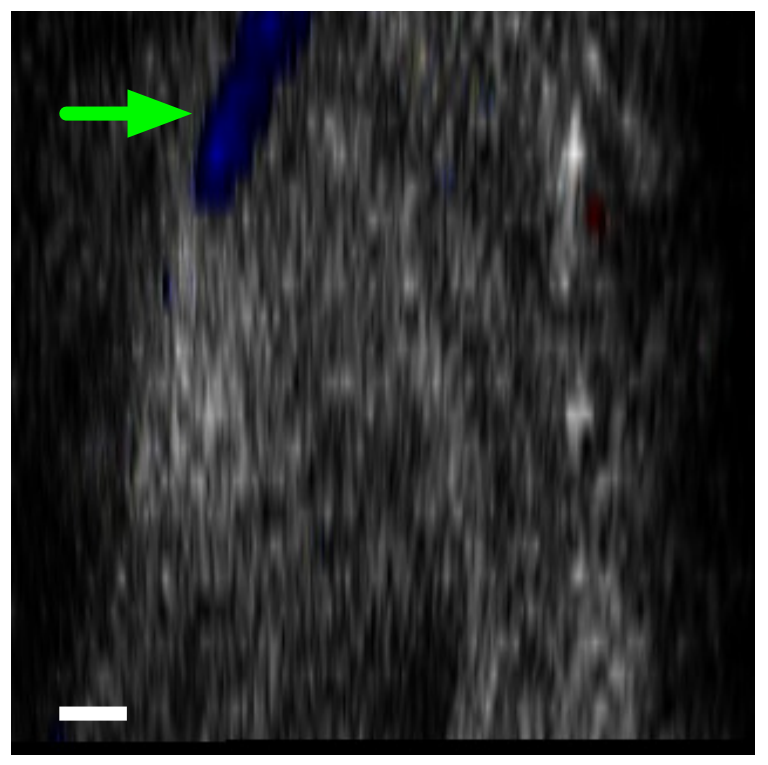

Figure 6.4: (a) A longitudinal view of in-vivo human airway. The Doppler image was overlaid onto the structural OCT image. A single blood vessel (arrow) could be clear resolved within the lung. Scale bar: $100 \mu \mathrm{m}$.

This research has generated DOCT advances, allowing for the potential for monitoring and quantification of stent apposition. It is envisioned that further progression of this research would lead to translation to clinical studies in the treatment of both atherosclerotic disease and intracranial aneurysm. Furthermore, the improvement of Doppler phase noise could lead to detection of highly vascular diseases such as cancer or HHT. 


\section{Appendix 1}

\section{Derivation of the Kasai velocity estimaton}

The received signal, $S(z)$, can be presented in complex form

$$
S(z)=x(z)+j y(z)
$$

where $x(z)$ is the in-phase or real component and $y(z)$ is the quadrature or imaginary component.

Let the power spectrum of $S(z)$ be $P(w)$, where the mean angular frequency of $P(w)$ is expressed as [39]

$$
\bar{w}=\frac{\int_{-\infty}^{\infty} w P(w) d w}{\int_{-\infty}^{\infty} P(w) d w},
$$

and the mean velocity is

$$
\bar{v}=\frac{\bar{w}}{w} \frac{c}{2 \cos (\theta)} .
$$

The relationship between the autocorrelation \& power spectrum. This is know as the WienerKhinchine theorem

$$
R(\tau)=\int_{-\infty}^{\infty} P(w) \exp (j w \tau) d w .
$$

Taking the derivative of $R_{g g}(\tau)$ gives

$$
\dot{R}(\tau)=\frac{d R_{g g}(\tau)}{d \tau}=\int_{-\infty}^{\infty} j w P(w) \exp (j w \tau) d w .
$$


Substituting Eq.1.5 into Eq.1.2

$$
\bar{w}=\frac{\int_{-\infty}^{\infty} w P(w) d w}{\int_{-\infty}^{\infty} P(w) d w}=\frac{1}{j} \frac{\dot{R}(0)}{R(0)} .
$$

As a result, it can be seen that the mean angular frequency is related to the autocorrelation at $\tau=0$

We can also represent the received signal as [62]

$$
\begin{gathered}
S(z)=a \cdot g(z) \exp \left(-j\left(2 \pi \frac{2 v_{z}}{c} f_{o} z\right)\right), \\
\phi_{v}=2 \pi \frac{2 v_{z}}{c} f_{o},
\end{gathered}
$$

where $f_{o}$ is the centre frequency, $g(z)$ is the envelop of the pulse, and $a$ is the amplitude.

The autocorrelation can be represented as

$$
\begin{gathered}
R(\tau)=\int_{-\infty}^{\infty} a \cdot g^{*}(z) \exp \left(j \phi_{v} z\right) a \cdot g(z-\tau) d z \\
=a^{2} \int_{-\infty}^{\infty} g(z) g(z-\tau) \exp \left(-j \phi_{v} \tau\right) d z \\
=a^{2} R_{g g}(\tau) \exp \left(-j \phi_{v} \tau\right)
\end{gathered}
$$

Taking the derivative of $R(\tau)$

$$
\dot{R}(\tau)=\frac{d R(\tau)}{d \tau}=a^{2}\left(\dot{R_{g g}}(\tau)-j \phi_{v} R_{g g}(\tau)\right) \exp \left(-j \phi_{v} \tau\right) .
$$

Expanding around $\tau=0$

$$
\left.\frac{d R(\tau)}{d \tau}\right|_{\tau=0}=a^{2}\left(\dot{R_{g g}}(0)-j \phi_{v} R_{g g}(0)\right)
$$

From Eq.1.5, $\dot{R_{g g}}(0)=0$. Therefore,

$$
\begin{gathered}
\dot{R}(0)=-a^{2} \cdot j \phi_{v} R_{g g}(0), \\
R(0)=a^{2} R_{g g}(0) .
\end{gathered}
$$


In this form, $\bar{w}$ is given as

$$
\bar{w}=\frac{1}{j} \frac{\dot{R}(0)}{R(0)}=\frac{1}{j} \cdot \frac{-a^{2} \cdot j \phi_{v} R_{g g}(0)}{a^{2} R_{g g}(0)}=-\phi_{v} .
$$

In practice, discrete autocorrelation is utilized and is given as [62]

$$
\hat{R(m)}=\lim _{x \rightarrow \infty} \frac{1}{2 N+1} \sum_{i=-N}^{N} r^{*}(i) r(i+m) .
$$

Let the received discrete signal be

$$
S(i)=x(i)+j y(i)
$$

and

$$
\begin{gathered}
r^{*}(i) r(i+m)=(x(i)-j y(i))(x(i+m)+j y(i+m)), \\
=x(i) \cdot x(i+m)+y(i) \cdot y(i+m)+j(y(i+m) \cdot x(i)-x(i+m) \cdot y(i)) .
\end{gathered}
$$

Generally a limited amount of data is present. As a result, using $N_{c}$ lines the estimate (Equation 1.17) becomes,

$$
\begin{gathered}
R \hat{(1)}=\frac{1}{N_{c}-1} \sum_{i=0}^{N_{c}-2} r^{*}(i) r(i+1), \\
R \hat{(1)}=\frac{1}{N_{c}-1} \sum_{i=0}^{N_{c}-2} x(i) \cdot x(i+1)+y(i) \cdot y(i+1)+j(y(i+1) \cdot x(i)-x(i+1) \cdot y(i)) .
\end{gathered}
$$

In ultrasound the lines estimates are acquired at $T_{p r f}$ which results in the following:

$$
R\left(T_{p r f}\right)=\frac{1}{N_{c}-1} \sum_{i=0}^{N_{c}-2} x(i) \cdot x(i+1)+y(i) \cdot y(i+1)+j(y(i+1) \cdot x(i)-x(i+1) \cdot y(i))
$$

Therefore taking the arctan to find $\phi_{v}$,

$$
\begin{gathered}
-\phi_{v}=\arctan \left\{\frac{\Im\left\{R\left(T_{p r f}\right)\right\}}{\Re\left\{R\left(T_{p r f}\right)\right\}}\right\}, \\
\phi_{v}=\arctan \left\{\frac{\frac{1}{N_{c}-1} \sum_{i=0}^{N_{c}-2} x(i+1) \cdot y(i)-y(i+1) \cdot x(i)}{\frac{1}{N_{c}-1} \sum_{i=0}^{N_{c}-2} x(i) \cdot x(i+1)+y(i) \cdot y(i+1)}\right\} .
\end{gathered}
$$




\section{Appendix 2}

\section{Derivation of the velocity variance}

From the previous Appendix,

$$
R(\tau)=\int_{-\infty}^{\infty} P(w) \exp (j w \tau) d w
$$

and

$$
\bar{w}=\frac{\int_{-\infty}^{\infty} w P(w) d w}{\int_{-\infty}^{\infty} P(w) d w}=\frac{1}{j} \frac{\dot{R}(0)}{R(0)} .
$$

The variance is define as $[39,62]$

$$
\begin{aligned}
\sigma^{2} & =\frac{\int_{-\infty}^{\infty}(w-\bar{w})^{2} P(w) d w}{\int_{-\infty}^{\infty} w P(w) d w} \\
= & \frac{\int_{-\infty}^{\infty} w^{2} P(w) d w}{\int_{-\infty}^{\infty} P(w) d w}-\bar{w}^{2}
\end{aligned}
$$

Taking the first and second derivative of Eq.2.1 gives,

$$
\dot{R}(\tau)=\int_{-\infty}^{\infty} j w P(w) \exp (j w \tau) d w
$$


and

$$
\ddot{R}(\tau)=\int_{-\infty}^{\infty}-w^{2} P(w) d w .
$$

Then

$$
\sigma^{2}=\left(\frac{\dot{R}(0)}{R(0)}\right)^{2}-\frac{\ddot{R}(0)}{R(0)} .
$$

The autocorrelation with respect to the envelop pulse is

$$
R(\tau)=a^{2} R_{g g}(\tau) \exp \left(-j \phi_{v} \tau\right) .
$$

The first and second derivative is given by

$$
\begin{gathered}
\dot{R}(\tau)=a^{2}\left(\dot{R_{g g}}(\tau)-j \phi_{v} R_{g g}(\tau)\right) \exp \left(-j \phi_{v} \tau\right), \\
\ddot{R}(\tau)=a^{2}\left(\left[\ddot{R_{g g}}(\tau)-j \phi_{v} \dot{R_{g g}}(\tau)\right] \exp \left(-j \phi_{v} \tau\right)+j \phi_{v}\left[\dot{R_{g g}}(\tau)-j \phi_{v} R_{g g}(\tau)\right] \exp \left(-j \phi_{v} \tau\right)\right) .
\end{gathered}
$$

Evaluating the first and second derivative at $\tau=0$ gives,

$$
\dot{R}(0)=a^{2}\left(\dot{R_{g g}}(0)-j \phi_{v} R_{g g}(0)\right)
$$

and

$$
\ddot{R}(0)=a^{2}\left(\ddot{R_{g g}}(0)-2 j \phi_{v} \dot{R_{g g}}(0)-\phi_{v}^{2} R_{g g}(0)\right) .
$$

Substituting Eq.2.11 and Eq.2.12 into Eq.2.7 gives,

$$
\sigma^{2}=-\phi_{v}^{2}-\frac{a^{2} \ddot{R_{g g}}(0)-a^{2} \phi_{v} R_{g g}(0)}{a^{2} R_{g g}(0)} .
$$

Since $\dot{R_{g g}}(0)=0$ then the equation becomes,

$$
\sigma^{2}=-\frac{\ddot{R_{g g}}(0)}{R_{g g}(0)} .
$$

Making a Taylor expansion approximation of $R_{g g}(\tau)$ around $\tau=0$ gives,

$$
R_{g g}(\tau) \approx R_{g g}(0)+\frac{\tau^{2}}{2} \ddot{R_{g g}}(0)+\ldots .
$$

If we neglect the higher orders then,

$$
R_{g g}(\tau)=R_{g g}(0)+\operatorname{frac}^{2} 2 \ddot{R_{g g}}(0),
$$


and

$$
\ddot{R_{g g}}(0)=\left(R_{g g}(\tau)-R_{g g}(0)\right) \frac{2}{\tau^{2}} .
$$

Substituting Eq.2.16 and Eq.2.17 into Eq.2.14 gives,

$$
\sigma^{2} \approx \frac{R_{g g}(0)-R_{g g}(\tau)}{R_{g g}(0)} \cdot \frac{2}{\tau^{2}}
$$

In ultrasound, the time between two pulse to measure a moving scatter is denoted at $T_{p r f}$. Therefore, we let $\tau=T_{p r f}$ leading to the following,

$$
\sigma^{2} \approx \frac{2}{T_{p r f}^{2}}\left(1-\frac{\left|R_{g g}\left(T_{p r f}\right)\right|}{R_{g g}(0)}\right)
$$




\section{Appendix 3}

\section{Tissue handling protocol}

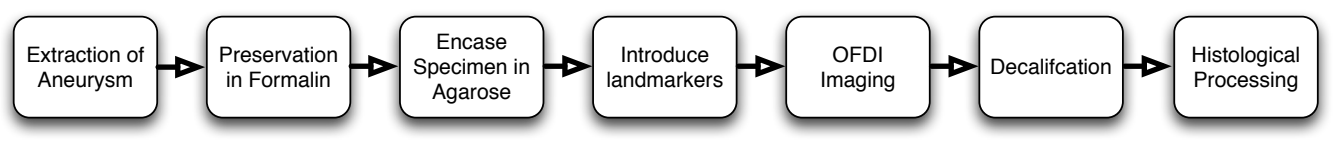

Figure 3.1: Work flow chart of the tissue preparation and imaging used in this study. [6]

\section{a)}

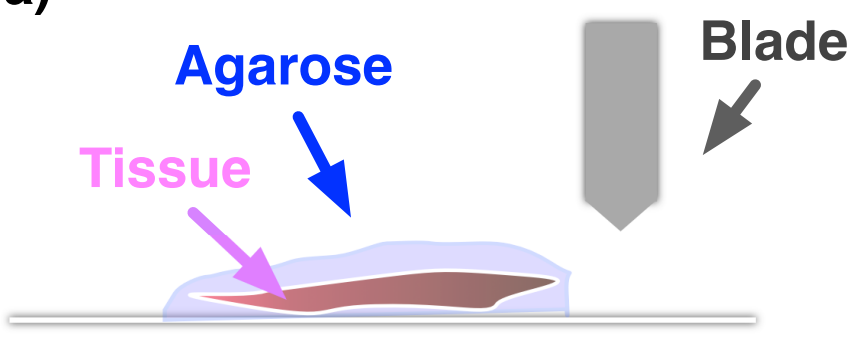

b)

\section{Scanning Direction}

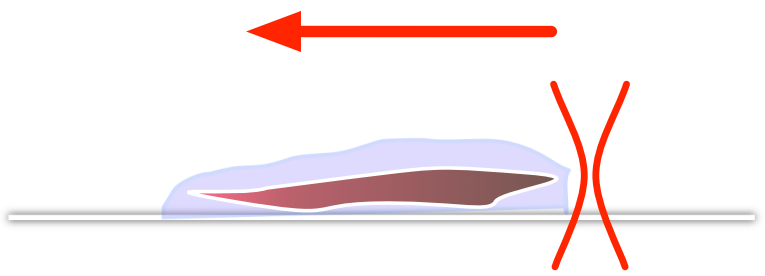

Figure 3.2: (a) Fiducial markers were cut into the agarose. (b) This represented starting position of the OCT scan. 


\section{Appendix 4}

\section{Optical coherence tomography - optical attenuation flow diagram}

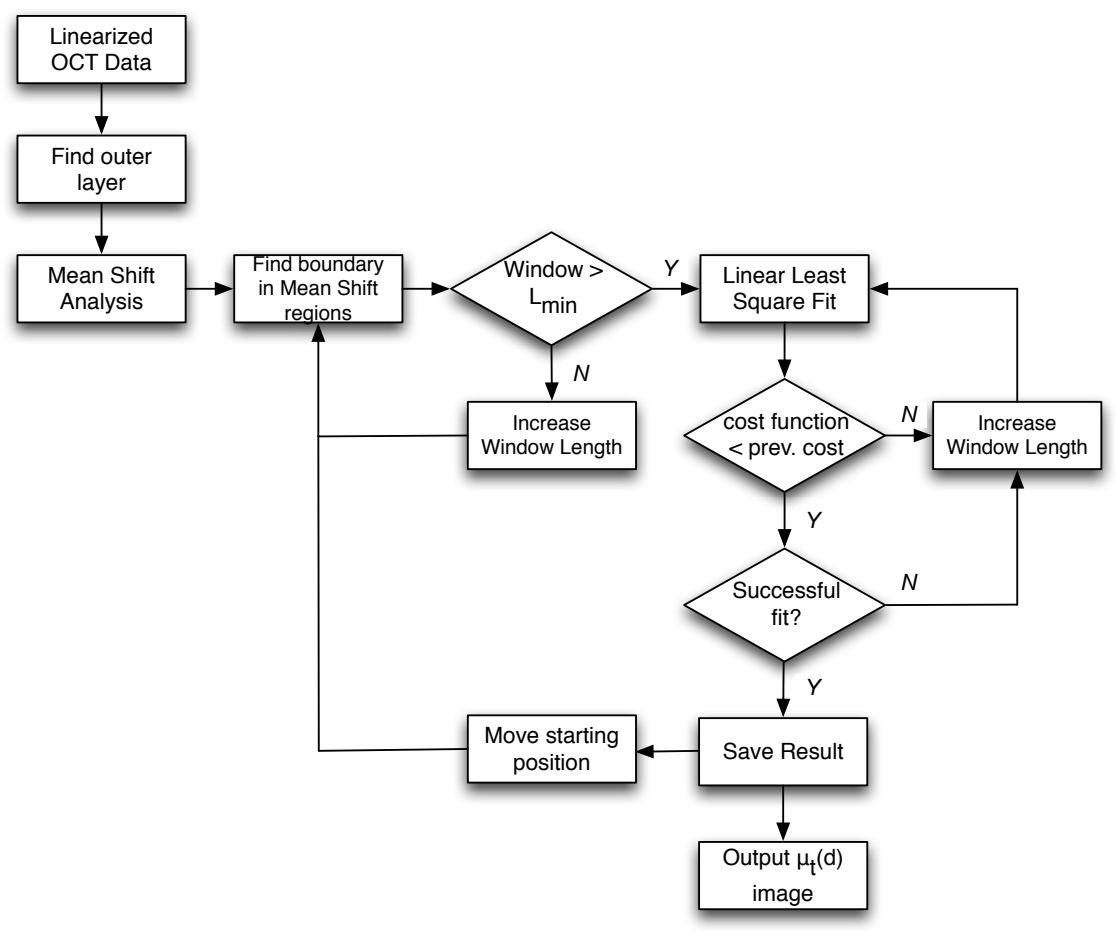

Figure 4.1: Flow diagram of a mean shift image segmentation based optical coherence tomography optical attenuation imaging 


\section{Appendix 5}

\section{Permission}

\subsection{The Optical Society of America (Optics Express and Biomed- ical Optics Express)}

On Mon, Mar 23, 2015 at 4:50 PM, pubscopyright <copyright@osa.org> wrote:

Dear Mr. Vuong,

Thank you for contacting The Optical Society.

Because you are the author of the source paper from which you wish to reproduce material, OSA considers your requested use of its copyrighted materials to be permissible within the author rights granted in the Copyright Transfer Agreement submitted by the requester on acceptance for publication of his/her manuscript. It is requested that a complete citation of the original material be included in any publication. This permission assumes that the material was not reproduced from another source when published in the original publication.

Please let me know if you have any questions.

Kind Regards,

Susannah Lehman

March 23, 2015

Authorized Agent, The Optical Society 


\section{Appendix 6}

\section{List of Contributions}

\section{Journal Publications Primary to PhD Work}

1. Vuong, B., Ramjist, J., Genis, H., Jivraj, J., Yang, Wong, R., V.X.D. (2015) Evaluation of hemodynamics in intracranial aneurysms through split spectrum Doppler optical coherence tomography and computational modeling techniques. American Journal of Neuroradiology, Submission: 1500417 (Submitted).

2. Vuong, B., Gardecki, J.A., Kiehl, T., Standish, B.A., Sun, C., da Costa, L., de Morais, J.V., Tearney, G.K., Yang, V.X.D. (2014) Feasibility of the assessment of human intracranial aneurysm with Optical Frequency Domain Imaging. Journal of Neurosurgery. Submission: JNS14-2840 (Submitted).

3. Vuong, B., Genis, H., Wong, R., Ramjist, J., Jivraj, J., Farooq, H., Sun, C., Yang, V.X.D. (2014) Evaluation of flow velocities after carotid artery stenting through split spectrum Doppler optical coherence tomography and computational fluid dynamics modeling. Biomedical Optics Express. 6:1487-1501.

4. Vuong, B., Skowron, P., Kiehl, TR., Kyan, M., Garzia, L., Sun, C., Talyor, M.D., Yang, Y.X.D. (2014) Measuring the optical characteristics of medulloblastoma using optical coherence tomography. Biomedical Optics Express. 5:4405-4416.

5. Vuong, B., Lee, A.M.D., Luk, T.W.H., Sun, C., Lam, S., Lane, P., Yang, V.X.D. (2014) High speed, wide velocity dynamic range Doppler optical coherence tomography (Part IV): split spectrum processing in rotary catheter probes. Optics Express. 22:7399-7415.

\section{Collaborative Journal Publications Related to PhD Work}

1. Huang, Y., Jivraj, J., Ramjist, J., Wong, R., Lu, Y., Vuong, B., Sun, C., Gu, X., Yang, V.X.D. (2014) Compact coaxial cascaded optical coherence tomography and fiber laser ablation system. 
Optics Express. Submission: 224442 (Submitted).

2. Wong, R., Jivraj, J., Vuong, B., Ramjist, J., Dinn, N.A., Sun, C., Huang, Y., Smith, J.A., Yang, V.X.D. (2014) Development of an integrated optical coherence tomography-gas nozzle system for surgical laser ablation applications: preliminary findings of in-situ spinal cord deformation due to gas flow effects. Biomedical Optics Express. 6:43-53.

3. Cheng, K. H., Mariampillai, A., Lee, K. K., Vuong, B., Luk, T. W., Ramjist, J., Curtis, A., Jakubovic, H., Kertes, P., Letarte, M., Faughnan M.E., Brain Vascular Malformation Group Consortium HHT Investigator Group, Yang, V. X.D. (2014) Histogram flow mapping with optical coherence tomography for in vivo skin angiography of hereditary hemorrhagic telangiectasia. Journal of Biomedical Optics.19:086015-086015.

4. Sun, C., Standish, B., Vuong,B., Wen, X.Y., Yang,V.X.D. (2013) Digital image correlation -based optical coherence elastography. Journal of Biomedical Optics. 18:121515-121515.

5. Wawrzyn, K. Demidov, V., Vuong, B., Harduar, M.K., Yang, V.X.D., Doganay O., Toronov, V. Xu,Y., (2013) Imaging the electro-kinetic response of biological tissues with optical coherence tomography. Optics Letters. 38:2572-25774.

6. Mahumd, M.S., Codotte, D.W., Vuong, B., Sun, C., Luk, T.W.H., A., Mariampillai, A., Yang, V.X.D. (2013) A Review of Speckle and Phase Variance Optical Coherence Tomography to Visualize Microvascular Networks. Journal of Biomedical Optics. 18:050901 .

7. Sun, C., Nolte, F., Vuong, B., Lee, K.K.C, Standish, B.A., Courtney, B., Standish, B.A., Marotta, T.R., A., Mariampillai, A., Yang, V.X.D. (2012) In vivo feasibility of endovascular Doppler optical coherence tomography. Biomedical Optics Express 3:2600-2610.

8. Sun, C., Lee, K.K.C. Vuong, B., Cusimano, M., Brukson, A., Mariampillai, A., Standish, B.A., Yang, V.X.D. (2012) Neurosurgical hand-held optical coherence tomography forward view probe. Biomedical Optics Express. 3:1404-1412.

9. Cheng, K.H.Y., Sun, C., Vuong, B., Lee, K.K.C. and Mariampillai, A., Marotta, T.R., Spears, J.,Montanera, W.J., Herman, P.R., Kiehl, T.R., Yang, V.X.D. (2012) Endovascular optical coherence tomography intensity kurtosis: visualization of vasa vasorum in porcine carotid artery, Biomedical Optics Express. 3:388-399.

10. Harduar, M.K., Mariampillai, A., Vuong, B., Gu, X., Standish, B.A., and Yang, V.X.D. (2011) Dual-core ytterbium doped fiber amplifier for high-power $1060 \mathrm{~nm}$ swept source multichannel optical coherence tomography. Optics Letter 36:2976-2978. 


\section{Refereed Conference Proceedings Primary to PhD Work}

1. Vuong, B., Genis, H., Wong, R., Ramjist, J., Jivraj, J., Sun, C., Yang, V.X.D. (2015)Visualization of blood flow in carotid artery stenting with endovascular Doppler optical coherence tomography imaging and computational fluid dynamic modeling. Proceedings of the PSFVIP-10 (Accepted).

2. Vuong, B., Genis, H., Wong, R., Ramjist, J., Jivraj, J., Sun, C., Yang, V.X.D. (2015) Evaluation of hemodynamics changes during interventional stent placement using Doppler optical coherence tomography. Proceedings of the SPIE Photonics West. 93050Q-93050Q.

3. Vuong, B., Skowron, P., Kiehl, T.R., Kyan, M., Garzia, L., Genis,H., Sun, C., Taylor, M.D., and Yang. V.X.D. (2015) Application of optical coherence tomography attenuation imaging for quantification of optical properties in medulloblastoma. Proceedings of the SPIE Photonics West, 93122Z-93122Z.

4. Vuong, B., Genis, H., Ramjist, J., Wong, R., Jivraj, J., Yang, V.X.D. (2015) Patient specific intracranial aneurysm flow phantom imaging with optical coherence tomography Proceedings of the SPIE Photonics West. Submission: 9305-126 (Accepted).

5. Vuong, B., Sun, C., Kiehl, T., Gardecki, J.A., Standish, B.A., da Costa, L., de Morais, J.V., Tearney, G.K., Yang, V.X.D. (2012) Feasibility and methodology of optical coherence tomography imaging of human intracranial aneurysm: ex vivo pilot study. Proceedings of the SPIE Photonics West. 82074M-82074M-8.

6. Vuong, B., Standish, B., Sun, C., Tearney, G., Kiehl, R., van der Kwast, T., Koo, J., Wilson, B.C., de Morais, J., da Costa, L., and Yang V.X.D. (2011) 3D Optical Coherence Tomography and Digital Pathology. Conference on Lasers and Electro-Optics/Pacific Rim. C353.

7. Vuong, B., Harduar, M.K., Mariampillai, A., Isamoto, K., Chong, C., Standish, B.A., and Yang, V.X.D. (2011) $23 \mathrm{kHz}$ MEMS based swept source for optical coherence tomography imaging. Proceedings of the 33rd IEEE Engineering in Medicine and Biology Conference. 6134-6137.

8. Vuong, B., Harduar, M.K., Cheng, K.H.Y., Gu, X., Chen, L.R., Standish, B.A., and Yang, V.X.D. (2010) Cascaded Raman Fiber Laser in Fourier Domain Mode Lock Operation. Proceedings of the SPIE Photonics West. 7580-101.

\section{Refereed Conference Proceedings Related to PhD Work}

1. Jivraj, J., Huang, Y., Wong, R., Lu,Y., Vuong, B., Ramjist, J., Gu, X., and Yang. V.X.D., (2015) Development of a low cost, 3-DOF desktop laser cutter using 3D printer hardware. Proceedings of the SPIE Photonics West. 93560N-93560N .

2. Jivraj, J., Huang, Y., Wong, R., Lu,Y., Vuong, B., Ramjist, J., Gu, X., and Yang. V.X.D., (2015) Coaxial cavity injected OCT and fiber laser ablation system for real-time monitoring of ablative processes. Proceedings of the SPIE Photonics West. 930505-930505. 
3. Wong, R., Jivraj, J., Vuong, B., Ramjist, J., Sun, C., Huang, Y., and Yang. V.X.D., (2015) Spinal cord deformation due to nozzle gas flow effects using optical coherence tomography. Proceedings of the SPIE Photonics West. 93050A-93050A.

4. Toronov,V.,Demidov, V., Xu.,Y., Vuong, B., Sun, C., Yang, V.X.D. (2014) Vitkin, A. (2014) Imaging of electro-kinetic properties of tissue using the amplitude and the phase of optical coherence tomography. Proceedings of the SPIE Photonics West. 89420X-89420X-14.

5. Sun, C., Vuong, B., Wen, X., Yang., V.X.D. (2013) Preliminary study of digital image correlation based optical coherence elastography Proceedings of European Conferences on Biomedical Optics. 8802:880207-5 .

6. Demidov, V., Toronov, V. Xu.,Y., Vuong, B., Sun, C., Yang, V.X.D. (2013) Vitkin, A., Imaging the electro-kinetic response of biological tissues with optical coherence tomography Proceedings of the SPIE Photonics West. 85712Q-85712Q-10.

7. Sun, C., Nolte, F., Vuong, B., Lee, K.K.C, Standish, B.A., Courtney, B., Standish, B.A., Marotta, T.R., A., Mariampillai, A., Yang, V.X.D. (2013) Blood flow velocity measurement by endavascular Doppler optical coherence tomography. Proceedings of the SPIE Photonics Wes. 85654S-85654S-6.

8. Wawrzyn, K. Vuong, B., Harduar, M.K., Yang, V.X.D., Toronov, V. Xu.,Y. (2012) Monitoring Electric Current in Biological Tissues by Optical Coherence Tomography, Proceedings of the Biomedical Optics. BW2A. 4.

9. Sun, C., Lee, K.K.C. Vuong, B., Cusimano, M., Brukson, A., Mariampillai, A., Standish, B.A., Yang, V.X.D. (2012) Neurosurgical hand-held optical coherence tomography forward view probe. Proceedings of the SPIE Photonics West. 82074V-82074V-8.

10. Harduar, M.K., Mariampillai, A., Vuong, B., Cheng, K.H.Y.,Chen, L.R., Gu, X., Standish, B.A., and Yang, V.X.D. (2010) Developing dual core ytterbium doped fiber ring laser in Fourier Domain Mode Lock Operation. Proceedings of the SPIE Photonics West 7580-102.

\section{Book Chapter Related to PhD Work}

11. Farooq, H., Genis, H., Alarcon, J., Vuong, B. Jivraj, J., Yang, V.X.D., Cohen-Adad, J., Fehlings, M.G., Cadotte, D.W., (2015) High-resolution imaging of the central nervous system: how novel imaging methods combined with navigation strategies will advance patient care. In Numa Dancause, Serge Rossignol, Sylvie Nadeau, editors: Sensorimotor Rehabilitation: At the Crossroads of Basic and Clinical Sciences 1st edition, Elsevier, 55-78. 


\section{References}

[1] B. Vuong, P. Skowron, T.-R. Kiehl, M. Kyan, L. Garzia, C. Sun, M. D. Taylor, and V. X. D. Yang, "Measuring the optical characteristics of medulloblastoma with optical coherence tomography," Biomedical Optics Express, vol. 6, no. 4, pp. 1487-1501, 2015.

[2] V. X. D. Yang, M. Gordon, B. Qi, J. Pekar, S. Lo, E. Seng-Yue, A. Mok, B. Wilson, and I. Vitkin, "High speed, wide velocity dynamic range doppler optical coherence tomography (part i): System design, signal processing, and performance," Optics Express, vol. 11, no. 7, pp. 794-809, 2003.

[3] C. Sun, F. Nolte, K. H. Cheng, B. Vuong, K. K. Lee, B. A. Standish, B. Courtney, T. R. Marotta, A. Mariampillai, and V. X. D. Yang, "In vivo feasibility of endovascular doppler optical coherence tomography," Biomedical Optics Express, vol. 3, no. 10, pp. 2600-2610, 2012.

[4] B. Vuong, A. Lee, T. W. H. Luk, C. Sun, S. Lam, P. Lane, and V. X. D. Yang, "High speed, wide velocity dynamic range doppler optical coherence tomography (part iv): split spectrum processing in rotary catheter probes," Optics Express, vol. 22, no. 7, pp. 7399-7415, 2014.

[5] B. Vuong, H. Genis, R. Wong, J. Ramjist, J. Jivraj, H. Farooq, C. Sun, and V. X. D. Yang, "Evaluation of flow velocities after carotid artery stenting through split spectrum doppler optical coherence tomography and computational fluid dynamics modeling," Biomedical Optics Express, vol. 5, no. 12, pp. 4405-4416, 2014.

[6] B. Vuong, C. Sun, T.-R. Kiehl, J. A. Gardecki, B. A. Standish, L. da Costa, J. de Morais, G. J. Tearney, and V. X. D. Yang, "Feasibility and methodology of optical coherence tomography imaging of human intracranial aneurysms: ex vivo pilot study," in SPIE BiOS. International Society for Optics and Photonics, 2012, pp. 82 074M-82 074M.

[7] D. Krex, H. Schackert, and G. Schackert, "Genesis of cerebral aneurysms-an update," Acta Neurochirurgica, vol. 143, no. 5, pp. 429-449, 2001.

[8] S. Natarajan, L. Sekhar, B. Ghodke, G. Britz, D. Bhagawati, and N. Temkin, "Outcomes of ruptured intracranial aneurysms treated by microsurgical clipping and endovascular coiling in a high-volume center," American Journal of Neuroradiology, vol. 29, no. 4, pp. 753-759, 2008. 
[9] J. Humphrey and P. Canham, "Structure, mechanical properties, and mechanics of intracranial saccular aneurysms," Journal of Elasticity and the Physical Science of Solids, vol. 61, no. 1-3, pp. 49-81, 2000.

[10] B. Weir, "Unruptured intracranial aneurysms: a review," Journal of Neurosurgery, vol. 96, no. 1, pp. 3-42, 2002.

[11] D. O. Wiebers, I. S. of Unruptured Intracranial Aneurysms Investigators et al., "Unruptured intracranial aneurysms: natural history, clinical outcome, and risks of surgical and endovascular treatment," The Lancet, vol. 362, no. 9378, pp. 103-110, 2003.

[12] S. L. Giannotta and N. S. Litofsky, "Reoperative management of intracranial aneurysms," Journal of Neurosurgery, vol. 83, no. 3, pp. 387-393, 1995.

[13] T. Lin, A. J. Fox, and C. G. Drake, "Regrowth of aneurysm sacs from residual neck following aneurysm clipping," Journal of Neurosurgery, vol. 70, no. 4, pp. 556-560, 1989.

[14] T. Sakaki, T. Takeshima, M. Tominaga, H. Hashimoto, and S. Kawaguchi, "Recurrence of ica-pcoa aneurysms after neck clipping," Journal of Neurosurgery, vol. 80, no. 1, pp. 58-63, 1994.

[15] K. Tsutsumi, K. Ueki, A. Morita, M. Usui, and T. Kirino, "Risk of aneurysm recurrence in patients with clipped cerebral aneurysms results of long-term follow-up angiography," Stroke, vol. 32, no. 5, pp. 1191-1194, 2001.

[16] A. J. Molyneux, R. S. Kerr, L.-M. Yu, M. Clarke, M. Sneade, J. A. Yarnold, and P. Sandercock, "International subarachnoid aneurysm trial (isat) of neurosurgical clipping versus endovascular coiling in 2143 patients with ruptured intracranial aneurysms: a randomised comparison of effects on survival, dependency, seizures, rebleeding, subgroups, and aneurysm occlusion," The Lancet, vol. 366, no. 9488, pp. 809-817, 2005.

[17] C. O'Kelly, J. Spears, M. Chow, J. Wong, M. Boulton, A. Weill, R. Willinsky, M. Kelly, and T. Marotta, "Canadian experience with the pipeline embolization device for repair of unruptured intracranial aneurysms," American Journal of Neuroradiology, vol. 34, no. 2, pp. 381-387, 2013.

[18] J. Y. Streifler, M. Eliasziw, A. J. Fox, O. R. Benavente, V. C. Hachinski, G. G. Ferguson, and H. Barnett, "Angiographic detection of carotid plaque ulceration. comparison with surgical observations in a multicenter study. north american symptomatic carotid endarterectomy trial." Stroke, vol. 25, no. 6, pp. 1130-1132, 1994.

[19] P. A. McCullough, A. Adam, C. R. Becker, C. Davidson, N. Lameire, F. Stacul, and J. Tumlin, "Epidemiology and prognostic implications of contrast-induced nephropathy," The American Journal of Cardiology, vol. 98, no. 6, pp. 5-13, 2006.

[20] R. A. Willinsky, S. M. Taylor, K. TerBrugge, R. I. Farb, G. Tomlinson, and W. Montanera, "Neurologic complications of cerebral angiography: Prospective analysis of 2,899 procedures and review of the literature 1," Radiology, vol. 227, no. 2, pp. 522-528, 2003. 
[21] T. J. Kaufmann, J. Huston III, J. N. Mandrekar, C. D. Schleck, K. R. Thielen, and D. F. Kallmes, "Complications of diagnostic cerebral angiography: Evaluation of 19826 consecutive patients 1," Radiology, vol. 243, no. 3, pp. 812-819, 2007.

[22] T. G. Brott, R. W. Hobson, G. Howard, G. S. Roubin, W. M. Clark, W. Brooks, A. Mackey, M. D. Hill, P. P. Leimgruber, A. J. Sheffet et al., "Stenting versus endarterectomy for treatment of carotid-artery stenosis," New England Journal of Medicine, vol. 363, no. 1, pp. 11-23, 2010.

[23] D. G. Grosset, D. Georgiadis, A. W. Kelman, P. Cowburn, S. Stirling, K. R. Lees, A. Faichney, A. Mallinson, R. Quin, I. Bone et al., "Detection of microemboli by transcranial doppler ultrasound." Texas Heart Institute Journal, vol. 23, no. 4, p. 289, 1996.

[24] D. W. Rowed, D. A. Houlden, L. M. Burkholder, and A. B. Taylor, "Comparison of monitoring techniques for intraoperative cerebral ischemia," The Canadian Journal of Neurological Sciences, vol. 31, no. 3, pp. 347-356, 2004.

[25] J. D. Kirsch, M. Mathur, M. H. Johnson, G. Gowthaman, and L. M. Scoutt, "Advances in transcranial doppler us: Imaging ahead," Radiographics, vol. 33, no. 1, pp. E1-E14, 2013.

[26] M. Gorman, "Transcranial doppler assessment of cerebral embolic disorders," Progress in cardiovascular diseases, vol. 43, no. 2, pp. 135-150, 2000.

[27] C. Jansen, E. Vriens, B. Eikelboom, F. Vermeulen, J. Van Gijn, and R. Ackerstaff, "Carotid endarterectomy with transcranial doppler and electroencephalographic monitoring. a prospective study in 130 operations." Stroke, vol. 24, no. 5, pp. 665-669, 1993.

[28] V. X. D. Yang and I. A. Vitkin, "Principles of doppler oct," Chapter, vol. 32, pp. 305-318, 2007.

[29] R. Stendel, T. Pietilä, A. A. Al Hassan, A. Schilling, and M. Brock, "Intraoperative microvascular doppler ultrasonography in cerebral aneurysm surgery," Journal of Neurology, Neurosurgery $\mathbb{E}$ Psychiatry, vol. 68, no. 1, pp. 29-35, 2000.

[30] C. Scholda, M. Wirtitsch, B. Hermann, A. Unterhuber, E. Ergun, H. Sattmann, T. H. Ko, J. G. Fujimoto, A. F. Fercher, M. Stur et al., "Ultrahigh resolution optical coherence tomography of macular holes," Retina, vol. 26, no. 9, pp. 1034-1041, 2006.

[31] G. J. Tearney, H. Yabushita, S. L. Houser, H. T. Aretz, I.-K. Jang, K. H. Schlendorf, C. R. Kauffman, M. Shishkov, E. F. Halpern, and B. E. Bouma, "Quantification of macrophage content in atherosclerotic plaques by optical coherence tomography," Circulation, vol. 107, no. 1, pp. 113$119,2003$.

[32] J. M. Poneros, S. Brand, B. E. Bouma, G. J. Tearney, C. C. Compton, and N. S. Nishioka, "Diagnosis of specialized intestinal metaplasia by optical coherence tomography," Gastroenterology, vol. 120, no. 1, pp. 7-12, 2001. 
[33] S. Lam, B. Standish, C. Baldwin, A. McWilliams, A. Gazdar, A. I. Vitkin, V. X. D. Yang, N. Ikeda, C. MacAulay et al., "In vivo optical coherence tomography imaging of preinvasive bronchial lesions," Clinical cancer research, vol. 14, no. 7, pp. 2006-2011, 2008.

[34] A. Fercher, "Ophthalmic interferometer," Optics in Medicine, Biology and Environmental Research, pp. 221-235, 1990.

[35] D. Huang, E. A. Swanson, C. P. Lin, J. S. Schuman, W. G. Stinson, W. Chang, M. R. Hee, T. Flotte, K. Gregory, C. A. Puliafito et al., "Optical coherence tomography," Science, vol. 254, no. 5035, pp. 1178-1181, 1991.

[36] B. Bouma, Handbook of optical coherence tomography. Informa Health Care, 2001.

[37] V. X. D. Yang, M. L. Gordon, A. Mok, Y. Zhao, Z. Chen, R. S. Cobbold, B. C. Wilson, and I. Alex Vitkin, "Improved phase-resolved optical doppler tomography using the kasai velocity estimator and histogram segmentation," Optics Communications, vol. 208, no. 4, pp. 209-214, 2002 .

[38] B. Vakoc, S. Yun, J. De Boer, G. Tearney, and B. Bouma, "Phase-resolved optical frequency domain imaging," Optics Express, vol. 13, no. 14, pp. 5483-5493, 2005.

[39] C. Kasai, K. Namekawa, A. Koyano, and R. Omoto, "Real-time two-dimensional blood flow imaging using an autocorrelation technique," IEEE Trans. Sonics Ultrason, vol. 32, no. 3, pp. 458-464, 1985 .

[40] J. A. Mallery, J. M. Tobis, J. Griffith, J. Gessert, M. McRae, O. Moussabeck, M. Bessen, M. Moriuchi, and W. L. Henry, "Assessment of normal and atherosclerotic arterial wall thickness with an intravascular ultrasound imaging catheter," American Heart Journal, vol. 119, no. 6, pp. 13921400, 1990.

[41] M. Elliott and A. Thrush, "Measurement of resolution in intravascular ultrasound images," Physiological Measurement, vol. 17, no. 4, p. 259, 1996.

[42] M. E. Brezinski, G. J. Tearney, N. Weissman, S. Boppart, B. Bouma, M. Hee, A. Weyman, E. Swanson, J. Southern, and J. Fujimoto, "Assessing atherosclerotic plaque morphology: comparison of optical coherence tomography and high frequency intravascular ultrasound." Heart, vol. 77, no. 5, pp. 397-403, 1997.

[43] A. Maehara, G. S. Mintz, and N. J. Weissman, "Advances in intravascular imaging," Circulation: Cardiovascular Interventions, vol. 2, no. 5, pp. 482-490, 2009.

[44] W. Li, "Image and signal processing in intravascular ultrasound," Ph.D. dissertation, University Medical Center Rotterdam, 1997. 
[45] X. Li, T. H. Ko, and J. G. Fujimoto, "Intraluminal fiber-optic doppler imaging catheter for structural and functional optical coherence tomography," Optics Letters, vol. 26, no. 23, pp. 1906-1908, 2001.

[46] B. A. Standish, K. K. Lee, X. Jin, A. Mariampillai, N. R. Munce, M. F. Wood, B. C. Wilson, I. A. Vitkin, and V. X. D. Yang, "Interstitial doppler optical coherence tomography as a local tumor necrosis predictor in photodynamic therapy of prostatic carcinoma: an in vivo study," Cancer Research, vol. 68, no. 23, pp. 9987-9995, 2008.

[47] V. X. D. Yang, M. Gordon, S.-j. Tang, N. Marcon, G. Gardiner, B. Qi, S. Bisland, E. Seng-Yue, S. Lo, J. Pekar et al., "High speed, wide velocity dynamic range doppler optical coherence tomography (part iii): in vivo endoscopic imaging of blood flow in the rat and human gastrointestinal tracts," Optics Express, vol. 11, no. 19, pp. 2416-2424, 2003.

[48] K. K. C. Lee, A. Mariampillai, J. X. Z. Yu, D. W. Cadotte, B. C. Wilson, B. A. Standish, and V. X. D. Yang, "Real-time speckle variance swept-source optical coherence tomography using a graphics processing unit," Biomedical Optics Express, vol. 3, no. 7, pp. 1557-1564, 2012.

[49] G. J. Tearney, E. Regar, T. Akasaka, T. Adriaenssens, P. Barlis, H. G. Bezerra, B. Bouma, N. Bruining, J.-m. Cho, S. Chowdhary et al., "Consensus standards for acquisition, measurement, and reporting of intravascular optical coherence tomography studiesa report from the international working group for intravascular optical coherence tomography standardization and validation," Journal of the American College of Cardiology, vol. 59, no. 12, pp. 1058-1072, 2012.

[50] G. van Soest, J. G. Bosch, and A. F. Van Der Steen, "Azimuthal registration of image sequences affected by nonuniform rotation distortion," Information Technology in Biomedicine, IEEE Transactions on, vol. 12, no. 3, pp. 348-355, 2008.

[51] A. M. D. Lee, K. Ohtani, C. MacAulay, A. McWilliams, T. Shaipanich, V. X. D. Yang, S. Lam, and P. Lane, "In vivo lung microvasculature visualized in three dimensions using fiber-optic color doppler optical coherence tomography," Journal of Biomedical Optics, vol. 18, no. 5, pp. $050501-$ $050501,2013$.

[52] G. Tearney, M. Brezinski, J. Fujimoto, N. Weissman, S. Boppart, B. Bouma, and J. Southern, "Scanning single-mode fiber optic catheter-endoscope for optical coherence tomography," Optics Letters, vol. 21, no. 7, pp. 543-545, 1996.

[53] D. Bushi, Y. Assaf, Y. Grad, B. Nishri, O. Yodfat, and D. Tanne, "Similarity of the swine vasculature to the human carotid bifurcation: analysis of arterial diameters," Journal of Vascular and Interventional Radiology, vol. 19, no. 2, pp. 245-251, 2008.

[54] D. C. Ghiglia and M. D. Pritt, Two-dimensional phase unwrapping: theory, algorithms, and software. Wiley New York:, 1998. 
[55] B. Braaf, K. A. Vermeer, V. A. D. Sicam, E. van Zeeburg, J. C. van Meurs, and J. F. de Boer, "Phase-stabilized optical frequency domain imaging at 1- $\mu \mathrm{m}$ for the measurement of blood flow in the human choroid," Optics Express, vol. 19, no. 21, pp. 20 886-20 903, 2011.

[56] Y.-J. Hong, S. Makita, F. Jaillon, M. J. Ju, E. J. Min, B. H. Lee, M. Itoh, M. Miura, and Y. Yasuno, "High-penetration swept source doppler optical coherence angiography by fully numerical phase stabilization," Optics Express, vol. 20, no. 3, pp. 2740-2760, 2012.

[57] D. W. Cadotte, A. Mariampillai, A. Cadotte, K. K. Lee, T.-R. Kiehl, B. C. Wilson, M. G. Fehlings, and V. X. D. Yang, "Speckle variance optical coherence tomography of the rodent spinal cord: in vivo feasibility," Biomedical Optics Express, vol. 3, no. 5, pp. 911-919, 2012.

[58] V. X. D. Yang, M. Gordon, E. Seng-Yue, S. Lo, B. Qi, J. Pekar, A. Mok, B. Wilson, and I. Vitkin, "High speed, wide velocity dynamic range doppler optical coherence tomography (part ii): Imaging in vivo cardiac dynamics of xenopus laevis," Optics Express, vol. 11, no. 14, pp. 1650-1658, 2003.

[59] E. W. Beasley and H. R. Ward, "A quantitative analysis of sea clutter decorrelation with frequency agility," Aerospace and Electronic Systems, IEEE Transactions on, no. 3, pp. 468-473, 1968.

[60] P. Karpur and O. J. Canelones, "Split spectrum processing: a new filtering approach for improved signal-to-noise ratio enhancement of ultrasonic signals," Ultrasonics, vol. 30, no. 6, pp. 351-357, 1992.

[61] R. S. Cobbold, Foundations of biomedical ultrasound. Oxford University Press, USA, 2007.

[62] J. A. Jensen, Estimation of blood velocities using ultrasound: a signal processing approach. Cambridge University Press, 1996.

[63] S. Yun, G. Tearney, J. De Boer, and B. Bouma, "Motion artifacts in optical coherence tomography with frequency-domain ranging," Optics Express, vol. 12, no. 13, pp. 2977-2998, 2004.

[64] S. Canada, "Cansim table 102-0529: Deaths, by cause, chapter ix: Diseases of the circulatory system (i00 to i99), age group and sex, canada, annual (number), 2000 to 2006." Released May 4, 2010.

[65] "Tracking heart disease and stroke in canada," Released June 2009.

[66] G. W. Petty, R. D. Brown, J. P. Whisnant, J. D. Sicks, W. M. O'Fallon, and D. O. Wiebers, "Ischemic stroke subtypes a population-based study of incidence and risk factors," Stroke, vol. 30, no. 12 , pp. 2513-2516, 1999.

[67] S. S. Investigators et al., "Stenting of symptomatic atherosclerotic lesions in the vertebral or intracranial arteries (ssylvia) study results," Stroke, vol. 35, no. 6, pp. 1388-1392, 2004. 
[68] E. R. Bates, J. D. Babb, D. E. Casey, C. U. Cates, G. R. Duckwiler, T. E. Feldman, W. A. Gray, K. Ouriel, E. D. Peterson, K. Rosenfield et al., "Accf/scai/svmb/sir/asitn 2007 clinical expert consensus document on carotid stentinga report of the american college of cardiology foundation task force on clinical expert consensus documents (accf/scai/svmb/sir/asitn clinical expert consensus document committee on carotid stenting)," Journal of the American College of Cardiology, vol. 49, no. 1, pp. 126-170, 2007.

[69] C. Setacci and P. De Rango, "A light in the shadows of carotid artery stenting," European Journal of Vascular and Endovascular Surgery, vol. 39, no. 5, pp. 527-528, 2010.

[70] S. Morlacchi, B. Keller, P. Arcangeli, M. Balzan, F. Migliavacca, G. Dubini, J. Gunn, N. Arnold, A. Narracott, D. Evans et al., "Hemodynamics and in-stent restenosis: micro-ct images, histology, and computer simulations," Annals of biomedical engineering, vol. 39, no. 10, pp. 2615-2626, 2011.

[71] A. O. Frank, P. W. Walsh, and J. E. Moore, "Computational fluid dynamics and stent design," Artificial Organs, vol. 26, no. 7, pp. 614-621, 2002.

[72] D. M. Wootton and D. N. Ku, "Fluid mechanics of vascular systems, diseases, and thrombosis," Annual Review of Biomedical Engineering, vol. 1, no. 1, pp. 299-329, 1999.

[73] G. de Donato, F. Setacci, P. Sirignano, G. Galzerano, A. Cappelli, and C. Setacci, "Optical coherence tomography after carotid stenting: Rate of stent malapposition, plaque prolapse and fibrous cap rupture according to stent design," European Journal of Vascular and Endovascular Surgery, vol. 45, no. 6, pp. 579-587, 2013.

[74] K. C. Koskinas, Y. S. Chatzizisis, A. P. Antoniadis, and G. D. Giannoglou, "Role of endothelial shear stress in stent restenosis and thrombosispathophysiologic mechanisms and implications for clinical translation," Journal of the American College of Cardiology, vol. 59, no. 15, pp. 1337-1349, 2012 .

[75] A. Reininger, C. Reininger, U. Heinzmann, and L. Wurzinger, "Residence time in niches of stagnant flow determines fibrin clot formation in an arterial branching model-detailed flow analysis and experimental results." Thrombosis and Haemostasis, vol. 74, no. 3, pp. 916-922, 1995.

[76] K. S. Cunningham and A. I. Gotlieb, "The role of shear stress in the pathogenesis of atherosclerosis," Laboratory Investigation, vol. 85, no. 1, pp. 9-23, 2004.

[77] D. Rockwell, "Vortex-body interactions," Annual Review of Fluid Mechanics, vol. 30, no. 1, pp. 199-229, 1998.

[78] J. E. Moore Jr and J. L. Berry, "Fluid and solid mechanical implications of vascular stenting," Annals of Biomedical Engineering, vol. 30, no. 4, pp. 498-508, 2002.

[79] J. L. Berry, A. Santamarina, J. E. Moore Jr, S. Roychowdhury, and W. D. Routh, "Experimental and computational flow evaluation of coronary stents," Annals of biomedical engineering, vol. 28, no. 4, pp. 386-398, 2000. 
[80] D. Katritsis, A. Theodorakakos, I. Pantos, A. Andriotis, E. Efstathopoulos, G. Siontis, N. Karcanias, S. Redwood, and M. Gavaises, "Vortex formation and recirculation zones in left anterior descending artery stenoses: computational fluid dynamics analysis," Physics in Medicine and Biology, vol. 55, no. 5, p. 1395, 2010.

[81] V. Sheth and A. Ritter, "Using computational fluid dynamics model to predict changes in velocity properties in stented carotid artery," Ph.D. dissertation, Stevens Institute of Technology, 2010.

[82] A. R. Williams, B.-K. Koo, T. J. Gundert, P. J. Fitzgerald, and J. F. LaDisa Jr, "Local hemodynamic changes caused by main branch stent implantation and subsequent virtual side branch balloon angioplasty in a representative coronary bifurcation," Journal of applied physiology, vol. 109, no. 2, pp. 532-540, 2010.

[83] N. Duraiswamy, R. T. Schoephoerster, M. R. Moreno, and J. E. Moore Jr, "Stented artery flow patterns and their effects on the artery wall," Annu. Rev. Fluid Mech., vol. 39, pp. 357-382, 2007.

[84] S. Zhao, X. Xu, A. Hughes, S. Thom, A. Stanton, B. Ariff, and Q. Long, "Blood flow and vessel mechanics in a physiologically realistic model of a human carotid arterial bifurcation," Journal of Biomechanics, vol. 33, no. 8, pp. 975-984, 2000.

[85] O. Greil, G. Pflugbeil, K. Weigand, W. Weiß, D. Liepsch, P. C. Maurer, and H. Berger, "Changes in carotid artery flow velocities after stent implantation: a fluid dynamics study with laser doppler anemometry," Journal of Endovascular Therapy, vol. 10, no. 2, pp. 275-284, 2003.

[86] Q. Long, X. Y. Xu, B. Ariff, S. A. Thom, A. D. Hughes, and A. V. Stanton, "Reconstruction of blood flow patterns in a human carotid bifurcation: a combined cfd and mri study," Journal of Magnetic Resonance Imaging, vol. 11, no. 3, pp. 299-311, 2000.

[87] M. D. Ford, N. Alperin, S. H. Lee, D. W. Holdsworth, and D. A. Steinman, "Characterization of volumetric flow rate waveforms in the normal internal carotid and vertebral arteries," Physiological Measurement, vol. 26, no. 4, p. 477, 2005.

[88] R. Vanninen, H. Manninen, and S. Soimakallio, "Imaging of carotid artery stenosis: clinical efficacy and cost-effectiveness." American journal of neuroradiology, vol. 16, no. 9, pp. 1875-1883, 1995.

[89] G. Nakazawa, S. K. Yazdani, A. V. Finn, M. Vorpahl, F. D. Kolodgie, and R. Virmani, "Pathological findings at bifurcation lesionsthe impact of flow distribution on atherosclerosis and arterial healing after stent implantation," Journal of the American College of Cardiology, vol. 55, no. 16, pp. 1679-1687, 2010.

[90] W. Blackshear, D. Phillips, P. Chikos, J. Harley, B. Thiele, and D. Strandness, "Carotid artery velocity patterns in normal and stenotic vessels." Stroke, vol. 11, no. 1, pp. 67-71, 1980.

[91] D. K. Lopes and A. K. Johnson, "Evaluation of cerebral artery perforators and the pipeline embolization device using optical coherence tomography," Journal of Neurointerventional Surgery, pp. neurintsurg-2011, 2011. 
[92] J. R. Cebral, M. A. Castro, J. E. Burgess, R. S. Pergolizzi, M. J. Sheridan, and C. M. Putman, "Characterization of cerebral aneurysms for assessing risk of rupture by using patient-specific computational hemodynamics models," American Journal of Neuroradiology, vol. 26, no. 10, pp. 2550-2559, 2005.

[93] V. Rayz, L. Boussel, M. Lawton, G. Acevedo-Bolton, L. Ge, W. Young, R. Higashida, and D. Saloner, "Numerical modeling of the flow in intracranial aneurysms: prediction of regions prone to thrombus formation," Annals of Biomedical Engineering, vol. 36, no. 11, pp. 1793-1804, 2008.

[94] R. Torii, M. Oshima, T. Kobayashi, K. Takagi, and T. E. Tezduyar, "Fluid-structure interaction modeling of a patient-specific cerebral aneurysm: influence of structural modeling," Computational Mechanics, vol. 43, no. 1, pp. 151-159, 2008.

[95] D. Fiorella, F. C. Albuquerque, P. Han, and C. G. McDougall, "Preliminary experience using the neuroform stent for the treatment of cerebral aneurysms," Neurosurgery, vol. 54, no. 1, pp. 6-17, 2004.

[96] T. Liou and Y. Li, "Alteration of hemodynamics in a stented lateral aneurysm model by blocking ratios," WSEAS TRANSACTIONS ON FLUID MECHANICS, vol. 1, no. 8, p. 850, 2006.

[97] A. A. Valencia, A. M. Guzmán, E. A. Finol, and C. H. Amon, "Blood flow dynamics in saccular aneurysm models of the basilar artery," Journal of biomechanical engineering, vol. 128, no. 4, pp. $516-526,2006$.

[98] G. Bruno, R. Todor, I. Lewis, and D. Chyatte, "Vascular extracellular matrix remodeling in cerebral aneurysms," Journal of Neurosurgery, vol. 89, no. 3, pp. 431-440, 1998.

[99] J. Frösen, A. Piippo, A. Paetau, M. Kangasniemi, M. Niemelä, J. Hernesniemi, and J. Jääskeläinen, "Remodeling of saccular cerebral artery aneurysm wall is associated with rupture histological analysis of 24 unruptured and 42 ruptured cases," Stroke, vol. 35, no. 10, pp. 2287-2293, 2004.

[100] K. Kataoka, M. Taneda, T. Asai, A. Kinoshita, M. Ito, and R. Kuroda, "Structural fragility and inflammatory response of ruptured cerebral aneurysms a comparative study between ruptured and unruptured cerebral aneurysms," Stroke, vol. 30, no. 7, pp. 1396-1401, 1999.

[101] T. A. Kosierkiewicz, S. M. Factor, and D. W. Dickson, "Immunocytochemical studies of atherosclerotic lesions of cerebral berry aneurysms," Journal of Neuropathology 83 Experimental Neurology, vol. 53, no. 4, pp. 399-406, 1994.

[102] B. Vuong, B. Standish, C. Sun, G. Tearney, R. Kiehl, T. van der Kwast, J. Koo, B. C. Wilson, J. de Morais, L. da Costa et al., "3d optical coherence tomography and digital pathology," in Conference on Lasers and Electro-Optics/Pacific Rim. Optical Society of America, 2011, p. C353. 
[103] B. J. Vakoc, R. M. Lanning, J. A. Tyrrell, T. P. Padera, L. A. Bartlett, T. Stylianopoulos, L. L. Munn, G. J. Tearney, D. Fukumura, R. K. Jain et al., "Three-dimensional microscopy of the tumor microenvironment in vivo using optical frequency domain imaging," Nature medicine, vol. 15, no. 10, pp. 1219-1223, 2009.

[104] H. D. Intengan and E. L. Schiffrin, "Vascular remodeling in hypertension roles of apoptosis, inflammation, and fibrosis," Hypertension, vol. 38, no. 3, pp. 581-587, 2001.

[105] A. Gajjar, R. Hernan, M. Kocak, C. Fuller, Y. Lee, P. J. McKinnon, D. Wallace, C. Lau, M. Chintagumpala, D. M. Ashley et al., "Clinical, histopathologic, and molecular markers of prognosis: toward a new disease risk stratification system for medulloblastoma," Journal of Clinical Oncology, vol. 22, no. 6, pp. 984-993, 2004.

[106] V. Kumar, A. K. Abbas, N. Fausto, and J. C. Aster, Robbins and Cotran Pathologic Basis of Disease, Professional Edition: Expert Consult-Online, 8th ed. Elsevier Health Sciences, 2009.

[107] M. Dennis, B. J. Spiegler, C. R. Hetherington, and M. L. Greenberg, "Neuropsychological sequelae of the treatment of children with medulloblastoma," Journal of Neuro-oncology, vol. 29, no. 1, pp. 91-101, 1996.

[108] T. Gudrunardottir, B. Lannering, M. Remke, M. D. Taylor, E. M. Wells, R. F. Keating, and R. J. Packer, "Treatment developments and the unfolding of the quality of life discussion in childhood medulloblastoma: a review," Child's Nervous System, vol. 1, no. 1, pp. 1-12, 2014.

[109] H. Böhringer, D. Boller, J. Leppert, U. Knopp, E. Lankenau, E. Reusche, G. Hüttmann, and A. Giese, "Time-domain and spectral-domain optical coherence tomography in the analysis of brain tumor tissue," Lasers in Surgery and Medicine, vol. 38, no. 6, pp. 588-597, 2006.

[110] H. Böhringer, E. Lankenau, F. Stellmacher, E. Reusche, G. Hüttmann, and A. Giese, "Imaging of human brain tumor tissue by near-infrared laser coherence tomography," Acta neurochirurgica, vol. 151, no. 5, pp. 507-517, 2009.

[111] S. W. Jeon, M. A. Shure, K. B. Baker, D. Huang, A. M. Rollins, A. Chahlavi, and A. R. Rezai, "A feasibility study of optical coherence tomography for guiding deep brain probes," Journal of Neuroscience Methods, vol. 154, no. 1, pp. 96-101, 2006.

[112] F. J. van der Meer, D. J. Faber, D. B. Sassoon, M. C. Aalders, G. Pasterkamp, and T. G. van Leeuwen, "Localized measurement of optical attenuation coefficients of atherosclerotic plaque constituents by quantitative optical coherence tomography," Medical Imaging, IEEE Transactions on, vol. 24, no. 10, pp. 1369-1376, 2005.

[113] G. van Soest, S. KoljenoviÄ, B. E. Bouma, G. J. Tearney, J. W. Oosterhuis, P. W. Serruys, A. F. van der Steen, N. Gonzalo, S. van Noorden, G. L. van Leenders et al., "Atherosclerotic tissue characterization in vivo by optical coherence tomography attenuation imaging," Journal of Biomedical Optics, vol. 15, no. 1, pp. 011 105-011 105, 2010. 
[114] C. Xu, S. G. Carlier, R. Virmani, and J. M. Schmitt, "Characterization of atherosclerosis plaques by measuring both backscattering and attenuation coefficients in optical coherence tomography," Journal of Biomedical Optics, vol. 13, no. 3, pp. 034 003-034 003, 2008.

[115] G. van Soest, M. Villiger, E. Regar, G. J. Tearney, B. E. Bouma, and A. F. van der Steen, "Frequency domain multiplexing for speckle reduction in optical coherence tomography," Journal of Biomedical Optics, vol. 17, no. 7, pp. 0760181-0760187, 2012.

[116] L. Scolaro, R. A. McLaughlin, B. R. Klyen, B. A. Wood, P. D. Robbins, C. M. Saunders, S. L. Jacques, and D. D. Sampson, "Parametric imaging of the local attenuation coefficient in human axillary lymph nodes assessed using optical coherence tomography," Biomedical Optics Express, vol. 3, no. 2, pp. 366-379, 2012.

[117] B. R. Klyen, L. Scolaro, T. Shavlakadze, M. D. Grounds, and D. D. Sampson, "Optical coherence tomography can assess skeletal muscle tissue from mouse models of muscular dystrophy by parametric imaging of the attenuation coefficient," Biomedical Optics Express, vol. 5, no. 4, pp. 1217-1232, 2014.

[118] P. Gong, R. A. McLaughlin, Y. M. Liew, P. R. Munro, F. M. Wood, and D. D. Sampson, "Assessment of human burn scars with optical coherence tomography by imaging the attenuation coefficient of tissue after vascular masking," Journal of Biomedical Optics, vol. 19, no. 2, pp. 021 111-021 111, 2014.

[119] C. P. Fleming, J. Eckert, E. F. Halpern, J. A. Gardecki, and G. J. Tearney, "Depth resolved detection of lipid using spectroscopic optical coherence tomography," Biomedical Optics Express, vol. 4, no. 8, pp. 1269-1284, 2013.

[120] B. Vuong, C. Sun, M. K. Harduar, A. Mariampillai, K. Isamoto, C. Chong, B. A. Standish, and V. X. D. Yang, "23 khz mems based swept source for optical coherence tomography imaging," in Engineering in Medicine and Biology Society, EMBC, 2011 Annual International Conference of the IEEE. IEEE, 2011, pp. 6134-6137.

[121] R. Rodríguez and A. G. Suarez, "A new algorithm for image segmentation by using iteratively the mean shift filtering," Scientific Research and Essays, vol. 1, no. 2, pp. 043-049, 2006.

[122] K. Barwari, D. M. de Bruin, E. C. Cauberg, D. J. Faber, T. G. van Leeuwen, H. Wijkstra, J. de la Rosette, and M. P. Laguna, "Advanced diagnostics in renal mass using optical coherence tomography: a preliminary report," Journal of Endourology, vol. 25, no. 2, pp. 311-315, 2011. 
REFERENCES 